\title{
GENERATION OF MID-OCEAN EDDIES: THE LOCAL BAROCLINIC INSTABILITY HYPOTHESIS
}

\author{
by \\ Brian Kenneth Arbic \\ B.S., Unıversity of Michigan, 1988 \\ Submitted in partial fulfillment of the \\ requirements for the degree of \\ Doctor of Philosophy \\ at the
MASSACHUSETTS INSTITUTE OF TECHNOLOGY
and the \\ WOODS HOLE OCEANOGRAPHIC INSTITUTION
}

August 2000

(c) Brian K Arbic, 2000

The author hereby grants to MIT and to WHOI permission to reproduce paper and electronic copies of this thesis in whole or in part and to distribute them publicly

Signature of Aldthor

Joint Program in Physical Oceanography Massachusetts Institute of Technology Woods Hole Oceanographic Institution

August 10, 2000

Certified by .....

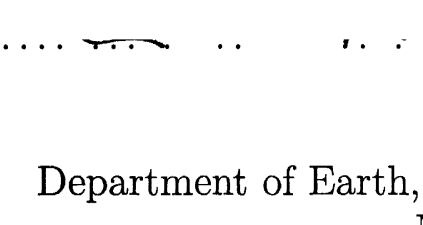

Massachusetts Institute

Glenn R. Flierl Professor

Department of Earth, Atmospheric, and Planetary Sciences Thesis Supervisor

Accepted by W. Brechner Owens Chairman, Joint Committee for Physical Oceanography Massachusetts Institute of Technology Woods Hole Oceanographic Instıtution 


\title{
GENERATION OF MID-OCEAN EDDIES: THE LOCAL BAROCLINIC INSTABILITY HYPOTHESIS
}

by

\author{
Brian Kenneth Arbic
}

\begin{abstract}
Submitted in partial fulfillment of the requirements for the degree of Doctor of Philosophy at the Massachusetts Institute of Technology and the Woods Hole Oceanographic Institution
\end{abstract}

\begin{abstract}
The plausibility of local baroclinic instability as a generation mechanism for midocean mesoscale eddies is examined with a two-layer, quasi-geostrophic (QG) model forced by an imposed, horizontally homogeneous, vertically sheared mean flow and dissipated through bottom Ekman friction. Explanations are sought for two observed features of mid-ocean eddies: 1) substantial energy is retained in the baroclinic mode and in the associated deformation radius $\left(R_{d}\right)$ scale, and 2) the ratio of eddy to mean kinetic energy is much larger than one.

The tendency of QG to cascade energy into the barotropic mode and into scales larger than $R_{d}$ can be counteracted when stratification is surface-trapped, for then the baroclinic mode is weakly damped, and hence enhanced. Numerical experiments are performed with both surface-trapped and uniform stratification to quantify this. Experiments with equal Ekman frictions in the two layers are also performed for purposes of contrast. Interpretation is aided with an inequality derived from the energy and enstrophy equations. The inequality forbids the simultaneous retention. of substantial energy in the baroclinic mode and in scales near $R_{d}$ when Ekman friction is symmetric, but points towards surface-trapped stratification and bottomtrapped friction as an environment in which both of these can be achieved.

The dissertation also contains a systematic study of geostrophic turbulence forced by nonzonal flows. Narrow zonal jets emerge when shear-induced mean potential vorticity (PV) gradients are small compared to the planetary gradient $(\beta)$, and energy is a strong function of the angle shear presents to the east-west direction. When shear-induced PV gradients are comparable to $\beta$, and the mean shear has a westward component, fields of monopolar vortices form and persist. Energy is asymmetric between fields of cyclones and anticyclones. Such asymmetry was commonly thought not to occur in $\mathrm{QG}$, but is shown here to be introduced by the nonzonal basic state. In both jet and vortex regimes, eddy energy can be much larger than mean kinetic energy, contrary to the expectation that $\beta$ stabilizes weak shear flows.
\end{abstract}


Thesis Supervisor: Glenn R. Flierl

Title: Professor

Department of Earth, Atmospheric, and Planetary Sciences

Massachusetts Institute of Technology 


\section{Acknowledgments}

Six years in graduate school generates a lot of debt, fortunately not too much of it financial in nature. Consequently, these acknowledgements, like the rest of my thesis, will not be short. My apologies in advance to those whom I may have inadvertently left out.

First and foremost, I would like to thank my advisor, Glenn Flierl. Glenn is extremely smart, and well-versed in a wide variety of topics. What other advisor could advise a student on SCSI versus IDE as adeptly as on cascades in forceddissipated turbulence? He is known in the community for being amazingly generous with his time. I've benefitted from countless fruitful discussions with him over the past four years. Perhaps most importantly, he has extraordinary patience for those of us who need to go over things several times before we understand them.

I thank Breck Owens for giving me a very good research project during my first summer in the program. I might have actually been able to finish it then had I been adept with computers. As this was not the case, it became my generals project, and then a side project I worked on alongside this thesis-at times a difficult balancing act. Minutes before my thesis defense, I found out that it had been accepted by the Journal of Climate. Because the project was so different in nature from my thesis, it contributed a lot to my education as an oceanographer. I thank Breck as well for serving on my thesis committee, and improving this document with his comments.

I also thank my other thesis committee members, for their many helpful suggestions. Like everyone else in EAPS who had Alan Plumb on their thesis committee, I was glad I made that choice. He never complained about the oceanographic applications of my GFD thesis, nor about having to spend time at WHOI on my account. Nelson Hogg generally made the summary statement at the end of committee meetings, for better or worse, which was useful. He pushed me the hardest to relate the thesis work to actual oceanic observations, an important thing for a numerical modeler to hear. Mike Spall did some of the work which inspired me to look at 
nonzonal flows, and was always available for discussions in his office. Isaac Held served from afar, and did manage to attend the thesis defense, which was greatly appreciated. In the meantime, I managed to meet him here and there at meetings, and his comments were always on the mark. I thank Sonya Legg for chairing my thesis defense, which she ran very smoothly. I also learned a lot from sitting in on her turbulence class during my final semester here.

At one point or another I've probably talked to all of the other Joint Program faculty as well, and I thank all of them for providing a stimulating atmosphere in which to conduct research on the ocean. I'd like to thank John Marshall for encouraging me to enroll in the Joint Program, and for many illuminating discussions over the years. Amy Bower helped orient me to the field of oceanography during my first summer. Karl Helfrich, Jim Price, Jochem Marotzke, Reginald Newell, Carl Wunsch, Peter Stone, Rick Rosen, Lodovica Illari, and Joe Pedlosky all offered excellent courses during my first two years here. Larry Pratt and Mike Spall did as well, and gave me a $\mathrm{C}$ when I deserved it, which helped build a little character. Carl Wunsch, Paola Rizzoli, Jochem Marotzke, Joe Pedlosky, Kurt Polzin, Ray Schmitt, Terry Joyce, Mike McCartney, Mindy Hall, Phil Richardson, Rui Xin Huang, Dave Chapman, Bruce Warren, and Joe LaCasce have all contributed useful advice. Jim Price and Ken Brink gave helpful advice during times I wasn't so happy-for instance, after my oral exam, which was a nightmare. I've also gotten encouragement from faculty I've met from other institutions-Arnold Gordon, Bill Smethie, Lorenzo Polvani, Mark Cane, Rick Salmon, Paola Cessi, Harry Bryden, and Goeff Vallis come to mind, for instance.

At one point near the end of my thesis research, I was performing model runs on forty processors at once. Many people generously assisted me during this phase of the work. John Marshall and Chris Hill allowed me access to an embarassingly large number of machines. Helen Hill, Arnaud Czaja, Parvadha Suntharalingam, Stephanie Dutkiewicz, Daniel Jamous, Paul van der Vaart, and Richard Karsten all 
kindly allowed me to run in background on their desktops. Francesco Paparella and Janet Fields let me run on the GFD machines during the off-season of the program. Mike Spall let me use his SGI, while Nelson Hogg and Breck Owens let me use their new Hewlett-Packards; Jim Price and Eric Cunningham helped me with these as well. John Farrington and Stella Callagee procured a computer so that I could have my own disk to put all the other results on. Thanks to Geoff Vallis for pointing me towards the "Fastest Fourier Transform in the West" which doubled the speed of Glenn's model. Otherwise I would have needed eighty processors.

The technical staff at MIT has been very helpful over the years. I'd like to thank Scott Blomquist, Linda Meinke, Charmaine King, Diana Spiegel, and Bud Brown for help in various aspects of computing and preparation of figures. There are many great people on the WHOI technical staff as well, including Jane Hopewood, Bob Heimerdinger, Chris Wooding, and Marg Zemanovic, my irrepressible WHOI officemate. Others, whom I've had the pleasure of working with at sea, are Shelley Ugstad, Marshall Schwartz, Terry McKee, Dan Torres, Dave Wellwood, Frank Bahr, and Jerry Dean. It was an honor to stand CTD watch with Bob Millard, who did so much to calibrate it in the early years.

Bob Pickart took me to sea twice, a terrific experience which I will always cherish. It's a miracle he did take me after hearing about my miserable first cruise, most of which I spent vomiting into a blue bucket. The crew of both the Knorr and Endeavor were terrific, and the food was free, plentiful, and tasty, although I had to go out on the second and third cruises to discover that.

The Joint Program functions so well because of the many capable people at both WHOI and MIT who put in the effort to keep it running smoothly. Julia Westwater, Stella Callagee, Jake Pierson, John Farrington, Marcey Simon, and Stacey Drange have all been very helpful from the WHOI Education Office side of things. I especially appreciate the housing opportunities we have in Woods Hole through their efforts. It has been a pleasure to spend several summers on the Cape, and a day here 
and there at a moments' notice. Ronni Schwartz holds down the Joint Program fort ably at MIT, and has become a close friend. Much of what I know about classical music was learned from her. She's probably attended my annual talk on my Peace Corps/Africa experience more times than anyone else, and she also introduced me to Elaine.

Unfortunately the rigors of graduate schooldom have led me to lose touch with many of my pre-graduate school friends. I've managed to stay in touch with some of them, however, such as Jim Bender, Jeff Stehr, Joel Krupa and Jim Gerrish, which has occasionally forced me to ponder the reality that most of the world does not revolve around academic research. The Boston Area Returned Peace Corps Association has also been useful in that endeavor. Thanks to Burt and Jane deFrees for several wonderful weekends in Maine. Intramural ice-hockey and softball, numerous ethnic restaurants in Boston and Cambridge, and the mountains of northern New England have also been welcome distractions, as have trips to Alaska, Venezuela, southeast Asia, the southern Appalachians, canyon country in Utah, and the Sierra Nevadas.

I've developed many friends among fellow students and postdocs. Mike Atkins and Bill Shaw were housemates my first summer, and have remained friends. Tom Marchitto was a fine housemate my second year, and Dave Amundsen was a good housemate and friend during our four bachelor years at Frank's place. My officemates during my first few years here, Francois Primeau, Lou St. Laurent, and Albert Fischer, have been close friends and colleagues. Albert, being Swiss, stayed neutral during discussions the rest of us had which were loud, opinionated, and not altogether wise. Albert and Lou were also classmates of mine, as were Steve Jayne and Stephanie Harrington. Lou beat me to the PhD by a year, Albert by two weeks. Steve took the generals early and finished even earlier then Lou. Constantine Giannitsis, Jeff Scott, and Vince Larson, who entered the same year but in meteorology, have been good friends. Jeff and Constantine were kind enough to defend after me 
so that I wouldn't be the last one. Once Francois graduated I had hopes of being the best player on the EAPS hockey team, but along came Richard Karsten and it was not to be. Richard Wardle and I spent many a lunch stuffing ourselves with Indian food. Juli Atherton and Yu-Han Chen put up with me during the final year of thesis writing. Juli still asks me to join her for lunch occasionally, so I guess I haven't been too horrible. Chris Edwards, Jim Gunson, Paul Robbins, Ed Dever, Sarah Gille, Alison McDonald, Keith Alverson, Jay Austin, Derek Fong, Miles Sundermeyer, and Ari Epstein provided the much-needed older students' perspective when I first arrived. It's been fun to get to know others as well, such as Pablo Zurita, Adam Sobel, Jubao Zhang, Alex Ganachaud, Xiaoyun Zang, Judith Wells, Juan Botella, Galen McKinley, Jeff Gebbie, Baylor Fox-Kemper, and Alison Walker. Zan Stine, Markus Jochum, and Payal Parekh have put up well with the grouchy old man in the office. Chris Hill has spent a lot of time helping me out and shooting the breeze. He pretends to be hard-hearted but those of who know him better know better. Tom Haine taught me many important reasons that the U.K. is superior to the U.S., but still came back to the U.S. to live and work. Mick Follows is a super person, who, among other favors, sat through one of my practice talks. Arnaud Czaja did likewise, and has one of the best senses of humor around. Paul van der Vaart has patiently endured many hours of me rambling about my vortices. Gidon Eshel is a great guy and I was sorry to see him leave the area. Samar Khatiwala, Richard Karsten, Daniel Jamous, Patrick Heimbach, Francis Condi, Nadia Ayoub, Stephanie Dutkiewicz, and Helen Hill have also contributed to the fun I've had here.

The best thing that happened to me during my time here has been meeting Elaine Chew. For some reason she has stuck with me. Through her I've met whole new sets of friends and family, too numerous to list. Her enthusiasm for things is as infectious as her smile. Her determination to make time for the things she loves, such as music, is inspiring. Her concerts and thesis work, from the point of view of this unbiased observer, are seminal. She has been very supportive during the last, 
difficult period of thesis-writing, driving up from Pennsylvania to attend my thesis defense. My parents love her dearly, as do I, and who can blame us.

It's been great living near Vermont, where my mother's side is from. I've taken many a trip up there to enjoy the Green Mountains and visit with Grandma Bartlett, Uncle Rick, and Aunt Bonnie. I've met up with my brother Joel, who lives in Pheonix, three times during my student tenure, for trips to various mountains and canyons. My other brother Dan has provided me with a niece, whom we all agree is extraordinarily gifted. Grandma Arbic is still a source of wisdom, though she doesn't understand my predilection for mountains.

Saving the most important for last, my parents are thanked for their support and love through the years, through countless trials and tribulations, and for always believing in the things I do, even when others shake their heads.

My first three years here were funded by an Office of Naval Research/National Defense Science and Engineering Graduate Fellowship administered by Jeff Jarocz at the American Society for Engineering Education. The fellowship included extra funds with which I bought a computer. Many of the initial results for this dissertation were obtained on this computer. During the last three years, most of my support has come the National Science Foundation via grant OCE-9617848, with some additional support coming from the Office of Naval Research via grant N00014-95-1-0824. 


\section{Contents}

$\begin{array}{ll}\text { Abstract } & 3\end{array}$

$\begin{array}{ll}\text { Acknowledgments } & 5\end{array}$

1 Introduction $\quad 15$

1.1 Previous work on local and nonlocal generation . . . . . . . . . 17

1.2 Homogeneous turbulence as a model of the ocean interior . . . . . . . 22

1.3 Previous work in homogeneous geostrophic turbulence . . . . . . . . . 24

1.4 Contributions of this dissertation . . . . . . . . . . . 26

1.4.1 Effects of stratification and friction on vertical structure of eddies ........................... 27

1.4.2 Effects of stratification and friction on horizontal scales of eddies 27

1.4.3 Generation of strong eddy fields from nonzonal mean flows on a beta plane . . . . . . . . . . . . . . . . 28

1.5 Overview of dissertation . . . . . . . . . . . . . . 29

2 The model $\quad 32$

2.1 Quasi-geostrophic PV equations ................. 32

2.1.1 Modal PV equations ................. 35

2.2 Energy and enstrophy equations . . . . . . . . . . . . . 37

2.2.1 Layer energy and enstrophy equations . . . . . . . . . . 37

2.2.2 Modal energy and enstrophy equations . . . . . . . . . . . . 41 
2.3 Model parameters . . . . . . . . . . . . . . . . . . . . 43

2.4 Numerical method . . . . . . . . . . . . . . . . . . . . . 44

2.5 Small-scale dissipation $\ldots \ldots \ldots \ldots \ldots \ldots$

2.5.1 Large symmetric friction case: no small-scale dissipation needed 47

2.5.2 Implementation of wavenumber cut-off filter . . . . . . . 47

2.5.3 Implementation of hyperviscosity $\ldots \ldots \ldots . \ldots . \ldots 50$

2.6 Linear stability analysis $\ldots \ldots \ldots \ldots \ldots \ldots$

3 Effects of stratification and friction on eddy baroclinicity and length scales 56

3.1 Inverse cascades in two-dimensional flows . . . . . . . . . . . 56

3.2 Barotropization and inverse cascades in stratified QG flows . . . . . 60

3.3 Can the level of barotropization be reduced with surface-trapped stratification and bottom-trapped friction? . . . . . . . . . 64

3.4 Effects of stratification on freely-evolving experiments . . . . . . 67

3.5 Baroclinicity in bottom-friction only forced-dissipated experiments . . 70

3.6 Baroclinicity in forced-dissipated experiments with symmetric friction 90

3.7 A word about friction in the ocean . . . . . . . . . . . 95

3.8 Appearance and length scales of model eddy fields . . . . . . . . . . 96

3.9 Chapter summary . . . . . . . . . . . . . . . . . . . . . 114

4 Quantification of the inverse cascade to large scales and the barotropic $\begin{array}{ll}\text { mode with a cascade inequality } & 121\end{array}$

4.1 Derivation of one-layer cascade inequality . . . . . . . . . . . 122

4.2 Derivation of two-layer cascade inequality . . . . . . . . . . . . 124

4.3 Evaluation of two-layer cascade inequality in symmetric friction case . 126

4.4 Evaluation of two-layer cascade inequality in bottom friction only case 130

4.5 Effects of topography on the cascade inequality . . . . . . . 135

4.6 Chapter summary . . . . . . . . . . . . . . . . . . . . 136 
5 Generation of strong eddy fields from nonzonal mean flows on a beta plane

5.1 Satisfying $\mathrm{J}(\bar{\psi}, \bar{q})=0$ for homogeneous nonzonal flows . . . . . . . 141

5.2 Eddy generation from weak nonzonal flows on a beta plane: effect of

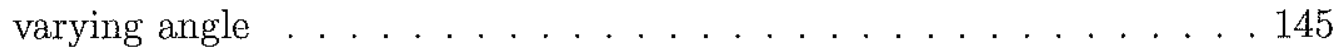

5.3 Effect of varying beta on isotropy of eddies generated from nonzonal mean flows . . . . . . . . . . . . . . . . . . . 168

5.4 Chapter summary . . . . . . . . . . . . . . . . 196

6 Cyclone-anticyclone asymmetry in quasi-geostrophic turbulence forced $\begin{array}{ll}\text { by nonzonal mean flows } & 198\end{array}$

6.1 Sensitivity of vortex solutions to small-scale dissipation . . . . . . . 199

6.2 Dependence of vortex solutions on other parameters . . . . . . . . . 201

6.3 Cyclone-anticyclone asymmetry and dependence on initial conditions 216

6.4 Spin-down and freely decaying experiments initialized by vortex so-

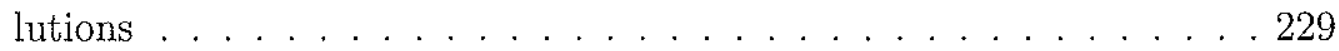

6.4.1 Freely evolving equivalent barotropic turbulence . . . . . . . . 229

6.4.2 Freely decaying equivalent barotropic turbulence initialized by vortex solutions . . . . . . . . . . . . . . . . 232

6.4.3 Two-layer freely decaying turbulence initialized by vortex so-

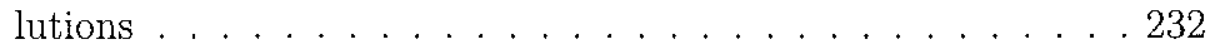

6.4.4 Two-layer turbulence initialized by vortex solutions and dissipated by bottom Ekman friction . . . . . . . . . . . . 238

6.5 Dominant balance of the vortex solutions . . . . . . . . . . . . . . 242

6.6 Comparison to the coherent vortices of earlier studies . . . . . . . 258

6.7 Chapter summary . . . . . . . . . . . . . . . . . 262

7 Conclusions $\quad 267$

7.1 The central question and approach of the dissertation . . . . . . 267 
7.2 Major results of the dissertation . . . . . . . . . . . 269

7.3 New regimes discovered in the dissertation . . . . . . . . . . 272

7.4 Rescaling the equations for different values of friction, shear, and beta 273

7.5 Limitations of homogeneous turbulence models as a representation of the mid-ocean . . . . . . . . . . . . . . . . . . 275

7.5.1 Eddy feedbacks onto the mean . . . . . . . . . . . . 276

7.5 .2 Finite domain size . . . . . . . . . . . . . . 277

7.5.3 Doubly periodic boundary conditions . . . . . . . . . . . 278

7.5 .4 Spinup time . . . . . . . . . . . . . . . . . 278

7.6 Plausibility of the local baroclinic instability mechanism . . . . . 279

7.7 Future work . . . . . . . . . . . . . . . . . . . . . . . . . 279

$\begin{array}{ll}\text { References } & 284\end{array}$ 


\section{Chapter 1}

\section{Introduction}

The energy of the ocean circulation is distributed over a tremendous range of length scales, from planetary and basin scales of thousands of kilometers down to dissipation scales of millimeters. It has been known for some time that the energies of geostrophic currents-that is, currents having length scales comparable to or larger than the deformation radius and time scales longer than a few days-are dominated by features known as mesoscale, or geostrophic, eddies (c.f. Gill et al. 1974 and references therein). Figure 1-1, produced from satellite altimetry data, makes clear three aspects of oceanic flows. First, mean flows are strongly inhomogeneous; most of the mean flow kinetic energy is concentrated in narrow intense currents such as the Gulf Stream, Agulhas, Kuroshio, and Antarctic Circumpolar Current. Second, eddy kinetic energies are also spatially inhomogeneous, decreasing by an order of magnitude as one moves from boundary current regions into the interior. Third, the ratio of eddy to mean kinetic energy is large everywhere. (Note these characteristics of ocean circulation have been known for some time-c.f. Schmitz 1996 and references therein for a summary of early work. Altimetry data provides more comprehensive coverage than earlier methods.)

Because the mesoscale eddy field is vigorous and spatially inhomogeneous, divergences of eddy potential vorticity (PV) fluxes may be large enough to have significant 

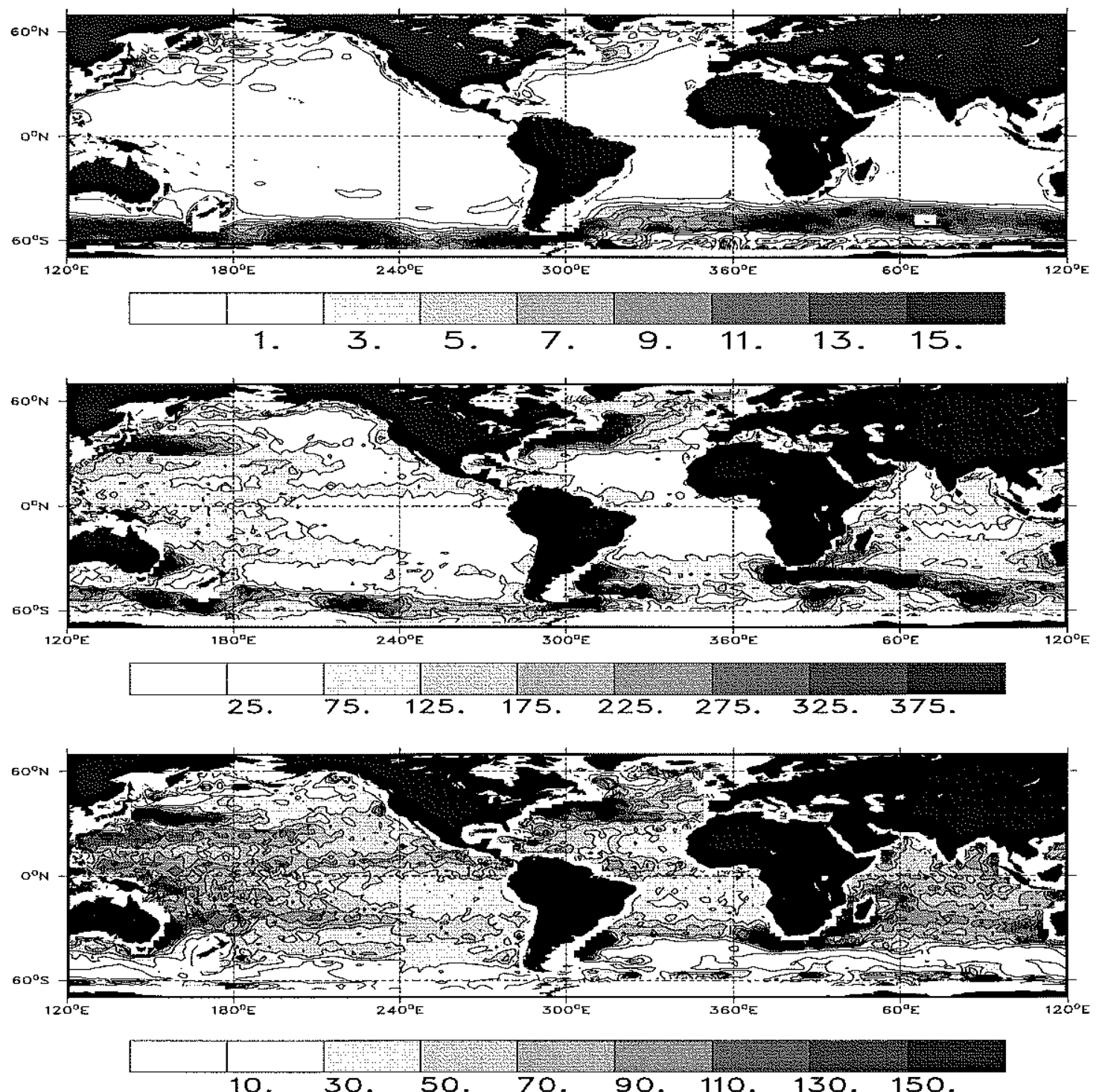

Figure 1-1: Top panel: Global map, produced from altimetric data, of surface kinetic energy of the mean flow multiplied by $\sin ^{2}$ (latitude). Units are $\mathrm{cm}^{2} \mathrm{~s}^{-2}$. Note that a best estimate of the geoid was made to estimate this quantity. Middle panel: Surface eddy kinetic energy multiplied by the same factor, and displayed in the same units. Note that here eddies are defined as deviations from a time mean and therefore encompass phenomena other than mesoscale eddies. Bottom panel: Ratio of eddy to mean kinetic energy at the surface. (Courtesy of Carl Wunsch and Charmaine King, personal communication). 
effects on the large-scale mean ocean circulation, and by extension, the climate of the earth. This is still a matter of current research (c.f. Hogg 1993; Wunsch 1999). At the very least it is clear that eddies are a major source of particle dispersal, and therefore important to biological systems (in which the tracers of interest are nutrients). There has been much interest over the years in parameterizing eddy fluxes of heat, salt, potential vorticity, and passive tracers (c.f. Green 1970; Stone 1972; Gent and McWilliams 1990; Larichev and Held 1995; Wardle and Marshall 1999). Many parameterization schemes are based on the paradigm that eddies are generated via local baroclinic instability. Yet it is not clear that local processes dominate the production of mid-ocean eddies. Strong boundary currents such as the Gulf Stream, Kuroshio, and Agulhas are known to produce intense vortical rings (c.f. Fuglister and Worthington 1951; Cheney and Richardson 1976; Richardson 1983). The author is not aware of any similarly unambiguous evidence of eddy formation in the weak mean flows of the ocean interior. As will be seen shortly, however, there is a long history of plausible arguments that the mid-ocean does produce eddies via local baroclinic instability. Section 1.1 reviews a number of the arguments for local and nonlocal generation of mid-ocean eddies. In section 1.2 a statement of the philosophy behind the use of horizontally homogeneous models in this dissertation will be made, and in section 1.3 a brief history of results obtained from homogeneous models is presented. Section 1.4 outlines the contributions to be made in this dissertation. This introduction concludes with a short overview of succeeding chapters.

\subsection{Previous work on local and nonlocal genera- tion}

The fact that the eddy field is most energetic in regions of strong mean flows has led many to study the possibility that mesoscale eddies are chiefly generated in 
intense currents and then radiated into the ocean interior. Flierl and Kamenkovich (1975) modeled the Gulf Stream as a corrugated wall propagating zonally through an otherwise quiescent ocean. They found that Rossby waves generated by the wall motion remained trapped in the vicinity of the wall, suggesting that it was difficult to radiate energy into the ocean interior. Two possibilities to circumvent this difficulty have been suggested. Hogg (1988) and Malanotte-Rizzoli et al. (1994) found that a zonal current which meanders stochastically can radiate disturbances into the interior, and Kamenkovich and Pedlosky (1996) found that nonzonal jets can radiate into the interior. Observational evidence for such radiation was given in Hogg (1994), as well as Chester et al. (1994). The latter study employed tomographic data to calculate the wave activity flux vector $\mathbf{M}_{R}$ for time-mean flows, first developed by Plumb (1986). Chester et al. showed that $\mathbf{M}_{R}$ was directed away from the Gulf Stream, indicating that it is a source of eddy activity for nearby regions. There is another possibility for Gulf Stream eddy energy to penetrate into the ocean interior: instead of energy propagating in the form of waves, it may propagate in the form of rings. Flierl (1977) produced maps of the probability of finding a ring in a particular part of the North Atlantic, and multiplied this probability by the average energy of a ring. The resulting map is qualitatively similar to maps of the mid-ocean eddy energy, suggesting that rings and the waves they generate may be responsible for much of the mid-ocean eddy field.

Direct observational evidence of baroclinic instability in the mid-ocean has proven difficult to find, but there have been many attempts to find indirect signatures of it. For example, Bryden (1979) and Bryden (1982) found evidence of eddy heat fluxes directed down mean temperature gradients, consistent with local baroclinic instability, in the Antarctic Circumpolar Current and Gulf Stream recirculation regions, respectively. Hogg (1985) also argued that baroclinic instability is active in the recirculation region. He found that zonal phase propagation as a function of frequency, horizontal scales at the peaks in the time spectrum, and vertical phase 
shifts, were qualitatively similar to predictions from linear baroclinic instability theory. However, Fu et al. (1982) found no convincing evidence of the energy conversions predicted by instability theory in the Atlantic North Equatorial Current. These studies all utilized current meters. Stammer (1997) displayed maps of available potential energy of the mean circulation (computed from the Levitus (1994) climatology) next to maps of eddy kinetic energy derived from altimetry data, and noted striking qualitative similarities over much of the global ocean. He also showed that eddy velocities are well correlated with mean thermal wind velocities. Finally, he showed that global averages of eddy length scales are well correlated with the first baroclinic mode deformation radius over a wide range of latitudes (figure 1-2). This is consistent with the findings of Mercier and de Verdiere (1985) in eastern North Atlantic current meter records. Since the pioneering efforts of Charney (1947) and Eady (1949) it has been known that the dominant length scales emergent from a linear baroclinic instability analysis are those near the first mode deformation radius. Thus the length scales of mid-ocean eddies are consistent with predictions from linear baroclinic instability theory. (Note, however, that this cannot be taken as proof that the instability mechanism is taking place, since other processes, such as geostrophic adjustment, also take place at this scale-c.f. Cushman-Roisin (1994) and references therein).

Frankignoul and Muller (1979) and Muller and Frankignoul (1981) proposed that ocean eddies are directly generated by rapidly fluctuating wind fields. Their analysis was linear, but has been extended into the nonlinear domain by others, for instance Treguier and Hua (1987) and Treguier and Hua (1988). The latter two studies found that eddy energies comparable to those seen in the eastern (quieter) parts of the Atlantic could be generated from realistic wind forcing levels. However, the more recent study of Large et al. (1991) was unable to generate observed levels of eddy energies in a flat-bottom, quasi-geostrophic model of the North Pacific forced by realistic winds. Stammer and Wunsch (1999) found that significant correlations 

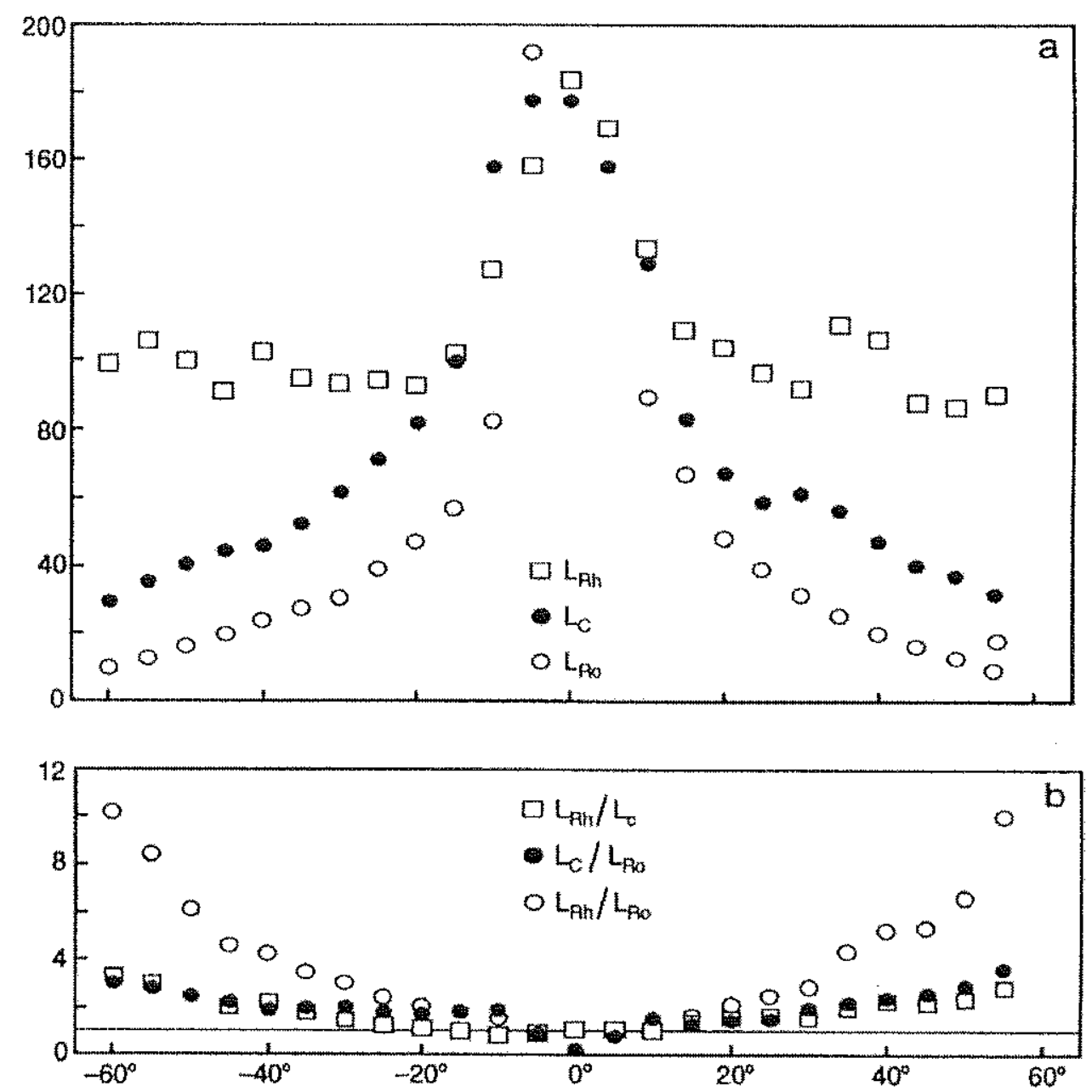

Figure 1-2: Top panel: Length scales in $\mathrm{km}$ as a function of latitude. The deformation radius $L_{R o}$ was calculated from the Levitus (1994) climatology. The Rhines scale $L_{R h}=\sqrt{\frac{U_{e d d y}}{\beta}}$ was calculated from altimetric esimates of surface eddy velocities. $L_{C}=\frac{\lambda}{2 \pi}$, where $\lambda$ is the wavelength of maximum surface kinetic energy in altimetric data. Lower panel: Ratios of the length scales. Figure taken from Stammer (1997). 
between observed variations in eddy kinetic energy and in wind stress were confined to the high latitude regions of the North Atlantic and North Pacific, and even there, only some fraction of the eddy energy could be attributed to direct generation by winds. Their conclusion is that direct eddy generation by the wind probably occurs in the ocean, but that local baroclinic instability is more likely to be a major source of the mid-ocean eddy field.

Two early papers which argued that baroclinic instability of mid-ocean currents is an important source of mid-ocean eddy energy were Gill et al. (1974) and Robinson and McWilliams (1974). Gill et al. (1974) pointed out that vast stores of available potential energy exist in the mid-ocean. They argued via scaling that the ratio of eddy to mean kinetic energy is bounded above by $\left(\frac{L_{b a s i n}}{R_{d}}\right)^{2}$, of order 1000 . Thus the large scale circulation stores more than enough energy to produce eddies as vigorous as those observed. They then demonstrated via a linear stability analysis that some mid-ocean currents are baroclinically unstable. The linear analysis, however, is valid only for small amplitudes, and is therefore unable to demonstrate that the instability process is able to tap into a large fraction of the available potential energy and ultimately produce eddy velocities which are much larger than the mean. This point was discussed in Pedlosky (1975a), who disagreed with Gill et al. (1974) about the likelihood of mid-ocean baroclinic instability as a source of mid-ocean eddy production. He based his argument on a previous weakly nonlinear analysis of eddies produced by a zonal flow in a channel (Pedlosky 1970), in which eddy velocities are found to be bounded by the mean velocities.

Robinson and McWilliams (1974) also performed linear stability analyses on idealized flows with typical mid-ocean parameters. They discussed many factors which affect the instability process, including the nonzonal orientation typical in gyre flows. As discussed in Pedlosky (1987), nonzonal flows are much more difficult to stabilize than are zonal flows. Rhines (1977) noted that large-scale, unforced baroclinic Rossby waves constituted meridional flows on a beta plane and could generate an 
eddy field. Spall (1994) found low-frequency zonal motions in a spin-down calculation of nonzonal flows, and suggested these as an explanation for the low-frequency zonal motions found in the eastern North Atlantic current meter records of Muller and Siedler (1992). Spall (2000) demonstrated via extensions of the scaling arguments of Held and Larichev (1996) that weak nonzonal flows on a beta plane, unlike weak zonal flows on a beta plane, are capable of producing eddy velocities much larger than the weak mean shear. He successfully tested the scaling arguments in a numerical model of a Sverdrup flow driven by an idealized wind-stress curl. Dubus (1999) also examined the equilibration of an eddy field forced by an inhomogeneous meridional flow on a beta plane, and concluded that equilibration involved Rossby wave propagation, dissipation, and baroclinic instability.

One other possibility should be mentioned, although we will not examine it in the body of the dissertation. It may be that most mid-ocean eddies are initially generated nonlocally, for instance in energetic currents such as the Gulf Stream, but that the characteristics of the eddy field (i.e. the vertical structure and horizontal scales) are locally determined by factors such as stratification, bottom topography, etc. This hypothesis was tested by Owens and Bretherton (1978), who simulated the evolution of a specific eddy field in a specific region-the "MODE" region of the North Atlantic-and compared the model output to observations. Their hypothesis, that eddy structures are locally determined, was consistent with observations to within experimental error. As a result, in the real ocean it may be difficult to separate the problem of local versus nonlocal influence on mid-ocean eddy fields.

\subsection{Homogeneous turbulence as a model of the ocean interior}

Mid-ocean mean flows typically vary over length scales of several hundred kilometers or more. Eddy length scales are smaller, typically of order one hundred kilometers 
(Stammer 1997). Therefore a reasonable first approximation of the ocean interior might be a model in which the mean flow doesn't vary at all in the horizontal. A homogeneous model could represent a patch of ocean, large enough to contain several eddy lengths, but small enough for the mean flows to be considered horizontally homogeneous. By definition, homogeneous models cannot have rigid horizontal boundaries. Numerical models of homogeneous turbulence must have finite extent, but true boundaries, which would block motion, can be avoided with doubly periodic boundary conditions. That is, what leaves one side of the model domain enters the other side. There are two major advantages of homogeneous turbulence models for the problem considered in this dissertation. In horizontally homogeneous flows, every point generates the same amount of eddy activity as every other point. Thus there is no ambiguity in sorting out the source of the eddies; it can only be local baroclinic instability. The second advantage is that integrals over the model domain of the energy and enstrophy equations are simpler for homogeneous flows. Some terms in the equations will integrate to zero under doubly periodic conditions, which would not be the case in the presence of real boundaries. This makes interpretation simpler. Actual mid-ocean mean flows, of course, are not homogeneous (figure 1-1). In inhomogeneous flows there will be divergences of eddy fluxes, which then alter the mean flow. This mechanism is absent in the present study. We will return to this limitation of homogeneous models in the conclusions.

Homogeneous turbulence models have traditionally been run in two different limits. One is that of freely evolving, or freely decaying, turbulence. In this case, there is no forcing and no dissipation, other than hyperviscosity or filters used to eliminate structure on scales too small to be resolved numerically. Thus one studies the free evolution of an initial condition. This might be the appropriate limit to study, for example, the propagation of a Gulf Stream ring through a quiet, inviscid interior. Much has been learned about the action of the nonlinear terms in the equations of motion through studies of freely evolving homogeneous turbulence, for instance, the 
tendency of two-dimensional turbulence to form coherent vortices (c.f. McWilliams 1984). However, energy in the ocean is continually being supplied by a variety of sources, and dissipated in bottom boundary layers and through internal wave formation and breaking. Thus, forced-dissipated models more closely reflect true oceanic conditions. Homogeneous turbulence models have been forced in a variety of different ways, for instance, by an imposed horizontal temperature gradient which in turn sets up a baroclinically unstable thermal wind shear (Haidvogel and Held 1980), or by stochastic winds (Treguier and Hua 1987; 1988). For the problem of the midocean gyres, this author will follow the example set by Hua and Haidvogel (1986) and Larichev and Held (1995), in which a baroclinically unstable mean shear flow is imposed between the layers. It is assumed that this mean velocity is maintained by winds blowing over the ocean, but those winds never appear explicitly in the model. Although most of the results in this thesis will be obtained from forced-dissipated model runs, some freely evolving runs will be done to illustrate tendencies of the governing equations.

\subsection{Previous work in homogeneous geostrophic turbulence}

On large scales, geophysical flows tend to be nearly two dimensional because of the stiffening influence of rotation. Because of its two-dimensional nature, turbulence in large-scale geophysical flows is profoundly different from turbulence in threedimensional flows. In three-dimensional turbulence, nonlinear interactions effect a cascade of energy from large scales down to the small scales where dissipation takes place (c.f. Kundu 1990 for a discussion of the history of these ideas). The crucial difference between two-dimensional and three-dimensional turbulence is that the former does not have vortex stretching. This means that squared relative vorticity (often called enstrophy), as well as energy, is conserved in inviscid flows. Onsager 
(1949), Lee (1951), Batchelor (1953), and Fjortoft, (1953) were all aware of the strong constraint that this placed on two-dimensional flows. Fjortoft (1953) showed that conservation of energy and squared vorticity leads to the so-called "inverse cascade." That is, in contrast to three-dimensional turbulence, two-dimensional turbulence exhibits an energy cascade to large scales. Charney (1971) showed that potential enstrophy (squared PV) in quasi-geostrophic flows plays a role similar to that of squared relative vorticity in two-dimensional flows. He therefore argued that the inverse cascades of purely two-dimensional flows ought to hold in stratified quasigeostrophic flows as well, even though the latter are not strictly two-dimensional (because stratification leads to the possibility of depth-dependent motions).

If the inverse cascade were fully developed in the mid-ocean, eddies would be at basin scales. Instead, they are much more compact. A possible explanation for this was offered by Rhines (1975), who found that freely evolving two-dimensional flows stopped cascading on a beta plane, at a scale $\sqrt{\frac{V}{\beta}}$, where $U$ is the velocity scale of the turbulence and $\beta$ is planetary beta. Rhines estimated this scale and found it to be in rough agreement with the scales of mid-ocean eddies. However, more recent data (Stammer 1997; figure 1-2) indicates that $R_{d}$, not $\sqrt{\frac{U}{\beta}}$, is the controlling scale of the eddies. Rhines (1977) extended his turbulence studies to two layers. He found that a freely evolving solution on an f-plane did indeed behave like a two-dimensional flow, in that initial vertical structure-i.e. baroclinic energy-was rapidly eroded; as the flow "barotropized", that is, became barotropic, it also cascaded to larger scales. He found that barotropization and the inverse cascade could be halted by introducing a vertical asymmetry into the problem, namely rough bottom topography. He then suggested that rough topography could explain the baroclinicity and compactness of mid-ocean eddies. Bretherton and Haidvogel (1976) also pointed out the importance of rough topography. They used a minimum enstrophy principle to argue that eddies tend towards a state of flowing along topographic contours, which therefore halt the inverse cascade. 
Two important early papers on forced-dissipated geostrophic turbulence were by Salmon $(1978 ; 1980)$. In forced-dissipated runs, energy is continually injected into the deformation radius scale and into the baroclinic mode. Yet, in like manner to Rhines (1977), Salmon found that eddies barotropized and cascaded to larger horizontal scales. The ideas of these and other early authors were extended by Larichev and Held (1995), who found that in homogeneous forced-dissipated flows eddy energy generation is dominated by the large, energetic eddies at the endpoint of the cascade, and not by deformation scale eddies as one would expect from linear instability theory. It is the model of Larichev and Held (1995) that provides the starting point for the numerical work presented here.

\subsection{Contributions of this dissertation}

The main hypothesis of this study is that local baroclinic instability of the weak gyre flows of the mid-ocean contributes substantially to the generation of mid-ocean eddy energy. Our model of the ocean interior is one of forced-dissipated, two-layer, horizontally homogeneous quasi-geostrophic turbulence. The dissertation will primarily focus on mechanisms that control three properties of mid-ocean eddies: 1) eddy kinetic energy is roughly equipartitioned in the vertical between the barotropic and first baroclinic modes (c.f. Wunsch 1997; figures 1-3 and 1-4), 2) horizontal length scales of eddies appear to correlate better with the first baroclinic mode deformation radius than with larger cascade scales (Stammer 1997; figure 1-2), and 3) eddy kinetic energy is much larger than mean kinetic energy (c.f. Gill et al. 1974; figure 1-1). We discuss each of these below. 


\subsubsection{Effects of stratification and friction on vertical struc- ture of eddies}

The barotropization found in the models of Rhines (1977), Salmon (1978) and Salmon (1980), and Larichev and Held (1995) is in conflict with observations, which show an approximate equipartition of energy between barotropic and baroclinic modes (Wunsch 1997; figures 1-3 and 1-4). In both studies of Salmon and in Larichev and Held (1995), the depths of the two layers were equal. Flierl (1978) and Fu and Fierl (1980) argued that models with a thin top layer would retain more energy in the baroclinic field than models with equal layer depths, since in the latter case there is no projection onto the baroclinic mode when two baroclinic modes interact. This will be further developed in later chapters, and complemented with numerical results (note that Flierl (1978) and Fu and Flierl (1980) did not present numerical results). Stratification also affects eddy baroclinicity because the baroclinic mode is weakly damped when the stratification is surface-intensified and the friction occurs only at the bottom. Numerical experiments will be done with two values of the stratifica-

tion parameter $\delta=\frac{H_{1}}{H_{2}}$; experiments with equal layer depths represent a uniform stratification while $\delta=0.2$ experiments represent a surface-trapped stratification. Sets of experiments with equal Ekman spin-down rates in both layers (a case we call "symmetric friction"), as well as sets with Ekman friction in the bottom layer only, will be performed to highlight the importance of the bottom-trapped nature of the dissipation. It will be shown that both the nonlinear projection mechanism and the damping mechanism enhance eddy baroclinicity.

\subsubsection{Effects of stratification and friction on horizontal scales of eddies}

The eddy fields in Salmon (1978), Salmon (1980), and Larichev and Held (1995) tend to be much larger than the first mode deformation radius. As we have seen, this is 
also in conflict with observations. As model eddy fields become more baroclinic with surface-trapped stratification and bottom-trapped friction, their horizontal scales should become closer to the deformation radius. In this dissertation a cascade inequality is developed which quantifies the "two-dimensionality" of equilibrated eddy fields in forced-dissipated flows. The inequality is developed from the energy and enstrophy equations using reasoning similar to that used by Fjortoft (1953) in his pioneering work on two-dimensional turbulence. The inequality forbids eddies to simultaneously contain substantial energy in the baroclinic mode and in scales near $R_{d}$, in the symmetric friction case. Numerical results strongly support this. Bottom friction, especially when coupled with a surface-trapped stratification, allows the inequality to be broken. Therefore, in terms of the measure defined by the inequality, the eddies in this case are simultaneously strongly baroclinic and at scales near $R_{d}$, in accordance with observations.

\subsubsection{Generation of strong eddy fields from nonzonal mean flows on a beta plane}

If we take the following as typical of mid-latitude gyres: $\overline{u_{1}}=1 \mathrm{~cm} \mathrm{~s}^{-1}, \overline{u_{2}}=0, R_{d}=$ $50 \mathrm{~km}$, and $\beta=2 \times 10^{-11} \mathrm{~m}^{-1} \mathrm{~s}^{-1}$, then by the Charney-Stern criterion (Charney and Stern 1962), the mean shear flow is baroclinically stable if it is zonal (either eastward or westward). It would seem therefore that large regions of the ocean interior are not capable of generating a vigorous eddy field in the presence of $\beta$. (Note that Gill et al. 1974 carried out their linear stability analyses on vertical shear flows having velocity differences as large as $4 \mathrm{~cm} \mathrm{~s}^{-1}$, which may be characteristic of strong currents in the interior such as the Atlantic North Equatorial Current, but in general are stronger than mid-ocean gyre flows. They also assumed destabilizing surface temperature gradients.) Time-mean currents of the mid-ocean are usually nonzonal, due to the presence of continental boundaries. As discussed earlier, linear stability analyses of nonzonal mean flows (Robinson and McWilliams 1974; Pedlosky 1987) demonstrate 
that they are inherently more unstable than zonal flows. This thesis contains a systematic study of fully nonlinear geostrophic turbulence forced by nonzonal flows. It is shown that large eddy energies can be generated even when mean PV gradients arising from vertical shear are weak compared with planetary beta. Equilibrated eddy energy is examined as a function of angle for a fixed planetary beta, and as a function of planetary beta for a fixed angle. Anisotropic regimes consisting of slowly drifting zonal jets are found when beta dominates the mean gradients, while a surprising regime of isotropic, strongly baroclinic, monopolar vortices is found to exist when beta and the shear-induced gradients are comparable in magnitude.

\subsection{Overview of dissertation}

This dissertation is organized as follows. Chapter 2 presents the governing PV equations in both layer and modal forms. From these, energy and enstrophy equations are derived. Choice of model parameters, the numerical method used to integrate the model equations, and the nature of the various small-scale dissipation schemes used in the dissertation are also discussed in chapter 2. Finally, chapter 2 concludes with a description of a linear stability analysis which will be useful throughout the remainder of the dissertation. Chapter 3 presents four sets of numerical experiments which explore the effects of stratification and friction on eddy vertical structure and length scales. Chapter 4 presents an inequality which quantifies the effects of stratification and friction on the inverse cascade to the barotropic mode and to scales larger than $R_{d}$. Chapter 5 presents results from a systematic study of geostrophic turbulence forced by nonzonal mean flows. Eddy characteristics are examined as functions of angle of mean shear for a fixed value of beta, and as functions of beta for a fixed angle. The latter exploration uncovered an interesting regime of coherent vortices, which is studied in more detail in chapter 6 . Conclusions are presented in chapter 7 . 


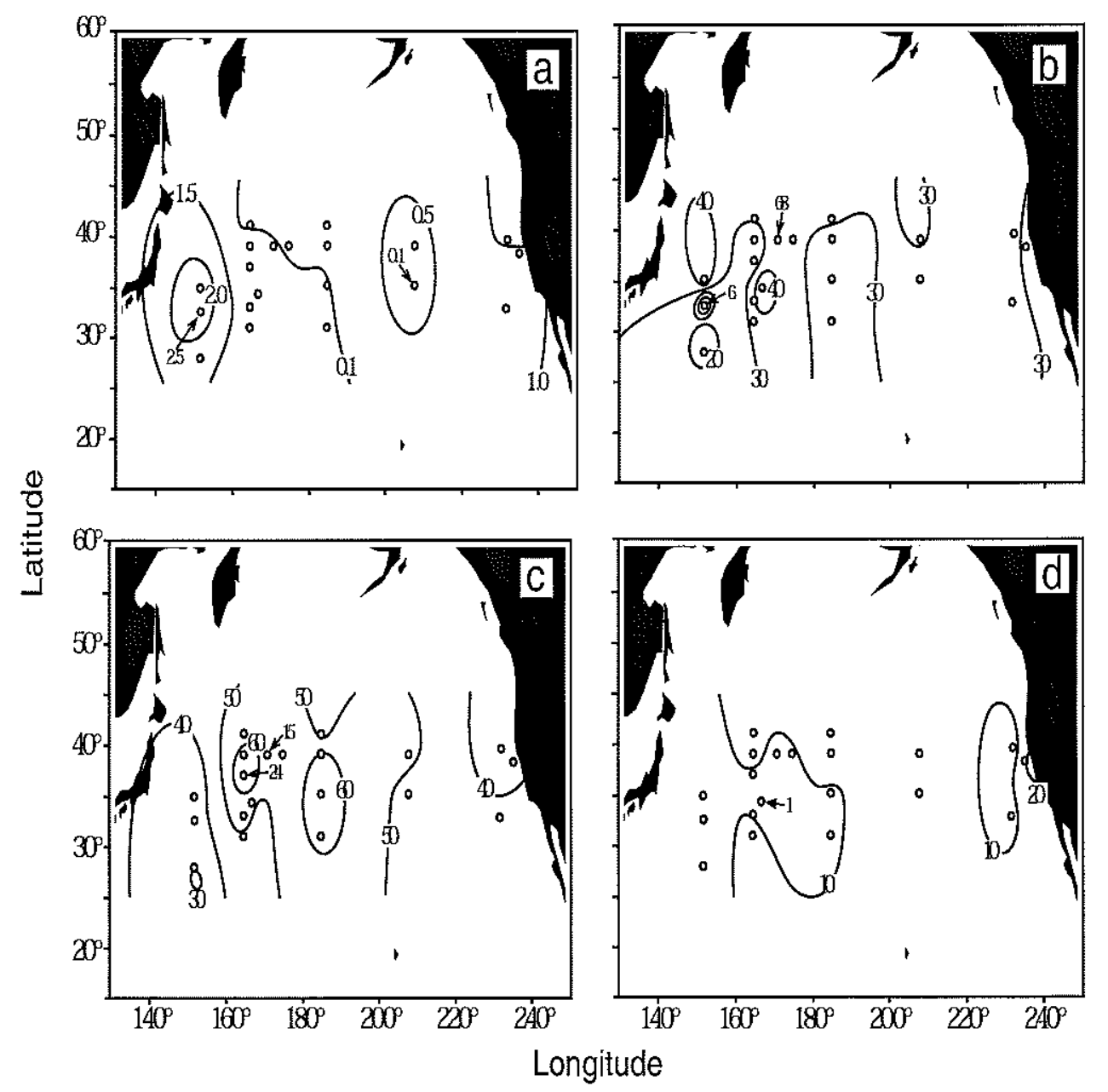

Figure 1-3: (a) $\log _{10}$ of the water column average kinetic energy per unit mass (in $\mathrm{cm}^{2} \mathrm{~s}^{-2}$ ) in the North Pacific Ocean. (b) Percentage of (a) found in the barotropic mode. (c) Percentage of (a) found in the first baroclinic mode. (d) Percentage of (a) found in the second baroclinic mode. Figure taken from Wunsch (1997). 


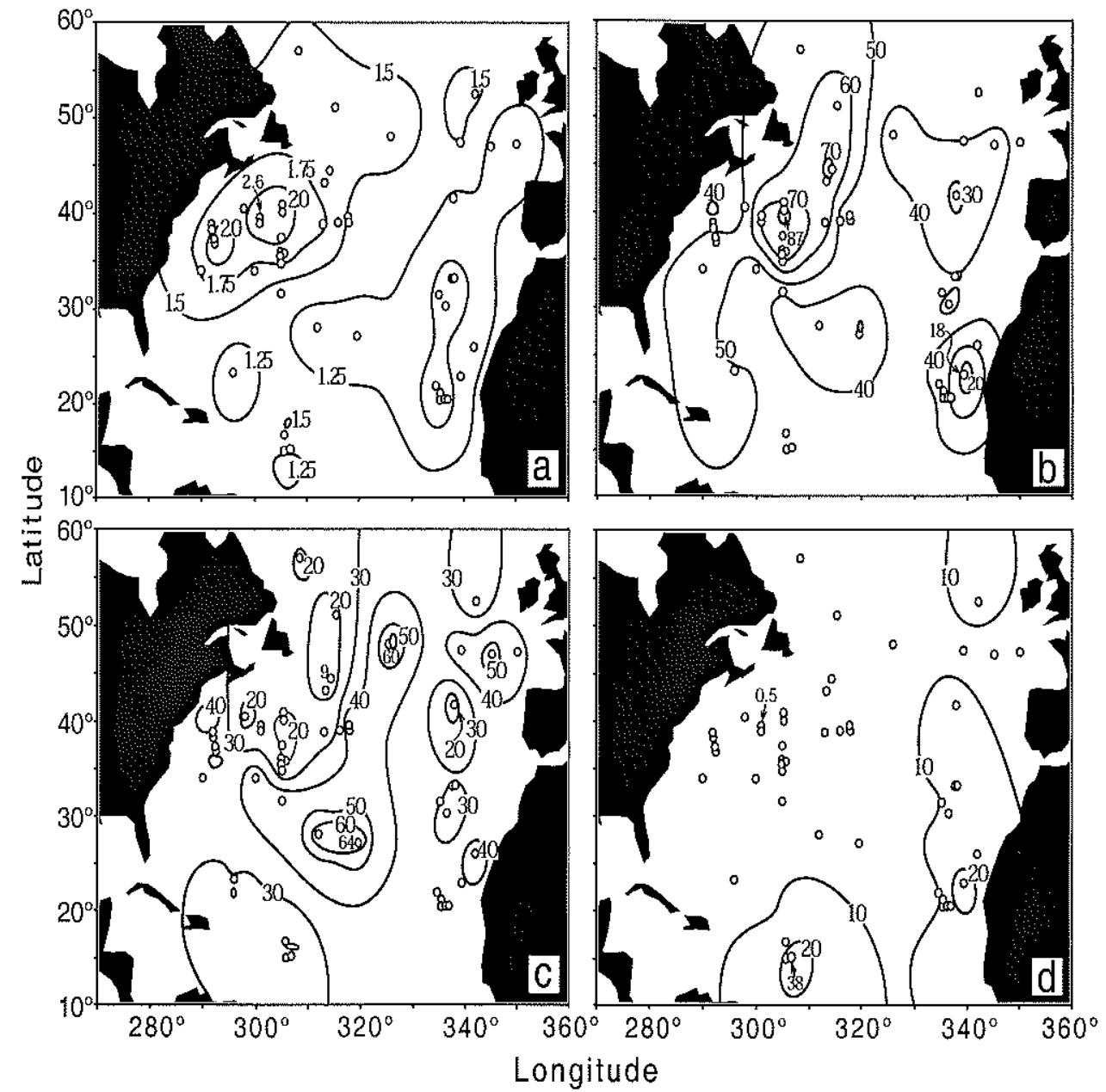

Figure 1-4: Same as in figure 1-3 but for the North Atlantic. Figure taken from Wunsch (1997). 


\section{Chapter 2}

\section{The model}

\subsection{Quasi-geostrophic PV equations}

The numerical results in this thesis arise from integrating a two-layer quasi-geostrophic (QG) model. The two-layer model is the simplest in which to study baroclinic instability. See Pedlosky (1987) for a rigorous discussion and derivation of QG. The two-layer version of QG used here is close to that in Flierl (1978), but with an imposed mean flow. It is similar to that on page 423 of Pedlosky (1987), except that an Ekman friction is allowed to exist in the top layer, there is no bottom topography, and model quantities have been split into imposed time-mean quantities and fluctuation quantities. Another difference will be seen shortly, in the definition of $R_{d}$.

A general form of the two-layer equations, which covers all the cases studied in this thesis, is:

$$
\begin{aligned}
& \frac{\partial q_{1}}{\partial t}+\overline{u_{1}} \frac{\partial q_{1}}{\partial x}+\overline{v_{1}} \frac{\partial q_{1}}{\partial y}-\frac{\partial \overline{q_{1}}}{\partial x} \frac{\partial \psi_{1}}{\partial y}+\frac{\partial \overline{q_{1}}}{\partial y} \frac{\partial \psi_{1}}{\partial x}+J\left(\psi_{1}, q_{1}\right)=-R_{1} \nabla^{2} \psi_{1}+s s d \\
& \frac{\partial q_{2}}{\partial t}+\overline{u_{2}} \frac{\partial q_{2}}{\partial x}+\overline{v_{2}} \frac{\partial q_{2}}{\partial y}-\frac{\partial \overline{q_{2}}}{\partial x} \frac{\partial \psi_{2}}{\partial y}+\frac{\partial \overline{q_{2}}}{\partial y} \frac{\partial \psi_{2}}{\partial x}+J\left(\psi_{2}, q_{2}\right)=-R_{2} \nabla^{2} \psi_{2}+s s d
\end{aligned}
$$


where $\overline{u_{1}}$ and $\overline{u_{2}}$ are the imposed horizontally homogeneous zonal mean flows, $\overline{v_{1}}$ and $\overline{v_{2}}$ are the imposed horizontally homogeneous meridional mean flows, subscripts 1 and 2 denote the upper and lower layers, respectively, $s s d$ stands for small-scale dissipation, to be discussed later in this chapter, and the other quantities are defined below. The model prognostic variables are the fluctuation streamfunctions $\psi_{1}$ and $\psi_{2}$, and the fluctuation potential vorticities $q_{1}$, and $q_{2}$. Fluctuations are defined as deviations from the imposed time-mean quantities. Velocities are defined as derivatives of the streamfunctions:

$$
\begin{aligned}
& \left(u_{1}, v_{1}\right)=\left(-\frac{\partial \psi_{1}}{\partial y}, \frac{\partial \psi_{1}}{\partial x}\right) \\
& \left(u_{2}, v_{2}\right)=\left(-\frac{\partial \psi_{2}}{\partial y}, \frac{\partial \psi_{2}}{\partial x}\right)
\end{aligned}
$$

Nonlinear interactions are contained in the Jacobian term, defined by:

$$
J(A, B)=\frac{\partial A}{\partial x} \frac{\partial B}{\partial y}-\frac{\partial A}{\partial y} \frac{\partial B}{\partial x}
$$

The ratio of the depth of the upper layer, $H_{1}$, to the depth of the lower layer, $\mathrm{H}_{2}$ is denoted by $\delta$ :

$$
\delta=\frac{H_{1}}{H_{2}} .
$$

A $\delta$ value of 1 represents a uniform stratification, while a $\delta$ value less than 1 represents a surface-trapped stratification.

On page 425 of Pedlosky (1987) he defines a deformation radius within each layer. Here we follow Flierl (1978) and define a single deformation radius $R_{d}$ by $\frac{1}{R_{d}^{2}}=F_{1}+F_{2}$, where $F_{1}=\frac{\rho_{0} f_{0}^{2}}{g\left(\rho_{2}-\rho_{1}\right) H_{1}}$ and $F_{2}=\frac{\rho_{0} f_{0}^{2}}{g\left(\rho_{2}-\rho_{1}\right) H_{2}}$. (Note that $f_{0}$ is the 
Coriolis parameter, $g$ is gravitational acceleration, $\rho_{1}$ and $\rho_{2}$ are the densities of the upper and lower layers, respectively, and $\rho_{0}$ is the average density.) We have assumed a rigid lid, so that the barotropic mode deformation radius is infinite. With this definition of $R_{d}$ the fluctuation potential vorticities are:

$$
\begin{array}{r}
q_{1}=\nabla^{2} \psi_{1}+\frac{\left(\psi_{2}-\psi_{1}\right)}{(1+\delta) R_{d}^{2}} \\
q_{2}=\nabla^{2} \psi_{2}+\frac{\delta\left(\psi_{1}-\psi_{2}\right)}{(1+\delta) R_{d}^{2}}
\end{array}
$$

while the mean PV gradients are:

$$
\begin{gathered}
\frac{\partial \overline{q_{1}}}{\partial x}=\frac{\left(\overline{v_{2}}-\overline{v_{1}}\right)}{(1+\delta) R_{d}^{2}} \\
\frac{\partial \overline{q_{1}}}{\partial y}=\beta+\frac{\left(\overline{u_{1}}-\overline{u_{2}}\right)}{(1+\delta) R_{d}^{2}} \\
\frac{\partial \overline{q_{2}}}{\partial x}=\frac{\delta\left(\overline{v_{1}}-\overline{v_{2}}\right)}{(1+\delta) R_{d}^{2}} \\
\frac{\partial \overline{q_{2}}}{\partial y}=\beta+\frac{\delta\left(\overline{u_{2}}-\overline{u_{1}}\right)}{(1+\delta) R_{d}^{2}},
\end{gathered}
$$

where $\beta=\frac{\partial f}{\partial y}$ is the planetary PV gradient.

The layer Ekman friction coefficients $R_{1}$ and $R_{2}$ have units of inverse time. The lower layer Ekman friction arises from contact of the water with the sea bottom. The upper layer Ekman friction is not thought of as having a physical origin. It will be argued in this thesis that the bottom-trapped nature of the friction, i.e. the fact 
that the friction does occur at the bottom rather than throughout the fluid, is very important. This can be shown by comparing experiments with bottom-friction only to experiments with "symmetric friction", that is, experiments in which $R_{1}=R_{2}$. Note that $R_{2}$ is a function of the depth of the Ekman layer:

$$
R_{2}=\frac{f_{0} d_{E k m a n}}{2 H_{2}},
$$

where $f_{0}$ is the Coriolis parameter and

$$
d_{\text {Ekman }}=\sqrt{\frac{2 \nu_{\text {turbulent }}}{f_{0}}},
$$

$\nu_{\text {turbulent }}$ being the turbulent eddy viscosity in the bottom Ekman layer (c.f. Pedlosky 1987; Flierl 1978). If $\nu_{\text {turbulent }}$ were the same in both top and bottom layers, then with the rigid lid present, $R_{1}$ would differ from $R_{2}$ by a factor of $\delta$. We will see later, however, that $R_{1}=R_{2}$ is the most appropriate choice to make for a friction occuring throughout the fluid, in terms of highlighting the importance of bottom friction with a contrasting set of experiments.

\subsubsection{Modal PV equations}

The numerical model integrates the layer equations, but some aspects of interpretation are easier in a modal framework (c.f. Pedlosky 1987; Flierl 1978). The layer PV equations can be transformed into modal equations using the following definitions, in which the subscripts BT and BC denote barotropic and baroclinic modes, respectively:

$$
\psi_{B T}=\frac{\delta \psi_{1}+\psi_{2}}{1+\delta}
$$




$$
\begin{gathered}
\psi_{B C}=\frac{\sqrt{\delta}\left(\psi_{1}-\psi_{2}\right)}{1+\delta} \\
q_{B T}=\frac{\delta q_{1}+q_{2}}{1+\delta}=\nabla^{2} \psi_{B T} \\
q_{B C}=\frac{\sqrt{\delta}\left(q_{1}-q_{2}\right)}{1+\delta}=\nabla^{2} \psi_{B C}-\frac{1}{R_{d}^{2}} \psi_{B C} .
\end{gathered}
$$

Note the association of the baroclinic mode with $R_{d}$ in 2.17 . With these definitions, the barotropic PV equation is:

$$
\begin{gathered}
\frac{\partial q_{B T}}{\partial t}+\frac{\delta \overline{u_{1}}+\overline{u_{2}}}{1+\delta} \frac{\partial q_{B T}}{\partial x}+\frac{\sqrt{\delta}\left(\overline{u_{1}}-\overline{u_{2}}\right)}{1+\delta} \frac{\partial}{\partial x} \nabla^{2} \psi_{B C} \\
+\frac{\delta \overline{v_{1}}+\overline{v_{2}}}{1+\delta} \frac{\partial q_{B T}}{\partial y}+\frac{\sqrt{\delta}\left(\overline{v_{1}}-\overline{v_{2}}\right)}{1+\delta} \frac{\partial}{\partial y} \nabla^{2} \psi_{B C} \\
+\beta \frac{\partial \psi_{B T}}{\partial x}+J\left(\psi_{B T}, \nabla^{2} \psi_{B T}\right)+J\left(\psi_{B C}, \nabla^{2} \psi_{B C}\right) \\
=-\frac{\delta R_{1}+R_{2}}{1+\delta} \nabla^{2} \psi_{B T}+\frac{\sqrt{\delta}\left(R_{2}-R_{1}\right)}{1+\delta} \nabla^{2} \psi_{B C}+s s d
\end{gathered}
$$

while the baroclinic PV equation is:

$$
\begin{gathered}
\frac{\partial q_{B C}}{\partial t}+\frac{\sqrt{\delta}\left(\overline{u_{1}}-\overline{u_{2}}\right)}{1+\delta} \frac{\partial}{\partial x} q_{B T}+\frac{\overline{u_{1}}+\delta \overline{u_{2}}}{1+\delta} \frac{\partial q_{B C}}{\partial x} \\
+\frac{\sqrt{\delta}\left(\overline{v_{1}}-\overline{v_{2}}\right)}{1+\delta} \frac{\partial}{\partial y} q_{B T}+\frac{\overline{v_{1}}+\delta \overline{v_{2}}}{1+\delta} \frac{\partial q_{B C}}{\partial y}
\end{gathered}
$$




$$
\begin{gathered}
+\frac{\sqrt{\delta}\left(\overline{u_{1}}-\overline{u_{2}}\right)}{(1+\delta) R_{d}^{2}} \frac{\partial \psi_{B T}}{\partial x}+\frac{1-\delta \overline{u_{1}}-\overline{u_{2}}}{1+\delta} \frac{\partial \psi_{B C}}{\partial x} \\
+\frac{\sqrt{\delta}\left(\overline{v_{1}}-\overline{v_{2}}\right)}{(1+\delta) R_{d}^{2}} \frac{\partial \psi_{B T}}{\partial y}+\frac{1-\delta}{1+\delta} \frac{\overline{v_{1}}-\overline{v_{2}}}{R_{d}^{2}} \frac{\partial \psi_{B C}}{\partial y} \\
+\beta \frac{\partial \psi_{B C}}{\partial x}+J\left(\psi_{B T}, q_{B C}\right)+J\left(\psi_{B C}, q_{B T}\right)+\frac{1-\delta}{\sqrt{\delta}} J\left(\psi_{B C}, \nabla^{2} \psi_{B C}\right) \\
=\frac{\sqrt{\delta}\left(R_{2}-R_{1}\right)}{1+\delta} \nabla^{2} \psi_{B T}-\frac{R_{1}+\delta R_{2}}{1+\delta} \nabla^{2} \psi_{B C}+s s d .
\end{gathered}
$$

\subsection{Energy and enstrophy equations}

The energy and enstrophy equations are shown below in both layer and modal formulations. Both will prove useful in interpreting later results.

\subsubsection{Layer energy and enstrophy equations}

Layer energy equations are derived by multiplying the top and bottom layer PV equations 2.1 and 2.2 by $\frac{\delta \psi_{1}}{1+\delta}$ and $\frac{\psi_{2}}{1+\delta}$ respectively, and integrating over the domain (c.f. Charney 1971). Many of the terms, including the Jacobian terms, integrate to zero because of the doubly periodic boundary conditions.

The top layer energy equation is:

$$
\begin{gathered}
\frac{\partial}{\partial t} \iint \frac{1}{2} \frac{\delta\left(\nabla \psi_{1}\right)^{2}}{1+\delta} d x d y+\iint \delta \psi_{1} \frac{\partial}{\partial t} \frac{\left(\psi_{1}-\psi_{2}\right)}{(1+\delta)^{2} R_{d}^{2}} d x d y \\
+\frac{\delta \overline{u_{1}}}{1+\delta} \iint q_{1} \frac{\partial}{\partial x} \psi_{1} d x d y+\frac{\delta \overline{v_{1}}}{1+\delta} \iint q_{1} \frac{\partial}{\partial y} \psi_{1} d x d y \\
=-\frac{\delta R_{1}}{1+\delta} \iint\left(\nabla \psi_{1}\right)^{2} d x d y+s s d
\end{gathered}
$$


while the bottom layer energy equation is:

$$
\begin{gathered}
\frac{\partial}{\partial t} \iint \frac{1}{2} \frac{\left(\nabla \psi_{2}\right)^{2}}{1+\delta} d x d y-\delta \iint \psi_{2} \frac{\partial}{\partial t} \frac{\left(\psi_{1}-\psi_{2}\right)}{(1+\delta)^{2} R_{d}^{2}} d x d y \\
+\frac{\overline{u_{2}}}{1+\delta} \iint q_{2} \frac{\partial}{\partial x} \psi_{2} d x d y+\frac{\overline{v_{2}}}{1+\delta} \iint q_{2} \frac{\partial}{\partial y} \psi_{2} d x d y \\
=-\frac{R_{2}}{1+\delta} \iint\left(\nabla \psi_{2}\right)^{2} d x d y+s s d .
\end{gathered}
$$

Adding these yields a total energy equation:

$$
\begin{array}{r}
\frac{\partial}{\partial t} \iint \frac{1}{2}\left[\frac{\delta\left(\nabla \psi_{1}\right)^{2}}{1+\delta}+\frac{\left(\nabla \psi_{2}\right)^{2}}{1+\delta}+\frac{\delta\left(\psi_{1}-\psi_{2}\right)^{2}}{(1+\delta)^{2} R_{d}^{2}}\right] d x d y \\
+\frac{\delta \overline{u_{1}}}{1+\delta} \iint q_{1} \frac{\partial}{\partial x} \psi_{1} d x d y+\frac{\delta \overline{v_{1}}}{1+\delta} \iint q_{1} \frac{\partial}{\partial y} \psi_{1} d x d y \\
+\frac{\overline{u_{2}}}{1+\delta} \iint q_{2} \frac{\partial}{\partial x} \psi_{2} d x d y+\frac{\overline{v_{2}}}{1+\delta} \iint q_{2} \frac{\partial}{\partial y} \psi_{2} d x d y \\
=-\frac{\delta R_{1}}{1+\delta} \iint\left(\nabla \psi_{1}\right)^{2} d x d y-\frac{R_{2}}{1+\delta} \iint\left(\nabla \psi_{2}\right)^{2} d x d y+s s d
\end{array}
$$

Simple manipulations yield the constraint that vertically integrated PV fluxes are zero in a homogeneous flow:

$$
\begin{aligned}
& H_{1} \iint q_{1} \frac{\partial}{\partial x} \psi_{1} d x d y+H_{2} \iint q_{2} \frac{\partial}{\partial x} \psi_{2} d x d y=0 \\
& H_{1} \iint q_{1} \frac{\partial}{\partial y} \psi_{1} d x d y+H_{2} \iint q_{2} \frac{\partial}{\partial y} \psi_{2} d x d y=0
\end{aligned}
$$

This allows the total energy equation to be rewritten as: 


$$
\begin{gathered}
\frac{\partial}{\partial t} \iint \frac{1}{2}\left[\frac{\delta\left(\nabla \psi_{1}\right)^{2}}{1+\delta}+\frac{\left(\nabla \psi_{2}\right)^{2}}{1+\delta}+\frac{\delta\left(\psi_{1}-\psi_{2}\right)^{2}}{(1+\delta)^{2} R_{d}^{2}}\right] d x d y \\
+\frac{\delta\left(\overline{u_{1}}-\overline{u_{2}}\right)}{1+\delta} \iint q_{1} \frac{\partial}{\partial x} \psi_{1} d x d y+\frac{\delta\left(\overline{v_{1}}-\overline{v_{2}}\right)}{1+\delta} \iint q_{1} \frac{\partial}{\partial y} \psi_{1} d x d y \\
=-\frac{\delta R_{1}}{1+\delta} \iint\left(\nabla \psi_{1}\right)^{2} d x d y-\frac{R_{2}}{1+\delta} \iint\left(\nabla \psi_{2}\right)^{2} d x d y+s s d,
\end{gathered}
$$

from which we see that eddy PV fluxes extract energy from a homogeneous mean flow only if it is vertically sheared. In a statistical equilibrium, the $\frac{\partial}{\partial t}$ terms go to zero in a time average, and energy generation is balanced by dissipation. Ekman friction in either layer is a potential sink of energy, as is small-scale dissipation.

In both individual layer energy equations, the second term on the left-hand side prevents one from identifying terms as $\frac{\partial}{\partial t} L a y e r E n e r g y$-in other words, there is no "layer one energy" or "layer two energy" that is separately conserved. Only when the two layer equations are added do these terms combine to form a total energy term which is conserved in the absence of forcing and dissipation. Because energy can be transferred between layers, we will see later that bottom Ekman friction can dominate dissipation of total energy in experiments where Ekman friction is absent in the upper layer. Even when small-scale dissipation is the only dissipation present in the upper layer, its effects on the energy balance are usually minimal, as we shall see.

Potential enstrophy equations (Charney 1971) are obtained by multiplying the layer PV equations by $\frac{\delta q_{1}}{1+\delta}$ and $\frac{q_{2}}{1+\delta}$ respectively, and again integrating over the domain. The top layer potential enstrophy equation is:

$$
\frac{\partial}{\partial t} \iint \frac{1}{2} \frac{\delta q_{1}^{2}}{1+\delta} d x d y
$$




$$
\begin{gathered}
+\frac{\delta}{1+\delta}\left(\beta+\frac{\overline{u_{1}}-\overline{u_{2}}}{(1+\delta) R_{d}^{2}}\right) \iint q_{1} \frac{\partial}{\partial x} \psi_{1} d x d y+\frac{\delta\left(\overline{v_{1}}-\overline{v_{2}}\right)}{(1+\delta)^{2} R_{d}^{2}} \iint q_{1} \frac{\partial}{\partial y} \psi_{1} d x d y \\
=-\frac{\delta R_{1}}{1+\delta} \iint\left(\nabla^{2} \psi_{1}\right)^{2} d x d y-\frac{\delta R_{1}}{(1+\delta)^{2} R_{d}^{2}} \iint\left(\nabla \psi_{1}\right)^{2} d x d y \\
+\frac{\delta R_{1}}{(1+\delta)^{2} R_{d}^{2}} \iint \nabla \psi_{1} \bullet \nabla \psi_{2} d x d y+s s d
\end{gathered}
$$

while the bottom layer potential enstrophy equation is:

$$
\begin{gathered}
\frac{\partial}{\partial t} \iint \frac{1}{2} \frac{q_{2}^{2}}{1+\delta} d x d y \\
+\frac{1}{1+\delta}\left(\beta-\frac{\delta\left(\overline{u_{1}}-\overline{u_{2}}\right)}{(1+\delta) R_{d}^{2}}\right) \iint q_{2} \frac{\partial}{\partial x} \psi_{2} d x d y-\frac{\delta\left(\overline{v_{1}}-\overline{v_{2}}\right)}{(1+\delta)^{2} R_{d}^{2}} \iint q_{2} \frac{\partial}{\partial y} \psi_{2} d x d y \\
=-\frac{R_{2}}{1+\delta} \iint\left(\nabla^{2} \psi_{2}\right)^{2} d x d y-\frac{\delta R_{2}}{(1+\delta)^{2} R_{d}^{2}} \iint\left(\nabla \psi_{2}\right)^{2} d x d y \\
+\frac{\delta R_{2}}{(1+\delta)^{2} R_{d}^{2}} \iint \nabla \psi_{1} \bullet \nabla \psi_{2} d x d y+s s d
\end{gathered}
$$

A total potential enstrophy equation is obtained by adding these:

$$
\begin{gathered}
\frac{\partial}{\partial t} \iint \frac{1}{2}\left[\frac{\delta q_{1}^{2}+q_{2}^{2}}{1+\delta}\right] d x d y \\
+\frac{\delta\left(\overline{u_{1}}-\overline{u_{2}}\right)}{(1+\delta) R_{d}^{2}} \iint q_{1} \frac{\partial \psi_{1}}{\partial x} d x d y+\frac{\delta\left(\overline{v_{1}}-\overline{v_{2}}\right)}{(1+\delta) R_{d}^{2}} \iint q_{1} \frac{\partial \psi_{1}}{\partial y} d x d y \\
=-\frac{\delta R_{1}}{1+\delta} \iint\left(\nabla^{2} \psi_{1}\right)^{2} d x d y-\frac{\delta R_{1}}{(1+\delta)^{2} R_{d}^{2}} \iint\left(\nabla \psi_{1}\right)^{2} d x d y \\
-\frac{R_{2}}{1+\delta} \iint\left(\nabla^{2} \psi_{2}\right)^{2} d x d y-\frac{\delta R_{2}}{(1+\delta)^{2} R_{d}^{2}} \iint\left(\nabla \psi_{2}\right)^{2} d x d y
\end{gathered}
$$




$$
+\frac{\delta\left(R_{1}+R_{2}\right)}{(1+\delta)^{2} R_{d}^{2}} \iint \nabla \psi_{1} \bullet \nabla \psi_{2} d x d y+s s d
$$

where we have made use of 2.23 to eliminate the $\beta$ terms.

In contrast to the energies, layer potential enstrophies are individually conserved in the absence of forcing and dissipation (and beta). On the f-plane, there is no means for layer one potential enstrophy to be transferred downwards to layer two. Therefore, the dissipation of layer one enstrophy must be accomplished by the smallscale dissipation within the top layer itself. Consequently, we anticipate that smallscale dissipation will play a larger role in the enstrophy balance than in the energy balance. Note that a nonzero $\beta$ allows enstrophy transfer between layers. Even in the absence of forcing and dissipation, enstrophy may not be conserved in each layer in that case, but total enstrophy integrated over both layers will still be conserved.

\subsubsection{Modal energy and enstrophy equations}

The barotropic and baroclinic PV equations can be multiplied by $\psi_{B T}, \psi_{B C}, q_{B T}$, and $q_{B C}$, and integrated over the domain to yield barotropic and baroclinic energy and enstrophy equations. Adding these gives modal versions of the total energy equation:

$$
\begin{gathered}
\frac{\partial}{\partial t} \iint \frac{1}{2}\left[\left(\nabla \psi_{B T}\right)^{2}+\left(\nabla \psi_{B C}\right)^{2}+\frac{\psi_{B C}^{2}}{R_{d}^{2}}\right] d x d y \\
+\frac{\sqrt{\delta}\left(\overline{u_{1}}-\overline{u_{2}}\right)}{1+\delta} \iint\left[q_{B C} \frac{\partial \psi_{B T}}{\partial x}+q_{B T} \frac{\partial \psi_{B C}}{\partial x}\right] d x d y \\
+\frac{\sqrt{\delta}\left(\overline{v_{1}}-\overline{v_{2}}\right)}{1+\delta} \iint\left[q_{B C} \frac{\partial \psi_{B T}}{\partial y}+q_{B T} \frac{\partial \psi_{B C}}{\partial y}\right] d x d y \\
=-\left(\frac{\delta R_{1}+R_{2}}{1+\delta}\right) \iint\left(\nabla \psi_{B T}\right)^{2} d x d y-\left(\frac{R_{1}+\delta R_{2}}{1+\delta}\right) \iint\left(\nabla \psi_{B C}\right)^{2} d x d y
\end{gathered}
$$




$$
+\frac{2 \sqrt{\delta}\left(R_{2}-R_{1}\right)}{1+\delta} \iint \nabla \psi_{B T} \bullet \nabla \psi_{B C} d x d y+s s d
$$

as well as the total potential enstrophy equation:

$$
\begin{gathered}
\frac{\partial}{\partial t} \iint \frac{1}{2}\left[q_{B T}^{2}+q_{B C}^{2}\right] d x d y \\
+\frac{\sqrt{\delta}\left(\overline{u_{1}}-\overline{u_{2}}\right)}{(1+\delta) R_{d}^{2}} \iint\left[q_{B C} \frac{\partial \psi_{B T}}{\partial x}+q_{B T} \frac{\partial \psi_{B C}}{\partial x}\right] d x d y \\
+\frac{\sqrt{\delta}\left(\overline{v_{1}}-\overline{v_{2}}\right)}{(1+\delta) R_{d}^{2}} \iint\left[q_{B C} \frac{\partial \psi_{B T}}{\partial y}+q_{B T} \frac{\partial \psi_{B C}}{\partial y}\right] d x d y \\
=\frac{\sqrt{\delta}\left(R_{2}-R_{1}\right)}{(1+\delta) R_{d}^{2}} \iint \nabla \psi_{B T} \bullet \nabla \psi_{B C} d x d y+\frac{2 \sqrt{\delta}\left(R_{2}-R_{1}\right)}{1+\delta} \iint \nabla^{2} \psi_{B T} \nabla^{2} \psi_{B C} d x d y \\
-\frac{\delta R_{1}+R_{2}}{1+\delta} \iint\left(\nabla^{2} \psi_{B T}\right)^{2} d x d y-\frac{R_{1}+\delta R_{2}}{1+\delta} \iint\left(\nabla^{2} \psi_{B C}\right)^{2} d x d y \\
-\frac{R_{1}+\delta R_{2}}{(1+\delta) R_{d}^{2}} \iint\left(\nabla \psi_{B C}\right)^{2} d x d y+s s d .
\end{gathered}
$$

Note that with symmetric Ekman friction present $\left(R_{1}=R_{2}\right)$, dissipation terms involving correlations between barotropic and baroclinic modes disappear. One way to interpret the correlation terms in the energy equation is the following: suppose that there is kinetic energy in both the barotropic and baroclinic modes, but that these modes are correlated in such a way as to render the bottom layer velocity zero. If Ekman friction is present only in the bottom layer $\left(R_{1}=0\right)$, then the energy dissipation must sum to zero. The sign-indefinite correlation terms on the right-hand side of equation 2.29 are the means to offset the sign-definite terms and bring the total sum to zero in such a case.

When $R_{1}=R_{2}$, equation 2.29 indicates that barotropic and baroclinic kinetic energies are damped at equal rates, independent of the value of $\delta$. However, when 
Ekman friction occurs only in the bottom layer, the baroclinic kinetic energy is damped at a rate proportional to $\delta$ times the damping rate of barotropic kinetic energy. Therefore, in the bottom-friction only case with equal layer depths, the modes are damped at equal rates; under the action of bottom friction and surfacetrapped stratification, the baroclinic mode is weakly damped. Similar arguments apply to Ekman dissipation of enstrophy.

\subsection{Model parameters}

For almost all of the forced-dissipated runs presented in this thesis, the magnitude of the difference in imposed mean flow velocities between the two layers is $1 \mathrm{~cm} \mathrm{~s}^{-1}$ :

$$
\sqrt{\left(\overline{u_{1}}-\overline{u_{2}}\right)^{2}+\left(\overline{v_{1}}-\overline{v_{2}}\right)^{2}}=1 \mathrm{~cm} \mathrm{~s}^{-1} .
$$

This was chosen as a representative value of mid-ocean mean flows by assuming that transport in the Gulf Stream ( $\sim 50 \mathrm{~km}$ width, $\sim 1 \mathrm{~m} \mathrm{~s}^{-1}$ flow) is balanced by an interior transport of $\sim 1 \mathrm{~cm} \mathrm{~s}^{-1}$ across a basin width of $\sim 5000 \mathrm{~km}$. Tables of eastern North Atlantic current meter data in Muller and Siedler (1992) indicate that our chosen value of mean shear flow may be on the low side, even for that relatively quiescent region of the ocean. However, figure $2-1$ indicates that $1 \mathrm{~cm} \mathrm{~s}^{-1}$ is representative of large regions of the mid-ocean.

The first baroclinic mode deformation radius in all model runs is $50 \mathrm{~km}$, a common value in subtropical gyre regions (c.f. Richman 1976). The domain size in the runs is $20 \pi R_{d} \sim 3000 \mathrm{~km}$. This is too large to truly represent a patch of ocean over which mean flows change very little, and too small to represent a basin. The domain size was chosen to contain many deformation radii so that an inverse cascade away from the deformation scale could be resolved.

Many of the runs are done on an f-plane, in which planetary $\beta$ is zero. Since f-planes are rotationally invariant, the direction of the imposed mean flow on an 


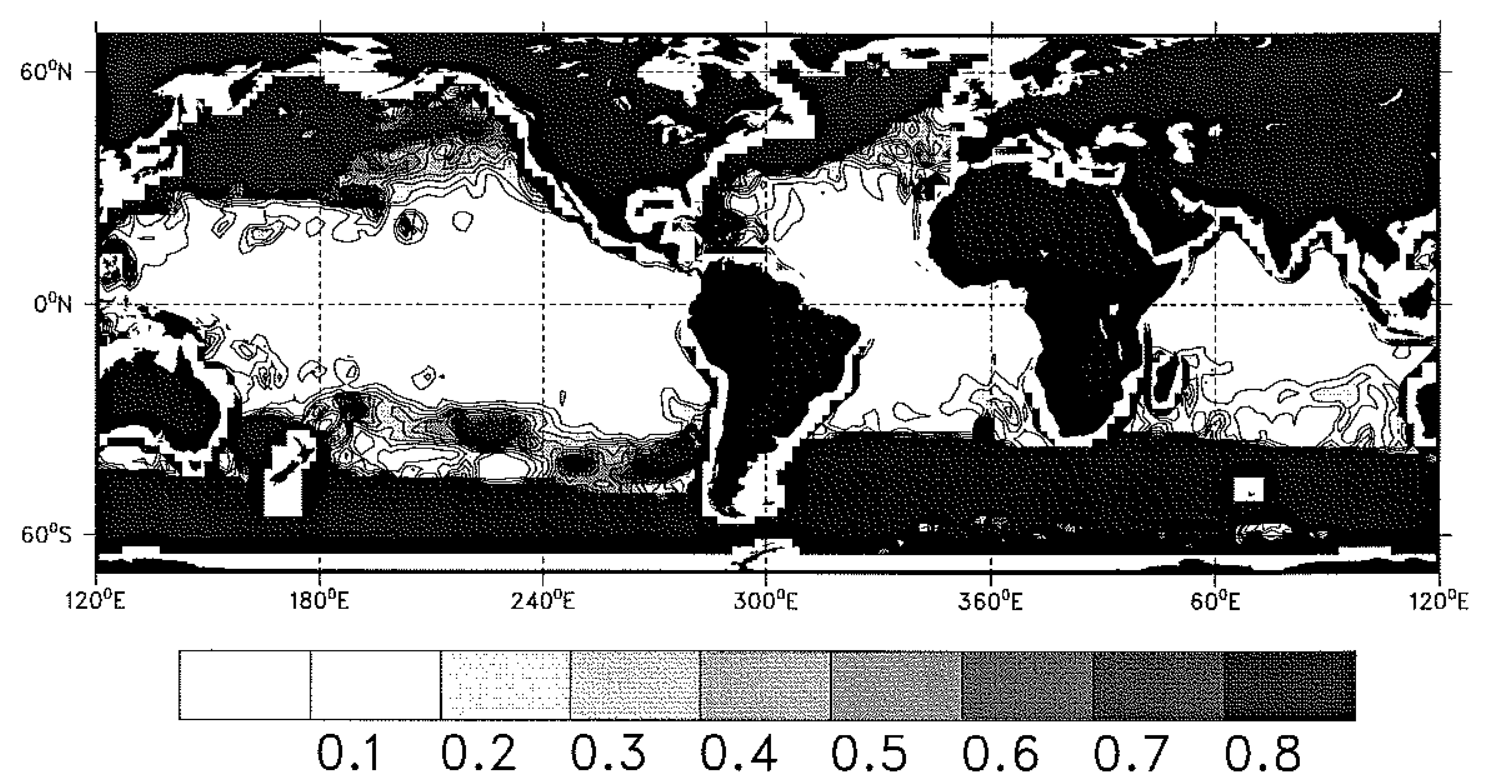

Figure 2-1: Surface kinetic energy of the mean flow, estimated from altimetry data, multiplied by $\sin ^{2}$ (latitude). Units are $\mathrm{cm}^{2} \mathrm{~s}^{-2}$. The difference between this plot and the top panel of 1-1 is that the contour scale has changed drastically, to give more definition to the region of weak interior mean flows. (Courtesy of Carl Wunsch and Charmaine King, personal communication).

f-plane is irrelevant; following tradition, we make it zonal. (In this case, $\overline{u_{1}}=1 \mathrm{~cm}$ $\mathrm{s}^{-1}$, and all other mean velocities are zero). The nonzonal runs will be done on beta planes, using various values of beta. The nominal value of planetary beta will be $2 \cdot 10^{-11} \mathrm{~m}^{-1} \mathrm{~s}^{-1}$.

\subsection{Numerical method}

The two-layer equations are integrated numerically in the so-called "qgb" model, originally written by Bill Dewar and later modified by Glenn Flierl. It has been used by many investigators and gone through many revisions. The author does not know of any formal references for the model, although Miles Sundermeyer has written a helpful unpublished document. The model is written in fortran 77, in modular form. The modular form isolates key steps of the model, for instance, definitions of com- 
mon block variables, timestepping routines, reading of initial conditions, outputting diagnostics, etc., into separate subroutines. This makes it simpler to implement changes. The code was continually modified as the dissertation work progressed.

The qgb code is "pseudo-spectral" (c.f. Canuto et al. 1988 and references therein). Variables are written as Fourier sums, for example:

$$
q(x, y)=\sum_{k} \sum_{l} \hat{q}(k, l) e^{i k x+i l y},
$$

where $\hat{q}(k, l)$ is the Fourier coefficient. The quantity that is time-stepped in the qgb code is $\hat{q}(k, l, t)$ rather than $q(x, y, t)$. Inversions of $\hat{q}(k, l)$ are performed at each time step to obtain streamfunction $\hat{\psi}(k, l)$. A major advantage of the spectral method is that differentiation is accomplished by simple multiplication, for example:

$$
\frac{\partial}{\partial x} q(x, y)=\sum_{k} \sum_{l} i k \hat{q}(k, l) e^{i k x+i l y} .
$$

The nonlinear term $J(\psi, q)$ is evaluated as $\nabla \bullet \overrightarrow{\mathbf{u}} q ; \overrightarrow{\mathbf{u}}$ and $q$ are calculated from their Fourier coefficients via an inverse transform, and then multiplied together. The components $u q$ and $v q$ are transformed, multiplied by $i k$ and $i l$, respectively, then retransformed and added to obtain $\nabla \bullet \overrightarrow{\mathbf{u}} q$. A simple truncation at the Nyquist wavenumbers was employed to eliminate the worst effects of aliasing. More sophisticated methods of spectral de-aliasing were tested by LaCasce (1996) and found to make little difference. The spectral nature of the model automatically makes the boundary conditions doubly periodic, as desired. As suggested by Geoff Vallis, most later versions of the model employed the "fftw" fast fourier transform (http://www.fftw.org). On most platforms the version of the code using fftw was nearly twice as fast as the version of the code having the original transform routine.

The first two time-steps of all model runs are second-order Runge-Kutta; thereafter, the timestepping scheme is third-order Adams-Bashforth, a scheme which is 
accurate and stable but not overly expensive computationally. Streamfunction and PV fields, as well as a number of diagnostics, are written out at regular intervals specified by the user.

Hundreds of exploratory runs were done at a resolution of 64 by 64 grid points. At this resolution, with the parameters given above, there is only one grid point per deformation radius, seemingly quite marginal. Once the 64 by 64 explorations were completed, extensions of selected low resolution runs were made in higher resolution (usually 256 by 256 , or four points per deformation radius). The qualitative, and sometimes even the quantitative, results of the 64-squared runs held up in most cases, although there were some notable exceptions. Because the higher resolution runs are computationally expensive, in many cases shortcuts were employed to save time. For instance, many of the 256 by 256 solutions were initialized with interpolated versions of the equilibrated 64 by 64 solutions, rather than with the small-scale random conditions used to initialize the low-resolution runs; this eliminated computing long spin-up phases at high resolution. Another shortcut employed, when exploring highresolution behaviors as a function of Ekman friction, was to use an equilibrated 256 squared solution with one value of friction as an initial condition for a solution with a nearby value of friction. Again, this took much less time than starting from a small-amplitude, small-scale random initial condition. An implicit assumption here is that forced-dissipated solutions do not depend strongly on initial condition. This is not the case for the vortex regime discussed in chapter 6 , but seems to be true for the rest of the experiments described in the thesis.

\subsection{Small-scale dissipation}

It has long been known that enstrophy cascades to small scales in quasi-geostrophic turbulence (c.f. Salmon 1998). As a result, if there is no small-scale dissipation, we expect enstrophy to rapidly build up at small scales and the model to become 
numerically unstable. The most common small-scale dissipation scheme used in geophysical turbulence studies has been so-called "hyperviscosity", which is of the form:

$$
s s d=(-1)^{n+1} \nu \nabla^{2 n} q_{N},
$$

where $\mathrm{N}$ is either 1 or 2 , depending on layer. Hyperviscosity was used to generate some of the results in chapters 5 and 6 . All other results presented in this dissertation employed an exponential cutoff wavenumber filter, except for a few experiments discussed below in which no small-scale dissipation was needed.

\subsubsection{Large symmetric friction case: no small-scale dissipa- tion needed}

Ekman friction is not scale-selective. It acts on all scales, including the smallest scales present. It was found, therefore, that no small-scale dissipation was needed in the highly viscous symmetric friction runs. These appear to exist in a weakly nonlinear wave regime rather than a strongly turbulent state, so cascades of enstrophy to small scales are slow and can be absorbed by Ekman friction alone. Note that when Ekman friction is in the bottom layer only, small-scale dissipation must be present, to absorb the enstrophy cascade.

The particular implementations of cutoff filter and hyperviscosity employed in this work are described in the following sections.

\subsubsection{Implementation of wavenumber cut-off filter}

In a spectral model, a wavenumber cut-off filter (c.f. Canuto et al. 1988) can be employed which dampens small scales but has no effect on large scales. Here we use a cut-off filter of the form: 


$$
\begin{gathered}
\text { filter }(i, j)=\exp \left(-\alpha\left(\sqrt{x_{\phi}^{2}+y_{\phi}^{2}}-c_{\phi}\right)^{M}\right) \quad \text { where } \quad \sqrt{x_{\phi}^{2}+y_{\phi}^{2}}>c_{\phi} \\
\text { filter }(i, j)=1.0 \quad \text { where } \quad \sqrt{x_{\phi}^{2}+y_{\phi}^{2}} \leq c_{\phi}
\end{gathered}
$$

In LaCasce (1996), $\alpha=-18.4$ and $\mathrm{M}=4$. This author inherited a version of the code with $\alpha=-18$ and $M=7$; these values were used for the initial exploratory runs at 64 by 64 resolution. The LaCasce (1996) values were used later in the thesis, in experiments with 128 squared and 256 squared resolutions.

In the model filter implementation, $\left(x_{\phi}, y_{\phi}\right) \equiv(k \Delta x, l \Delta y)$, where $k$ and $l$ are the zonal and meridional wavenumbers, respectively, in spectral space and $\Delta x=\Delta y$ are the grid spacings. This means that $x_{\phi}$ and $y_{\phi}$ each run from 0 to $\pi$, where $\pi$ corresponds to the Nyquist scale. The cut-off value, $c_{\phi}$, was chosen to be $0.65 \pi$ in the LaCasce thesis as well as in all of the runs in this thesis. The filter and truncation operations both act to prevent accumulation of spectral energy on small scales. Each operation takes place during every model timestep.

Figure 2-2 plots filter values for the three resolutions most commonly used in the thesis. Note that the filter begins to act at wavelengths of $\sim 3 R_{d}, 1.5 R_{d}$, and $0.75 R_{d}$, respectively, for the 64 squared, 128 squared, and 256 squared experiments. This suggests that the 64 squared experiments are marginally resolved. However, it will be shown later that the first moment of the kinetic energy spectrum, a commonly used measure of eddy length scales, is expected to generally be less than or equal to the wavenumber corresponding to the deformation scale. This means that the wavelength of the most energetic scale will be greater than or equal to $2 \pi R_{d}$, not $R_{d}$ itself. Therefore the wavelengths at which the filter starts to act are not as close to the most energetic wavelengths as the numbers given above might lead one to believe. Thus, the energetic scales are well resolved in most of our experiments, 
although resolution of scales containing most of the enstrophy, which is dominated by smaller scales, will not be as good.

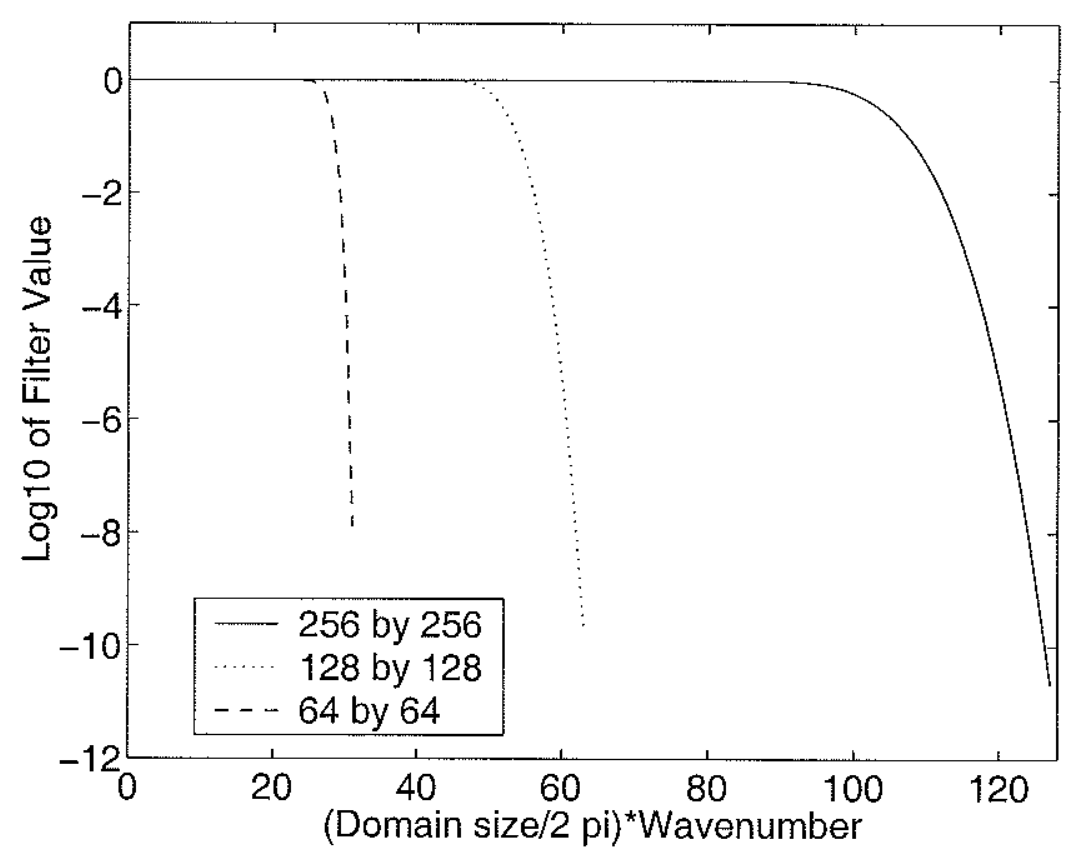

Figure 2-2: Exponential cut-off wavenumber filter used in 64 squared, 128 squared, and 256 squared experiments. The $\mathrm{x}$-axis is $\frac{\text { domain size }}{2 \pi}$ (10 in these experiments) times the model wavenumbers. A value of 64 corresponds to a wavelength equal to the deformation radius.

The major advantage of using a filter for small-scale dissipation is that larger scales are completely unaffected by the filter. Hyperviscosity, on the other hand, affects all scales to some extent. The repercussions of this are demonstrated in LaCasce (1996). There a barotropic dipolar vortex ("modon") was numerically time-stepped in a 128 by 128 domain using three different forms of small-scale dissipation: the exponential cut-off wavenumber filter, a Laplacian damping $\left(\nu \nabla^{2} q\right)$, and a hyperviscous damping $\left(\nu \nabla^{8} q\right)$. The vortex under the filter retained its shape and lost only one percent of its amplitude. Under both Laplacian and hyperviscous damping, however, the vortex suffered distortion as well as substantial loss of amplitide. The filter therefore yielded the response most consistent with inviscid dynamics.

One disadvantage of the filter is that its effects on energy and enstrophy dissipa- 
tion are not easily rendered in analytical form. The form of energy and enstrophy dissipation due to hyperviscosity can easily be found analytically, and computed explicitly in the numerical model. In this case, the analytical form would involve the squares of high derivatives of the streamfunction and would thus have no simple physical explanation. An alternative approach, adopted in this work, is to calculate the effects of small-scale dissipation on energy and enstrophy balances as residuals.

Because cutoff wavelength moves to increasingly smaller scales as resolution increases, solutions obtained with a wavenumber filter may change with resolution. Most of the results in this dissertation displayed relatively small (nominally, less than thirty percent) changes in quantitities such as energy levels as resolution increased. But there is one class of solutions-the vortex regime discussed in chapter 6-for which energy increases dramatically with increasing resolution, so much so that equilibration was not obtained after several weeks of computer integration time. Very similar vortex solutions exist under the action of hyperviscosity, but at energies low enough to reach equilibrium in one week or so of processor time. We therefore adopt hyperviscosity as our choice of small-scale dissipation in the vortex regime. With a fixed coefficient $\nu$ and exponent $n$ of hyperviscosity, energy levels are little affected when resolution increases from 128 squared to 256 squared grid points. This is because with fixed $\nu$ and $n$, the finer scales introduced with higher resolution are heavily damped.

\subsubsection{Implementation of hyperviscosity}

In the presence of hyperviscosity, the quasi-geostrophic equation that must be solved in the spectral model is:

$$
\frac{\partial}{\partial t} \widehat{q_{N}}=O T-\nu k^{2 n} \widehat{q_{N}}
$$

where OT stands for other terms in the equation and $\mathrm{N}$ takes on the value 1 or 2 
depending on the layer in question. For simplicity, we drop $\mathrm{N}$ in what follows, and write $q$ in place of $\hat{q}$.

Direct evaluation of equation 2.36 is difficult, because $k^{2 n}$ becomes quite large for the largest wavenumbers. As a result, small timesteps are needed to avoid violating the "c.f.l. condition" for numerical stability, and the experiment becomes too computationally expensive to be feasible. To circumvent this, a "semi-implicit" scheme is widely used in the discretization of 2.36 :

$$
q(t+\Delta t)-q(t)=\Delta t O T-\Delta t \nu k^{2 n}\left(\frac{q(t+\Delta t)+q(t)}{2}\right)
$$

Solving for $q(t+\Delta t)$ yields:

$$
q(t+\Delta t)=\frac{\Delta t O T}{1+\frac{\Delta t \nu k^{2 n}}{2}}+\left(\frac{1-\frac{\Delta t \nu k^{2 n}}{2}}{1+\frac{\Delta t \nu k^{2 n}}{2}}\right) q(t)
$$

Unless $\Delta t$ is very small, as $k \rightarrow \infty$, the coefficient of $q(t)$ in equation 2.38 asymptotes to -1 . Since one is not a large number, numerical integration with coarser timesteps is possible. Consider, however, the behavior of 2.36 in the absence of OT. The solution then is:

$$
q_{N}=q_{0} e^{-\nu k^{2 n} t}
$$

where $q_{0}$ is a constant, meaning that:

$$
q(t+\Delta t)=e^{-\nu k^{2 n} \Delta t} q(t)
$$

Therefore, for large $k$ and finite $\Delta t$, the coefficient of $q(t)$ asymptotes to zero, not one as in the semi-implicit scheme. Thus, while the semi-implicit scheme for hyperviscosity is numerically stable for large timesteps, it is not a correct implementation of 2.36 . 
Glenn Flierl (personal communication) has invented an alternative scheme for incorporating hyperviscosity into the qgb numerical model. It begins with the following equation, which is easily shown to be equivalent to equation 2.36 :

$$
\frac{\partial}{\partial t}\left(e^{\nu k^{2 n} t} q\right)=e^{\nu k^{2 n} t} O T .
$$

In finite-difference form this becomes:

$$
e^{\nu k^{2 n}(t+\Delta t)} q(t+\Delta t)-e^{\nu k^{2 n} t} q(t)=\Delta t e^{\nu k^{2 n} t} O T .
$$

Dividing by $e^{\nu k^{2 n} t}$ gives:

$$
q(t+\Delta t)=e^{-\nu k^{2 n} \Delta t}(q(t)+\Delta t O T) .
$$

This scheme is implemented in the Adams-Bashforth timestepping scheme used by the qgb code as:

$$
\begin{gathered}
q(t+\Delta t)=e^{-\lambda \Delta t} \\
{\left[q(t)+d t 0 O T(t)+d t 1 O T(t-\Delta t) e^{-\lambda \Delta t}+d t 2 O T(t-2 \Delta t) e^{-2 \lambda \Delta t}\right]}
\end{gathered}
$$

where $\lambda=\nu k^{2 n}$, and dt0, dt1, and dt2 are fractions of $\Delta t$ dictated by the AdamsBashforth scheme:

$$
\begin{gathered}
d t 0=\frac{23}{12} \Delta t \\
d t 1=-\frac{16}{12} \Delta t
\end{gathered}
$$




$$
d t 2=\frac{5}{12} \Delta t
$$

As discussed above, this implementation of hyperviscosity is equivalent to the direct evaluation of 2.36, and is thus correct, unlike the semi-implicit scheme. However, the exponent on the right-hand side of equation 2.44 is negative, and thus the exponential does not become overly large for large wavenumbers. Therefore, unlike a direct evaluation scheme, our scheme does not require expensively small timesteps. In fact, it can suffer from the opposite problem; for large $k$, the exponential may become so small that the computer cannot distinguish the values from zero. This is especially true since the Adams-Bashforth timestepping scheme requires the square and cube of the exponential. Therefore, where this hyperviscosity scheme is used, matrices of the exponential operator along with its square and cube are checked to ensure that they do not contain an inordinate number of zeroes.

\subsection{Linear stability analysis}

The main results of this thesis are strongly nonlinear numerical integrations, for which analytical interpretation is often difficult. Important insights can be gained from a linear stability analysis, which is analytically tractable (c.f. Pedlosky 1987 and references therein). If nonlinear Jacobian terms are dropped, and wavelike solutions of the form $e^{i(k x+l y-\omega t)}$ are assumed, the governing equations (equation 2.1 and equation 2.2) can be written like so:

$$
B \vec{q}+C \vec{\psi}=\omega \vec{q}
$$

where

$$
\vec{q}=\left(\begin{array}{l}
q_{1} \\
q_{2}
\end{array}\right)
$$




$$
\begin{gathered}
\vec{\psi}=\left(\begin{array}{c}
\psi_{1} \\
\psi_{2}
\end{array}\right), \\
B=\left(\begin{array}{cc}
\overline{u_{1}} k+\overline{v_{1}} l & 0 \\
0 & \overline{u_{2}} k+\overline{v_{2}} l
\end{array}\right),
\end{gathered}
$$

and

$$
C=\left(\begin{array}{cc}
k \frac{\partial \overline{q_{1}}}{\partial y}-l \frac{\partial \overline{q_{1}}}{\partial x}+i R_{1}\left(k^{2}+l^{2}\right) & 0 \\
0 & k \frac{\partial \overline{q_{2}}}{\partial y}-l \frac{\partial \overline{q_{2}}}{\partial x}+i R_{2}\left(k^{2}+l^{2}\right)
\end{array}\right),
$$

where mean PV gradients are given by equations 2.8 through 2.11 . The frequency $\omega$ can be complex; the presence of imaginary parts implies exponential growth or decay, depending on sign. Linear stability analysis can predict the length scales of fluctuations which grow the fastest, and therefore often dominate the flow.

The inversion relationship between the streamfunctions and potential vorticities can also be written in matrix form:

$$
\vec{\psi}=A^{-1} \vec{q},
$$

where

$$
A=\left(\begin{array}{cc}
-\left(k^{2}+l^{2}\right)-\frac{1}{R_{d}^{2}(1+\delta)} & \frac{1}{R_{d}^{2}(1+\delta)} \\
\frac{\delta}{R_{d}^{2}(1+\delta)} & -\left(k^{2}+l^{2}\right)-\frac{\delta}{R_{d}^{2}(1+\delta)}
\end{array}\right)
$$

which can be inverted to give:

$$
A^{-1}=\frac{R_{d}^{2}}{\left(k^{2}+l^{2}\right)\left[1+\left(k^{2}+l^{2}\right) R_{d}^{2}\right]}\left(\begin{array}{cc}
-\left(k^{2}+l^{2}\right)-\frac{\delta}{R_{d}^{2}(1+\delta)} & -\frac{1}{R_{d}^{2}(1+\delta)} \\
-\frac{\delta}{R_{d}^{2}(1+\delta)} & -\left(k^{2}+l^{2}\right)-\frac{1}{R_{d}^{2}(1+\delta)}
\end{array}\right) .
$$


Combining 2.51 and 2.46 yields the eigenvalue problem:

$$
\left(B+C A^{-1}\right) \vec{q}=\omega \vec{q}
$$

In this dissertation, equation 2.54 is solved using a linear stability code written in MATLAB. Given input parameters $\overline{u_{1}}, \overline{u_{2}}, \overline{v_{1}}, \overline{v_{2}}, \beta, \delta, R_{1}, R_{2}$, and $R_{d}$, the linear analysis code calculates growth rates for wavenumbers $k$ and $l$, where $k$ and $l$ take on both positive and negative values and range in magnitude from $\frac{1}{1000} \bullet \frac{2 \pi}{R_{d}}$ to $1000 \bullet \frac{2 \pi}{R_{d}}$.

The linear stability code proved useful at several junctures in the course of this research. For example, several tests of the qgb model were performed by assigning specific values of the background parameters given above and initializing it with small-amplitude monochromatic waves of specific wavenumbers $k$ and $l$. Growth rates calculated from the qgb model matched those calculated from 2.54 to within five percent for all tests performed. More uses of the linear stability code in the dissertation will be described where appropriate. 


\section{Chapter 3}

\section{Effects of stratification and}

\section{friction on eddy baroclinicity and}

\section{length scales}

\subsection{Inverse cascades in two-dimensional flows}

In a pioneering effort, Fjortoft (1953) demonstrated that conservation of squared relative vorticity (enstrophy) as well as energy in two-dimensional turbulence leads to the so-called inverse cascade of energy to larger scales. Over time, the argument for an inverse cascade has been refined. What follows is taken from Salmon (1998), but similar arguments can be found in many other sources.

Consider purely two-dimensional motions, governed by the equation:

$$
\frac{\partial q}{\partial t}+J(\psi, q)=0
$$

where $q=\nabla^{2} \psi$. If one multiplies this equation by $\psi$ and $q$, and integrates over a domain assumed to be doubly periodic, there are no contributions from the second term on the left-hand side, and we obtain the energy and enstrophy conservation 
equations, which we write in both physical and spectral space:

$$
\begin{gathered}
\frac{\partial}{\partial t} \iint(\nabla \psi)^{2} d x d y=\frac{\partial}{\partial t} \int E(k) d k=0, \\
\frac{\partial}{\partial t} \iint\left(\nabla^{2} \psi\right)^{2} d x d y=\frac{\partial}{\partial t} \int k^{2} E(k) d k=0,
\end{gathered}
$$

where $E(k)$ is the wavenumber spectrum of kinetic energy. We now assume that energy initially concentrated at wavenumber $k_{1}$ spreads to other wavenumbers over time, so that:

$$
\frac{\partial}{\partial t} \int\left(k-k_{1}\right)^{2} E(k) d k \geq 0
$$

But

$$
\frac{\partial}{\partial t} \int\left(k-k_{1}\right)^{2} E(k) d k=-2 k_{1} \frac{\partial}{\partial t} \int k E(k) d k
$$

because of the conservation of energy and enstrophy. Therefore

$$
\frac{\partial}{\partial t} k_{E} \leq 0
$$

where $k_{E}$, the first moment of the energy spectrum (often called the centroid), is defined by:

$$
k_{E}=\frac{\int k E(k) d k}{\int E(k) d k}
$$

Energy therefore cascades to larger scales in two-dimensional turbulence. On the other hand, similar reasoning (c.f. Salmon 1998) implies that moments of the enstrophy spectrum cascade to smaller scales over time. We demonstrate these arguments 
with a numerical experiment, in which equation 3.1 (with a wavenumber cut-off filter added to the right hand side for numerical stability) is integrated in a 256 by 256 domain. Figure 3-1 shows the initial and final streamfunction and vorticity fields after a long time integration. The streamfunction has indeed cascaded to larger scales, while compact coherent vortices are visible in the PV field (McWilliams 1984). Only three percent of the initial energy was lost in this integration, while 94 percent of the enstrophy was consumed by the wavenumber filter. This is because enstrophy cascades to small scales while energy cascades to larger scales, beyond the reach of the filter. A philosophical digression: many articles and textbooks utilize conservation of enstrophy in arguing for the existence of an inverse cascade of energy, and the author will continue to do so as well. As a practical matter, however, the downscale transfer of enstrophy means that it will be dissipated, either by reaching true dissipation scales in an actual fluid, or by reaching the smallest resolved scales in a numerical fluid. Enstrophy is therefore not really conserved, but the argument for a cascade can be modified to allow for this. If we acknowledge dissipation of enstrophy:

$$
\frac{\partial}{\partial t} \int k^{2} E(k) d k \leq 0
$$

then equation 3.4 will imply that:

$$
-2 k_{1} \frac{\partial}{\partial t} \int k E(k) d k \geq-\frac{\partial}{\partial t} \int k^{2} E(k) d k \geq 0
$$

and the inverse cascade still holds. See Batchelor (1969) and Bretherton and Haidvogel (1976) for a more detailed discussion. The cascade arguments to be presented throughout the dissertation can be altered in like manner to include enstrophy dissipation but we omit this for the sake of brevity. 
Initial Psi

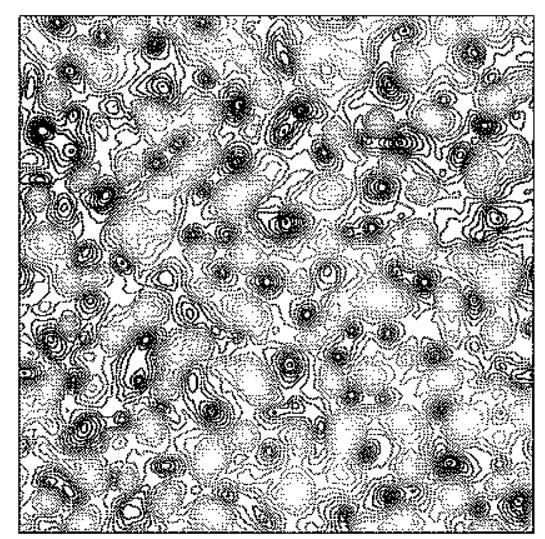

Final Psi

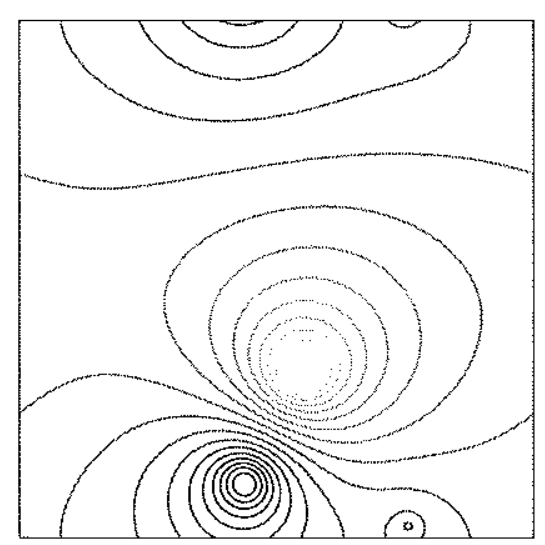

Initial q

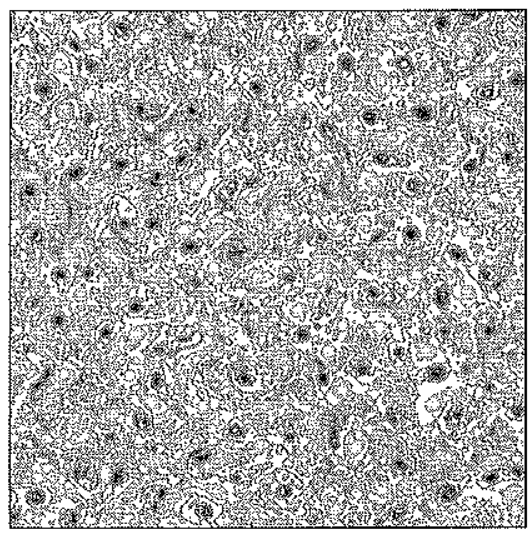

Final q

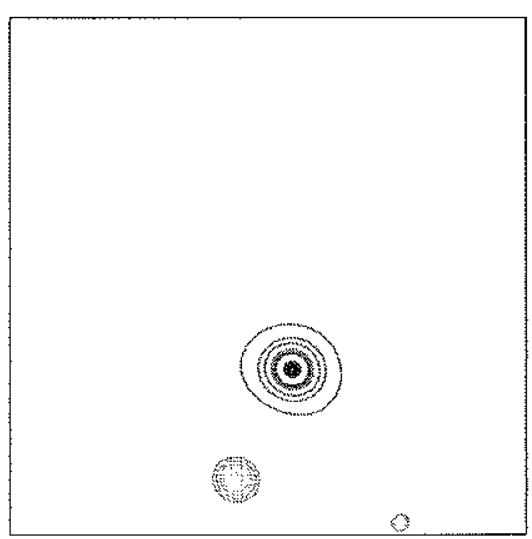

Figure 3-1: An example of the inverse cascade in freely evolving two-dimensional turbulence. 


\subsection{Barotropization and inverse cascades in strat- ified QG flows}

Stratified quasi-geostrophic flows are not purely two-dimensional because stratification introduces the possibility of baroclinic (depth-dependent) motions. Charney (1971) argued that potential enstrophy $q^{2}$ in stratified quasi-geostrophy is analagous to enstrophy in two-dimensional motion, so that an inverse cascade should still take place. In the stratified case, the inverse cascade includes energy transfer to larger vertical scales (i.e. the barotropic mode) as well as larger horizontal scales. Rhines (1977) demonstrated barotropization and the inverse cascade with numerical experiments and complementary theoretical arguments. Below the author presents his own version of these arguments and an illustrative example. In the absence of forcing and dissipation, the modal energy and enstrophy equations 2.29 and 2.30 are:

$$
\begin{gathered}
\frac{\partial}{\partial t} \iint \frac{1}{2}\left[\left(\nabla \psi_{B T}\right)^{2}+\left(\nabla \psi_{B C}\right)^{2}+\frac{\psi_{B C}^{2}}{R_{d}^{2}}\right] d x d y=0 \\
\frac{\partial}{\partial t} \iint \frac{1}{2}\left[\left(\nabla^{2} \psi_{B T}\right)^{2}+\left(\nabla^{2} \psi_{B C}\right)^{2}+\frac{1}{R_{d}^{2}}\left(\nabla \psi_{B C}\right)^{2}+\frac{1}{R_{d}^{2}}\left(\left(\nabla \psi_{B C}\right)^{2}+\frac{\psi_{B C}^{2}}{R_{d}^{2}}\right)\right] d x d y=0 .
\end{gathered}
$$

Now write the barotropic and total baroclinic energies in spectral form:

$$
\begin{gathered}
\iint \frac{1}{2}\left(\nabla \psi_{B T}\right)^{2} d x d y=\int E_{B T}(k) d k \\
\left.\iint \frac{1}{2}\left[\left(\nabla \psi_{B C}\right)^{2}+\frac{1}{R_{d}^{2}} \psi_{B C}^{2}\right)\right] d x d y=\int E_{B C}(k) d k .
\end{gathered}
$$

Then the energy and enstrophy conservation statements can be written: 


$$
\begin{gathered}
\frac{\partial}{\partial t} \int\left[E_{B T}(k)+E_{B C}(k)\right] d k=0 \\
\frac{\partial}{\partial t} \int\left[k^{2} E_{B T}(k)+\left(k^{2}+\frac{1}{R_{d}^{2}}\right) E_{B C}(k)\right] d k=0
\end{gathered}
$$

Let us define centroids of the barotropic and total baroclinic energy spectra:

$$
\begin{aligned}
& k_{B T}=\frac{\int k E_{B T}(k) d k}{\int E_{B T}(k) d k}, \\
& k_{B C}=\frac{\int k E_{B C}(k) d k}{\int E_{B C}(k) d k},
\end{aligned}
$$

and then assume a spreading of energy in both modal spectra, so that addition gives:

$$
\frac{\partial}{\partial t} \int\left[\left(k-k_{B T}\right)^{2} E_{B T}(k)+\left(k-k_{B C}\right)^{2} E_{B C}(k)\right] d k \geq 0,
$$

which leads to:

$$
\frac{\partial}{\partial t} \int\left[k^{2}\left(E_{B T}(k)+E_{B C}(k)\right)-k_{B T}^{2} E_{B T}(k)-k_{B C}^{2} E_{B C}(k)\right] d k \geq 0 .
$$

Using equation 3.15 , we substitute for the first two terms in 3.19 to obtain:

$$
\frac{\partial}{\partial t} \int\left[\frac{1}{R_{d}^{2}} E_{B C}(k)+k_{B T}^{2} E_{B T}(k)+k_{B C}^{2} E_{B C}(k)\right] d k \leq 0 .
$$

Conservation of total energy allows this to be rewritten as:

$$
\frac{\partial}{\partial t}\left[k_{B T}^{2} E+\left(\frac{1}{R_{d}^{2}}+k_{B C}^{2}-k_{B T}^{2}\right) \int E_{B C}(k) d k\right] \leq 0,
$$


where $\mathrm{E}$ is the total energy, thus a constant. This inequality suggests that the cascade acts to reduce the quantity in brackets on the left hand side. Thus the length scale of the barotropic mode should increase, while total baroclinic energy decreases. Since $R_{d}$ is fixed, it does little good, from the point of view of reducing the second term in brackets, to lower the baroclinic wavenumber; instead, decreasing total baroclinic energy is more effective. In summary, therefore, we expect the fluid to become more barotropic, and to increase its barotropic length scale, but for the baroclinic length scale to be little affected. Rhines (1977) has pointed out that the process can also be interpreted as a release of potential energy into kinetic energy, somewhat akin to baroclinic instability. Potential energy release flattens isopycnals, leading to a barotropic final state.

Slightly different information can be obtained by following the example of Salmon (1998) and writing the spreading of the total energy spectrum around an initial concentration at wavenumber $k_{1}$ as follows:

$$
\frac{\partial}{\partial t} \int\left(k-k_{1}\right)^{2}\left(E_{B T}(k)+E_{B C}(k)\right) d k \geq 0
$$

which implies that:

$$
\frac{\partial}{\partial t}\left[2 k_{1} \int k\left(E_{B T}(k)+E_{B C}(k)\right) d k+\frac{1}{R_{d}^{2}} \int E_{B C}(k) d k\right] \leq 0 .
$$

The latter equation suggests once again that total baroclinic energy should decrease over time, as well as that the length scale associated with total energy should increase.

The inverse cascade to larger horizontal scales and to the barotropic mode is evident in figure 3-2, which contours the evolution of a freely evolving two-layer $\delta$ $=1$ experiment in a 256 squared domain. More details of this run will be discussed in section 3.4. The initial condition is taken from one of the equilibrated forceddissipated solutions to be discussed later in this chapter. 


\section{Initial Psi1, H1 = H2 Run}

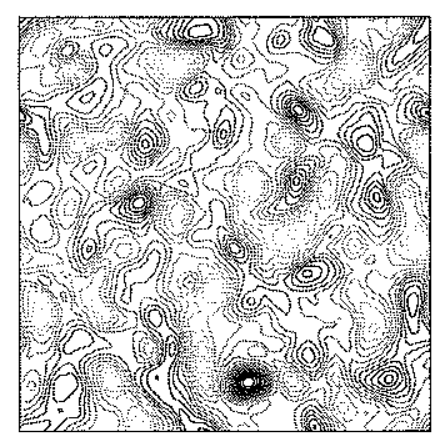

Final Psi1

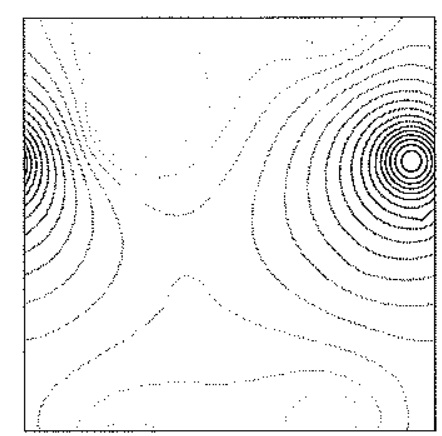

Initial Psi2

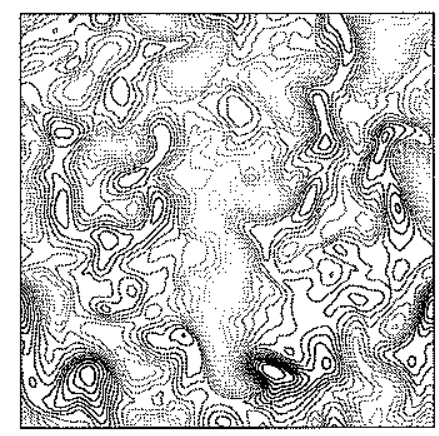

Final Psi2

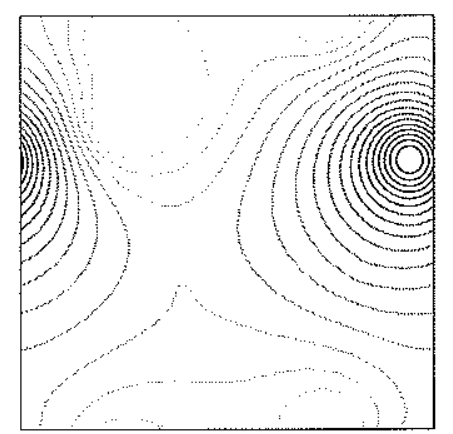

Figure 3-2: An example of barotropization (as indicated by the greater degree of correlation between layers in the final state) and the inverse cascade in freely evolving two-layer turbulence with equal layer depths. 


\subsection{Can the level of barotropization be reduced with surface-trapped stratification and bottom- trapped friction?}

Two-layer flat-bottom quasi-geostrophic turbulence demonstrably cascades to the barotropic mode and to larger length scales. Here we argue that the cascade may weaken in models with a surface-trapped stratification, for two reasons. The first mechanism we present is relevant to all of the cases examined in this chapter, while the second is relevant when Ekman friction is bottom-trapped.

In a two-layer model with layer depths $H_{1}$ and $H_{2}$, the barotropic (BT) and baroclinic (BC) modes are given by:

$$
\begin{gathered}
F_{B T}=\left(\begin{array}{l}
1 \\
1
\end{array}\right) \\
F_{B C}=\left(\begin{array}{c}
\sqrt{\frac{H_{2}}{H_{1}}} \\
-\sqrt{\frac{H_{1}}{H_{2}}}
\end{array}\right)
\end{gathered}
$$

where the two elements in each column vector represent the values of the structure function in the top and bottom layers, respectively.

Nonlinear interaction between two BC modes is represented by the square of $\mathrm{F}_{B C}$, which projects onto the $\mathrm{BT}$ and $\mathrm{BC}$ modes as follows:

$$
F_{B C}^{2}=a * F_{B T}+b * F_{B C} .
$$

which yields:

$$
a=1
$$




$$
b=\frac{H_{2}-H_{1}}{\sqrt{H_{1} * H_{2}}}
$$

Note that $b$ equals zero if and only if the two layer depths are equal. In this case, nonlinear interactions between BC modes project only onto the BT mode. When layer depths are unequal, energy injected into the BC mode via the instability process has a stronger chance of remaining there. This can be seen directly in the modal forms of the governing PV equations 2.18 and 2.19. Nonlinear baroclinicbaroclinic interactions always project onto the barotropic mode, through the term $J\left(\psi_{B C}, \nabla^{2} \psi_{B C}\right)$. In the baroclinic equation, however, this Jacobian is multiplied by a factor of $\frac{1-\delta}{\sqrt{\delta}}$, meaning that nonlinear BC-BC interactions project onto the baroclinic mode if and only if layer depths are unequal. Therefore barotropization ought to be reduced when $\delta$ is not equal to one. This dissertation will compare eddy baroclinicity in numerical experiments having equal layer depths to those in experiments having unequal layer depths. In the unequal layer depth experiments it would be desirable to have a layer depth ratio $\delta=\frac{H_{1}}{H_{2}}$ representative of mid-ocean conditions. Flierl (1978) and Fu and Flierl (1980) chose a typical mid-ocean vertical profile of buoyancy frequency, calculated the associated baroclinic modes, and then calculated the projection of the square of the first baroclinic mode onto itself, in other words, the continuous analogue of equation 3.26. They found that a $\delta$ value of 0.2 yields the same self-projection coefficient ( $b$ in equation 3.26 ) as calculated from the actual ocean profile. Note that this is approximately the ratio of the depth of the thermocline to that of the abyss in a typical mid-ocean location.

In this chapter, the effect of $\delta$ on eddy baroclinicity will be tested first in freely evolving experiments, and then in sets of forced-dissipated experiments. All experiments are on an f-plane. The Fu and Flierl (1980) nonlinear self-projection idea has been tested only a few times before in geostrophic turbulence studies. Hua and Haidvogel (1986) tested the effects of nonuniform stratification in a few six-layer experiments and found that indeed the non-uniform $N^{2}$ cases retained more baro- 
clinicity. Treguier and Hua (1987) also examined the effects of nonuniform mean stratification on the eddy baroclinicity in their stochastic wind forcing numerical experiments. A more recent result is that of Smith and Vallis (2000), who studied freely evolving turbulence in a multi-layer model. Their vertical resolution permits them to examine energy transfer from higher baroclinic modes to the first baroclinic mode as predicted by Fu and Flierl (1980), as well as effects of small scale structures in the velocity profile. Neither of these effects can be studied in the two-layer models used in this dissertation. On the other hand, it is more computationally feasible to study a wide range of parameter space in a two-layer model. Here we perform a number of runs with symmetric Ekman friction-that is, Ekman friction which is equal in the two layers-as well as the bottom friction used in Hua and Haidvogel (1986) and in most other studies. In the latter case, stratification enhances baroclinicity for another reason besides the differences in nonlinear modal interactions. The barotropic mode is damped less strongly when the upper layer is thin and the Ekman friction occurs at the bottom only. As far as the author knows, this dissertation is the first occasion on which this frictional effect has been examined in the context of highly nonlinear baroclinic instability.

We will refer to $\delta=0.2$ experiments as having surface-trapped stratification, and to $\delta=1$ experiments as having uniform stratification. Since, in a two-layer model, the change in stratification occurs at the interface, these descriptions are not literally true. However, the $\delta=0.2$ (1) conditions are the best representatives in two-layer models of surface-trapped (uniform) stratification in the sense that the baroclinic mode is intensified (not intensified) at the surface, analogous to the surface-trapped (uniform) cases with continuous stratification. Then the nonlinear projections of the baroclinic mode onto itself, as well as the relative strengths of modal damping, are analogous as well.

Eddy interactions with bottom topography have also been proposed as a mechanism for halting barotropization and the inverse cascade-c.f. Rhines (1977) and 
Treguier and Hua (1988). This dissertation will not focus on topographic effects, but will discuss them briefly in later chapters.

\subsection{Effects of stratification on freely-evolving ex- periments}

Figure 3-3 displays the initial and final distributions of $\psi_{1}$ and $\psi_{2}$ for a freely evolving experiment with $\delta=0.2$. The initial modal streamfunctions, $\psi_{B C}$ and $\psi_{B T}$, are identical to those in the $\delta=1$ freely evolving experiment shown in figure 3-2. Initial modal energies are therefore equal, but the initial layer streamfunctions are different in the two cases. Barotropization and the inverse cascade are still visibly present, but quantitative measures will distinguish this experiment from its $\delta=1$ counterpart.

In both experiments, total eddy energy is conserved to within two percent over the length of the run. The top half of figure 3-4 confirms that the ratio of total baroclinic energy to barotropic energy decreases over time for both values of $\delta$. However, the offset between the experiments indicates that surface-trapped stratification does indeed retain more baroclinic energy. Thus these numerical experiments confirm the picture of modal interactions anticipated by Fu and Flierl (1980). Ratios of baroclinic to barotropic kinetic energies (not shown) behave similarly.

Eddy baroclinicity can also be measured via modal partition of enstrophies, although this must be done with caution since, as in the one-layer case, most of the initial enstrophy is dissipated. Modal enstrophy partitions are plotted in the bottom panel of figure 3-4. Transfer to the barotropic mode over time is still present, but to a lesser degree than in the modal energy partition. Again, offsets between experiments indicate that surface-trapped stratification retains more baroclinicity.

Length scales of the freely evolving experiments are plotted in figure 3-5. They were calculated as reciprocals of centroids of barotropic and total baroclinic energy spectra. The length scale associated with total energy is also plotted. As predicted, 


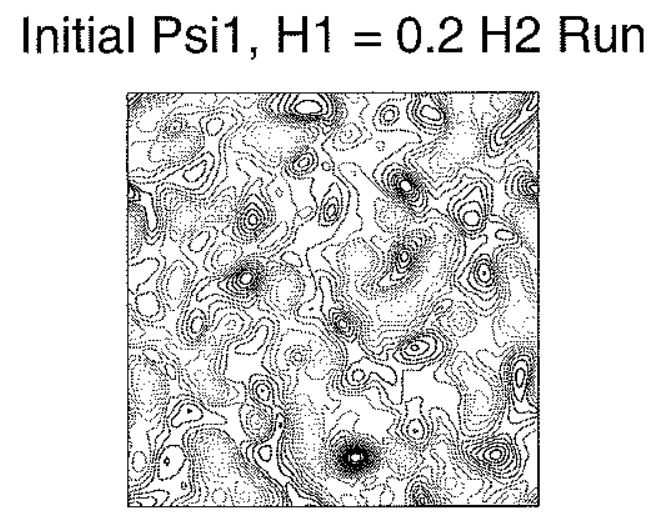

Final Psi1

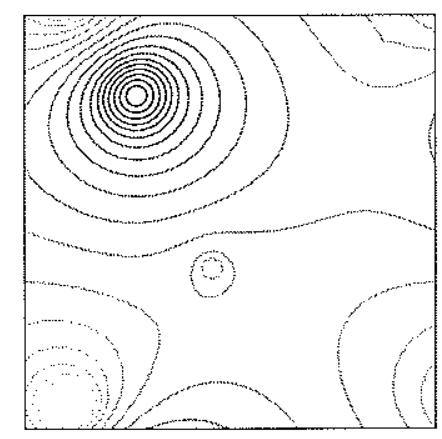

Initial Psi2

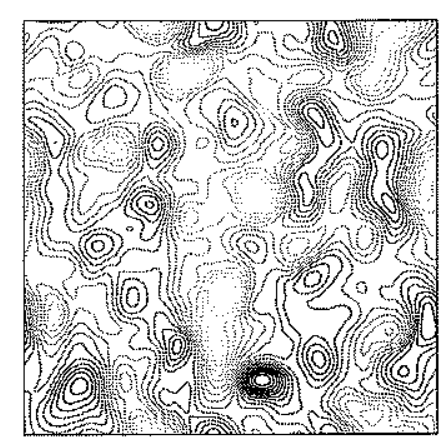

Final Psi2

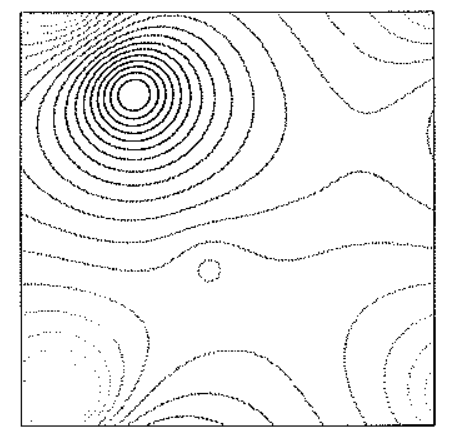

Figure 3-3: An example of barotropization and the inverse cascade in freely evolving two-layer turbulence with $\delta=0.2$. 

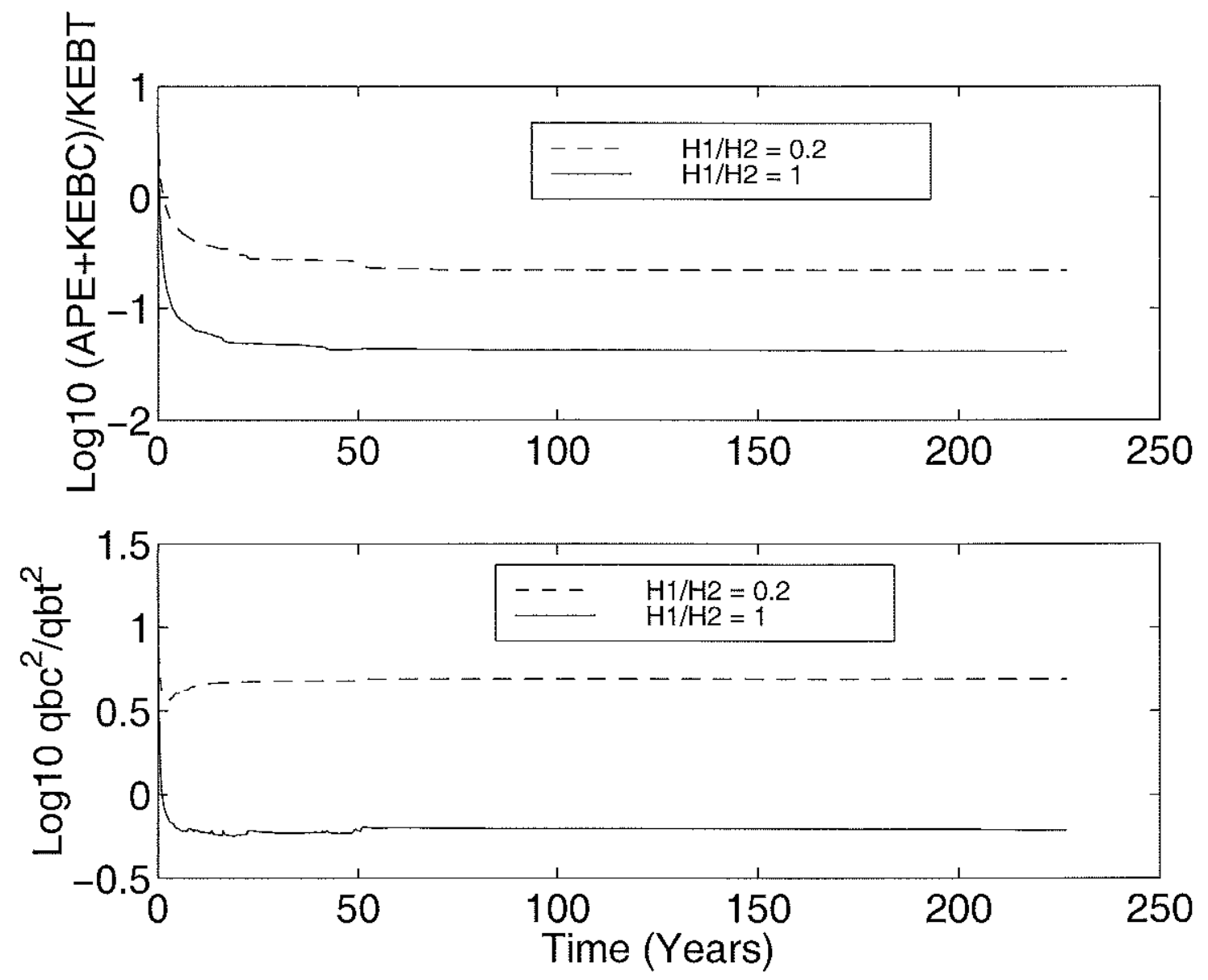

Figure 3-4: Ratios of modal energies (top panel) and enstrophies (bottom panel) in freely evolving experiments.

the total energy length scale has increased over time, as has the barotropic length scale. The increase in the baroclinic length scale is less dramatic, also as predicted. 


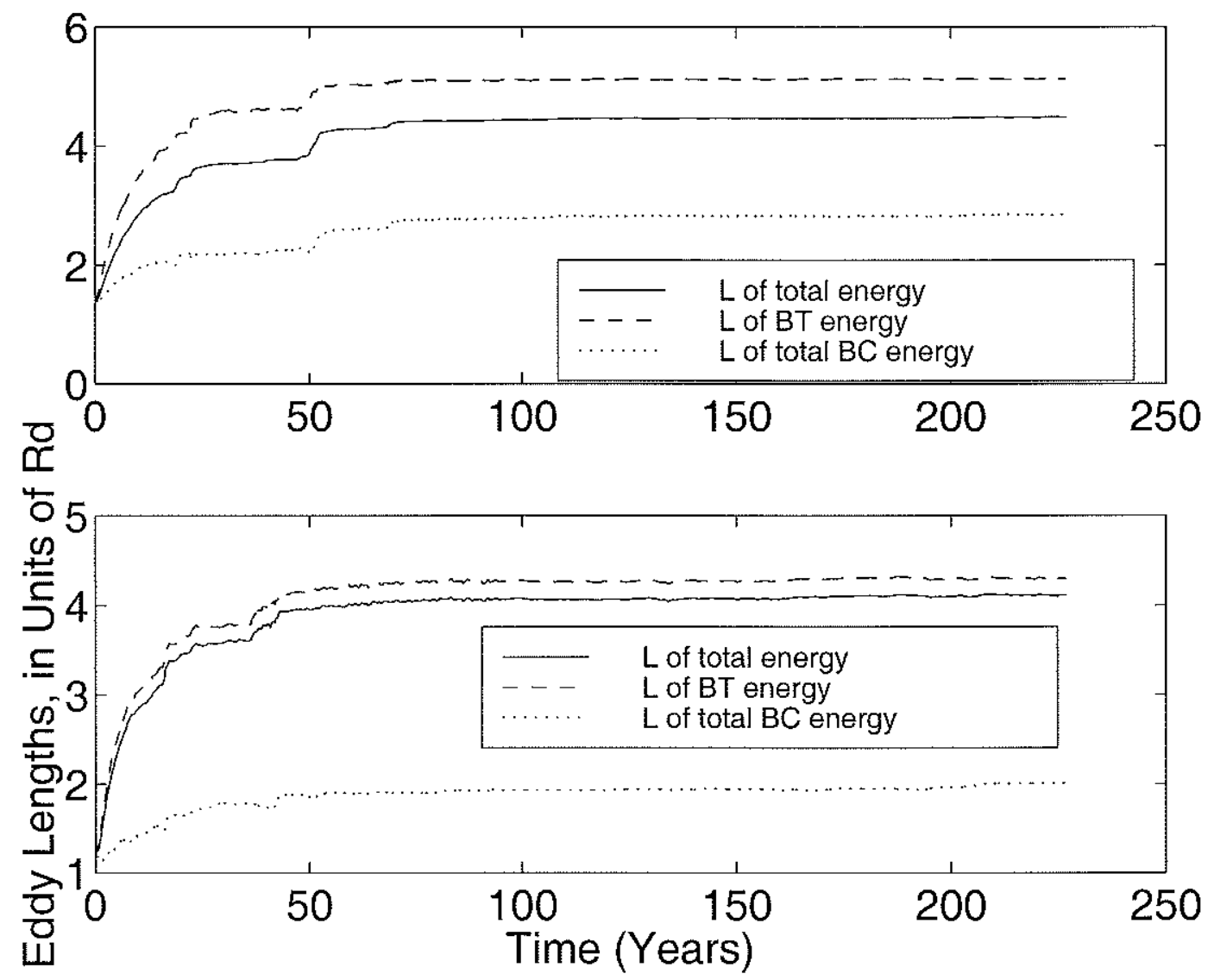

Figure 3-5: Eddy length scales in freely evolving $\delta=0.2$ (top panel) and $\delta=1$ (bottom panel) experiments.

\subsection{Baroclinicity in bottom-friction only forced- dissipated experiments}

In this section, we examine the baroclinicity of eddy fields forced with a zonal, vertically sheared flow on an f-plane and dissipated with bottom Ekman friction. Experiments are done with two values of $\delta$ to ascertain the effects of stratification on eddy baroclinicity.

First, we show an example spinup curve, figure 3-6. As with many of the dissertation results, this particular experiment was spun up at $64^{2}$ resolution, and a snapshot of the equilibrated low resolution solution was interpolated onto $256^{2}$ 
and then continued. Total eddy energy increased by about 25 percent in the $256^{2}$ run. Contours of upper layer fluctuation streamfunction during the spinup phase are displayed in figures 3-7 through 3-10. The initial condition, which was used to initialize many of the experiments in this thesis, was generated randomly (by grid point) in MATLAB. Note that increased resolution does not appear to change the qualitative nature of the turbulence. The spinup is nearly isotropic, with some hints of banded structures in the north-south direction (see for instance the 5.55 and 8.73 years plots). Near isotropy is consistent with linear growth rates, which are contoured as a function of zonal and meridional wavenumber in the top half of figure 3-11. Maximum growth rates are achieved when the meridional wavenumber $l=0$, consistent with the hint of north-south structures (note in all contour plots in this document east at the right, west is at the left, south is at the bottom, and north is at the top). Contours of linear growth rates for the inviscid case are shown in the same figure for comparison. The presence of bottom friction destabilizes large wavenumbers (small scales), consistent with arguments made in earlier studies (c.f. Williams and Robinson 1974). We will return to this fact later.

Experiments were performed with several different values of the bottom friction coefficient, to facilitate a range of model behaviors. For each experiment, plots of total eddy energy are examined in order to judge the time at which equilibration has been achieved; eddy statistics are taken as averages from that time forwards. Clearly, there is an unavoidable degree of subjectivity in deciding whether a particular run has equilibrated.

Total eddy energy and eddy kinetic energy are displayed as a function of the bottom friction coefficient in figure 3-12. Note that the $\mathrm{x}$ axis is $\log$ base ten of dissipation time, meaning that friction increases from right to left. Dissipation time varies from 0.7 days to 1451 days. Using the linear stability code, no value of bottom Ekman friction could be found that would stabilize the flow. Total eddy energy varies by about 2.5 orders of magnitude. At the less viscous end of the 


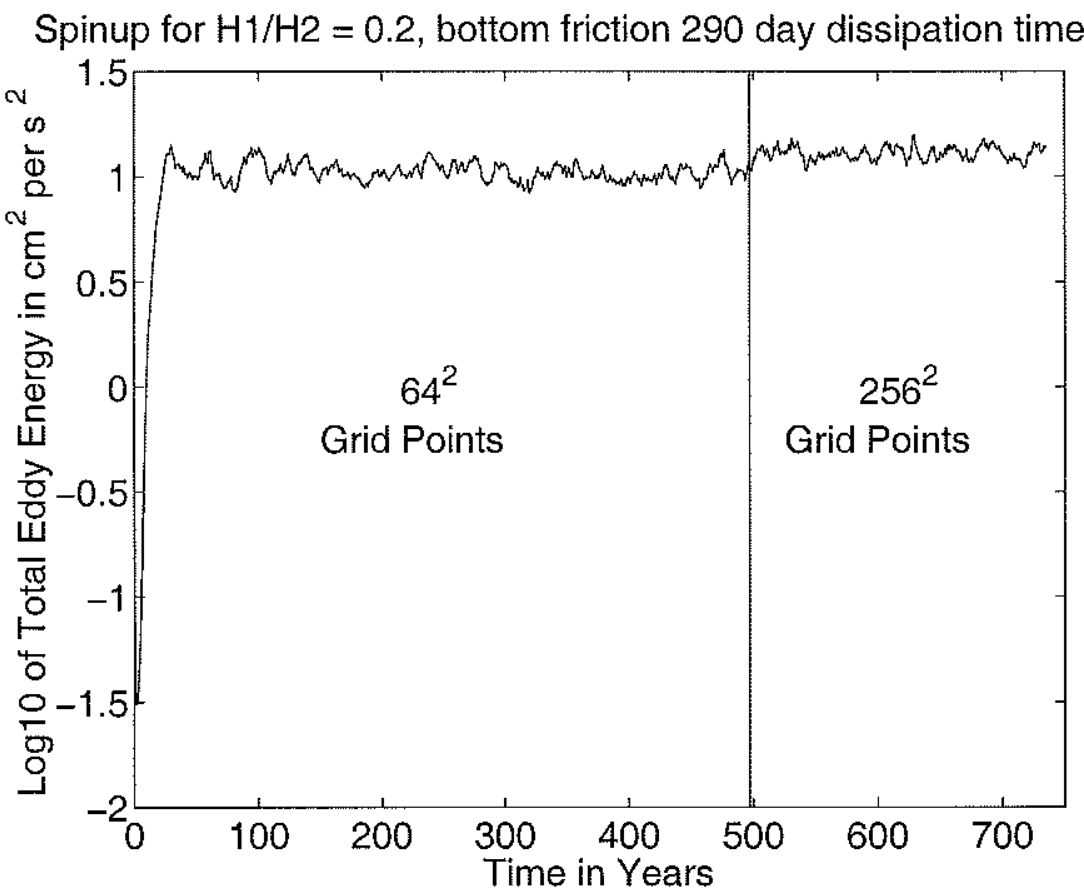

Figure 3-6: Spinup curve for the f-plane, zonal shear flow, $\delta=0.2,290$ day dissipation time bottom friction only experiment. The solid line demarcates the transition from $64^{2}$ to $256^{2}$ resolution.

experiments, energy increases monotonically as friction is reduced. At the viscous end, total energy increases with increasing friction, contrary to intuition. Nonmonotonicity is also present in kinetic energy, but is less pronounced, indicating that potential energy, which is unaffected by Ekman friction, is mainly responsible for the non-monotonic behavior of total energy with friction. It may be that large frictional values prohibit energy transfer to the lower layer, thus creating a buildup of potential energy. Linear growth rates (figure 3-13) continue to decrease as Ekman friction increases, so the non-monotonicity is apparently not explainable via unusual linear stability properties.

Estimates of frictional spin-down times in the ocean vary widely. Weatherly and Martin (1978) estimate a bottom boundary layer thickness of $10 \mathrm{~m}$; inserting this as an Ekman layer depth into 2.12, along with $f_{0}=10^{-4} \mathrm{~s}^{-1}$ and $H_{2}=4000 \mathrm{~m}$, yields a 100 day spin-down time for the barotropic mode. They note that earlier 


\section{Initial Psi1}

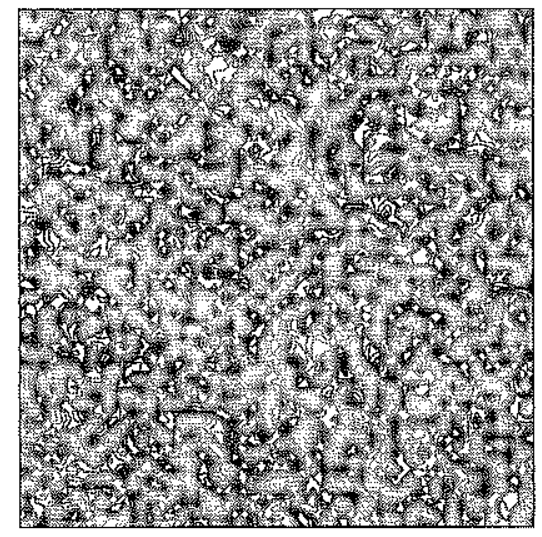

\subsection{Years}

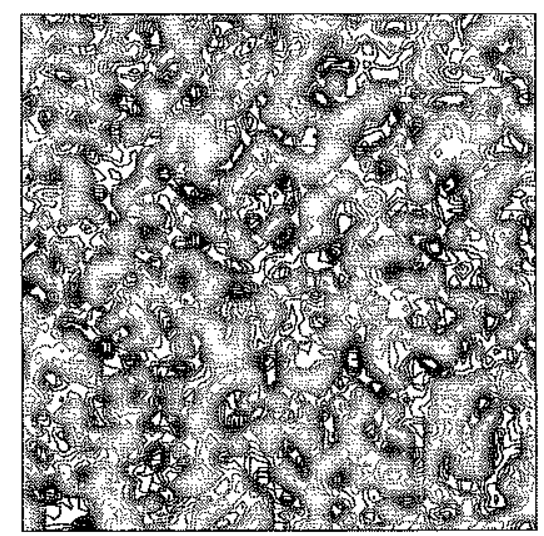

290 Days

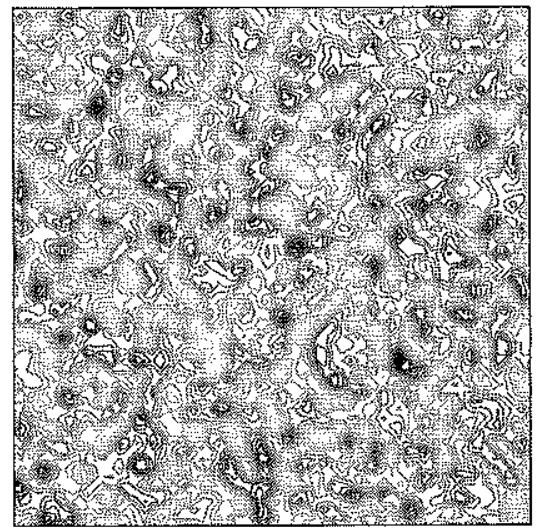

\subsection{Years}

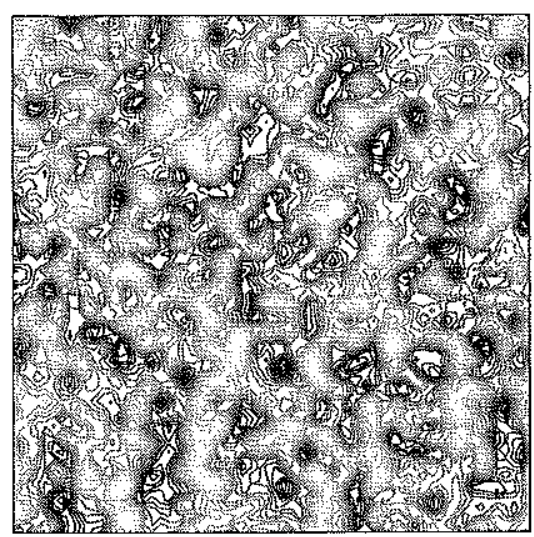

Figure 3-7: Contours of top layer fluctuation streamfunction for the spinup example, displayed at selected times. 


\subsection{Years}

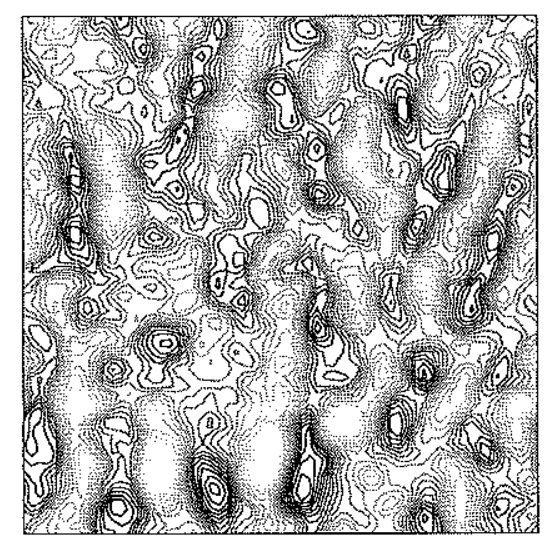

\subsection{Years}

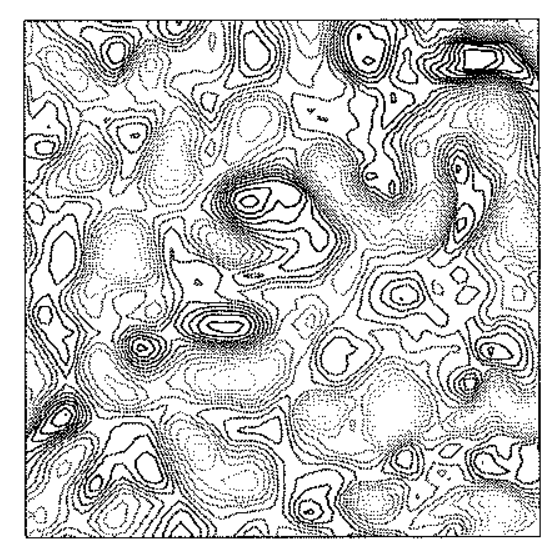

\subsection{Years}

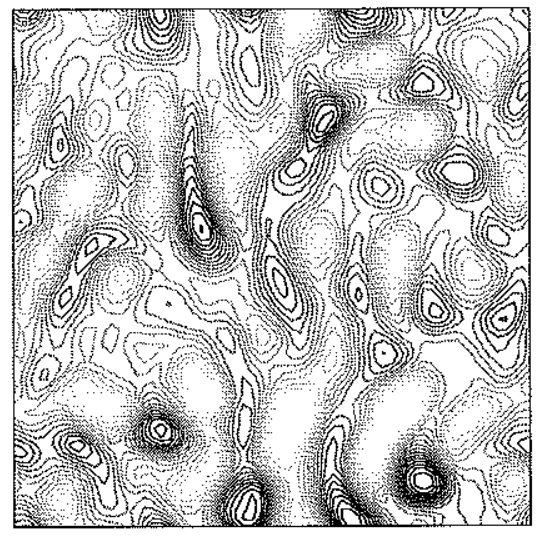

\subsection{Years}

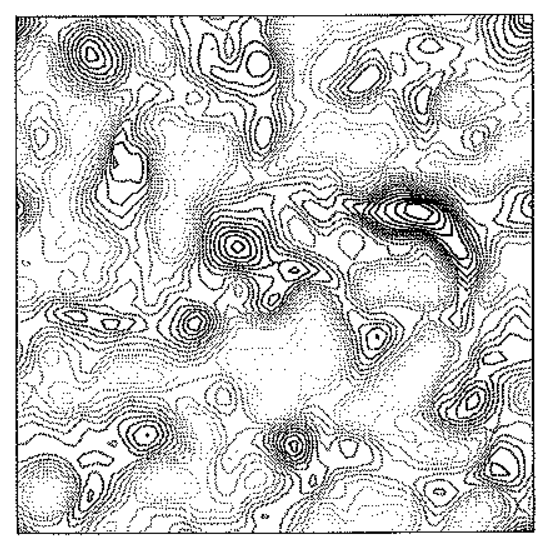

Figure 3-8: Contours of top layer fuctuation streamfunction for the spinup example, continued. 
18.27 Years

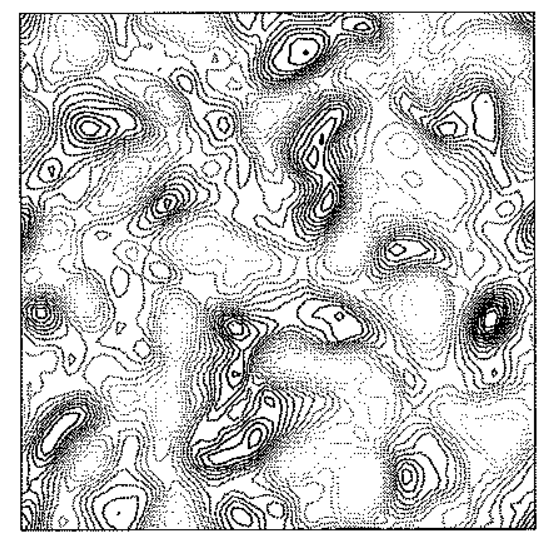

\subsection{Years}

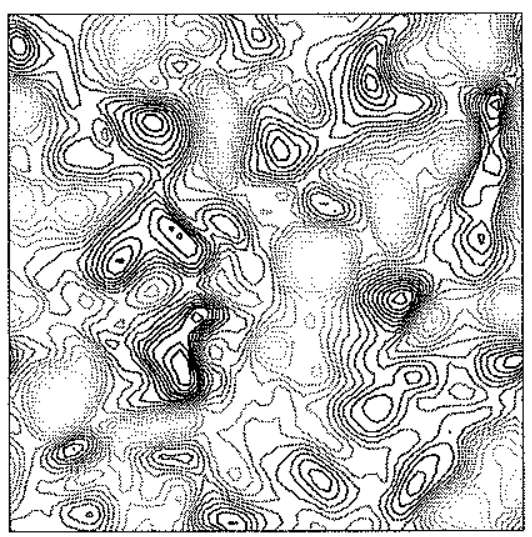

21.45 Years

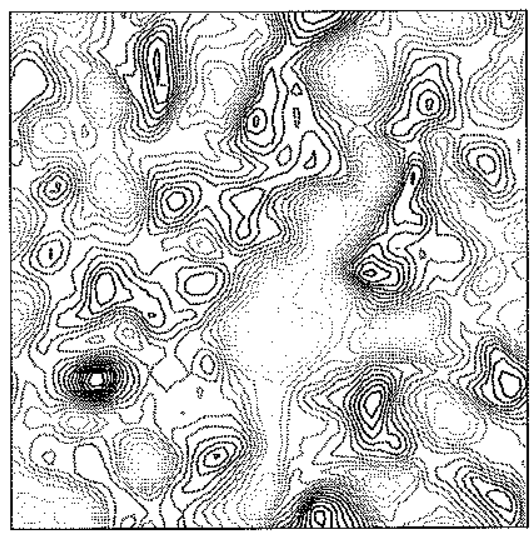

27.81 Years

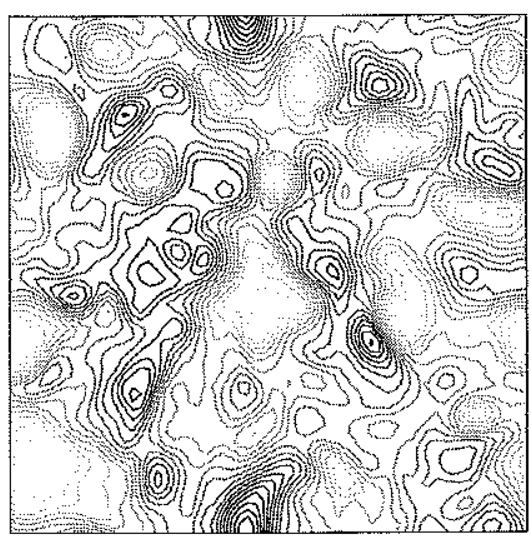

Figure 3-9: Contours of top layer fluctuation streamfunction for the spinup example, continued. By the last panels, the turbulence has reached equilibration. 


\section{Squared at Transition to 256 Squared}

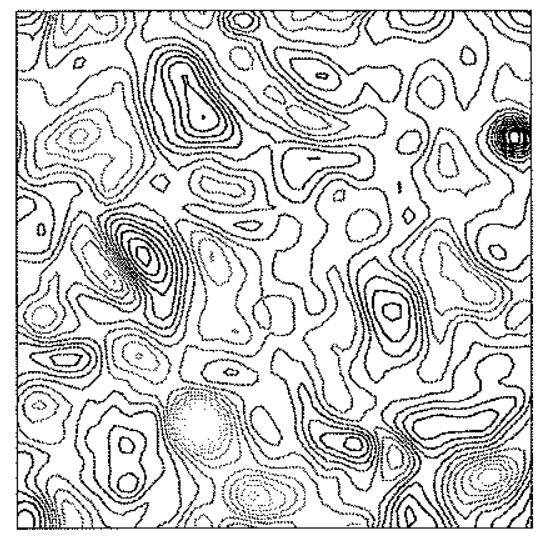

\section{End of 256 Squared Run}

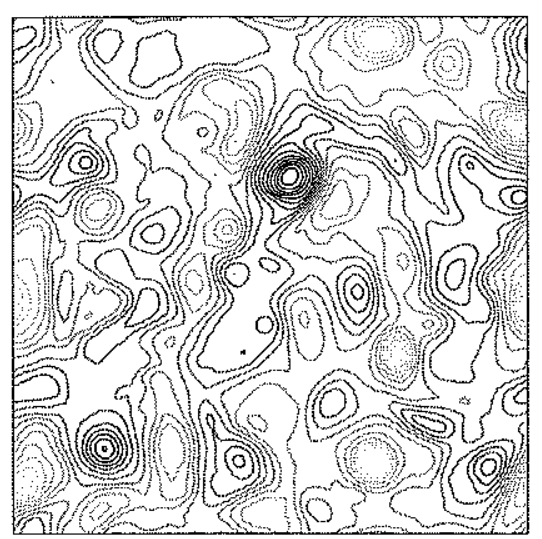

Figure 3-10: Effect of changing resolutions on top layer fluctuation streamfunction for the spinup example. 

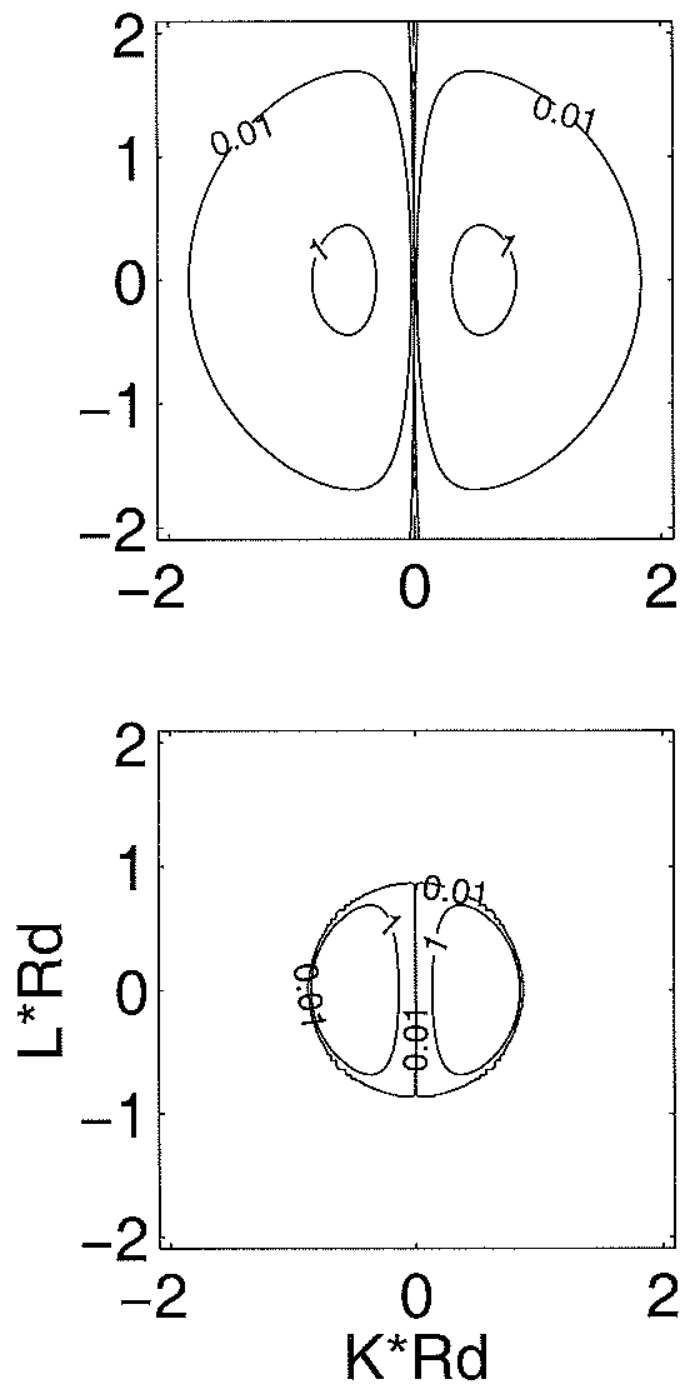

Figure 3-11: Top panel: Linear growth rates as functions of zonal and meridional wavenumbers $\mathrm{K}$ and $\mathrm{L}$, in units of $10^{-8} \mathrm{~s}^{-1}$, for the f-plane spinup example (with 290 day bottom Ekman friction). Bottom panel: Linear growth rates in the f-plane inviscid case having otherwise the same parameters, shown for comparison. 
work generally estimated a bottom boundary layer thickness of $30 \mathrm{~m}$, which yields a 33 day spin-down time. Wunsch (1998) argues from the shape of current-meter spectra that the barotropic mode spins down in about 100 days. However, Gille et al. (2000) estimated dissipation time scales from altimetry data of 550 to 1450 days. The author believes a better method of choosing data points which best represent the "real" ocean is to use ratios of top layer eddy to mean kinetic energy. In the $\delta=0.2,290$ day dissipation time experiment (chosen earlier as a spinup example), this ratio is 50 , which compares well with surface data in the mid-latitude regions in figure 1-1. The total eddy kinetic energy (column integrated) for this experiment is $5.7 \mathrm{~cm}^{2} \mathrm{~s}^{-2}$, which compares well with the top left panels of figures 1-3 and 1-4.

Ratios of modal kinetic energies are plotted against dissipation in figure 3-14. Note that for both values of $\delta$ the ratio reaches a limiting plateau at the viscous end. The limiting values can be predicted from a simple argument. Inversion of equations 2.14 and 2.15 yields the following relationship between the bottom layer streamfunction and the modes:

$$
\psi_{2}=\psi_{B T}-\sqrt{\delta} \psi_{B C}
$$

Suppose that when bottom Ekman friction is large, the modes correlate with each other in order to render the bottom velocities very small, so that dissipation is minimized. Figure 3-15, which plots the ratio of upper to lower layer kinetic against dissipation, confirms that the lower layer velocities are extremely small at the viscous end. Thus, to first order, we assume that $\psi_{2}$ is zero in 3.29 , which locks the modes together in the relationship:

$$
\psi_{B C}=\frac{1}{\sqrt{\delta}} \psi_{B T} \Rightarrow \frac{K E_{B C}}{K E_{B T}}=\frac{H_{2}}{H_{1}} .
$$

These are the limiting values shown in the figure. 

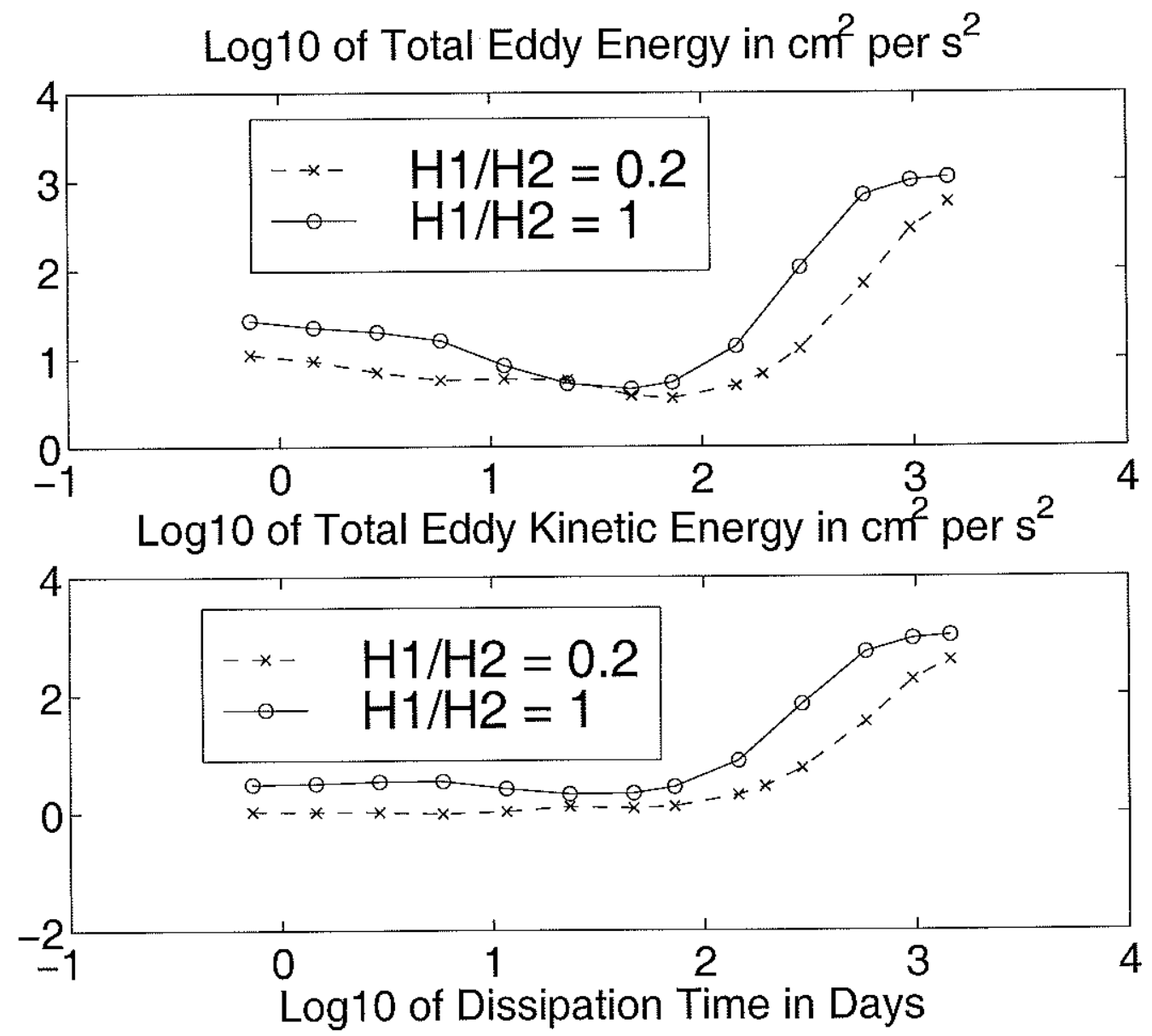

Figure 3-12: Total eddy energy (top panel) and eddy kinetic energy (bottom panel) versus dissipation time for 256 by 256 bottom-friction only f-plane zonal flow forceddissipated experiments. 


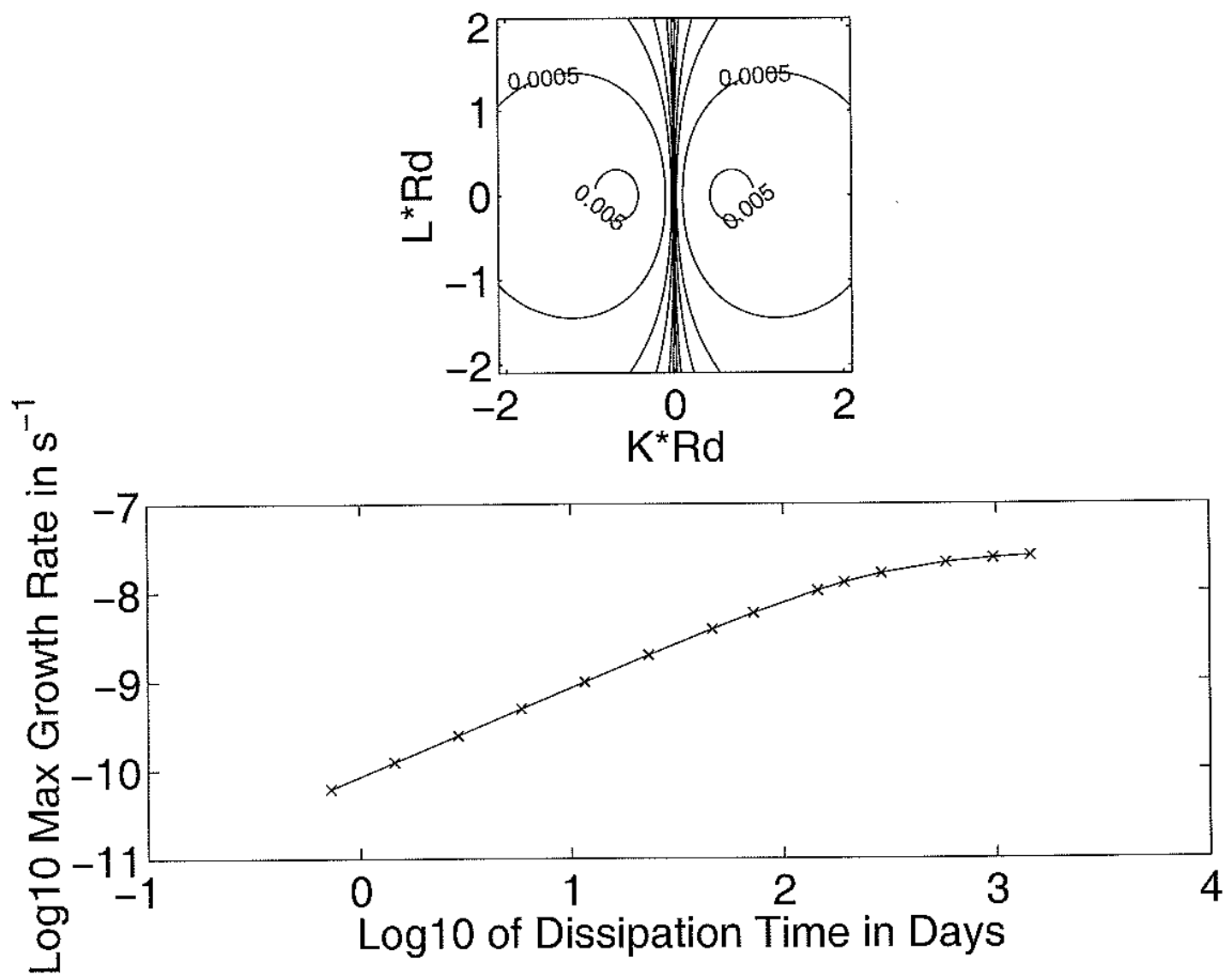

Figure 3-13: Top panel: Linear growth rates in units of $10^{-8} \mathrm{~s}^{-1}$, for the 0.7 day dissipation time bottom friction $\delta=0.2$ f-plane experiment. Bottom panel: Maximum linear growth rates as a function of Ekman friction for 256 by 256 bottom-friction only $\delta=0.2$ f-plane zonal flow forced-dissipated experiments. 


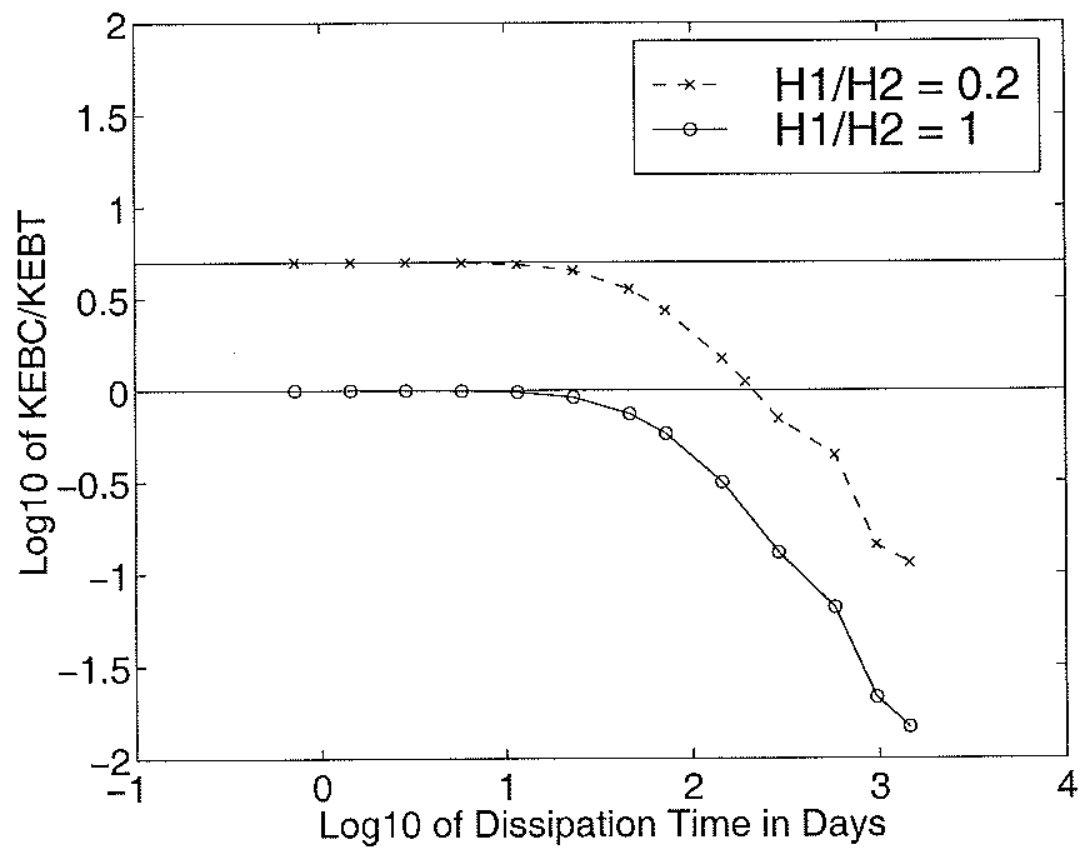

Figure 3-14: Ratios of baroclinic kinetic energy over barotropic energy versus dissipation time for 256 by 256 bottom-friction only f-plane zonal flow forced-dissipated experiments. Extra lines indicate the limiting values of 1 and $5\left(\frac{H_{2}}{H_{1}}\right.$ for the two sets of experiments).

In freely evolving experiments, eddies shift towards a barotropic state over time. Here, as friction reduces, eddies are able to evolve for some time before feeling friction, and they also tend towards a barotropic state, the more so as friction is continually reduced. This means that $\frac{K E_{B C}}{K E_{B T}}$ is bounded above by $\frac{H_{2}}{H_{1}}$. Observations (c.f. Wunsch 1997;Zang 2000;figures 1-3 and 1-4) indicate that in the mid-latitudes of both the North Pacific and North Atlantic, the first baroclinic mode contains more kinetic energy than does the barotropic mode-typically by about 50-80 percent. Our model results suggest that the first baroclinic mode will not have more kinetic energy than the barotropic mode in the case of uniform stratification, whereas it can in the case of surface-trapped stratification. Thus the surface-trapped nature of the stratification, and the bottom-trapped nature of the friction, may play a critical role in the level of eddy baroclinicity observed in the ocean.

Let us now find the nondimensional parameter which delineates the transition 


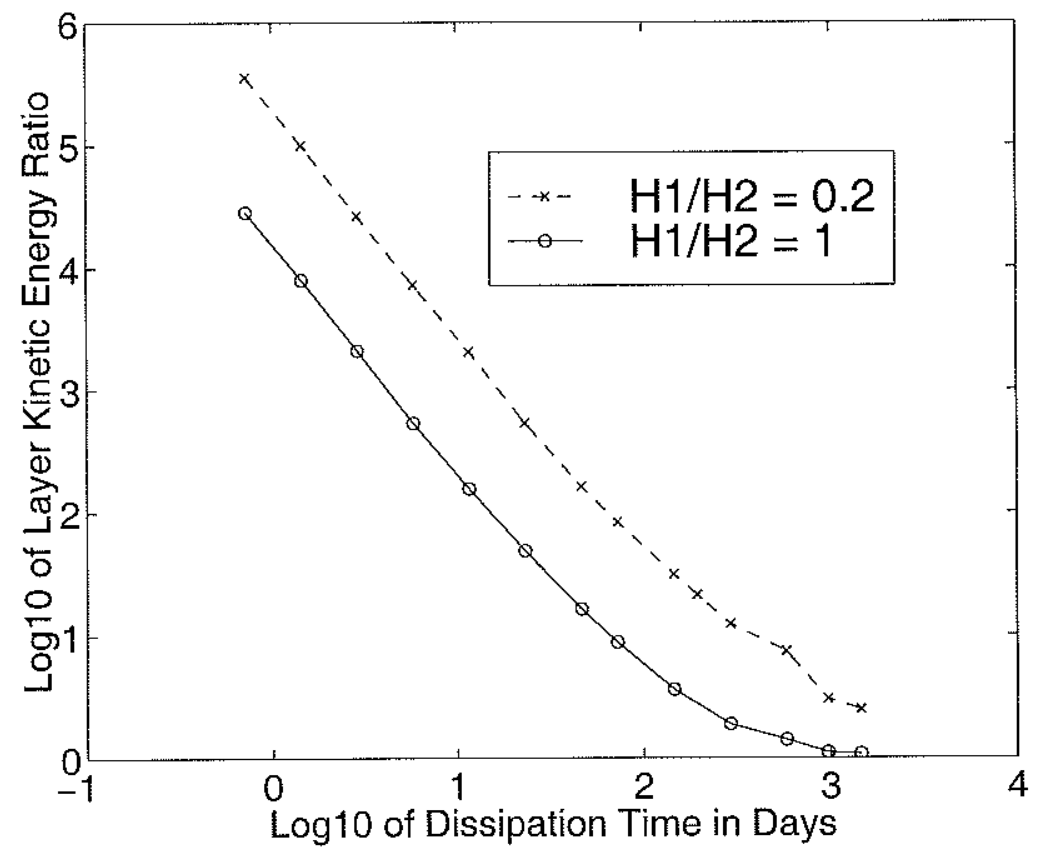

Figure 3-15: Ratios of upper layer to lower layer kinetic energies versus dissipation time for 256 by 256 bottom-friction only f-plane zonal flow forced-dissipated experiments.

between friction-dominated and nonlinear cascade-dominated regimes. We scale the Ekman friction term and the nonlinear Jacobian term in the bottom layer PV equation 2.2:

$$
\begin{gathered}
\text { friction }=-R_{2} \nabla^{2} \psi_{2}, \\
\text { nonlinear }=J\left(\psi_{2}, \nabla^{2} \psi_{2}+\frac{\delta\left(\psi_{1}-\psi_{2}\right)}{(1+\delta) R_{d}^{2}}\right) .
\end{gathered}
$$

We have seen that upper layer velocities are always greater than lower layer velocities. We will see later that eddy length scales are always larger than $R_{d}$. For simplicity, then, let us write:

$$
\text { nonlinear } \sim J\left(\psi_{2}, \frac{\delta \psi_{1}}{(1+\delta) R_{d}^{2}}\right) \Rightarrow \frac{\text { nonlinear }}{\text { friction }} \sim \frac{\frac{\psi_{2}}{L_{2}} \frac{\delta \psi_{1}}{(1+\delta) R_{d}^{2} L_{1}}}{\frac{R_{2} \psi_{2}}{L_{2}^{2}}}
$$


where the Jacobian has picked out separate layer eddy length scales $L_{1}$ and $L_{2}$. If we scale the upper layer eddy velocities by $U_{1}$ and use $\frac{\psi_{1}}{L_{1}} \sim U_{1}, 3.32$ simplifies to:

$$
\frac{\text { nonlinear }}{\text { friction }} \sim \frac{\delta U_{1} L_{2}}{(1+\delta) R_{d}^{2} R_{2}} .
$$

As we will see, in the transition regime, eddy scales in both layers are close to $R_{d}$. So if $R_{d}$ and $L_{2}$ are both set to an eddy length scale $L$, and the $\delta$ factors are ignored, 3.33 further simplifies to:

$$
\frac{\text { nonlinear }}{\text { friction }} \sim \frac{U_{1}}{L R_{2}} \sim \frac{T_{\text {friction }}}{T_{\text {eddy }}} .
$$

Nonlinear terms dominate when eddy turnover time is small compared to frictional time, and friction dominates when the eddy turnover time is large compared with the dissipation time. This last exercise helps us understand the meaning of the scaling in a simple limit. However, we will use 3.33 as our nondimensional parameter because it does not assume that the length scales are order $R_{d}$, so it will hold more generally over the range of friction values used. The top half of figure 3-16 plots the baroclinicity ratios against this parameter. The transition occurs when the nonlinear and friction terms are of the same order. When the nonlinear term is much larger than friction, eddies cascade to a barotropic state as friction reduces. When friction dominates, eddy baroclinicities take on the predicted limiting value $\frac{H_{2}}{H_{1}}$. This parameter is a function of $L_{2}$ and $U_{1}$, which are not known a priori, since they are part of the solution of the nonlinear equilibration problem. Comparison of the top halves of figures 3-11 and 3-13 indicates that linear stability properties undergo a qualitative transition as friction changes. Namely, a destabilization of short waves occurs as friction increases, as evidenced by the fact that in the latter plot, growth rates at high wavenumbers are larger fractions of the maximum growth rates than is the case in the former plot. The linear analysis, of course, cannot know about $L_{2}$ and $U_{1}$; it must depend on $\frac{\overline{u_{1}}-\overline{u_{2}}}{R_{d} R_{2}}$ (and on $\delta$, but since that is order one 
we omit it). The bottom half of figure 3-16 plots ratios of the nonlinear solutions against $\frac{\overline{u_{1}}-\overline{u_{2}}}{R_{d} R_{2}}$. The regime transition occurs when this quantity is order one; thus the value of friction at the transition can be roughly predicted without knowing any of the characteristics of the nonlinear problem.

Ratios of modal kinetic energies are plotted against total eddy energy in figure 317. The regime of non-monotonic energy versus friction shows up as a "hook" on the plots. Outside this regime, barotropization increases with increasing energy. Plotting baroclinicities as a function of total eddy energy rather than dissipation time brings the curves for the two different $\delta$ values closer together; the curves collapse together even more strikingly in the next section. An offset exists between the baroclinicity ratios; the $\delta=0.2$ experiments are more baroclinic for a fixed value of total eddy energy, typically by a factor of about two. Note that $\frac{K E_{B C}}{K E_{B T}}=0.7$ in the $\delta=0.2290$ day dissipation time experiment, which we have chosen as having a realistic ratio of eddy to mean kinetic energy. The baroclinicity ratio is too low; the 145 day experiment has a baroclinic to barotropic ratio of 1.5 , closer to mid-ocean values. For this experiment the ratio of eddy to mean kinetic energy is 22 , which is somewhat small compared to mid-ocean values (figure 1-1). The model is not quite baroclinic enough for realistic energies, when compared to the actual ocean.

Plots of total baroclinic energy over barotropic energy (not shown) paint a similar picture, as do the enstrophies. Total potential enstrophy (normalized by $f^{2}$, where $f$ takes the nominal value $10^{-4} \mathrm{~s}^{-1}$ ) is plotted versus dissipation in figure $3-18$. Nonmonotonic behavior with friction is evident. The ratio of $\left(\nabla^{2} \psi_{B C}\right)^{2}$ over $\left(\nabla^{2} \psi_{B T}\right)^{2}$ is plotted against the ratio of nonlinear to Ekman terms in the top half of figure 3-19. When Ekman friction dominates, the enstrophy baroclinicity ratio takes on the same limiting values as does the kinetic energy ratio. Barotropization of enstrophies is observed as nonlinear terms begin to dominate, but the amount of barotropization is considerably less than when energy ratios are the norm. When the enstrophy ratio is plotted against total potential enstrophy, in the bottom half of figure 3- 

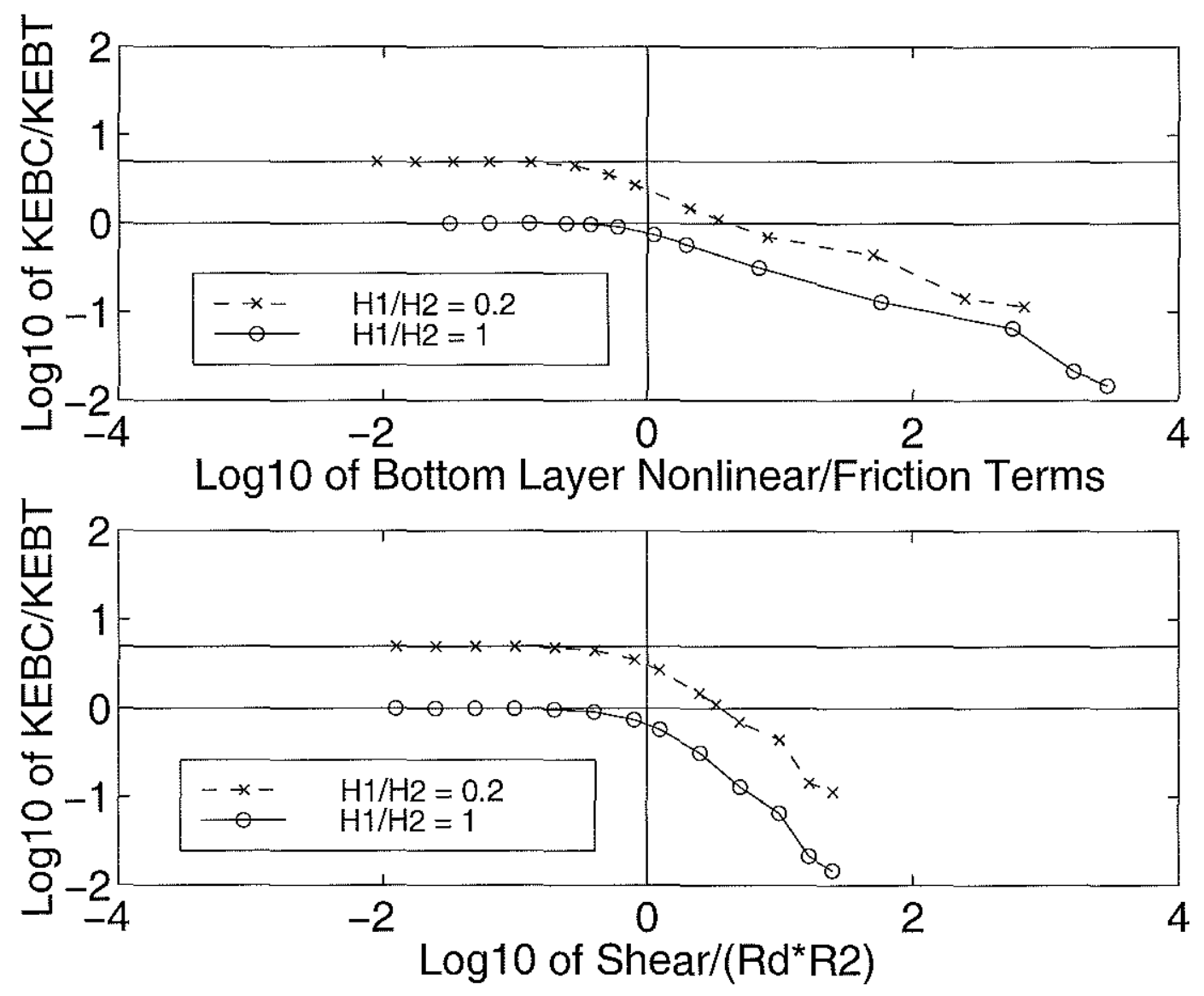

Figure 3-16: Ratios of baroclinic to baroclinic kinetic energy versus $\frac{\delta U_{1} L_{2}}{(1+\delta) R_{2} R_{d}^{2}}$ (top panel) and versus $\frac{\overline{u_{1}}-\overline{u_{2}}}{R_{d} R_{2}}$ (bottom panel) for 256 by 256 bottom-friction only f-plane zonal flow forced-dissipated experiments. Extra horizontal lines indicate the limiting values of $\frac{H_{2}}{H_{1}}$. The extra vertical line indicates that the transition between frictiondominated and cascade dominated regimes occurs when either parameter is order one. 


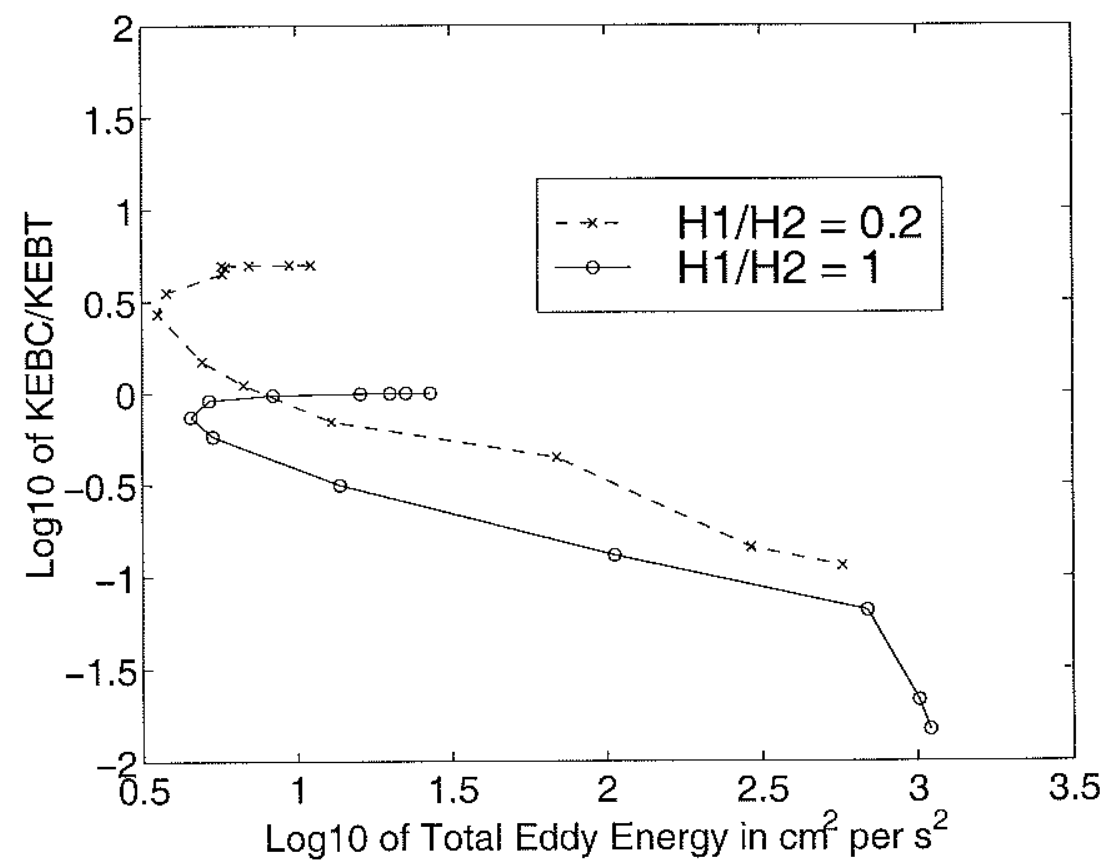

Figure 3-17: Ratios of modal kinetic energies versus total eddy energy for 256 by 256 bottom-friction only f-plane zonal flow forced-dissipated experiments.

19 , an offset exists between the $\delta=0.2$ and $\delta=1$ experiments, indicating that eddy baroclinicity as measured by modal enstrophy ratios is also enhanced with surface-trapped stratification. The ratio of $\left(\nabla^{2} \psi_{B C}\right)^{2}+\frac{1}{R_{d}^{2}}\left(\nabla \psi_{B C}\right)^{2}$ (the part of the baroclinic enstrophy which feels Ekman friction and is thus weakly damped under surface-trapped stratification-c.f. equation 2.30) over $\left(\nabla^{2} \psi_{B T}\right)^{2}$, and the ratio of total baroclinic potential enstrophy to barotropic enstrophy, tell the same story and are not shown for the sake of brevity.

Figure 3-20 plots Ekman dissipation of energy and enstrophy over PV flux production-see equations 2.29 and 2.30. Ekman friction balances energy production to within about ten percent, meaning that the wavenumber filter makes little difference to the energy balance. However, the same is not true of the enstrophy balance. At the viscous end, the fraction of enstrophy dissipated by bottom Ekman friction takes on a limiting value of $\frac{\delta}{1+\delta}$. As friction reduces, this fraction decreases. This can be understood with the following simple argument, based on the layer po- 


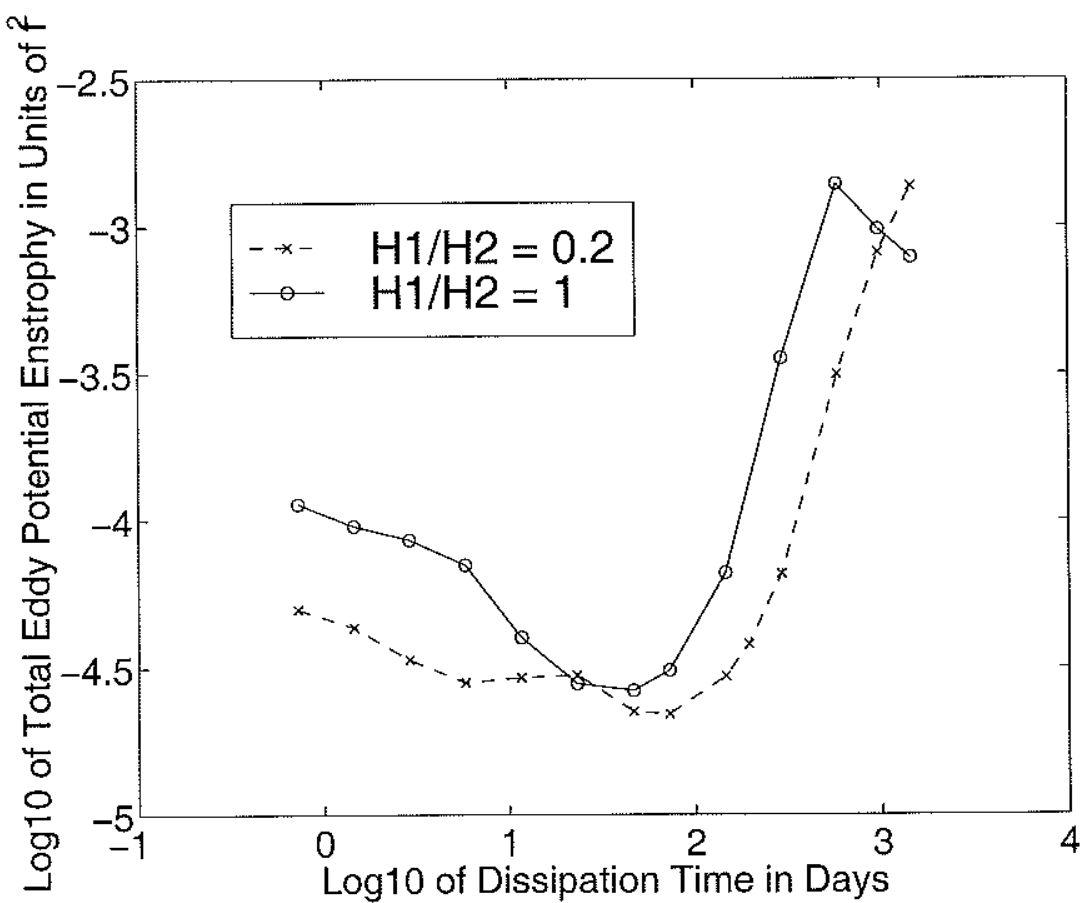

Figure 3-18: Total potential enstrophy versus dissipation time for 256 by 256 bottomfriction only f-plane zonal flow forced-dissipated experiments.

tential enstrophy equations 2.26 and 2.27. These equations, under the conditions of the present experiments, and under the assumption of a statistically steady state, are:

$$
\begin{gathered}
\frac{\delta\left(\overline{u_{1}}-\overline{u_{2}}\right)}{(1+\delta) R_{d}^{2}} \iint q_{1} \frac{\partial \psi_{1}}{\partial x} d x d y=f_{1}, \\
-\frac{\delta\left(\overline{u_{1}}-\overline{u_{2}}\right)}{(1+\delta) R_{d}^{2}} \iint q_{2} \frac{\partial \psi_{2}}{\partial x} d x d y=f_{2}+E_{q},
\end{gathered}
$$

where $f_{1}$ and $f_{2}$ denote depth-integrated enstrophy dissipations by the wavenumber filter in the top and bottom layers, respectively, and $E_{q}$ denotes depth-integrated enstrophy dissipation by bottom Ekman friction. Combining these with equation 2.23 yields $E_{q}+f_{2}=\delta f_{1}$, which implies that: 


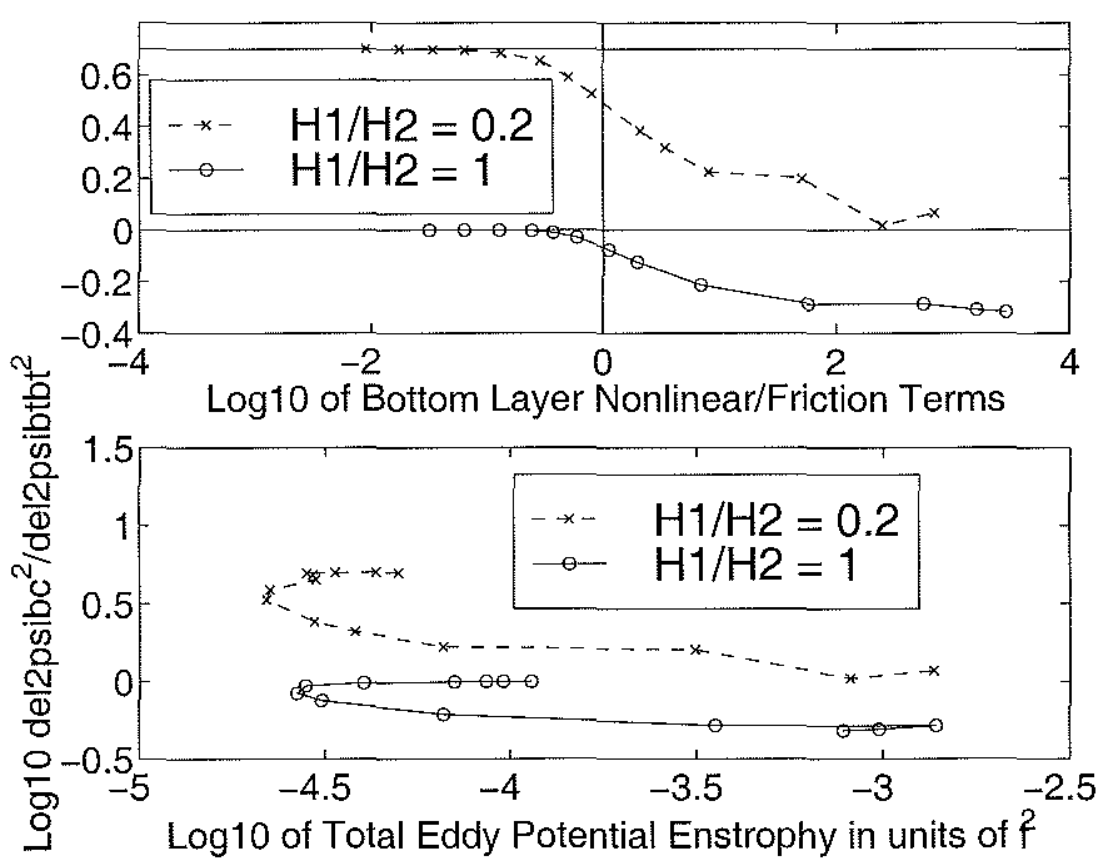

Figure 3-19: $\left(\nabla^{2} \psi_{B C}\right)^{2}$ over $\left(\nabla^{2} \psi_{B T}\right)^{2}$ versus $\frac{\delta U_{1} L_{2}}{(1+\delta) R_{2} R_{d}^{2}}$ (top half) and versus total potential enstrophy (bottom half) for 256 by 256 bottom-friction only f-plane zonal flow forced-dissipated experiments. Extra lines indicate limiting values of 1 and 5 .

$$
\frac{E_{q}}{E_{q}+f_{1}+f_{2}}=\frac{\delta}{1+\delta}-\frac{f_{2}}{(1+\delta) f_{1}}
$$

In experiments with large bottom friction, if one assumes that $f_{2}$ is nearly zero, i.e. that Ekman friction dominates the bottom layer enstrophy dissipation, then the left hand side goes to $\frac{\delta}{1+\delta}$, exactly the ratio that is seen in the figures. On the other hand, as Ekman friction decreases, the nonlinear term dominates and one expects a strong cascade. Then $f_{2}$ becomes important and the fraction accounted for by Ekman friction decreases, also in agreement with the figures. 

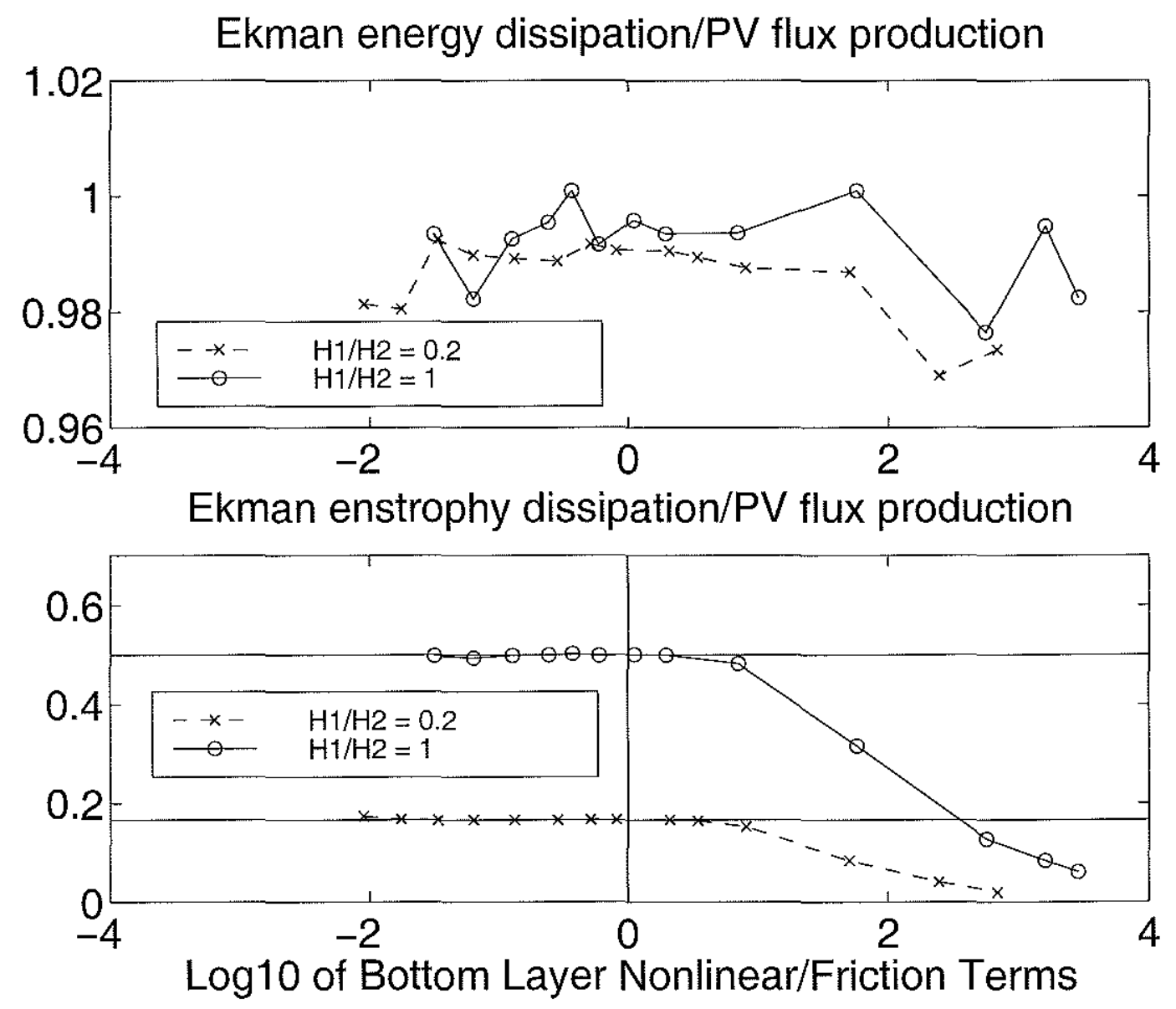

Figure 3-20: Ekman dissipation of energy (top panel) and potential enstrophy (bottom panel) over PV flux production for 256 by 256 bottom-friction only f-plane zonal flow forced-dissipated experiments. In the lower panel, lines are drawn at the values $1 / 2$ and $1 / 6$, i.e. $\frac{\delta}{1+\delta}$ for the two values of $\delta$. 


\subsection{Baroclinicity in forced-dissipated experiments with symmetric friction}

The results of the last section indicate that surface-trapped stratification enhances eddy baroclinicity in bottom-friction only forced-dissipated experiments. We began with two arguments as to why this should be so, one having to do with nonlinear interactions between modes and one having to do with damping of the modes. Both mechanisms are present in the bottom friction only experiments. Friction was proven important by the fact that the upper bound of the ratio $\frac{K E_{B C}}{K E_{B T}}$ can be calculated from a frictional argument. The nonlinear interaction mechanism was shown to be important in freely evolving experiments. Symmetric friction experiments can shed further light on the importance of the two mechanisms in a forced-dissipated system. In these experiments surface-trapped stratification enhances eddy baroclinicity only through the nonlinear projection mechanism.

Brute-force application of the linear stability code indicates that there is a cutoff value of friction in the symmetric case. When friction increases beyond this cutoff value, the flow is linearly stable. This is consistent with earlier studies (c.f. Barcilon 1964; Williams and Robinson 1974) and with model behavior; eddy energy was found to decay when friction is increased beyond the cutoff value.

As in the bottom friction only experiments, the value of Ekman friction was varied over a wide range in the symmetric friction experiments. Total eddy energy and eddy kinetic energy are plotted as a function of dissipation time in figure 321. Non-monotonic behavior is once again evident, and more so in the total energy plot than the kinetic energy plot. Here the non-monotonic behavior occurs near a stability boundary. Since there is no stability boundary in the bottom friction only case, it is not clear to this author that the reasons for non-monotonic behavior are the same under the different types of friction. The non-monotonic behavior has not been explained in either case. 


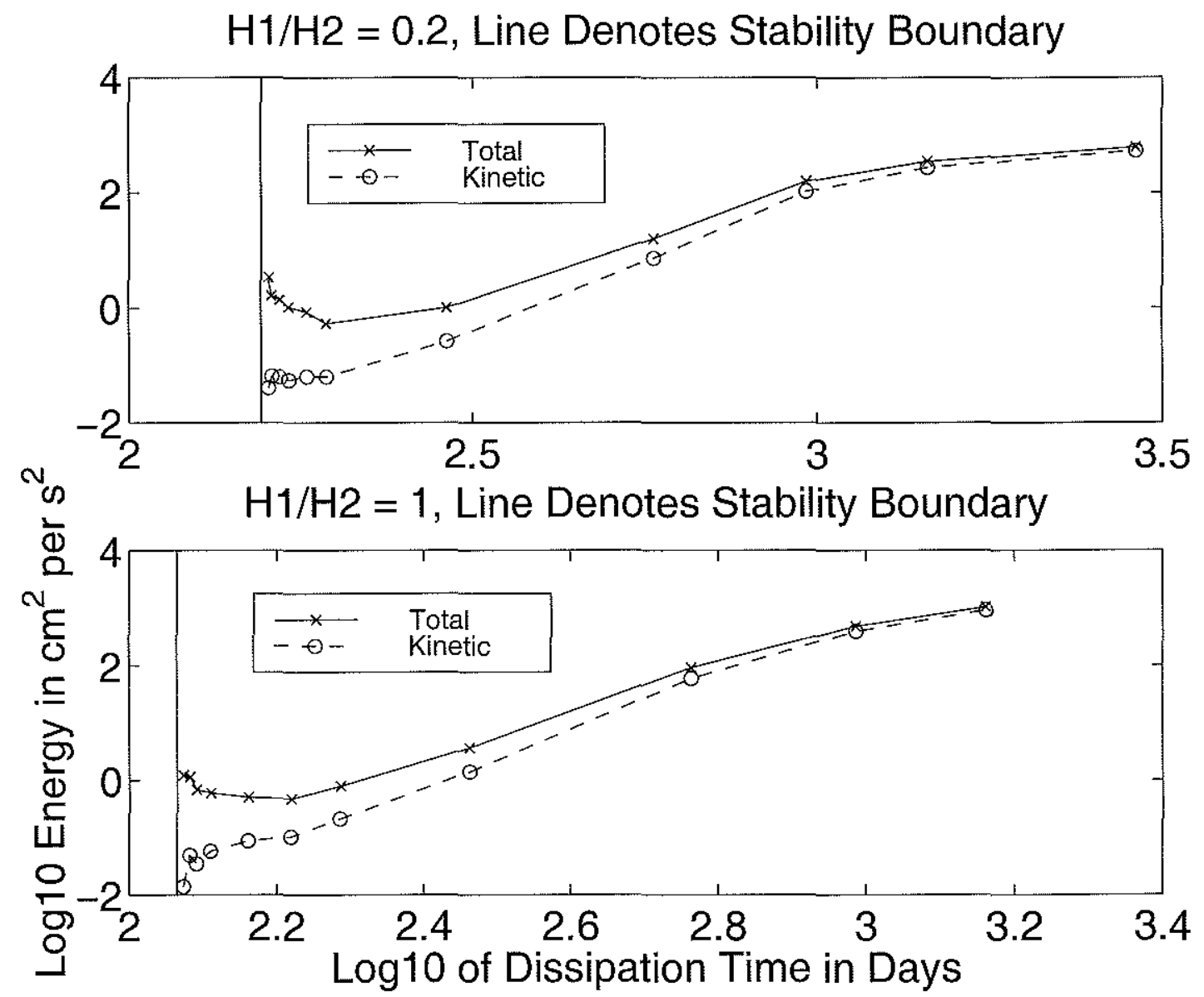

Figure 3-21: Total eddy energy and eddy kinetic energy versus dissipation time for 256 by $256 \delta=0.2$ (top panel) and $\delta=1$ (bottom panel) symmetric friction f-plane zonal flow forced-dissipated experiments. 
Ratios of modal kinetic energies are plotted versus total eddy energy in figure 322. Once again, eddies barotropize with increasing energy. Offsets between the $\delta=0.2$ and $\delta=1$ experiments over most of the energy range indicate enhancement of eddy baroclinicity by surface-trapped stratification. However, the offset is much less than in the bottom-friction only experiments (figure 3-17). This contrast further suggests that surface-trapped stratification enhances eddy baroclinicity in the bottom-friction only experiments mainly because of the weak damping of the baroclinic mode, rather than through differences in nonlinear interactions between modes. Ratios of total baroclinic energy to barotropic energy-not shown-give similar results. Figure 3-23 plots baroclinicity of eddy energies versus dissipation time. For both values of $\delta$, baroclinicity increases as friction increases towards the stability boundary. In fact $\frac{K E_{B C}}{K E_{B T}}$ takes on larger values than any achieved in the bottom friction experiments.

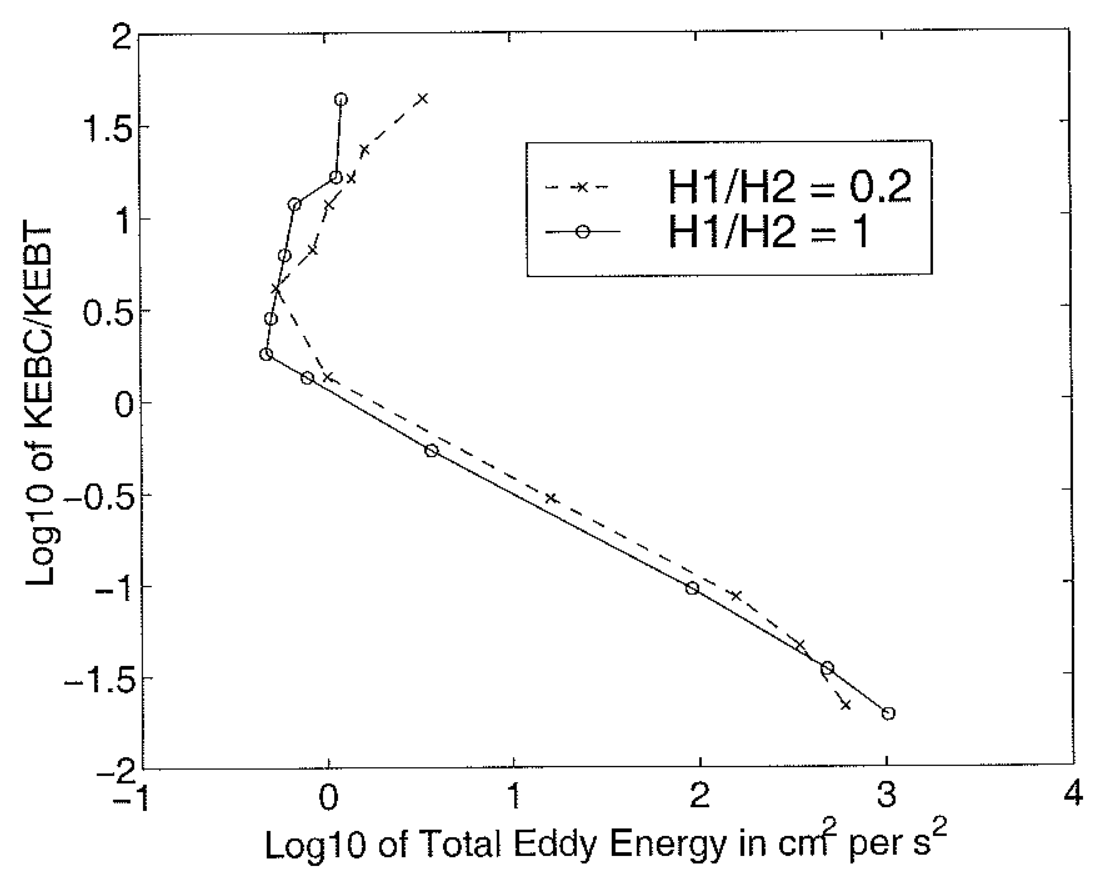

Figure 3-22: Ratios of modal kinetic energies versus total eddy energy for 256 by 256 symmetric friction f-plane zonal flow forced-dissipated experiments.

The same modal enstrophy partition plotted in the bottom friction case is plotted 
Delta 0.2

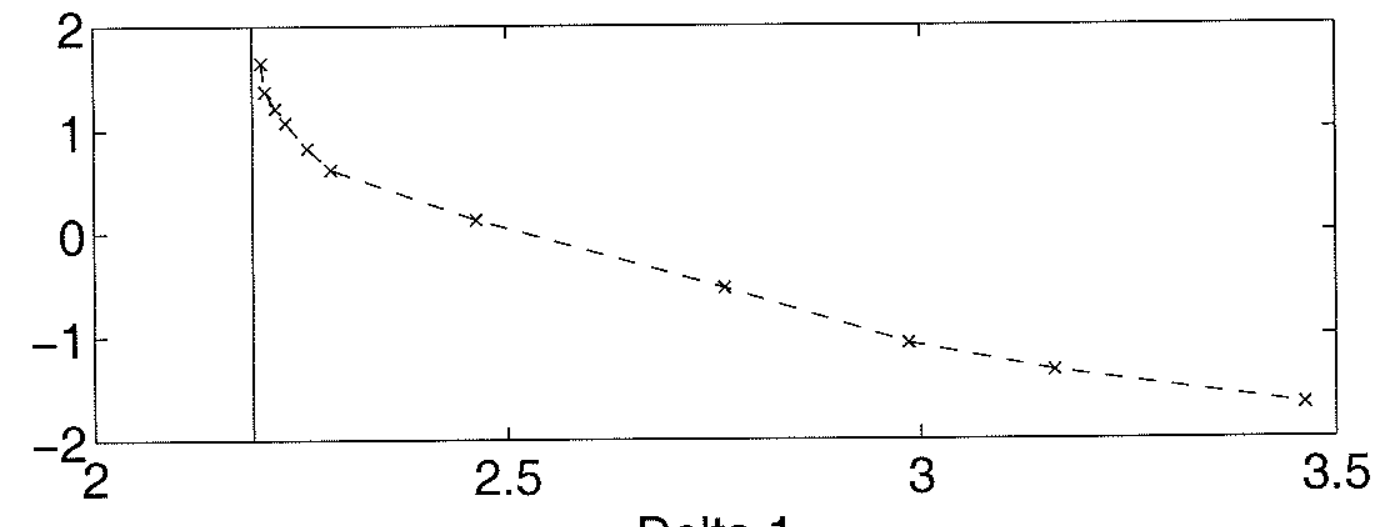

Delta 1

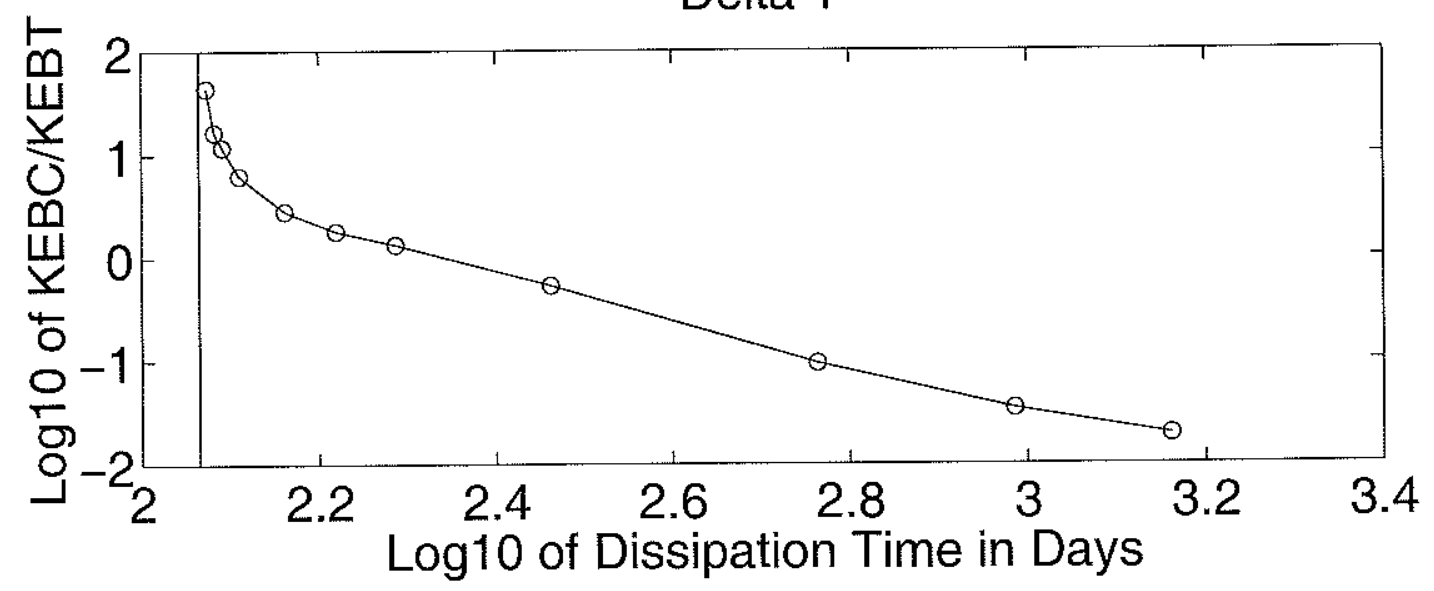

Figure 3-23: Ratios of modal kinetic energies versus dissipation time for 256 by 256 $\delta=0.2$ (top panel) and $\delta=1$ (bottom panel) symmetric friction f-plane zonal flow forced-dissipated experiments. 
versus total enstrophy for the symmetric friction case in figure 3-24. Offsets between $\delta=0.2$ and $\delta=1$ experiments are more noticable in the enstrophies than in the energies, and more comparable to offsets in the bottom friction only experiments. The nonlinear projection mechanism apparently becomes more important when the baroclinicity measure is enstrophy rather than energy, consistent with the fact that Ekman friction does not dominate dissipation of enstrophy as it does dissipation of energy.

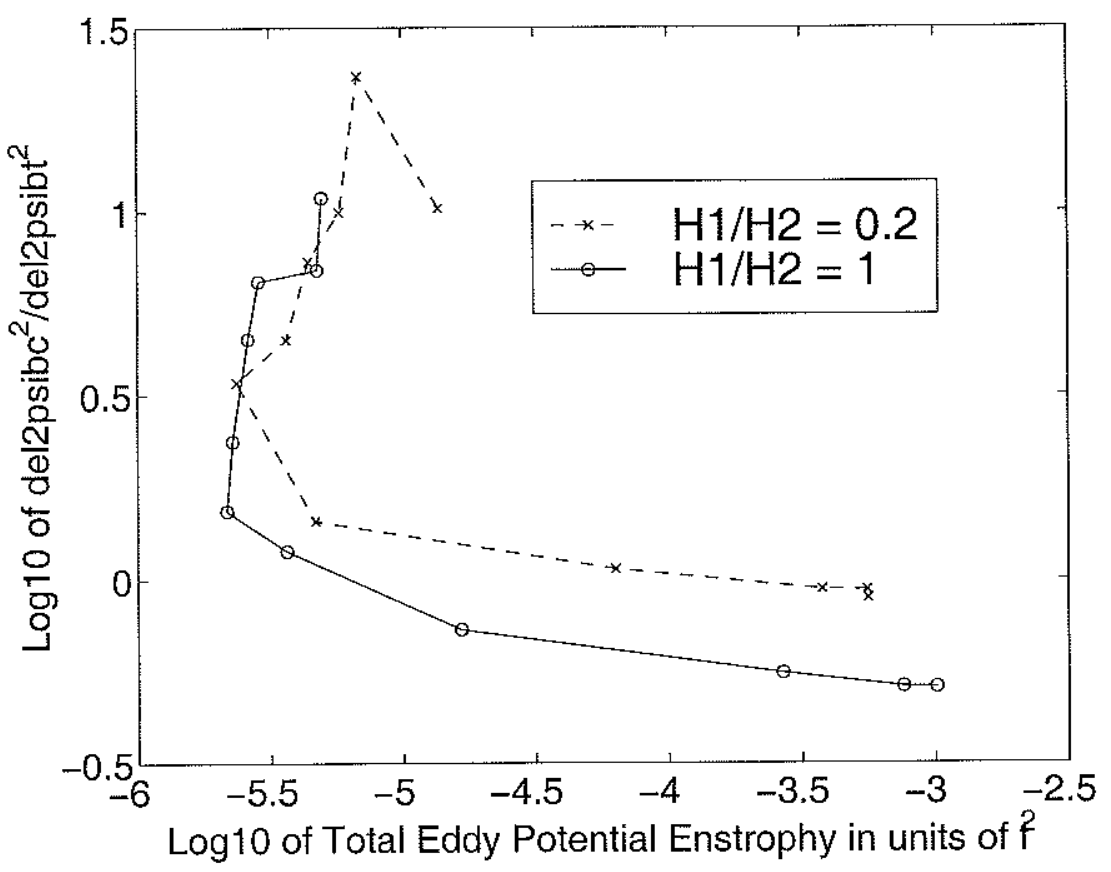

Figure 3-24: $\left(\nabla^{2} \psi_{B C}\right)^{2}$ over $\left(\nabla^{2} \psi_{B T}\right)^{2}$ versus total potential enstrophy for 256 by 256 symmetric friction f-plane zonal flow forced-dissipated experiments. 


\subsection{A word about friction in the ocean}

This chapter of the thesis has emphasized the importance of the bottom-trapped nature of friction in enhancing levels of eddy baroclinicity in the models, and, perhaps, the actual ocean. Under the action of linear bottom Ekman friction, which we use in this thesis, dissipation of kinetic energy is proportional to the energy itself. This makes for simple interpretation of model behavior, but one might wonder how realistic it is. Derivation of the lower layer QG PV equation can be done as follows. Starting from the vorticity balance (c.f. Pedlosky 1987):

$$
\left[\frac{\partial}{\partial t}+J\left(\psi_{2}, \bullet\right)\right] \nabla^{2} \psi_{2}=f_{0} \frac{\partial w}{\partial z},
$$

one then integrates over the bottom layer of depth $H_{2}$ to obtain:

$$
\left[\frac{\partial}{\partial t}+J\left(\psi_{2}, \bullet\right)\right] \nabla^{2} \psi_{2}=f_{0} \frac{w_{\text {interface }}-w_{\text {topbl }}}{H_{2}},
$$

where $w_{\text {interface }}$ is the vertical velocity at the layer interface and leads to the vortex stretching term, while $w_{\text {topbl }}$ is the vertical velocity at the top of the bottom boundary layer. In "classical" Ekman layers, $w_{\text {topbl }}$ is proportional to the vorticity of the flow above the boundary layer, leading to a simple linear friction. Work on bottom boundary layers suggests that bottom stress $\tau$ is proportional to the square of the turbulent velocity scale, rather than the velocity itself, as is assumed in classical Ekman theory. Assuming the former rather than the latter leads to a complicated nonlinear relationship between Ekman pumping velocity and background flow (c.f. page 329 of Gill 1982). Over rough topography, energy dissipation may be dominated by internal wave generation and subsequent breaking (c.f. Polzin et al. 1997; Gille et al. 2000). This, too, may lead to more complicated relationships. With these mechanisms, it would still be true that dissipation occurs at the bottom, and probably still true that the baroclinic mode in a surface-trapped stratification would 
be weakly damped. It would be difficult to show this analytically, but perhaps not so difficult to show in numerical models. A study similar to the present one but with more realistic dissipation schemes is a possible avenue of future investigation. The biggest problem when taking that route would be to decide exactly what is a realistic form of the friction. This has always been a difficult problem for oceanographers, and is a matter of ongoing research.

\subsection{Appearance and length scales of model eddy fields}

In this section we examine the appearance of model eddy fields discussed in the preceding sections. We also take a quantitative look at eddy length scales. We expect that eddy fields which are strongly baroclinic will also have compact horizontal scales, since the baroclinic mode is associated with $R_{d}$. This association can be seen in the experiment contoured in figure $3-25$, in which the baroclinic streamfunction is at substantially smaller scales than the barotropic streamfunction.

Snapshots of upper layer fluctuation streamfunctions for the $\delta=0.2$ bottomfriction only experiments are shown in figures 3-26 through 3-29. The length scales of the most viscous experiment are relatively large, and the field is generally smooth in appearance. Surprisingly, small-scale fronts are noticable. This may be related to the destabilization of small scales by large bottom friction, as discussed earlier. Destabilization is demonstrated in figure 3-30, which plots the ratio of the growth rate for a wave with meridional wavenumber $l=0$ and zonal wavenumber $k=\frac{\pi}{R_{d}}$ (i.e. a wavelength of $2 R_{d}$ ), over the maximum growth rate, as a function of $\frac{\overline{u_{1}}-\overline{u_{2}}}{R_{2} R_{d}}$. A transition occurs when this parameter is order one. For small frictions, the growth

at our chosen small scale of $\frac{R_{d}}{\pi}$ is much less than the maximum growth achieved in the domain. For large frictions, the growth at small scales is a much greater fraction of the maximum growth rate. Thus one might expect small scales to be energized in 


\section{Upper Layer}

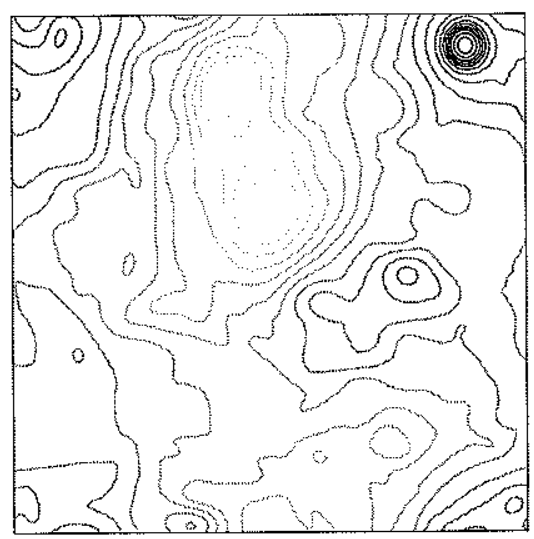

\section{Barotropic}

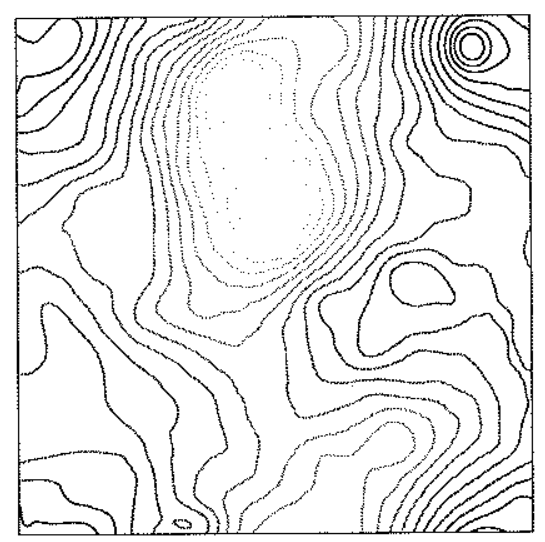

Lower Layer

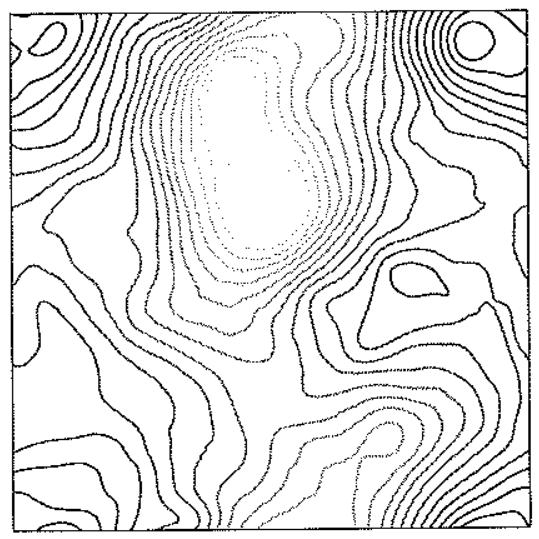

Baroclinic

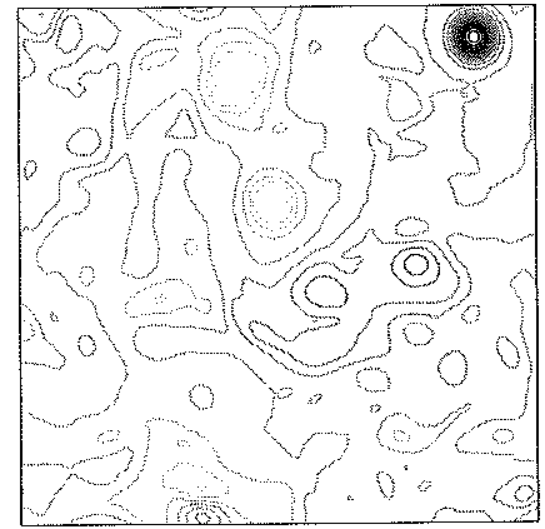

Figure 3-25: Instantaneous snapshots of fluctuation layer and modal streamfunctions for $\delta=0.2$ f-plane forced-dissipated experiment with 967.2 day bottom friction dissipation time. 
the most viscous experiments. As friction reduces, the fronts contort and eventually break up, transiting smoothly into a regime of densely packed vortices (starting at dissipation times of 23.2 and 46.4 days) more typical of the f-plane geostrophic turbulence literature (c.f. Larichev and Held 1995). The current study is apparently the first to push into this highly viscous regime. The smooth transition of the streamfunction appearance, as well as of integral quantities such as $\frac{K E_{B C}}{K E_{B T}}$ (which are easily predictable in the viscous regime), lead this author to guess that more study of the viscous regime might shed light on the behavior of the whole range of forceddissipated f-plane geostrophic turbulence. The vortex regime in the mid-range of friction values eventually cascades to the domain scale as friction is further reduced. This is consistent with the long-time behavior of freely evolving experiments. Note that none of these experiments could be described as "wavelike" in appearance. The $\delta=1$ bottom friction only experiments are not shown here for the sake of brevity. Their behavior is similar. 


\subsection{5 day dissipation}

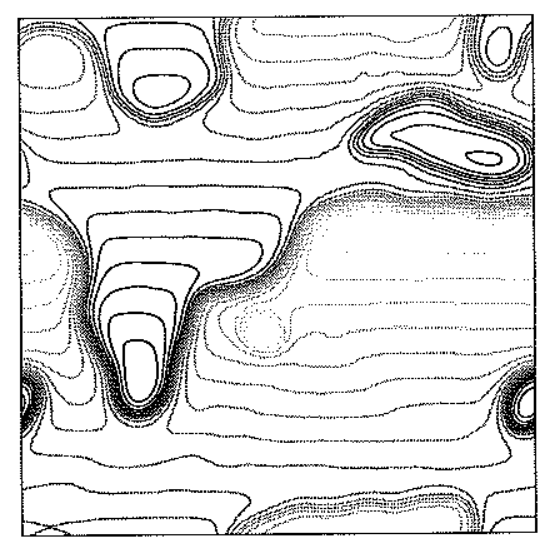

2.90 day dissipation

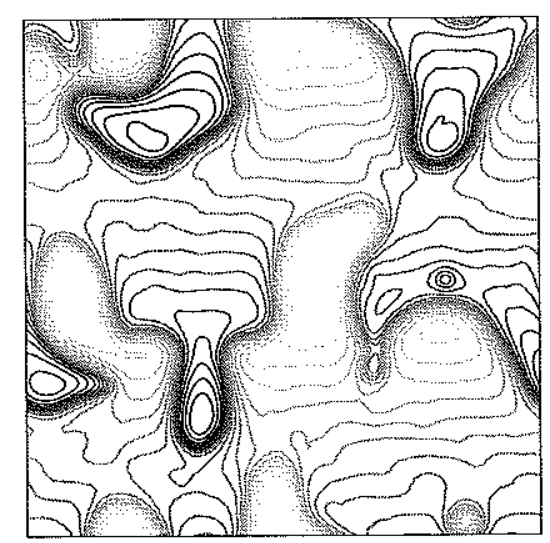

\subsection{5 day dissipation}

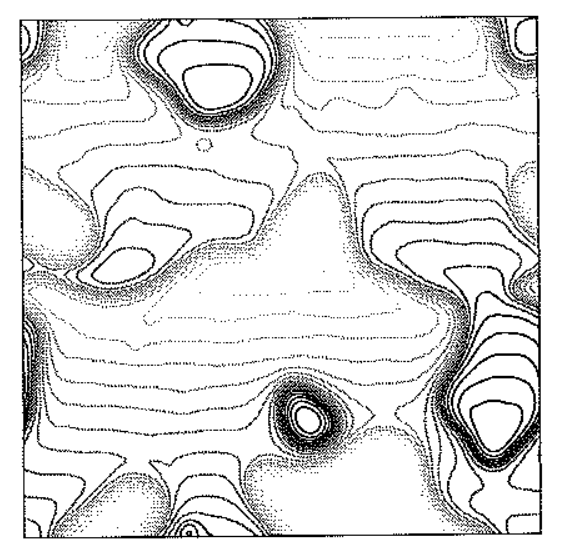

5.80 day dissipation

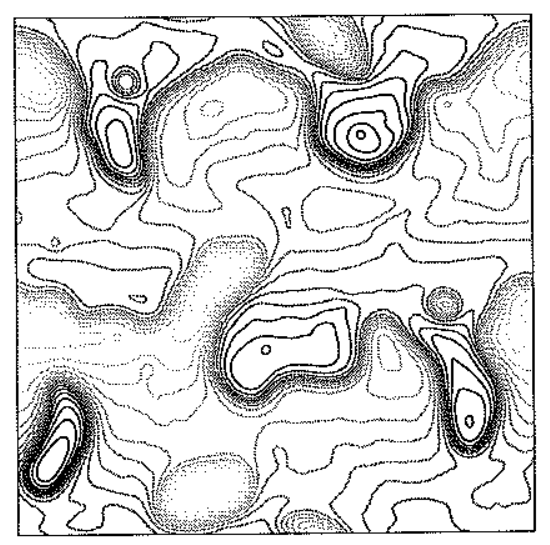

Figure 3-26: Contours of snapshots of top layer fluctuation streamfunction for the bottom friction only $\delta=0.2$ experiments. 


\section{6 day dissipation}

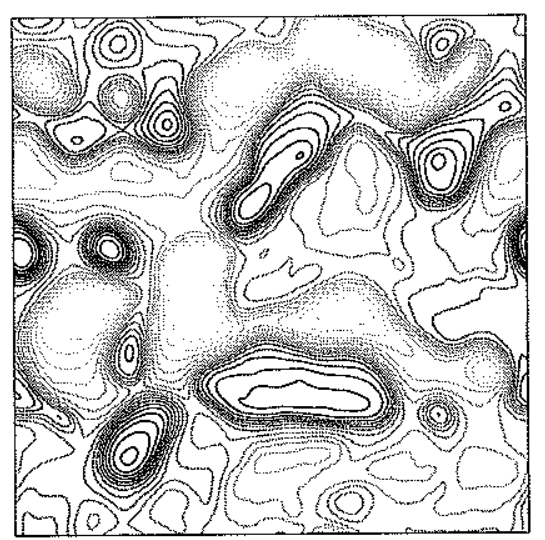

\section{4 day dissipation}

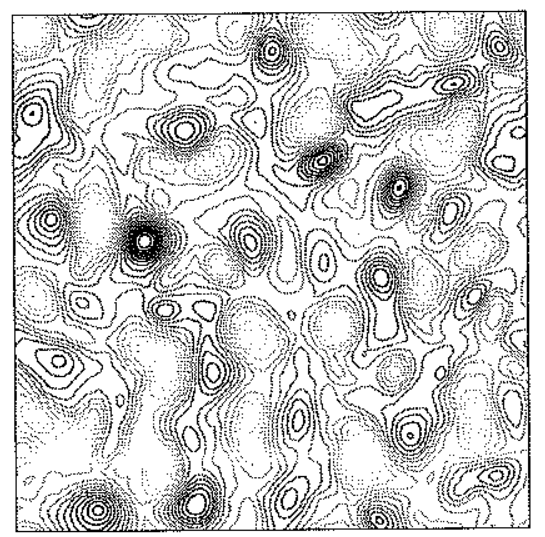

23.2 day dissipation

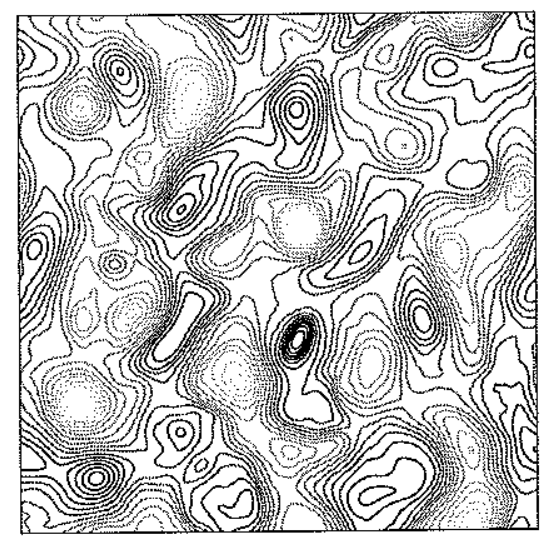

72.5 day dissipation

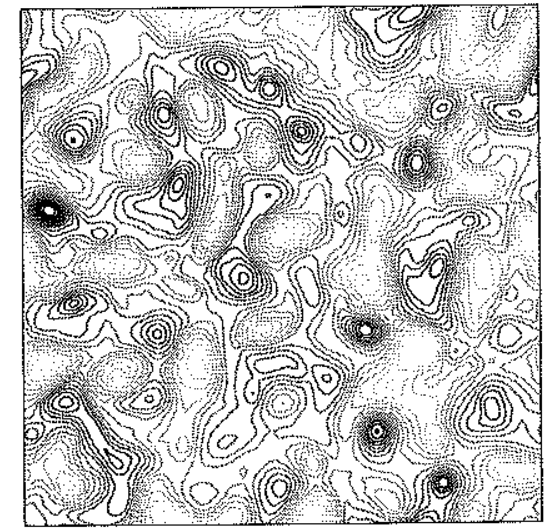

Figure 3-27: Contours of snapshots of top layer fluctuation streamfunction for the bottom friction only $\delta=0.2$ experiments, continued. 


\section{1 day dissipation}

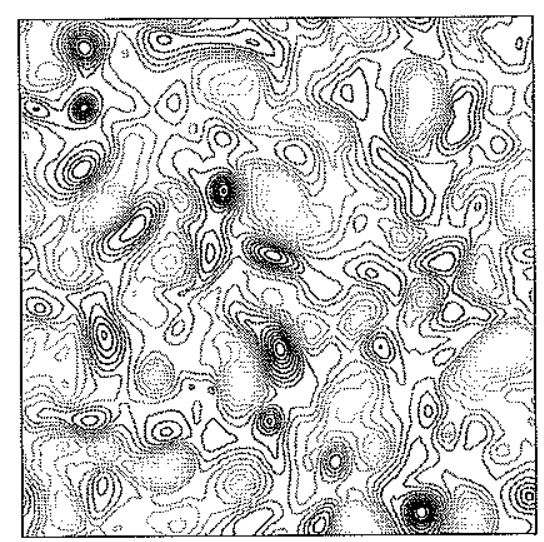

290.2 day dissipation

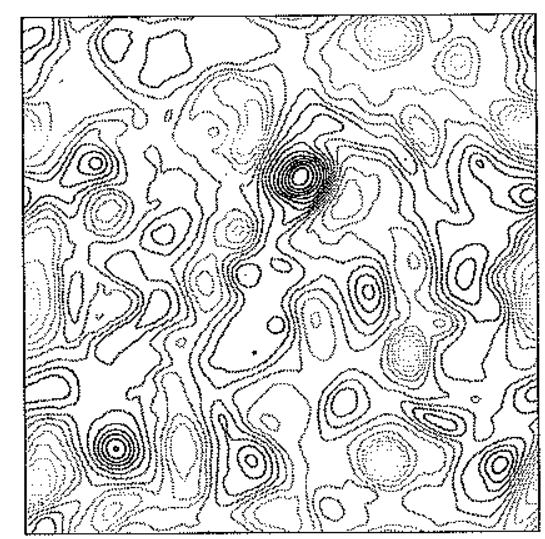

193.4 day dissipation

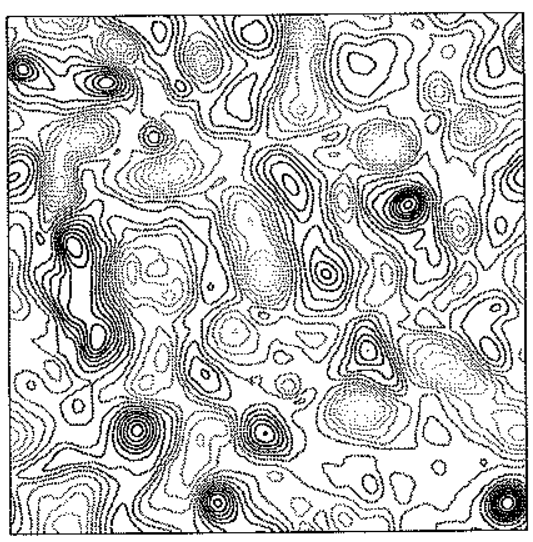

580.3 day dissipation

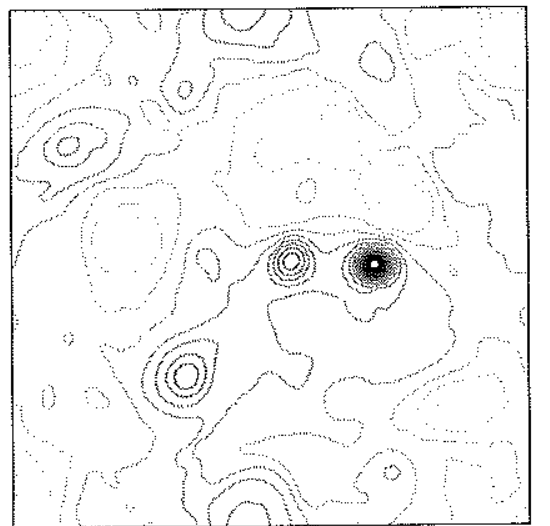

Figure 3-28: Contours of snapshots of top layer fluctuation streamfunction for the bottom friction only $\delta=0.2$ experiments, continued. 
967.2 day dissipation

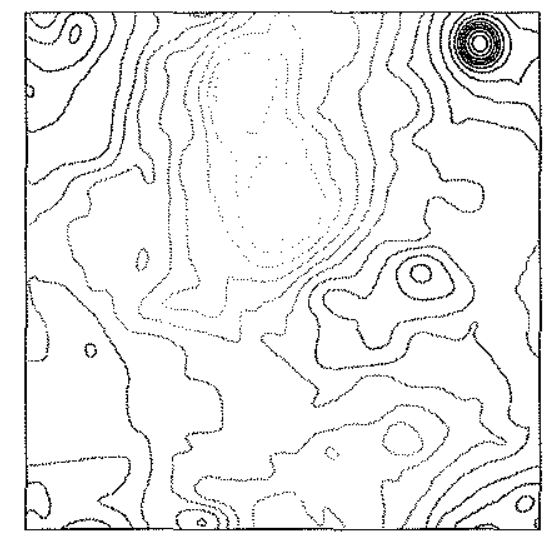

1450.8 day dissipation

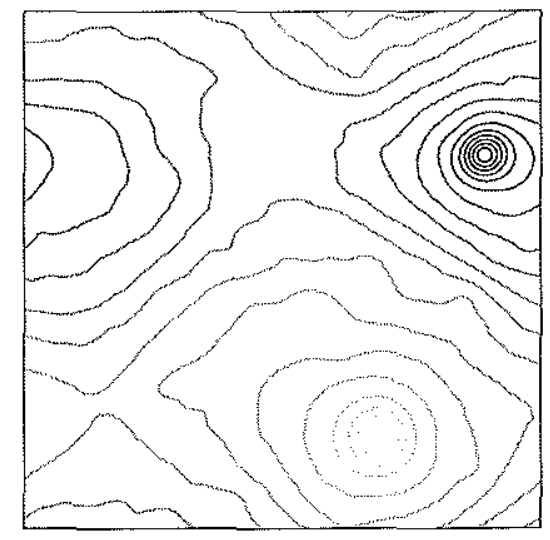

Figure 3-29: Contours of snapshots of top layer fluctuation streamfunction for the bottom friction only $\delta=0.2$ experiments, continued.

The $\delta=0.2$ symmetric friction experiments have snapshots of their upper layer fluctuation streamfunctions plotted in figures 3-31 through 3-33. Experiments having frictional values close to the stability cutoff exist in a regime of long weakly nonlinear waves. Figure 3-34 plots linear growth rates for the most viscous $\delta=0.2$ experiment. The different $\mathrm{x}$ and $\mathrm{y}$ scales of this plot indicate more anisotropy than earlier examples of f-plane linear growth rates. Here the values of $l$ (the meridional wavenumber) are small, consistent with the north-south appearance of the waves and with the fact that maximum linear growth rates occur when $l=0$. Extra vertical lines are drawn at the wavenumbers corresponding to two waves fitting into the domain. This experiment has few linear growth rate contours outside these lines, meaning that there is little instability for waves with wavenumbers larger than two. This explains why in the most viscous experiment there is essentially only one wave in the domain. (Note that one might question the viability of a homogeneous turbulence model for this case in which there is only one wave in the domain. The reader should keep in mind that this is a process study. When a wide parameter space is covered, understanding is sometimes achieved at the expense of realism.) As friction reduces, more and more scales become unstable (figures 3-35 and 3-36), and 


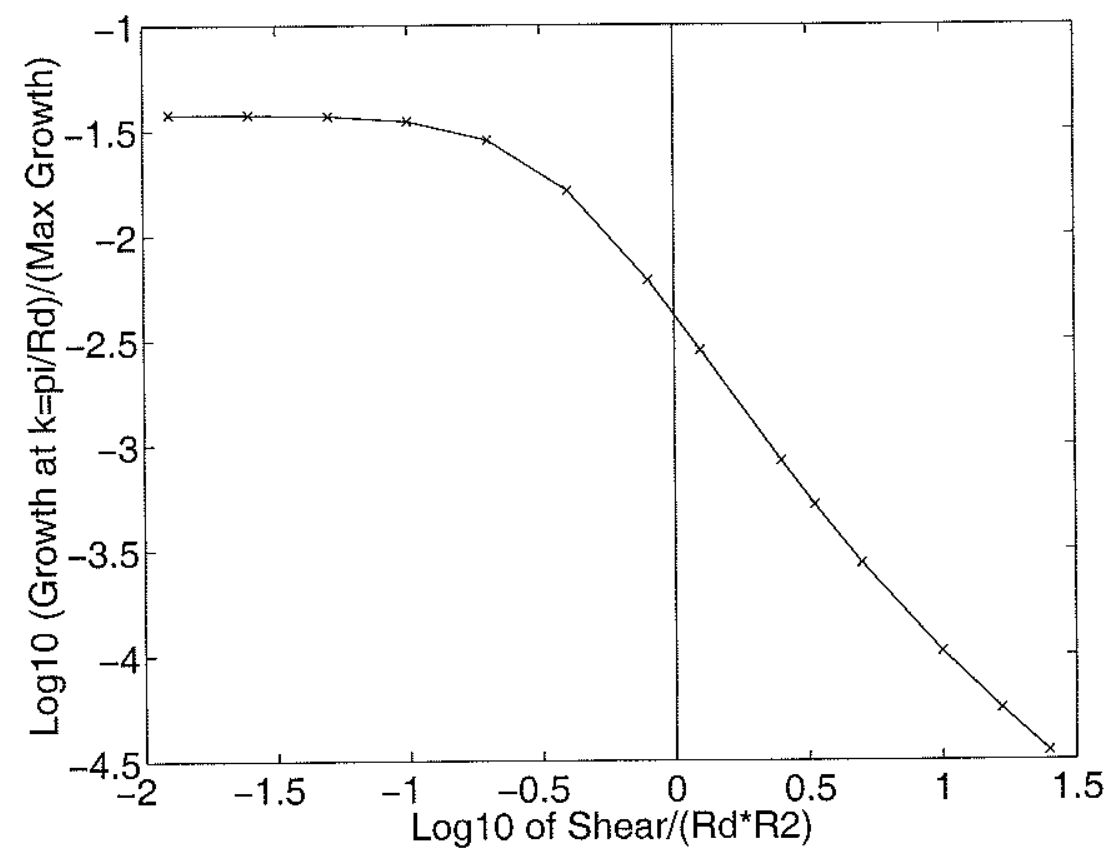

Figure 3-30: Linear growth rates at the length scale $\frac{R_{d}}{\pi}$ divided by the maximum growth rate, as a function of $\frac{\overline{u_{1}-} \overline{u_{2}}}{R_{2} R_{d}}$, for the f-plane $\delta^{\pi}=0.2$ bottom friction only experiments.

the waves become more nonlinear in appearance, until the fields become turbulent at long dissipation times, and ultimately cascade to domain size. Again, the $\delta=1$ experiments are similar in appearance, and are omitted for the sake of brevity. 
159.4 day dissipation

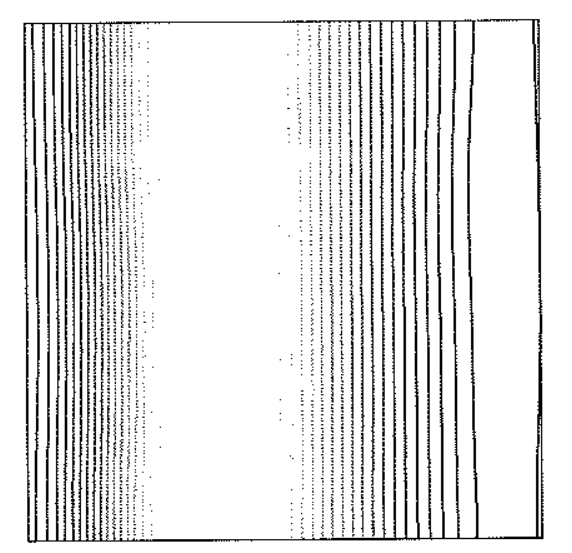

\section{8 day dissipation}

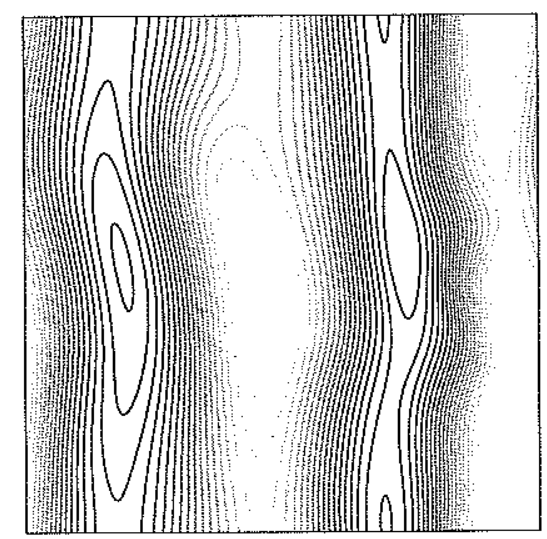

161.2 day dissipation

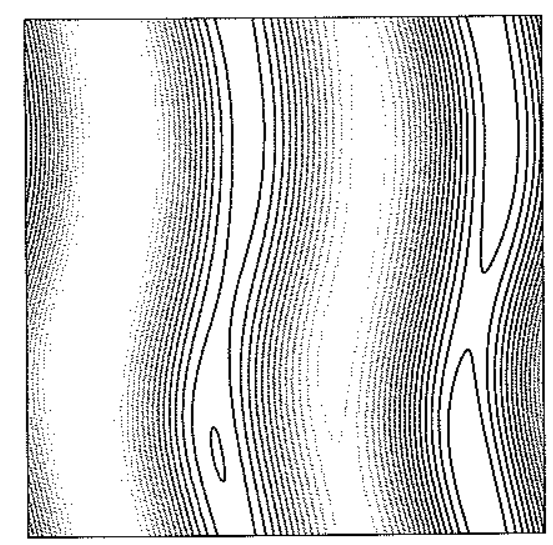

170.7 day dissipation

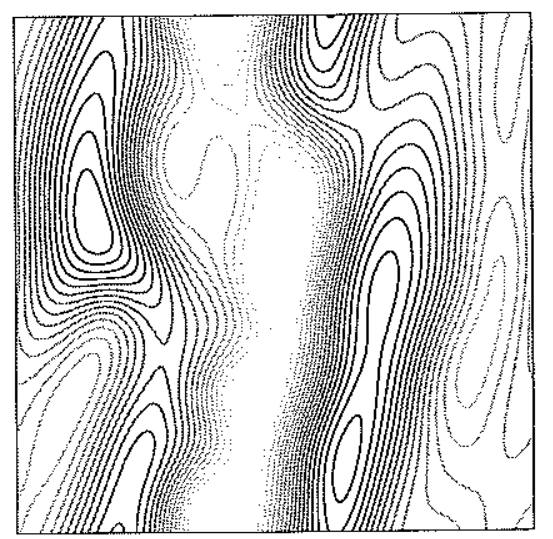

Figure 3-31: Contours of snapshots of top layer fluctuation streamfunction for the symmetric friction $\delta=0.2$ experiments. 


\section{3 day dissipation}

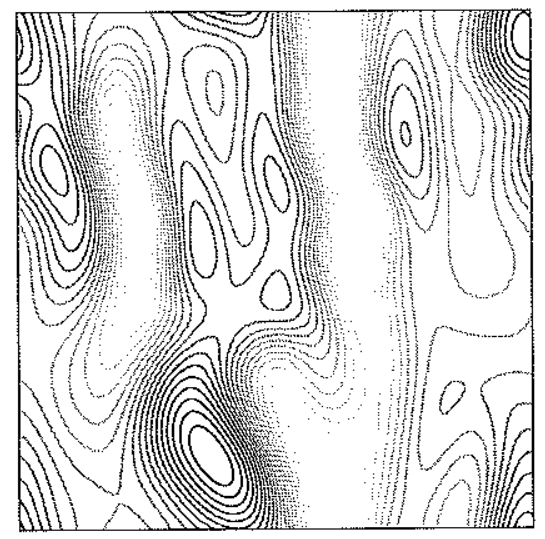

290.2 day dissipation

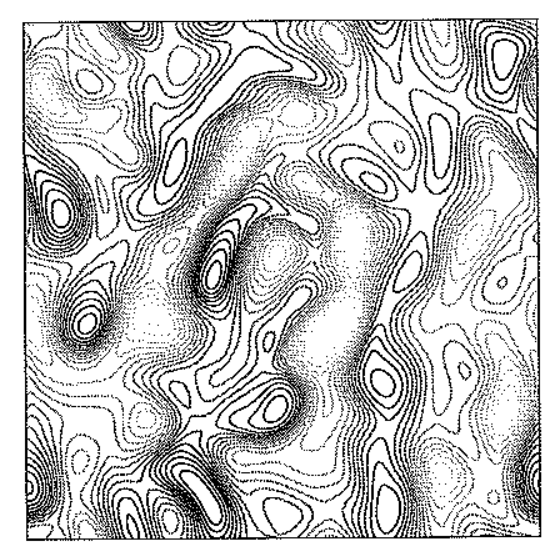

193.4 day dissipation

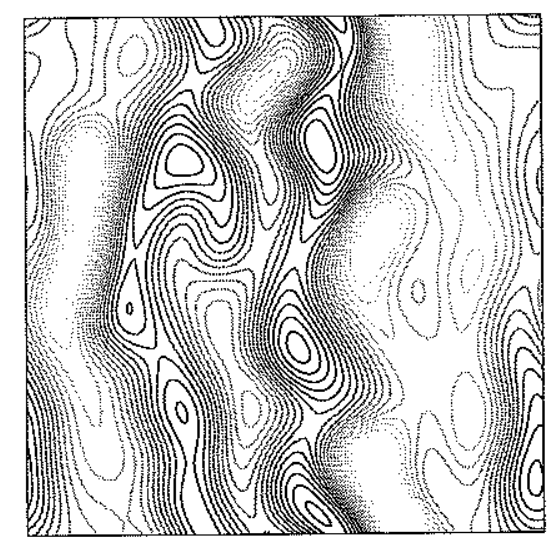

\section{3 day dissipation}

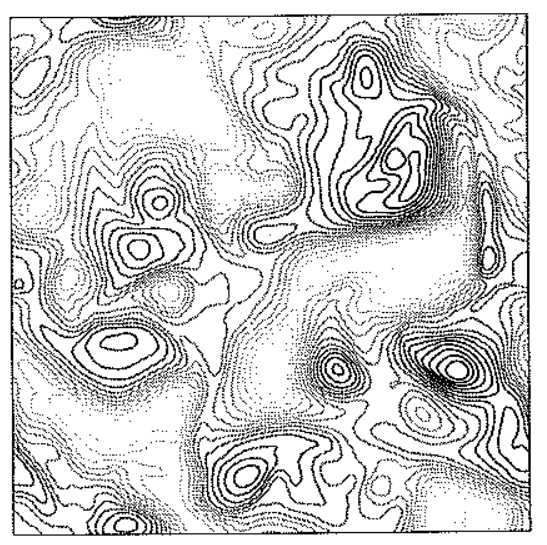

Figure 3-32: Contours of snapshots of top layer fluctuation streamfunction for the symmetric friction $\delta=0.2$ experiments, continued. 
967.2 day dissipation

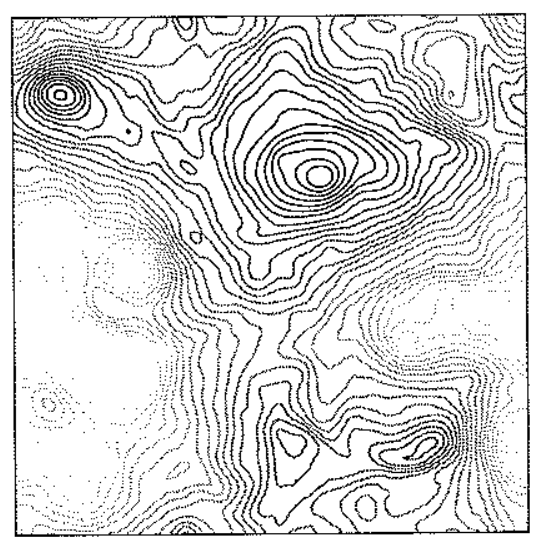

1450.8 day dissipation

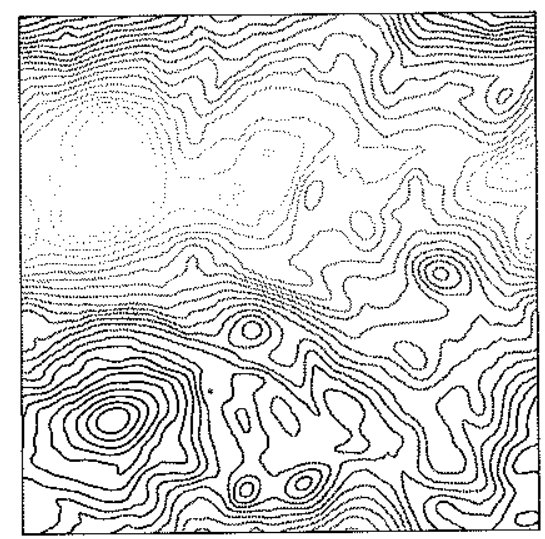

\section{6 day dissipation}

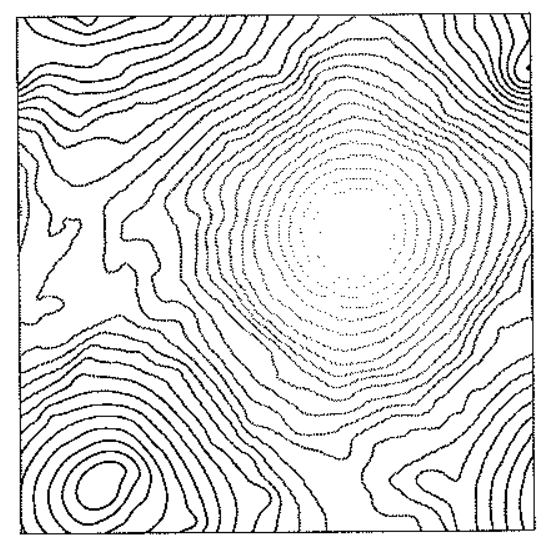

Figure 3-33: Contours of snapshots of top layer fluctuation streamfunction for the symmetric friction $\delta=0.2$ experiments, continued. 


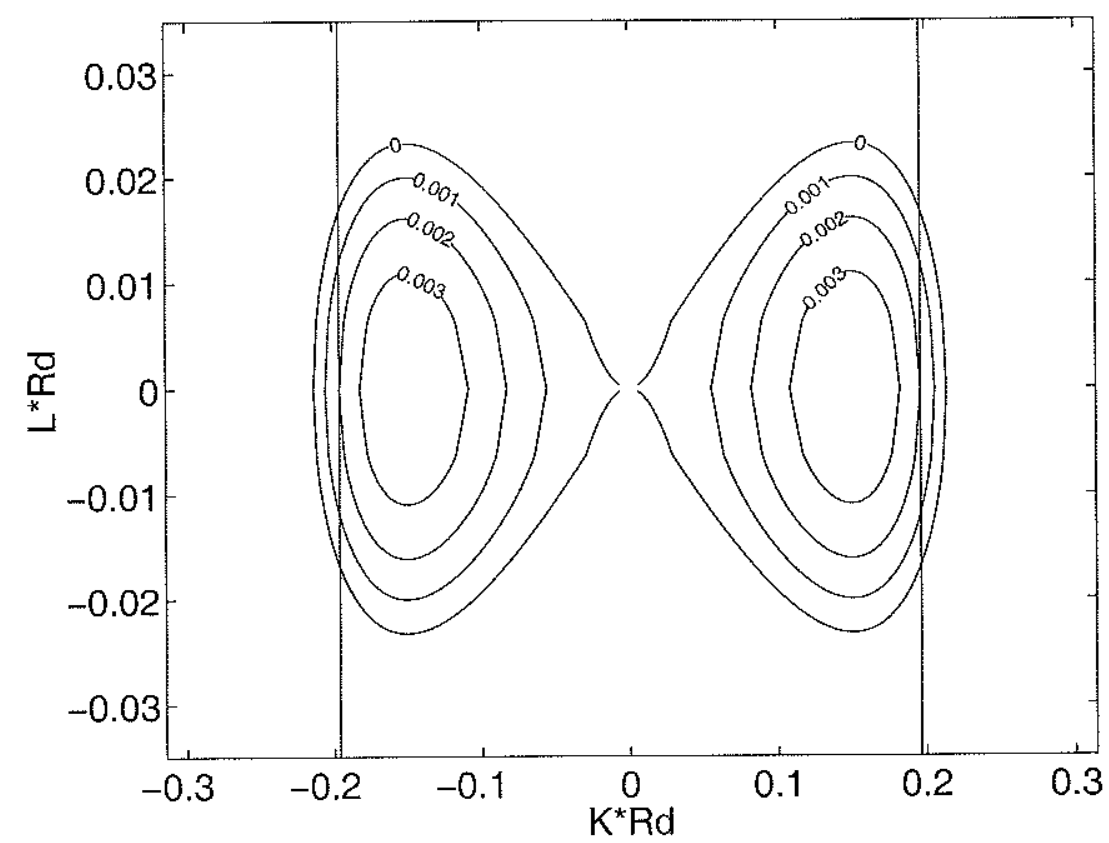

Figure 3-34: Linear growth rates in units of $10^{-8} \mathrm{~s}^{-1}$, for the 159.4 day dissipation time symmetric friction $\delta=0.2 \mathrm{f}$-plane experiment. Extra lines drawn at the scale corresponding to half the domain size.

Modal length scales (measured as reciprocals of appropriate modal energy centroids) are plotted against the ratio of bottom layer nonlinear to Ekman terms in figures 3-37 and 3-38. Length scales of modal kinetic energies for the symmetric friction experiments are also plotted versus dissipation time in figure 3-39. Length scales of baroclinic kinetic energy are always less than or equal to those of available potential energy, because extra derivatives involved in the calculation of the former emphasize smaller scales. Therefore, length scales of total baroclinic energy are always in between those of baroclinic kinetic energy and those of potential energy. At the less viscous end of the parameter range covered, in all four sets of experiments, length scales of baroclinic kinetic energy remain near $R_{d}$, while the barotropic mode cascades nearly to the domain scale when nonlinear terms dominate (since domain size is $20 \pi R_{d}$, eddy lengths cannot exceed $10 R_{d}$ ). Consistent with the barotropic cascade, the length scale of total eddy energy is nearly as large as that of barotropic 

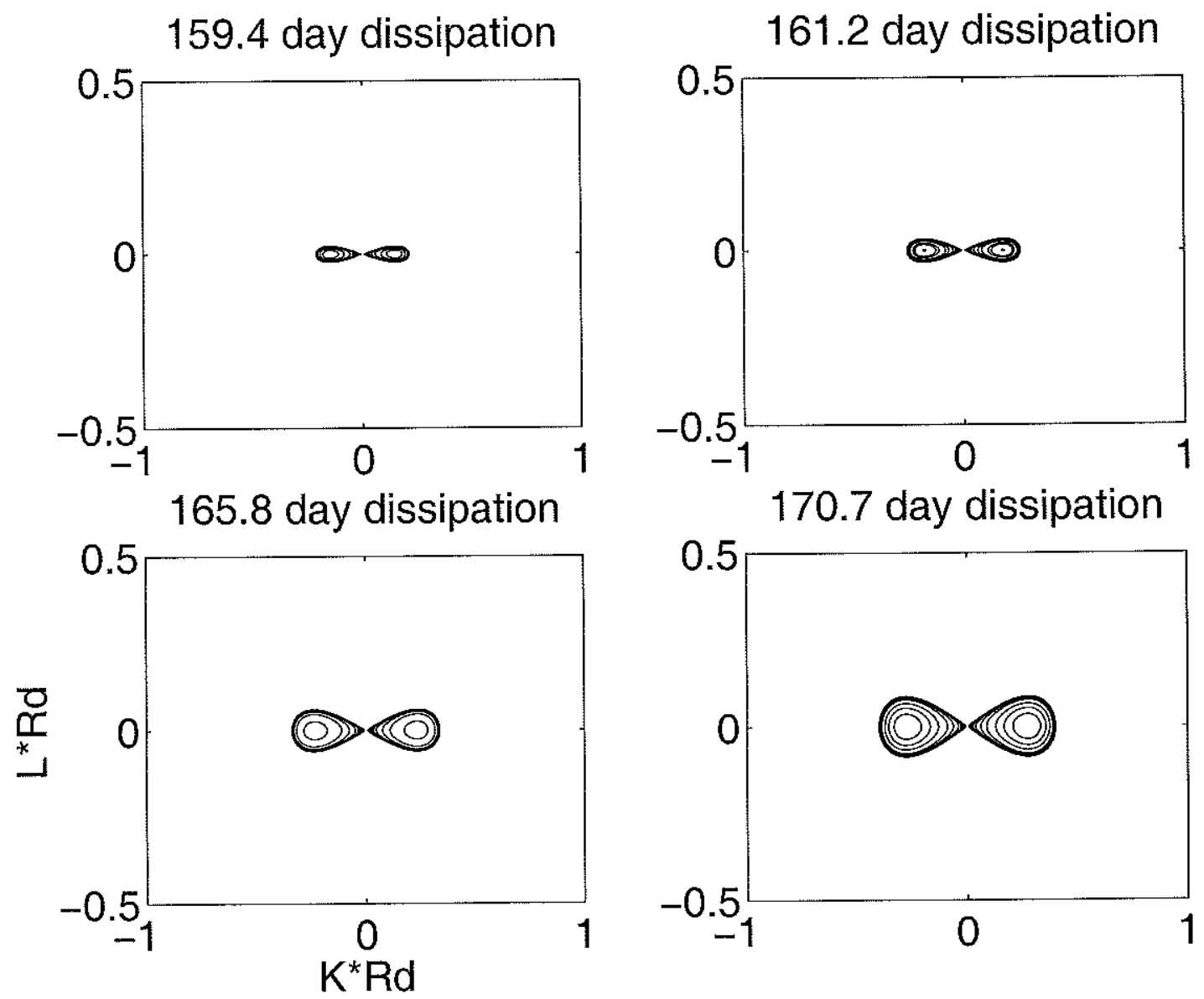

Figure 3-35: Contours of linear growth rates for $\delta=0.2$ symmetric friction experiments. The outermost contour in each plot represents the zero value. The fact that the zero contour extends outwards as friction decreases means that larger wavenumbers (smaller scales) are becoming unstable. 

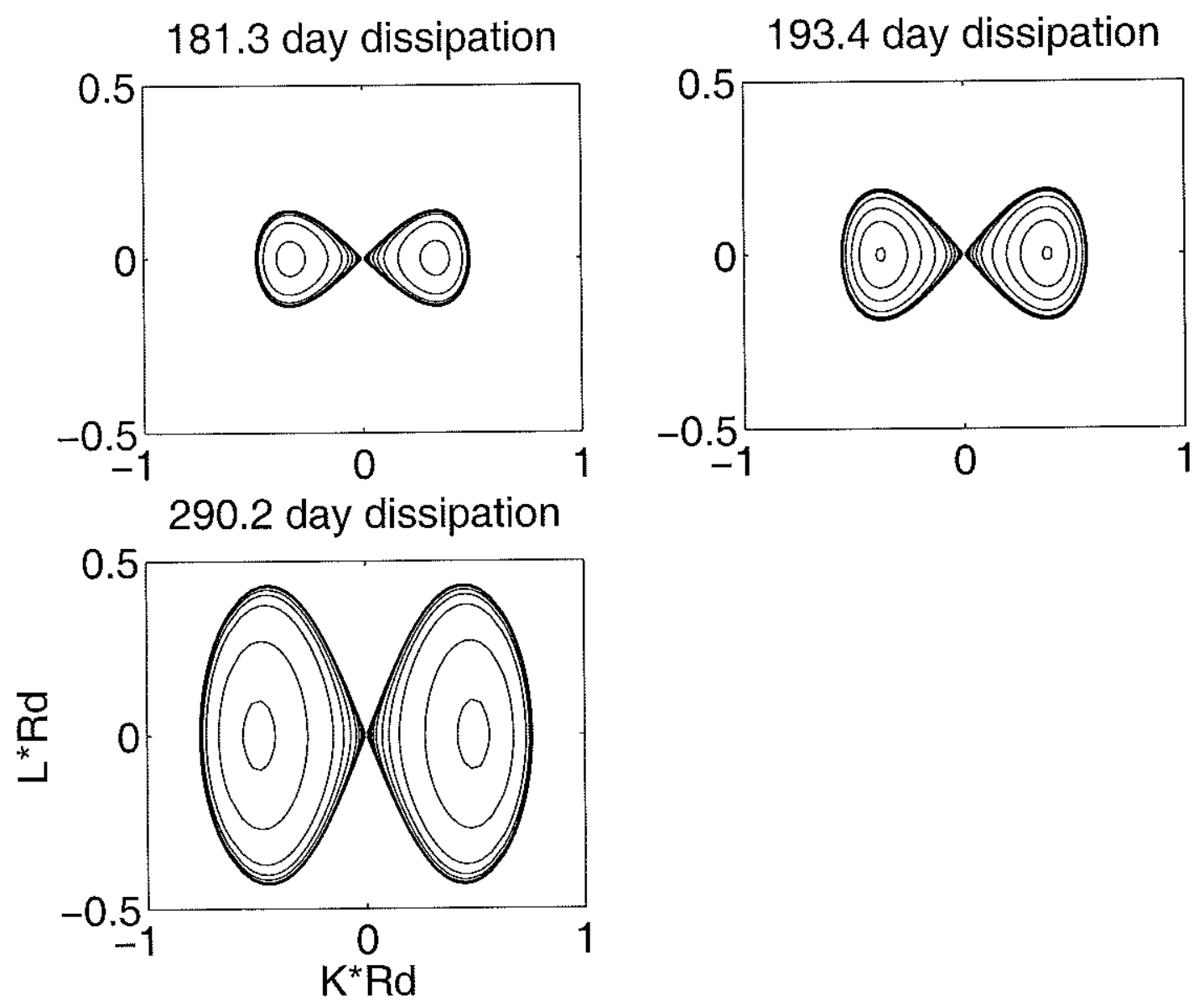

Figure 3-36: Contours of linear growth rates for $\delta=0.2$ symmetric friction experiments. The outermost contour in each plot represents the zero value. 
energy, for the least viscous experiments. At the viscous end of the bottom-friction only plots, length scales associated with the modal kinetic energies are equal, as expected from equation 3.30, and remain near $R_{d}$. In the symmetric friction experiments, eddy length scales become larger as the stability boundary is approached, consistent with the earlier discussions. In the $\delta=0.2$ symmetric friction experiments, the transition between a regime in which nonlinear and Ekman terms are of the same order to one in which the nonlinear term dominates occurs between the 290.2 and 580.3 day dissipation experiments. Figure 3-32 shows that these correspond to the transition between waves and turbulence, and figure 3-38 indicates that this transition also leads to a separation between barotropic and baroclinic kinetic energy length scales.

Length scales of layer kinetic energies are plotted against the ratio of lower layer nonlinear to Ekman terms in figures 3-40 and 3-41. The top layer kinetic energy length scale of the $\delta=0.2290$ day dissipation time bottom friction experiment (our most "realistic" in terms of the ratio of eddy to mean kinetic energy) is 1.26 $R_{d}$. Top layer length scales offer the best opportunity for comparison to altimetry data, since altimeters measure variability of sea surface height. Stammer (1997) did not use centroids as a measure of eddy length scales. Figure 1-2 uses $\frac{1}{2 \pi}$ of the wavelength of maximum surface kinetic energy as a measure. For our most realistic experiment, the wavelength of maximum kinetic energy is at $4 \pi R_{d}$, which corresponds to a length scale $\frac{\lambda}{2 \pi}$ of $2 R_{d}$. Stammer (1997) found that eddy length scales measured this way varied from 2 to $4 R_{d}$ over mid-latitudes. Note that, unlike the $\delta=1$ experiments, even the least viscous of the $\delta=0.2$ experiments have not yet cascaded to domain scale in the top layer. At the less viscous ends of the plots, $\delta=0.2$ experiments (both symmetric friction and bottom friction only) have larger eddies in the bottom layer than in the top layer. This can be explained as follows. Inversion of equations 2.14 and 2.15 yields the following relationships between layer and modal kinetic energies: 

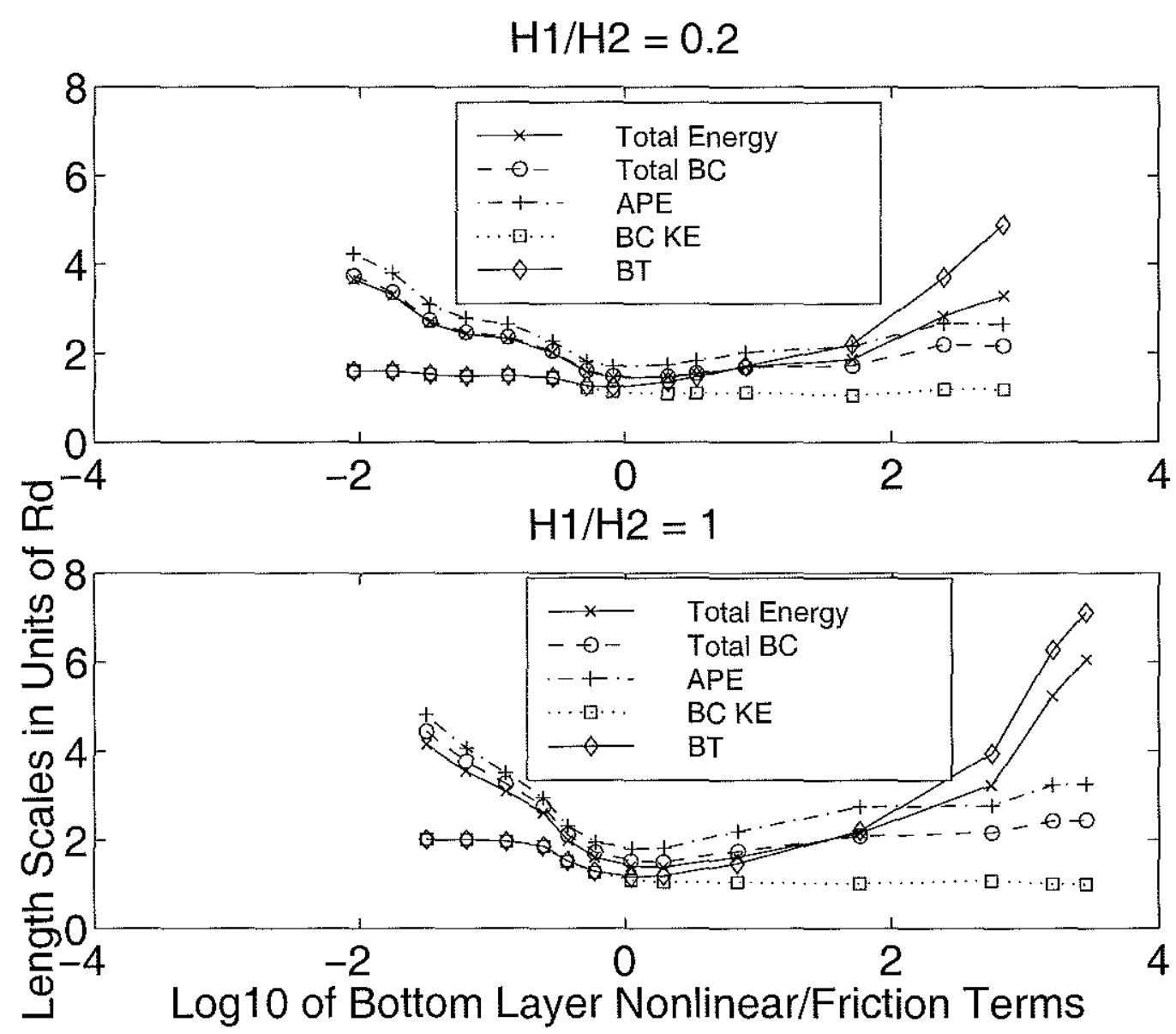

Figure 3-37: Modal eddy length scales, as measured by inverse centroids, for the bottom friction only $\delta=0.2$ (top panel) and $\delta=1$ (bottom panel) experiments, plotted versus $\frac{\delta U_{1} L_{2}}{(1+\delta) R_{2} R_{d}^{2}}$. 

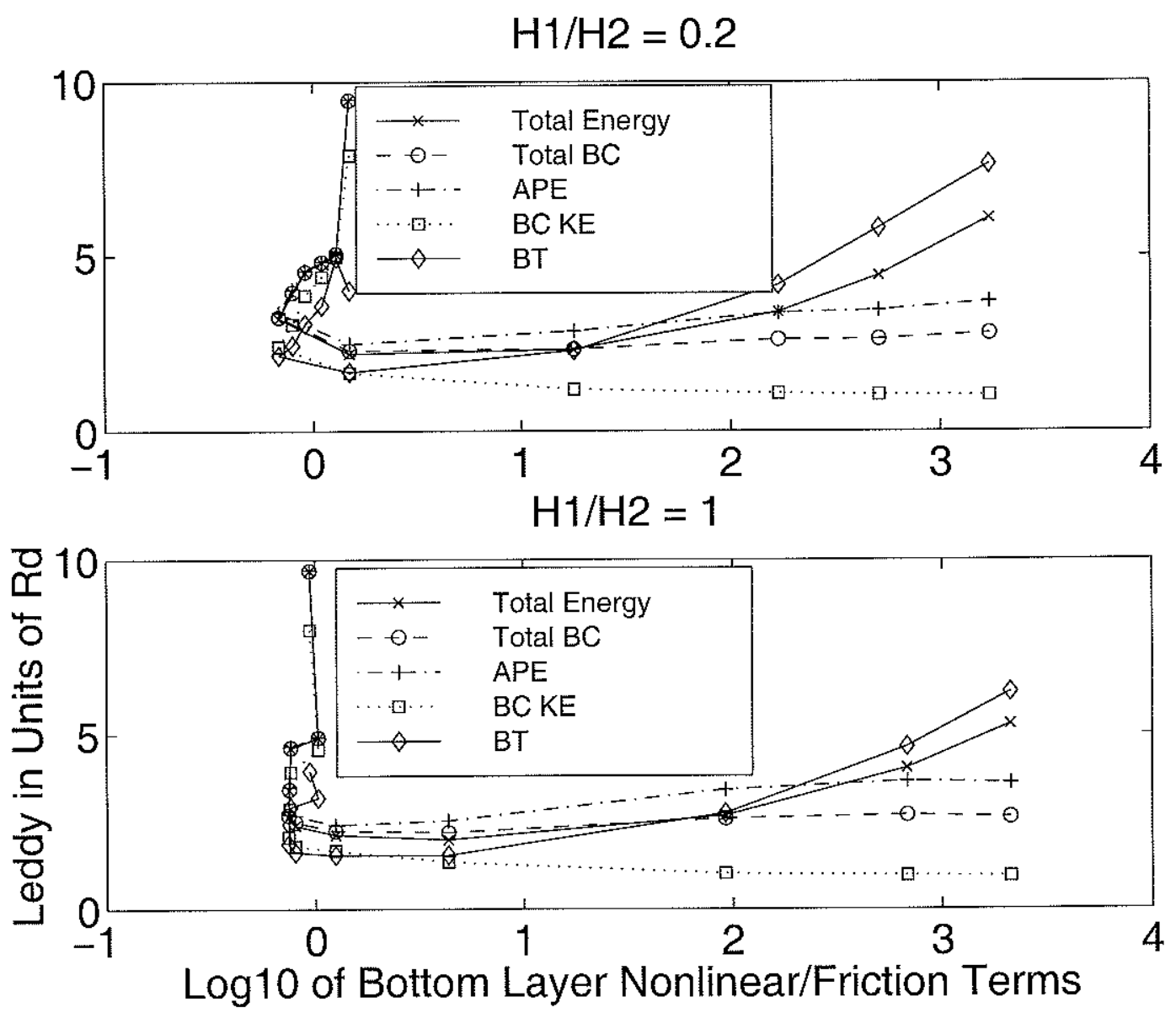

Figure 3-38: Modal eddy length scales, as measured by inverse centroids, for the symmetric friction $\delta=0.2$ (top panel) and $\delta=1$ (bottom panel) experiments, plotted versus $\frac{\delta U_{1} L_{2}}{(1+\delta) R_{2} R_{d l}^{2}}$. 
Delta 0.2
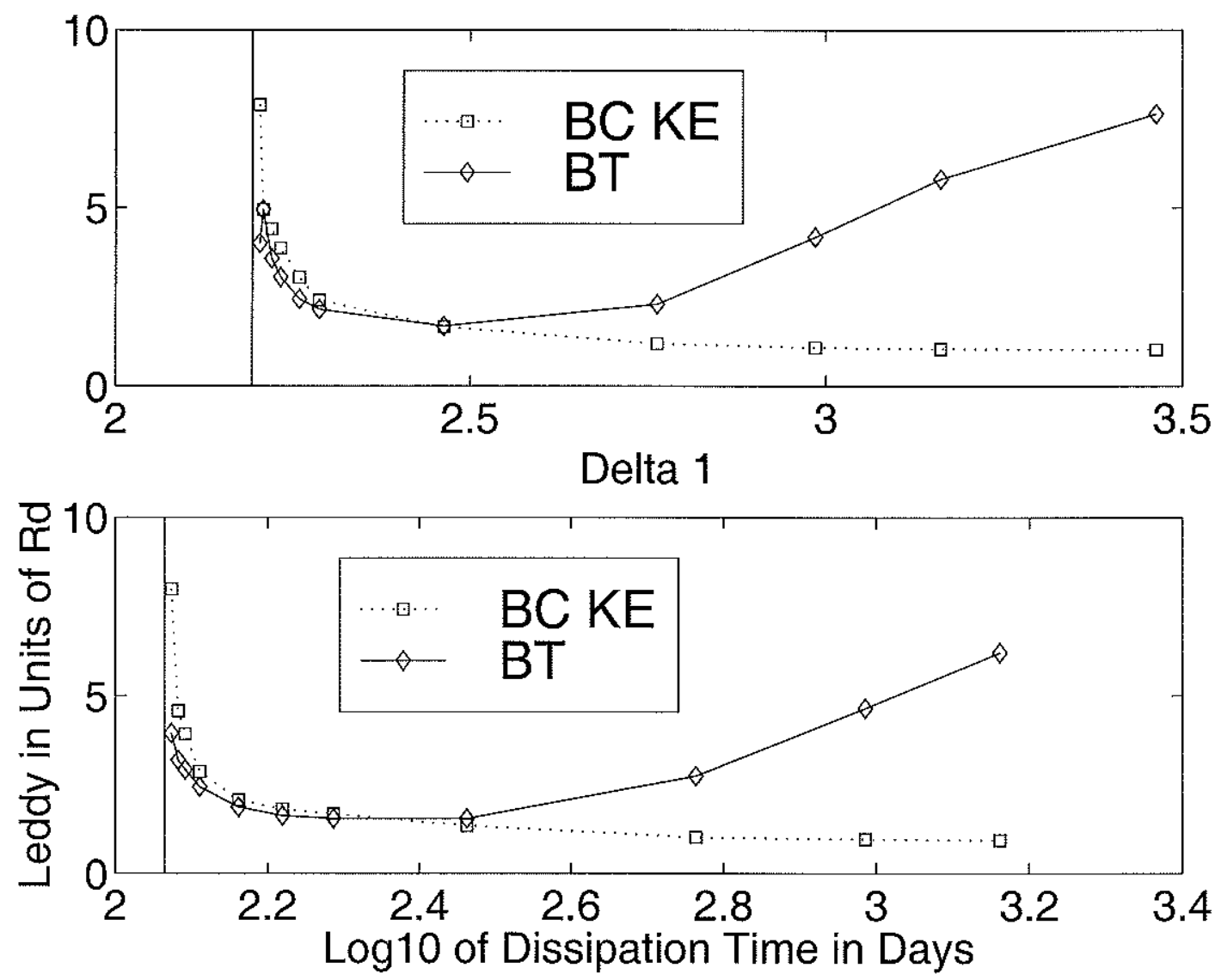

Figure 3-39: Modal eddy length scales, as measured by inverse centroids, for the $\delta=0.2$ (top panel) and $\delta=1$ (bottom panel) symmetric friction experiments, plotted versus dissipation time. 


$$
\begin{aligned}
& \left(\nabla \psi_{1}\right)^{2}=\left(\nabla \psi_{B T}\right)^{2}+\frac{1}{\delta}\left(\nabla \psi_{B C}\right)^{2}+\frac{2}{\sqrt{\delta}} \nabla \psi_{B T} \bullet \nabla \psi_{B C} \\
& \left(\nabla \psi_{2}\right)^{2}=\left(\nabla \psi_{B T}\right)^{2}+\delta\left(\nabla \psi_{B C}\right)^{2}-2 \sqrt{\delta} \nabla \psi_{B T} \bullet \nabla \psi_{B C} .
\end{aligned}
$$

When $\delta=0.2$, the top layer kinetic energy is dominated by the baroclinic mode while the bottom layer kinetic energy is dominated by the barotropic mode. Since the baroclinic mode is at smaller scales in the cascading regime, top layer kinetic energy should therefore be at smaller scales than the bottom layer kinetic energy. There is some evidence for an increase in kinetic energy length scale with depth (though not as much as in these experiments) in eastern North Atlantic current meter records (c.f. page 174 of Mercier and de Verdiere 1985). Barotropic and baroclinic modes contribute equally to the kinetic energies in both top and bottom layers when $\delta=1$. Therefore there should be less difference between the layer scales, as the figures show. Note that the layer scales must be equal in the $\delta=1$ symmetric friction case, as there is nothing to distinguish between layers.

\subsection{Chapter summary}

Freely evolving two dimensional turbulence cascades to large scales. Freely evolving flat-bottom stratified QG turbulence cascades to the barotropic mode and to large scales. Observations of eddy fields in the actual ocean conflict with these model tendencies. Wunsch (1997) showed that the barotropic mode generally accounts for about 35 to 40 percent of the mid-ocean eddy kinetic energy. The rest is taken up by baroclinic modes, predominantly the first baroclinic mode. Since the first baroclinic mode is associated with $R_{d}$, retention of substantial energy in the first baroclinic mode should lead to retention of substantial energy in scales near $R_{d}$.

Stammer (1997) found this to be the case in altimetric data. We have examined 


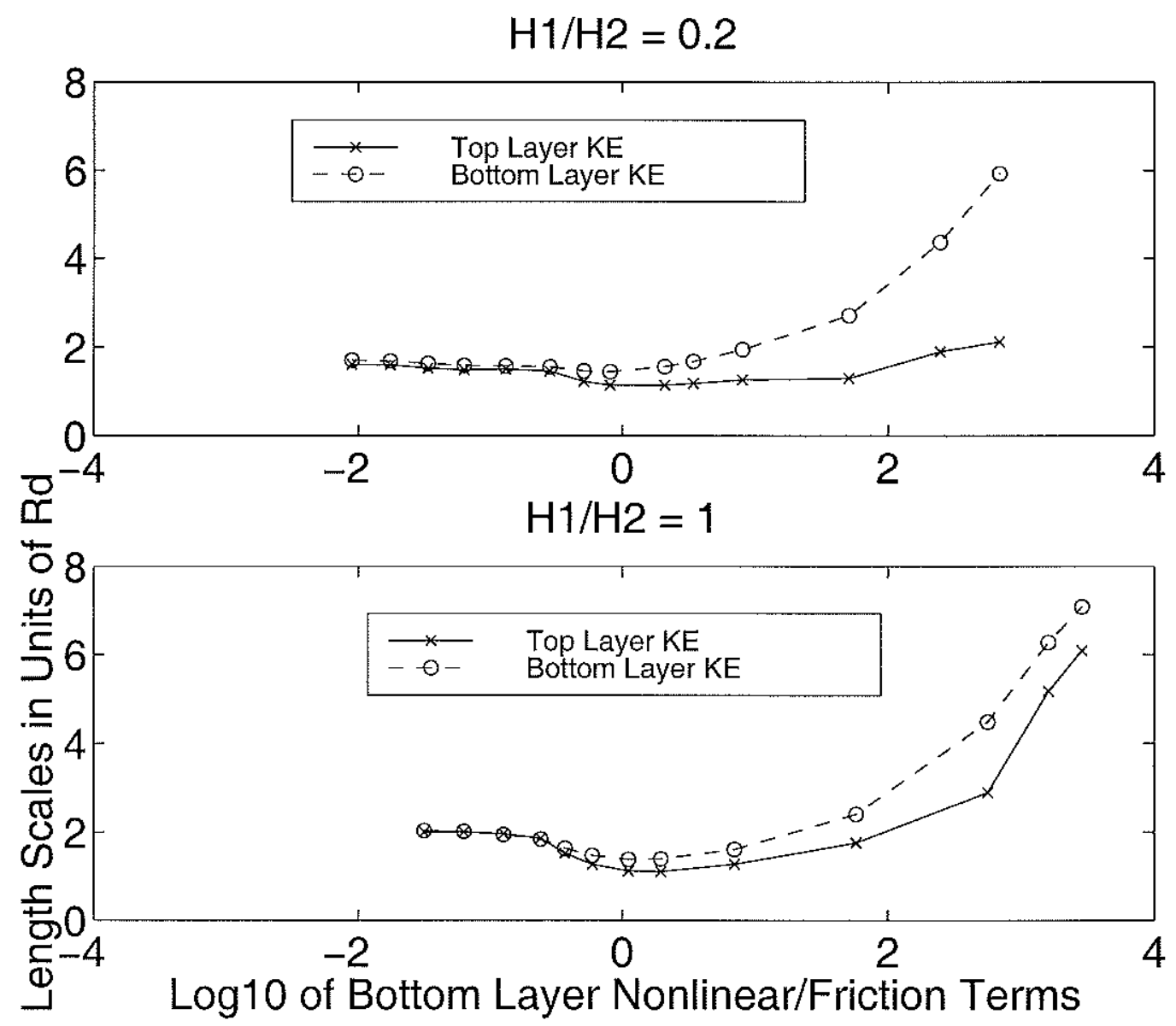

Figure 3-40: Layer eddy length scales, as measured by inverse centroids, for the bottom friction only $\delta=0.2$ (top panel) and $\delta=1$ (bottom panel) experiments, plotted versus $\frac{\delta U_{1} L_{2}}{(1+\delta) R_{2} R_{d}^{2}}$. 


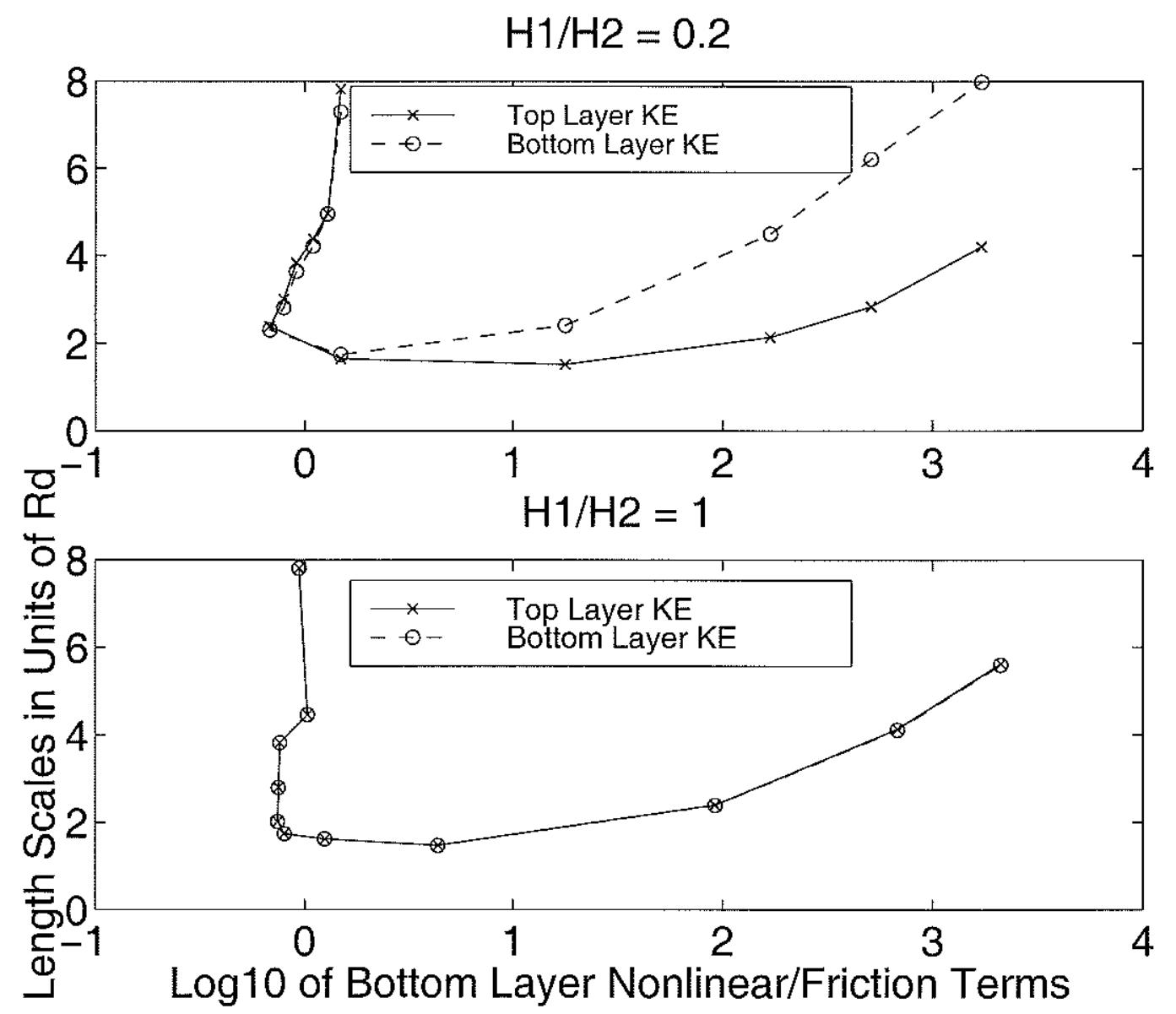

Figure 3-41: Layer eddy length scales, as measured by inverse centroids, for the symmetric friction $\delta=0.2$ (top panel) and $\delta=1$ (bottom panel) experiments, plotted versus $\frac{\delta U_{1} L_{2}}{(1+\delta) R_{2} R_{d}^{2}}$. 
in this chapter how the cascade could be arrested, so that models better match observations.

The central argument of this thesis is that local baroclinic instability of weak mid-ocean gyre flows is a plausible mechanism for generation of mesoscale eddies. A damped, baroclinically unstable flow should hold more energy in the baroclinic mode and in deformation scales. This is because unstable flows continually inject energy into the baroclinic mode and the deformation radius, while friction prevents eddies from cascading far away from this state. However, some influential studies of forceddissipated turbulence, for instance, Salmon (1978), Salmon (1980), and Larichev and Held (1995), found that forced-dissipated model eddy fields still become barotropic and large scale. All of these studies were done in models with two equal layer depths, the analogue of uniform stratification in the continuous case. Here we argue that the level of barotropization should be reduced with surface-trapped stratification and with Ekman friction that is present only in the bottom layer. There are two reasons that this should be so. One is that baroclinic nonlinear self-interactions project onto the baroclinic mode if and only if layer depths are unequal. The other is that the baroclinic mode is weakly damped compared to the barotropic mode when the stratification is surface-trapped and the friction is bottom-trapped.

As a precursor to forced-dissipated experiments, which are our main focus in this thesis, freely evolving experiments on an f-plane were done with two values of the parameter $\delta=\frac{H_{1}}{H_{2}} ; 1$, representing a uniform stratification, and 0.2 , representing a surface-trapped stratification. In both cases, barotropization took place, but it was less complete in the surface-trapped case. This was true whether modal energy ratios or modal enstrophy ratios were used as a measure of eddy baroclinicity. Since there is no Ekman friction in the freely evolving experiments, this difference in behavior must be due to the nonlinear projection mechanism.

Next, eddy baroclinicity in two sets of forced-dissipated experiments having bottom-trapped friction was compared; again, the sets are distinguished from each 
other by their value of $\delta$. When the ratio of lower layer nonlinear to Ekman friction terms is much less than one, lower layer eddy velocities are nearly zero, which implies a strong correlation between modes. This correlation can be used to predict the ratio of baroclinic to barotropic kinetic energies in this regime. The value of the modal ratio in this limiting case serves as an upper bound for the entire set of experiments. When friction weakens so that the nonlinear term dominates over bottom Ekman friction, eddy baroclinicity reduces, consistent with behavior at long times in freely evolving experiments. The upper bound of $\frac{K E_{B C}}{K E_{B T}}$ is one for experiments with $\delta=1$ and 5 for experiments with $\delta=0.2$. Mid-latitude current meter data indicates that the baroclinic mode generally contains about fifty percent more kinetic energy than does the barotropic mode. Therefore the surface-trapped nature of ocean stratification and the bottom-trapped nature of the friction may play an important role in setting observed oceanic eddy baroclinicities. For a fixed amount of total eddy energy, the $\delta=0.2$ experiments retain more baroclinic energy than do the $\delta=1$ experiments, and the enstrophies tell a similar story. Also, $\delta=0.2$ experiments having a realistic ratio of eddy to mean kinetic energy have values of $\frac{K E_{B C}}{K E_{B T}}$ that are near one, which is close to values seen in the mid-ocean. Their length scales compare favorably with observations as well. There are important differences in the dissipation of energy and enstrophy. Ekman friction dominates dissipation of total energy even when it occurs only at the bottom. This is because energy can be transferred from the upper layer to the lower layer and then dissipated by bottom Ekman friction. However, upper layer enstrophy cannot be so transferred and must be dissipated by the small-scale dissipation present in the upper layer. Ekman friction only accounts for a fraction of the total dissipation of enstrophy. This fraction is bounded above by the value it takes in the viscous regime, which can be predicted from a simple argument.

In experiments with bottom friction only, surface-trapped stratification enhances eddy baroclinicity both because of differences in nonlinear modal interactions and 
because the baroclinic mode is weakly damped. More can be discerned about the importance of the two mechanisms from experiments having symmetric Ekman friction. In these experiments, offsets in modal energy ratios are still present when plotted against total eddy energy, but are much reduced. When modal partitioning of energy is the measure of eddy baroclinicity, therefore, the nonlinear projection mechanism seems to be the less important of the two effects. When eddy baroclinicity is measured by modal enstrophy ratios, however, the damping mechanism is less dominant, consistent with the fact that Ekman friction accounts for only a fraction of the enstrophy dissipated in bottom-friction experiments.

Plots of upper layer streamfunction of the model eddy fields display more differences between bottom-friction only experiments and symmetric friction experiments. The latter experience a cutoff value of Ekman friction which can be obtained from a linear stability analysis, consistent with Barcilon (1964). As friction increases beyond this value, eddies disappear. The model exists in a regime of long, weakly nonlinear waves near the stability boundary. As friction decreases away from the cutoff value, linear stability analysis indicates that smaller scales become unstable. Consistent with this, eddy length scales decrease, and the waves take on a more nonlinear appearance, before breaking up into a smaller scale, turbulent eddy field. As friction reduces still more, eddies cascade to larger scales. The bottom friction experiments are noticably less wavelike for high values of friction, which, no matter how large it becomes, does not stabilize the eddy field. The appearance of eddies in this viscous regime is quite unlike that seen in previous f-plane forced-dissipated studies. Small-scale fronts are noticable, and may be related to the destabilization of short waves caused by large values of bottom friction, as argued by Williams and Robinson (1974). As friction reduces, the fronts break up into eddies, and a cascade to large scales takes place.

When nonlinear terms dominate over Ekman friction in the bottom layer, length scales associated with modal kinetic energies separate. The baroclinic scale remains 
near $R_{d}$, while the barotropic scale cascades to nearly domain size. Bottom layer length scales tend to be larger than upper layer length scales in the presence of surface-trapped stratification, consistent with a simple argument and with a limited number of observations. Surface-trapped stratification enhances the baroclinic mode, and increases the ratio of baroclinic to barotropic kinetic energy in the upper layer, enabling top layer length scales to resist the cascade to large scales more effectively than their uniform stratification counterparts.

In the next chapter a mathematical tool will be derived which ties together much of what was presented in this chapter; the baroclinic instability mechanism, the importance of surface-trapped stratification, and the importance of the bottomtrapped nature of friction. Application of this tool to the experiments performed in the present chapter will inform us quantitatively about the importance of these mechanisms in arresting barotropization and the inverse cascade. 


\section{Chapter 4}

\section{Quantification of the inverse}

\section{cascade to large scales and the}

\section{barotropic mode with a cascade}

\section{inequality}

In this chapter we seek a quantitative explanation of eddy length scales for the forced-dissipated experiments presented in the last chapter. One might ask for a theory that dictates under what conditions cascades take place to scales larger than the forcing scale of a baroclinically unstable flow-namely, the deformation radiusand under what conditions the cascade may be halted. Looked at in another way, one might ask whether the energy and enstrophy equations can be manipulated in the forced-dissipated case in a manner similar to the way Fjortoft (1953) manipulated them in the freely evolving case.

Glenn Flierl has indeed produced such an argument, in the case of a one layer flow forced at a particular scale and dissipated through Ekman friction; this could apply, for instance, to the work of Maltrud and Vallis (1991). The argument comes in the form of an inequality which states that energy containing scales at equilibration 
will be larger than the forcing scale. Although the one layer inequality is never used in this dissertation, its derivation is given below for illustrative purposes. It will then be generalized to a damped two-layer baroclinically unstable system. When applied to the results of the preceding chapter, the two-layer inequality elucidates the effects of friction and stratification on the inverse cascade to scales larger than the deformation radius and to the barotropic mode.

\subsection{Derivation of one-layer cascade inequality}

The governing equation for a one-layer forced-dissipated system is:

$$
\frac{\partial}{\partial t} \nabla^{2} \psi+J\left(\psi, \nabla^{2} \psi\right)=-R \nabla^{2} \psi+\text { forcing }
$$

The forcing is assumed to be confined to a narrow band in wavenumber space, so that there is a well defined forcing scale. The energy and enstrophy equations are obtained through multiplication of 4.1 by $\psi$ and $\nabla^{2} \psi$, respectively, and integration over the domain:

$$
\begin{gathered}
\frac{\partial}{\partial t} K E=-2 R K E-\iint \psi \text { forcing } d x d y \\
\frac{\partial}{\partial t} Z=-2 R Z-k_{F}^{2} \iint \psi \text { forcing } d x d y
\end{gathered}
$$

where $k_{F}$ is the scale of the forcing and:

$$
\begin{gathered}
K E=\iint(\nabla \psi)^{2} d x d y=\int E(k) d k \\
Z=\iint\left(\nabla^{2} \psi\right)^{2} d x d y=\int k^{2} E(k) d k
\end{gathered}
$$


In a statistically steady state the $\frac{\partial}{\partial t}$ terms drop out of time averages of 4.2 and 4.3 , and the forcing terms can be eliminated, leaving:

$$
\left[\int k^{2} E(k) d k\right]=k_{F}^{2}\left[\int E(k) d k\right]
$$

where brackets denote time averaging. Let $k_{E}=\frac{\int k E(k) d k}{\int E(k) d k}$. Begin with the selfevident inequality:

$$
\left[\int\left(k-k_{E}\right)^{2} E(k) d k\right] \geq 0
$$

which can be manipulated to give:

$$
\left[\int k^{2} E(k) d k\right] \geq\left[k_{E}^{2} \int E(k) d k\right]
$$

which, together with 4.6 , yields:

$$
k_{F}^{2} \geq \frac{\left[k_{E}^{2} \int E(k) d k\right]}{\left[\int E(k) d k\right]}
$$

We have shown that energy cascades beyond the scales at which it is injected in an equilibrated, forced-dissipated flow. This has been argued to be the case in many papers (c.f. Kraichnan 1967; Leith 1968; Batchelor 1969; Salmon 1978; Salmon 1980; Maltrud and Vallis 1991; Larichev and Held 1995). We are able to explicitly prove it in this case. Note that dissipation of energy (enstrophy) by Ekman friction is proportional to energy (enstrophy) itself, enabling the argument to be made easily. Note also (Geoffrey Vallis, personal communication) that the nonlinear term $J\left(\psi, \nabla^{2} \psi\right)$ does not participate in the derivation, because it does not contribute to generation of domain-integrated energy or enstrophy. The inequality would be unaltered in a system governed by equation 4.1 but with the Jacobian 
term set to zero. Since scale transfer is effected by nonlinear terms, one senses an apparent contradiction. The argument is rescued by the fact that 4.9 is not a strict inequality. If a linear system were forced at a scale $k_{F}$, energy would remain at that scale and 4.9 would be an equality. Note that small scale dissipation could be included in this argument, and 4.9 would be unchanged.

Although developed independently by Glenn Flierl, and not widely known in the community, this type of argument has apparently been made by others (Andrew Majda, personal communication). According to Majda, it can be extended to include different types of friction, and may have been extended to two layers by others. This author is not aware of any other studies which make extensive use of the two-layer inequality in interpreting numerical results, as will be done here.

\subsection{Derivation of two-layer cascade inequality}

We now generalize the one-layer result to the two-layer damped baroclinically unstable system relevant to this thesis. By analogy to the one layer case, the forcing terms (which are now in the form of eddy PV fluxes multiplied by mean shears) can be eliminated from the modal energy and enstrophy equations 2.29 and 2.30. We do so by multiplying the latter by $-R_{d}^{2}$ and then adding it to the former, resulting in:

$$
\begin{gathered}
\frac{\partial}{\partial t} \iint \frac{1}{2}\left[\left(\nabla \psi_{B T}\right)^{2}+\left(\nabla \psi_{B C}\right)^{2}+\frac{1}{R_{d}^{2}} \psi_{B C}^{2}-R_{d}^{2}\left(q_{B T}^{2}+q_{B C}^{2}\right)\right] d x d y \\
=-\left(\frac{\delta R_{1}+R_{2}}{1+\delta}\right) \iint\left(\nabla \psi_{B T}\right)^{2} d x d y \\
+R_{d}^{2}\left(\frac{\delta R_{1}+R_{2}}{1+\delta}\right) \iint\left(\nabla^{2} \psi_{B T}\right)^{2} d x d y+R_{d}^{2}\left(\frac{R_{1}+\delta R_{2}}{1+\delta}\right) \iint\left(\nabla^{2} \psi_{B C}\right)^{2} d x d y
\end{gathered}
$$




$$
+\frac{\sqrt{\delta}\left(R_{2}-R_{1}\right)}{1+\delta} \iint\left(\nabla \psi_{B T} \bullet \nabla \psi_{B C}-2 R_{d}^{2} \nabla^{2} \psi_{B T} \nabla^{2} \psi_{B C}\right) d x d y
$$

Now define centroids of the modal kinetic energy spectra:

$$
\begin{aligned}
k_{B T} & =\frac{\int k E_{B T}(k) d k}{\int E_{B T}(k) d k} \\
k_{B C} & =\frac{\int k E_{B C}(k) d k}{\int E_{B C}(k) d k} .
\end{aligned}
$$

We take a time average of 4.10 and write the enstrophies spectrally:

$$
\begin{gathered}
{\left[K E_{B T}\right]=R_{d}^{2}\left[\int k^{2} E_{B T}(k) d k\right]+R_{d}^{2}\left(\frac{R_{1}+\delta R_{2}}{\delta R_{1}+R_{2}}\right)\left[\int k^{2} E_{B C}(k) d k\right]} \\
+\frac{\sqrt{\delta}\left(R_{2}-R_{1}\right)}{2\left(\delta R_{1}+R_{2}\right)}\left[\iint\left(\nabla \psi_{B T} \bullet \nabla \psi_{B C}-2 R_{d}^{2} \nabla^{2} \psi_{B T} \nabla^{2} \psi_{B C}\right) d x d y\right] .
\end{gathered}
$$

We now apply analogs of 4.8 to each of the modes separately:

$$
\begin{aligned}
& {\left[\int k^{2} E_{B T}(k) d k\right] \geq\left[k_{B T}^{2} \int E_{B T}(k) d k\right]} \\
& {\left[\int k^{2} E_{B C}(k) d k\right] \geq\left[k_{B C}^{2} \int E_{B C}(k) d k\right] .}
\end{aligned}
$$

Combining these with 4.13 yields:

$$
\begin{gathered}
1 \geq R_{d}^{2} \frac{\left[k_{B T}^{2} K E_{B T}+\frac{R_{1}+\delta R_{2}}{\delta R_{1}+R_{2}} k_{B C}^{2} K E_{B C}\right]}{\left[K E_{B T}\right]} \\
+\sqrt{\delta}\left(\frac{R_{2}-R_{1}}{\delta R_{1}+R_{2}}\right) \frac{\left[\iint\left(\nabla \psi_{B T} \bullet \nabla \psi_{B C}-2 R_{d}^{2} \nabla^{2} \psi_{B T} \nabla^{2} \psi_{B C}\right) d x d y\right]}{2\left[K E_{B T}\right]}
\end{gathered}
$$


Again, brackets denote a time average. The deformation radius takes the place of $k_{F}$ as the forcing scale, as one would expect in a baroclinically unstable system. The simplest form of the inequality occurs when $R_{1}=R_{2}$, in which case 4.15 reduces to:

$$
1 \geq R_{d}^{2} \frac{\left[k_{B T}^{2} K E_{B T}+k_{B C}^{2} K E_{B C}\right]}{\left[K E_{B T}\right]} .
$$

If we ignore the time-averaging operator, this roughly tells us that:

$$
1 \geq R_{d}^{2}\left(k_{B T}^{2}+k_{B C}^{2} \frac{K E_{B C}}{K E_{B T}}\right) .
$$

When frictional spin-down time is equal in the two layers, the fluid behaves "twodimensionally", in the sense that barotropic, large-scale motions are preferred. It cannot simultaneously retain substantial energy in length scales near $R_{d}$ and in the baroclinic mode. This will be tested in the next section.

\subsection{Evaluation of two-layer cascade inequality in symmetric friction case}

Figures 4-1 and 4-2 display the evaluation of the cascade inequality versus dissipation time and versus the ratio of bottom layer nonlinear to Ekman terms, for the symmetric friction experiments. The quantity on the right-hand side of equation 4.16, which we call the "two-dimensionality measure", is indeed bounded by one. The measure approaches one near the stability boundary, when nonlinear and Ekman terms are of the same order. A cascade to the barotropic mode and to large scales ensues, and the measure becomes significantly less than one, when the nonlinear term becomes larger than the Ekman term. The $\delta=0.2,161.2$ day dissipation time experiment comes closest, at 0.995 , to breaking the inequality. As discussed in chapter 2, it was found that when Ekman friction is present in both 
layers and sufficiently large, the wavenumber filter could be turned off. The six most viscous $\delta=0.2$ symmetric friction experiments, and the seven most viscous $\delta=1$ symmetric friction experiments, were run without a wavenumber filter. This was done in order to match conditions in the numerical experiment as closely as possible to those assumed in the development of the cascade inequality, which ignored the effect of the filter on enstrophy dissipation at small scales. (However, some amount of small-scale filtering is unavoidable in a spectral model. For instance, our method of de-aliasing is to truncate energy at scales smaller than the Nyquist scale.)

The cascade inequality allows us to make connections between the separate behaviors of eddy baroclinicities and length scales. The behavior of eddy length scales near the stability boundary (figure 3-39) was qualitatively explained in chapter 3, from the friction-induced changes in unstable scales. Near the stability boundary, eddy fields are wavelike in appearance, not turbulent. Therefore, nonlinear cascades are not fully developed, which is why the two-dimensionality measure is close to one, rather than much less one, near the stability boundary. We can therefore use the cascade inequality and the length scales (which we take as "explained" from the linear stability results) to predict eddy baroclinicities near the stability boundary. Near the boundary length scales are much larger than $R_{d}$. Therefore the ratio $\frac{K E_{B C}}{K E_{B T}}$ must be much larger than one in order for the two-dimensionality measure to remain near one. In turn this means that $k_{B C}^{2} \frac{K E_{B C}}{K E_{B T}}$ will dominate the two-dimensionality measure, and 4.16 can be approximated by $1 \geq R_{d}^{2} k_{B C}^{2}\left[\frac{\left[K E_{B C}\right]}{\left[K E_{B T}\right]}\right.$, or:

$$
\frac{L_{B C}^{2}}{R_{d}^{2}} \geq \frac{\left[K E_{B C}\right]}{\left[K E_{B T}\right]}
$$

where $L_{B C}$ is the reciprocal of $k_{B C}$. Figure 4-3 shows the predicted upper bound on the baroclinicity ratio $\frac{L_{B C}^{2}}{R_{d}^{2}}$, as well as the actual baroclinicity ratio taken by the experiments, as a function of the ratio of bottom layer nonlinear to Ekman terms. When the latter ratio is order one, the predicted and actual values of $\frac{\left[K E_{B C}\right]}{\left[K E_{B T}\right]}$ are quite similar. When the nonlinear term begins to dominate over friction, the baroclinicity 

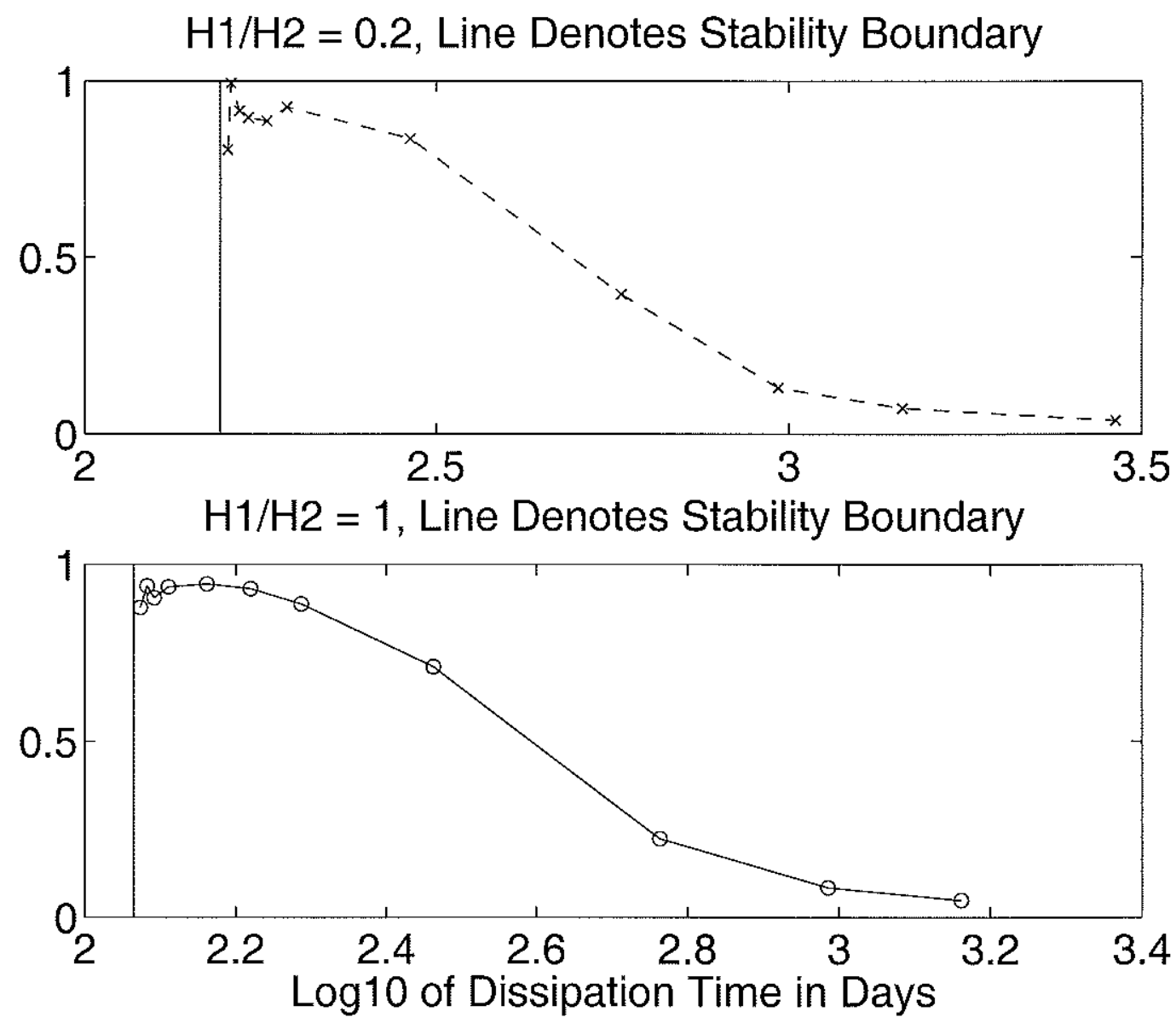

Figure 4-1: Evaluation of the two-dimensionality measure $\frac{\left[k_{B T}^{2} K E_{B T}+k_{B C}^{2} K E_{B C}\right]}{\left[K E_{B T}\right]}$ for the 256 squared symmetric friction experiments, plotted against dissipation time. The measure is bounded by one for all experiments, supporting the analytical work. 

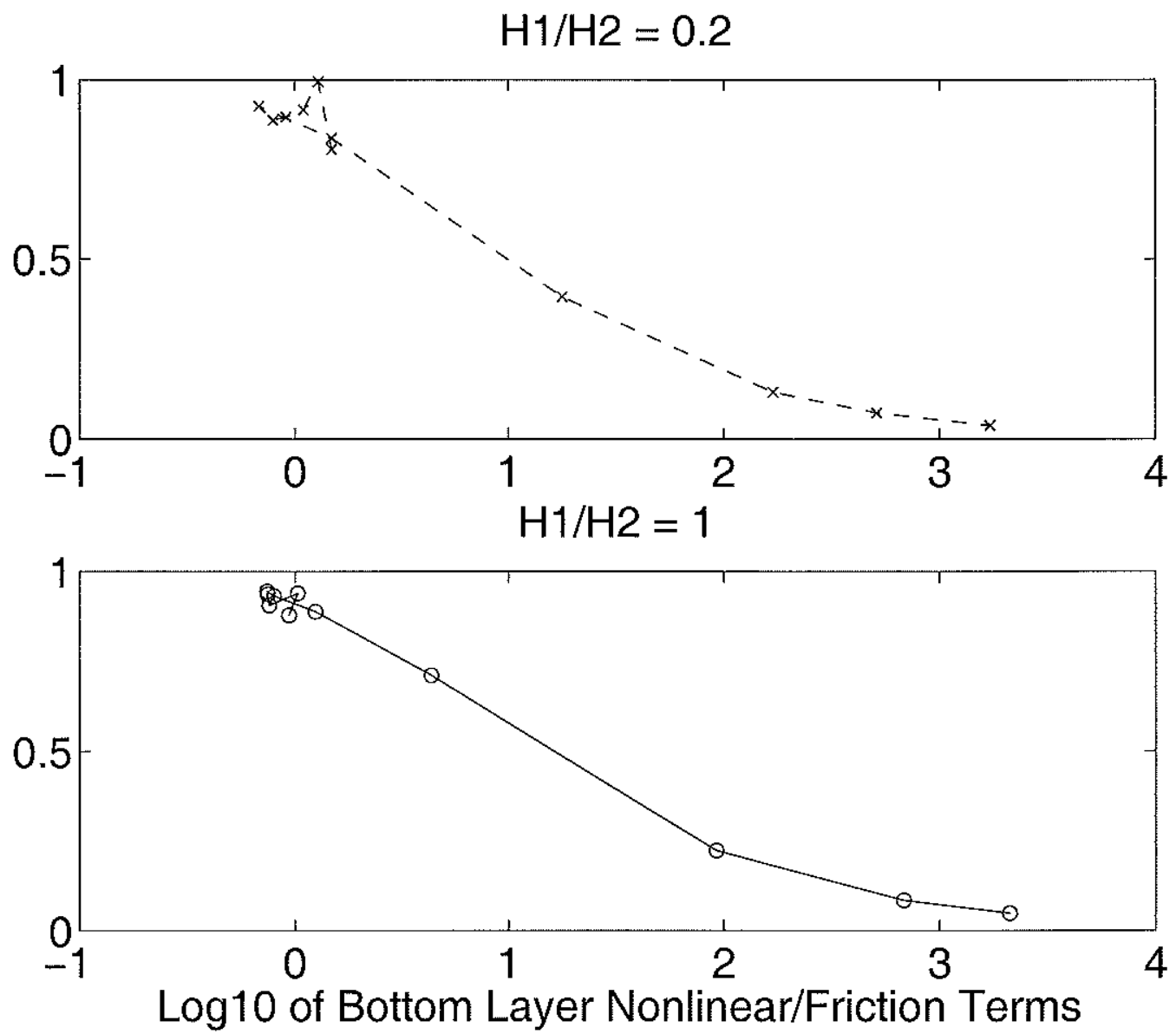

Figure 4-2: Evaluation of the two-dimensionality measure $\frac{\left[k_{B T}^{2} K E_{B T}+k_{B C}^{2} K E_{B C}\right]}{\left[K E_{B T}\right]}$ for the 256 squared symmetric friction experiments, plotted against $\frac{\delta U_{1} L_{2}}{(1+\delta) R_{2} R_{d}^{2}}$. The measure is near one for experiments in which nonlinear and Ekman terms are nearly equal. It becomes substantially less than one when the nonlinear term is much larger than friction. 
ratio becomes substantially less than the predicted value. Note also that because the baroclinic length scale cannot exceed $10 R_{d}$ (the domain size divided by $2 \pi$ ), $k_{B C}^{2} R_{d}^{2}$ cannot be less than $\frac{1}{100}$, meaning that the baroclinicity ratio cannot exceed 100. Figure 3-23 indicates that this is the case, though the baroclinicity ratios have not yet closed in on the upper bound very closely.

When the ratio of nonlinear to Ekman terms exceeds one, a cascade to the barotropic mode, and to large scales in the barotropic mode, proceeds, meaning that the two-dimensionality measure becomes significantly less than one. In the symmetric friction case, eddies cannot simultaneously contain substantial energy in the baroclinic mode and in scales near $R_{d}$. Yet in the actual ocean eddies are strongly baroclinic and compact. We will see in the next section that vertically asymmetric (bottom) Ekman friction, especially when coupled with surface-trapped stratification, allows eddies to behave less like a two-dimensional fluid, in other words, to achieve baroclinicity and compactness at the same time.

\subsection{Evaluation of two-layer cascade inequality in bottom friction only case}

When Ekman friction occurs in the bottom layer only $\left(R_{1}=0\right)$, the cascade inequality 4.15 becomes more complicated than in the symmetric friction case:

$$
\begin{gathered}
1 \geq R_{d}^{2} \frac{\left[k_{B T}^{2} K E_{B T}+\frac{H_{1}}{H_{2}} k_{B C}^{2} K E_{B C}\right]}{\left[K E_{B T}\right]} \\
+\sqrt{\delta} \frac{\left[\iint\left(\nabla \psi_{B T} \bullet \nabla \psi_{B C}-2 R_{d}^{2} \nabla^{2} \psi_{B T} \nabla^{2} \psi_{B C}\right) d x d y\right]}{2\left[K E_{B T}\right]}
\end{gathered}
$$

Vertically asymmetric Ekman friction introduces sign-indefinite terms into the inequality, as well as an additional factor of $\delta$ in front of the baroclinic terms in the two-dimensionality measure. The presence of sign-indefinite terms means that the 
Delta 0.2
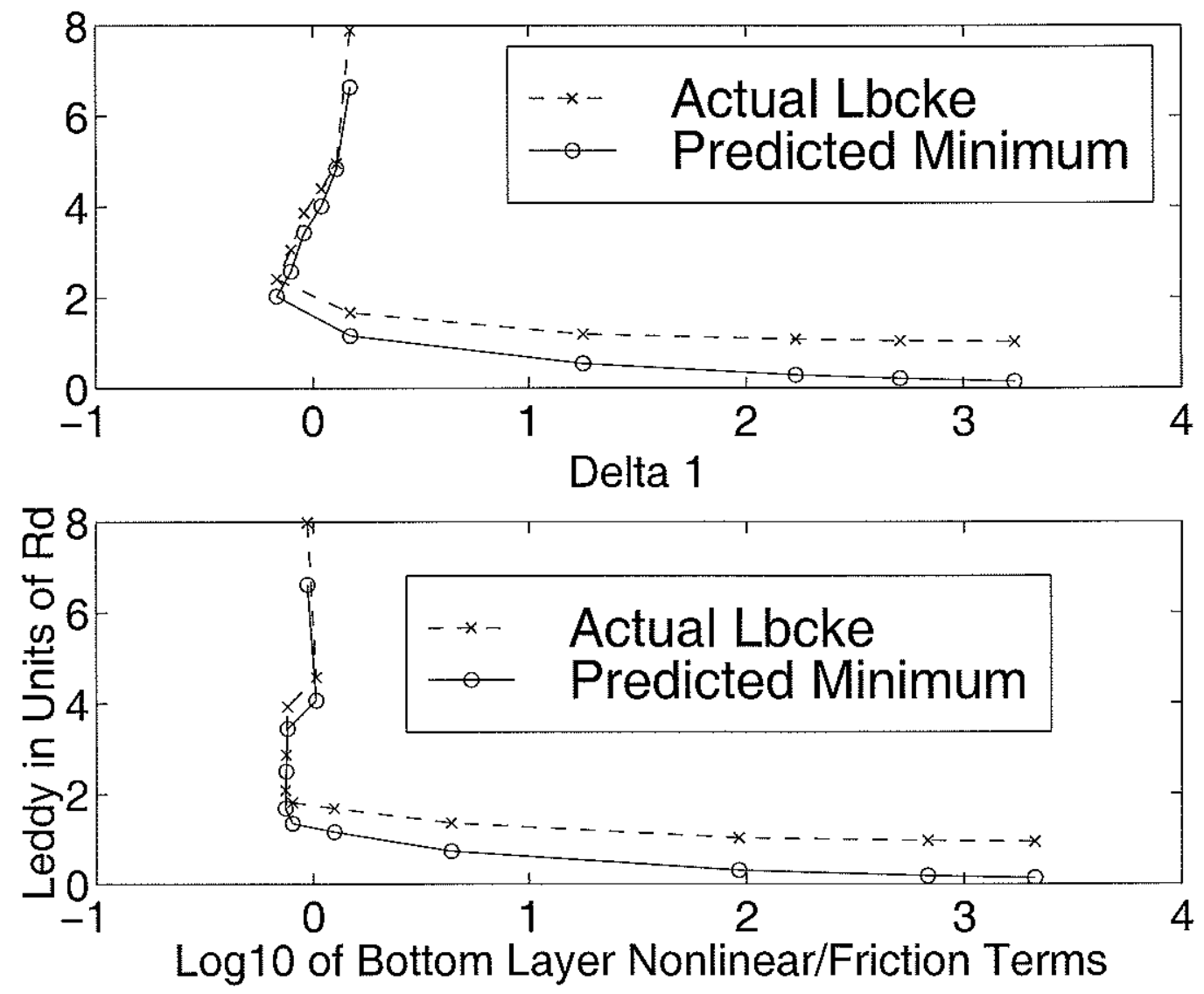

Figure 4-3: "Predicted" (see text) vs actual values of eddy baroclinicity for $\delta=0.2$ (top panel) and $\delta=1$ (bottom panel) symmetric friction experiments. 
precision present with symmetric friction is lost in the bottom friction only case. However, the sign-indefinite terms can be evaluated numerically, and the inequality will still prove useful for interpretation. The top half of figure 4-4 indicates that, in some bottom friction experiments, the two-dimensionality measure exceeds one. In terms of this measure, these experiments have achieved a level of simultaneous baroclinicity and spatial compactness which is impossible under the action of symmetric friction. The bottom half of the figure shows that the sign-indefinite terms take on negative values over most of the domain. They take on their largest negative values in the experiments in which the two-dimensionality measure exceeds one. Thus the sign-indefinite terms could be seen as enabling the eddies to be baroclinic and near $R_{d}$ at the same time.

In bottom friction experiments, the two-dimensionality measure defined in 4.19 exceeds one by a small amount in experiments where sign-indefinite terms take on their largest negative values. But this form of the measure includes a factor of $\frac{H_{1}}{H_{2}}$ in front of the baroclinic terms. In other words, baroclinic terms are downweighted by a factor of 0.2 in the experiments having surface-trapped stratification. The cascade inequality places no bounds on the value of the two-dimensionality measure defined in 4.16, when friction is bottom-trapped. In that form of the measure, the $\frac{H_{1}}{H_{2}}$ factor is not present; equal weight is given to the barotropic and baroclinic enstrophies in assessing the two-dimensionality. We anticipate that this form of the two-dimensionality measure will take on substantially larger values for the $\delta=0.2$, bottom friction experiments, especially since baroclinic kinetic energy is enhanced in these. This is exactly what is seen in figure 4-5. The barrier of one, which could not be exceeded with symmetric friction (irrespective of stratification), and which can be slightly exceeded in bottom friction only experiments with uniform stratification, is far exceeded in experiments with bottom friction and surface-trapped stratification. This indicates that, in terms of the two-dimensionality measure defined in 4.16 , the last set of experiments have achieved the highest levels of simultaneous eddy baro- 


\section{Value of Two-dimensionality Measure, Delta Factor Included}
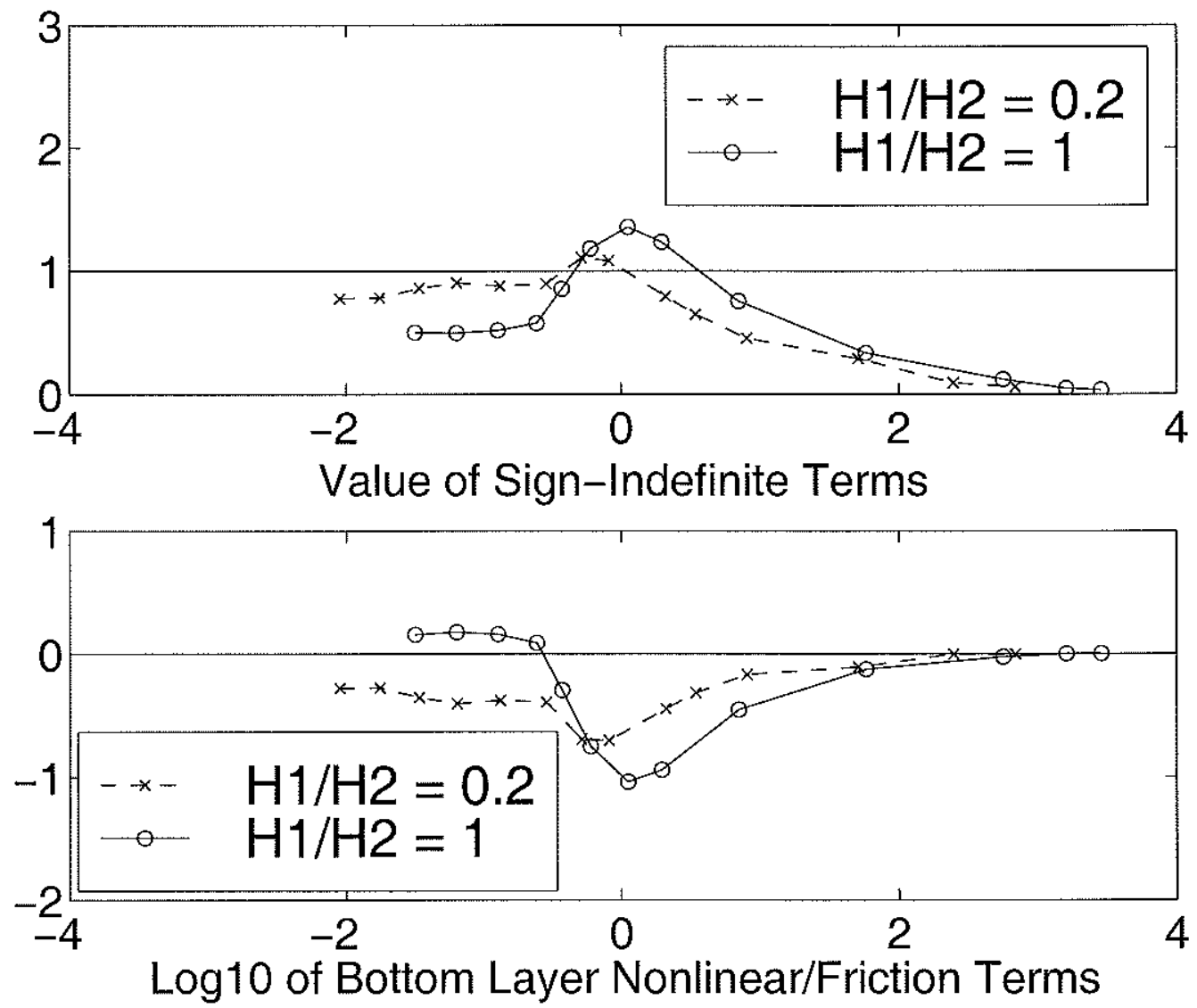

Figure 4-4: Evaluation of $\frac{\left[k_{B T}^{2} K E_{B T}+\frac{H_{1}}{H_{2}} k_{B C}^{2} K E_{B C}\right]}{\left.\mid K E_{B T}\right]}$ and the sign-indefinite terms in inequality 4.19 for the 256 squared bottom friction only experiments, plotted against $\frac{\delta U_{1} L_{2}}{(1+\delta) R_{2} R_{d}^{2}}$. Sign-indefinite terms take on large and negative values, which allows the two-dimensionality measure to exceed one, unlike in the symmetric friction experiments. 
clinicity and compactness. In other words, they are the least "two-dimensional", and most closely resemble the actual mid-ocean eddy field, which is also simultaneously baroclinic and near $R_{d}$.

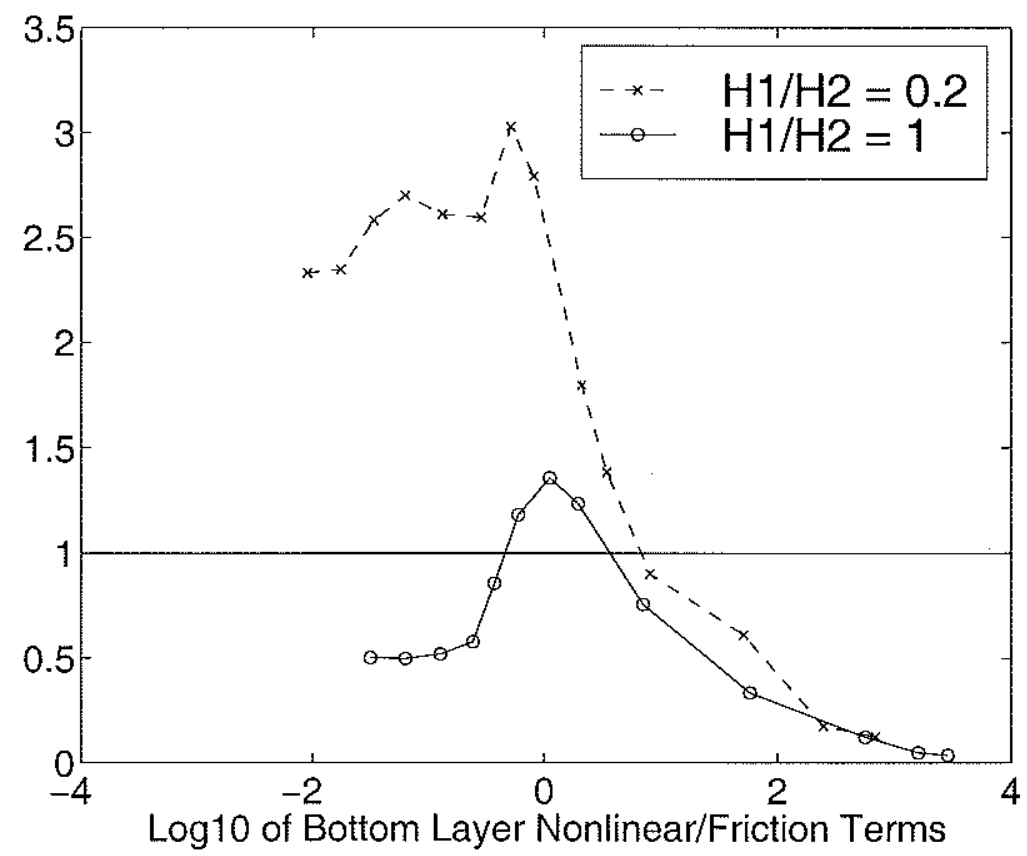

Figure 4-5: Evaluation of $\frac{\left[k_{B T}^{2} K E_{B T}+k_{B C}^{2} K E_{B C}\right]}{\left[K E_{B T}\right]}$ for the 256 squared bottom friction only experiments, plotted against $\frac{\delta U_{1} L_{2}}{(1+\delta) R_{2} R_{d}^{2}}$. Downweighting of baroclinic terms has been removed from the two-dimensionality measure, which then far exceeds one for many of the $\delta=0.2$ experiments.

In experiments with Ekman friction in the bottom layer only, the wavenumber filter must be retained, in order to absorb the enstrophy cascade in the upper layer. We saw in chapter 3 that in bottom friction experiments, the wavenumber filter dissipates most of the potential enstrophy. Is it actually sensible, then, to ignore the contribution of the filter to enstrophy dissipation, as was done in the derivation of the cascade inequality? The fact that enstrophy really is dissipated (and therefore not conserved) in numerical experiments has often been omitted from disussions in the literature on two-dimensional and geostrophic turbulence; see comments at the end of section 3.1. Despite the absence of small-scale dissipation effects in its derivation, 
the inequality we have developed here explains the numerical results rather well. The author has rederived the cascade inequality to account for enstrophy dissipation by the wavenumber filter, which involves turning the enstrophy equation 2.30 into an inequality through use of equation 3.37 before proceeding with the remainder of the derivation. The resulting form of the cascade inequality is, the author believes, too cumbersome to be useful. Thus, the current presentation was deemed best.

The cascade inequality has helped us to interpret our model results. It is tempting to try to apply it to actual ocean data. Direct evaluation of the twodimensionality measure in the real ocean, however, would be difficult, as it would neccessitate separation of modal length scales. Note that the altimetry data used by Stammer 1997 measures sea surface height, which is a combination of modes.

\subsection{Effects of topography on the cascade inequal- ity}

Topography, of both the slope and rough varieties, introduces yet more sign-indefinite terms into the cascade inequality. If these terms become negative, they can reverse the tendency of the inverse cascade to push energy into large-scale, barotropic motions. With topography present we anticipate the two-dimensionality measure to exceed one for a larger range of friction values, or to roll off to small values at a

slower rate. It is still true, however, that the factor $\frac{H_{1}}{H_{2}}$ is introduced when the friction is bottom-trapped, and some topography experiments (not included in this document) indicate that the nature of stratification and friction will therefore still be important in forced-dissipated numerical experiments with topography. 


\subsection{Chapter summary}

In this chapter we develop a cascade inequality for homogeneous geostrophic turbulence forced by an imposed baroclinically unstable mean flow and dissipated by Ekman friction. The inequality is derived from the energy and enstrophy equations, in a manner similar to the arguments made by Fjortoft (1953) for freely evolving one-layer turbulence. We apply the inequality to the four sets of numerical experiments introduced in chapter 3 . The inequality contains a "two-dimensionality measure" which quantifies the amount of energy in the baroclinic mode and in spatial scales near $R_{d}$. The measure is strictly bounded by one in the symmetric friction case, regardless of the value of $\frac{H_{1}}{H_{2}}$, and numerical results strongly support this. Precision is lost when the friction is bottom-trapped because of the presence of sign-indefinite terms. In order to make progress we must resort to numerical evaluation of these. We find that these take on large and negative values, thus enabling the two-dimensionality measure to exceed one in some bottom-friction experiments. Factors of $\frac{H_{1}}{H_{2}}$ appear in the two-dimensionality measure when there is bottom friction. We expect a two-dimensionality measure with this factor omitted to exceed one for many of the $\delta=0.2$ experiments. Thus the cascade inequality points to surface-trapped stratification and bottom-trapped friction as the best combination for producing eddies that achieve high levels of eddy baroclinicity and spatial compactness. The numerical experiments demonstrate this to be true. We therefore argue that the surface-trapped nature of mean ocean stratification, coupled with the bottom-trapped nature of dissipation, may play a fundamental role in allowing eddies to simultaneously retain substantial energy in the baroclinic mode and in horizontal scales comparable to $R_{d}$, as they do in the actual ocean. 


\section{Chapter 5}

\section{Generation of strong eddy fields from nonzonal mean flows on a beta plane}

This part of the thesis focuses on the nonlinear equilibration of geostrophic turbulence forced by nonzonal mean flows. Linear instability analysis indicates that nonzonal flows are inherently more unstable than zonal flows, in the presence of planetary beta. Hence it is possible that the direction of mid-ocean gyre flows may matter a great deal to the amount of eddy energy generated by local baroclinic instability.

We begin with a description of the baroclinic instability process. This will develop intuition useful in interpreting later results. The discussion uses a combination of ideas from Cushman-Roisin (1994) and Glenn Flierl's 12.803 class notes; these draw on ideas originally put forth by Bretherton (1965). Figure 5-1 illustrates the instability mechanism for a zonal mean shear flow on an f-plane. The bottom layer is taken to have no mean flow, while an eastward mean flow is present in the upper layer. By the thermal wind relation, the layer interface slopes upward to the north. A wave, shown in the upper layer, induces north-south displacements of 
vortex tubes. The vortex tube to the left, having moved northward, encounters a decreasing layer thickness $h$. Since $\frac{f}{h}$ is increasing, the tube develops a negative relative vorticity, as shown, in order to conserve potential vorticity. Similar arguments imply a positive relative vorticity in the southward moving vortex tube on the right. In like manner, lower layer vortex tubes moving north (south) develop positive (negative) relative vorticity anomalies due to stretching (squeezing). For reasons of clarity, lower layer waves and vortex tubes are not shown in figure 5-1. Figure 5-2 does show the waves and relative vorticity anomalies in each layer. With an appropriate phase relationship, as indicated, anomalies reinforce each other, thus amplifying wave crests and creating baroclinic instability.

Equations 2.9 and 2.11 indicate that when $\beta=0$, zonal shear flows, no matter how weak, will reverse mean PV gradients between layers. Therefore on an f-plane baroclinic instability exists with arbitrarily small shear. ${ }^{1}$ When mean flows are zonal and planetary beta is larger than the shear-induced PV gradients, gradient sign-reversal no longer occurs between layers. Hence, large planetary beta stabilizes all fluctuations attempting to grow on zonal mean flows. When the mean shear has a meridional component, PV gradients will change sign in the east-west direction. This remains true no matter how large planetary beta becomes-see equations 2.8 and 2.10. Waves varying in the north-south direction, which produce east-west parcel motions, can therefore develop vorticity anomalies from arbitrarily small meridional interface tilts and become unstable. These waves are much like their f-plane counterparts, except that planetary beta inhibits vigorous north-south motions. Although beta does not eliminate instabilities in mean flows which have a meridional component, we will see that it does tighten the range of unstable wavenumbers into an anisotropic band.

Equilibrated eddy amplitudes cannot be obtained from a linear analysis. Sec-

\footnotetext{
${ }^{1}$ Some of the simple facts we present in this discussion are altered in the presence of friction. For now we ignore this effect. Friction is included in the actual stability analyses we perform in this thesis.
} 


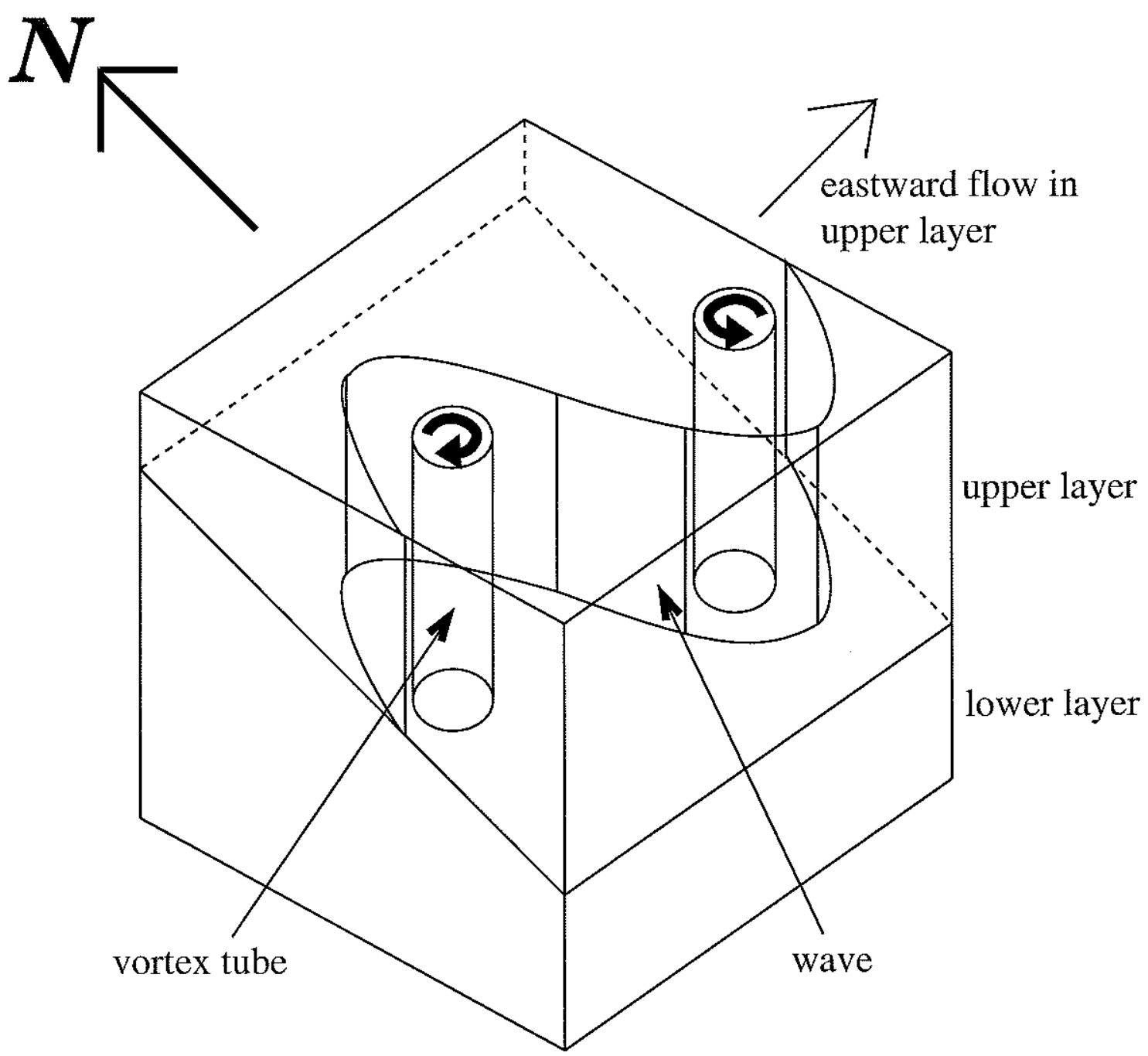

Figure 5-1: First illustration of the baroclinic instability process, adapted from Cushman-Roisin 1994. Here the mean shear flow is purely zonal. 


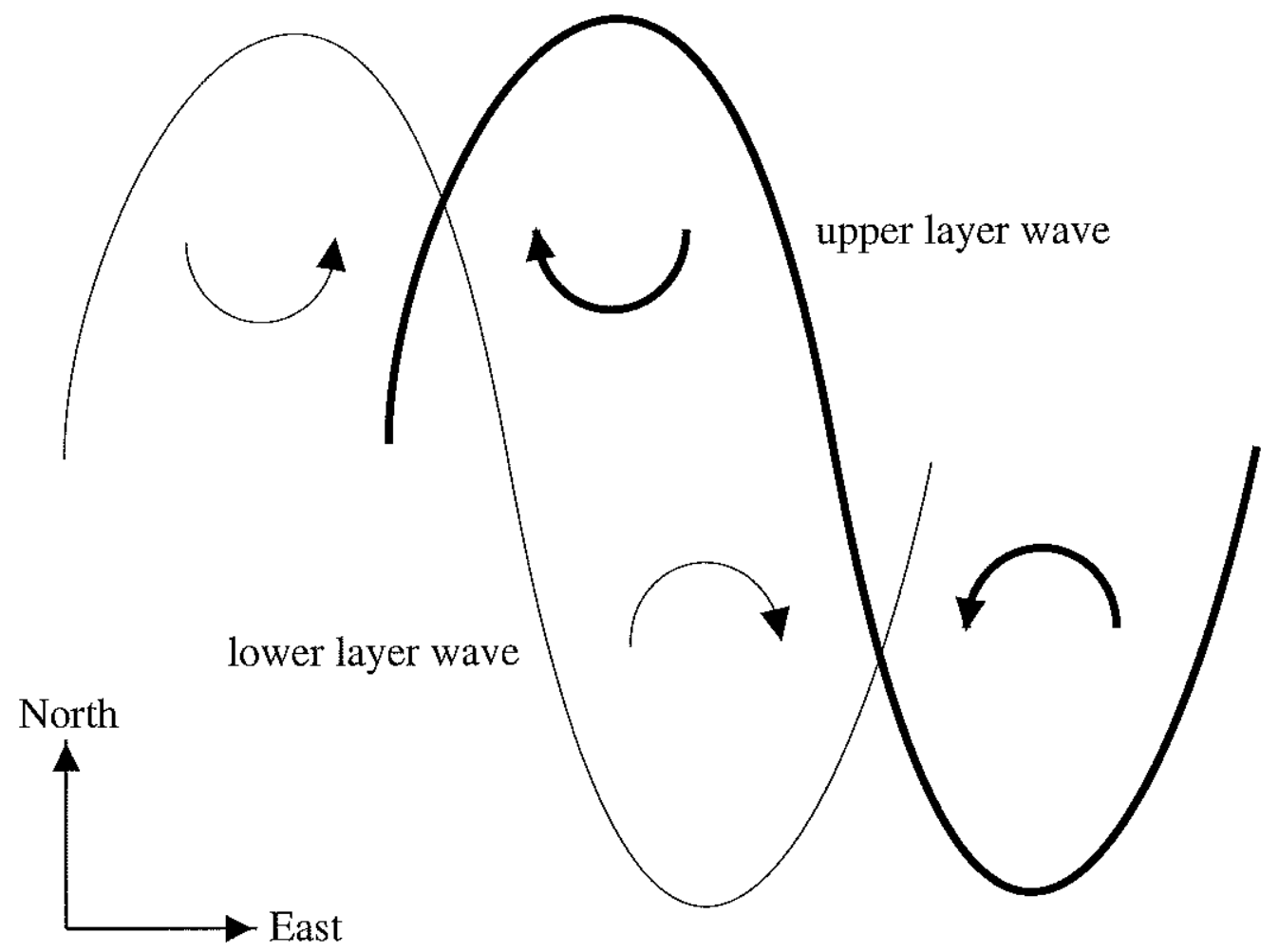

Figure 5-2: Second illustration of the baroclinic instability process, adapted from Glenn Flierl's 12.803 class notes. This is a view looking down on two waves in a zonal two-layer flow. 
tion 5.2 examines fully developed turbulence forced by nonzonal flows. The question we begin with is whether weakly sheared nonzonal flows can generate eddies having velocities much larger than the mean, as observed in the mid-ocean, despite the stabilizing presence of planetary $\beta$. Dependence of energy on angle of shear flow with respect to the east-west direction will be tested. This test will complement the results of Spall (2000), who performed two types of numerical experiments, one on a purely meridional flow, the other on a gyre flow having all angles present. Here, through performance of several experiments with differerent fixed angles, we can ascertain whether eddy energy is generated over the entire gyre, or only in those parts having mean flows that are steeply angled to the east-west direction. The directions of the mean shear vector were chosen to vary from westward to southward; only ninety degrees of the possible 360 was covered. Unless otherwise stated, a $\delta$ value of 0.2 and a bottom-trapped Ekman friction will be used in all of the nonzonal flow experiments in this dissertation.

\subsection{Satisfying $\mathbf{J}(\bar{\psi}, \bar{q})=0$ for homogeneous non- zonal flows}

We intend to perform numerical experiments on a beta plane, with an imposed mean shear flow which is nonzonal. One might ask whether the mean flow will be balanced in such a case, because it is not along lines of constant planetary vorticity. This matters because the governing equations of our model are equations for the fluctuation streamfunction and PV. They could be derived in the following manner, akin to a Reynolds decomposition (c.f. Kundu 1990). We begin with an equation for the full (mean plus fluctuation) streamfunction and potential vorticity:

$$
\frac{\partial q}{\partial t}+J(\psi, q)=F+D
$$


where we have dropped layer subscripts for convenience, $F$ is a forcing, and $D$ is a dissipation. We now divide quantities into a time mean plus fluctuations:

$$
\frac{\partial q}{\partial t}+J(\bar{\psi}, \bar{q})+J(\psi, \bar{q})+J(\bar{\psi}, q)+J(\psi, q)=\bar{F}+F+\bar{D}+D
$$

where quantities without overbars now represent fluctuations. If we assume that:

$$
J(\bar{\psi}, \bar{q})=\bar{F}+\bar{D}
$$

and, in addition, we assume that $\bar{D}+D$ is a linear function of $\bar{q}+q$ (so that $\bar{D}$ is a function only of $\bar{q}$ ) and the forcing is steady (so that $F$ is zero), then 5.3 can be subtracted from 5.2 to give:

$$
\frac{\partial q}{\partial t}+J(\psi, \bar{q})+J(\bar{\psi}, q)+J(\psi, q)=D
$$

This is the fluctuation equation we integrate in our model, where we take $D$ to be an Ekman friction plus a small-scale dissipation. If $\bar{D}+D$ were a nonlinear function of $\bar{q}+q$, or if the forcing were not steady, such a separation would not have been possible. Thus, if one wants to assume that the mean flows arise from forcing and dissipation, one must make special assumptions about the nature of these in order to end up with the fluctuation equations we integrate here. It is simpler instead to assume that the mean flow is balanced:

$$
J(\bar{\psi}, \bar{q})=0
$$

If we now assume that there is no forcing, nor a $\bar{D}$ term, we again arrive at the fluctuation equations.

We show below that, for a chosen shear vector and chosen values of $\delta, \beta$, and $R_{d}$, the equation $J(\bar{\psi}, \bar{q})=0$ can in fact be satisfied for a nonzonal mean flow on a beta 
plane. Satisfaction of $J(\bar{\psi}, \bar{q})=0$ in both layers, along with an imposed magnitude and angle of shear, presents us with four equations:

$$
\begin{aligned}
& \overline{u_{1}} \frac{\partial \overline{q_{1}}}{\partial x}+\overline{v_{1}} \frac{\partial \overline{q_{1}}}{\partial y}=0, \\
& \overline{u_{2}} \frac{\partial \overline{q_{2}}}{\partial x}+\overline{v_{2}} \frac{\partial \overline{q_{2}}}{\partial y}=0, \\
& \overline{u_{1}}-\overline{u_{2}}=z \text { shear }, \\
& \overline{v_{1}}-\overline{v_{2}}=\text { mshear },
\end{aligned}
$$

where zshear and mshear are the zonal and meridional shears, respectively. For most of the nonzonal flow experiments the following will hold:

$$
(\text { zhear, } \text { mshear })=1 \mathrm{~cm} \mathrm{~s}^{-1} \bullet(\cos \theta, \sin \theta),
$$

where $\theta$ is 180 degrees for westward shear, 270 degrees for southward shear. The first two equations can be rewritten using 2.8 through 2.11 :

$$
\begin{aligned}
& \beta \overline{v_{1}}+\frac{\overline{u_{1}} \overline{v_{2}}-\overline{v_{1}} \overline{u_{2}}}{(1+\delta) R_{d}^{2}}=0, \\
& \beta \overline{v_{2}}+\delta \frac{\overline{u_{2}} \overline{v_{1}}-\overline{v_{2}} \overline{u_{1}}}{(1+\delta) R_{d}^{2}}=0 .
\end{aligned}
$$


The four unknowns in equations 5.8 through 5.12 are $\overline{u_{1}}, \overline{u_{2}}, \overline{v_{1}}$, and $\overline{v_{2}}$. The second term on the right hand side of 5.12 is $-\delta$ times the second term on the right hand side of 5.11. Therefore:

$$
\overline{v_{2}}=-\delta \overline{v_{1}},
$$

which, along with 5.9 , determines the meridional velocities. If we now divide 5.11 by $\overline{v_{1}}$, and use 5.13 , we obtain:

$$
\delta \overline{u_{1}}+\overline{u_{2}}=\beta(1+\delta) R_{d}^{2},
$$

which, along with 5.9 , determines the zonal velocities. The solution of the system is:

$$
\begin{gathered}
\overline{v_{1}}=\frac{1}{1+\delta} \text { mshear }, \\
\overline{v_{2}}=-\frac{\delta}{1+\delta} \text { mshear }, \\
\overline{u_{1}}=\beta R_{d}^{2}+\frac{1}{1+\delta} \text { zshear }, \\
\overline{u_{2}}=\beta R_{d}^{2}-\frac{\delta}{1+\delta} \text { zshear } .
\end{gathered}
$$

The mean flow can be balanced in each layer, as long as we are willing to let the layer velocities be adjusted via 5.15 .

The cross product of the layer mean PV gradients is:

$$
\nabla \overline{q_{1}} \times \nabla \overline{q_{2}}=
$$




$$
\begin{gathered}
\left(\frac{\overline{v_{2}}-\overline{v_{1}}}{(1+\delta) R_{d}^{2}}, \beta+\frac{\overline{u_{1}}-\overline{u_{2}}}{(1+\delta) R_{d}^{2}}\right) \times\left(\frac{\delta \overline{v_{1}}-\overline{v_{2}}}{(1+\delta) R_{d}^{2}}, \beta+\frac{\delta \overline{u_{2}}-\overline{u_{1}}}{(1+\delta) R_{d}^{2}}\right)= \\
-\frac{\beta\left(\overline{v_{1}}-\overline{v_{2}}\right)}{R_{d}^{2}} \hat{k},
\end{gathered}
$$

where $\hat{k}$ is the unit vector in the vertical direction. Flows of arbitrary direction on an f-plane, as well as zonal flows on a beta plane, have colinear layer PV gradients. But layer PV gradients are non-colinear for nonzonal flows on a beta plane. Figure 5-3

illustrates geometrically how layer velocities can be chosen to satisfy $J(\bar{\psi}, \bar{q})=0$, for nonzonal mean flows on a beta plane. Once values of $\beta, \delta, R_{d}$, and the shear vector $\vec{S}=\left(\overline{u_{1}}-\overline{u_{2}}, \overline{v_{1}}-\overline{v_{2}}\right)$ are chosen, the layer mean $\mathrm{PV}$ gradients are fixed. Mean flow vectors $\left(\overline{u_{1}}, \overline{v_{1}}\right)$ and $\left(\overline{u_{2}}, \overline{v_{2}}\right)$ must be perpendicular to these gradients in order to satisfy $J(\bar{\psi}, \bar{q})=0$ in both layers. Therefore they will lie along lines ( $L_{1}$ and $L_{2}$ in the drawing) that are not parallel. Unit vectors along $L_{1}$ and $L_{2}$ span the plane. It is therefore possible to find mean flow vectors along $L_{1}$ and $L_{2}$ that satisfy $\vec{S}=\left(\overline{u_{1}}-\overline{u_{2}}, \overline{v_{1}}-\overline{v_{2}}\right)$. With a few stated exceptions, all of the nonzonal flow experiments conducted in the thesis were put through this procedure, to ensure a balanced mean flow. A few comparisons were made between nonzonal experiments with zero lower layer velocity (so that $J(\bar{\psi}, \bar{q})=0$ is not satisfied), and experiments having the same shear and other parameters, but in which the mean flows are balanced. No differences in energy levels or other eddy characteristics were found, indicating that the state of balance in the mean flow does not seem to matter, at least in the homogeneous problem.

\subsection{Eddy generation from weak nonzonal flows on a beta plane: effect of varying angle}

As discussed in chapter 1 , our chosen parameters of $1 \mathrm{~cm} \mathrm{~s}^{-1}$ shear between the layers, a $50 \mathrm{~km}$ deformation radius, and a beta value of $2 \times 10^{-11} \mathrm{~m}^{-1} \mathrm{~s}^{-1}$ do not 


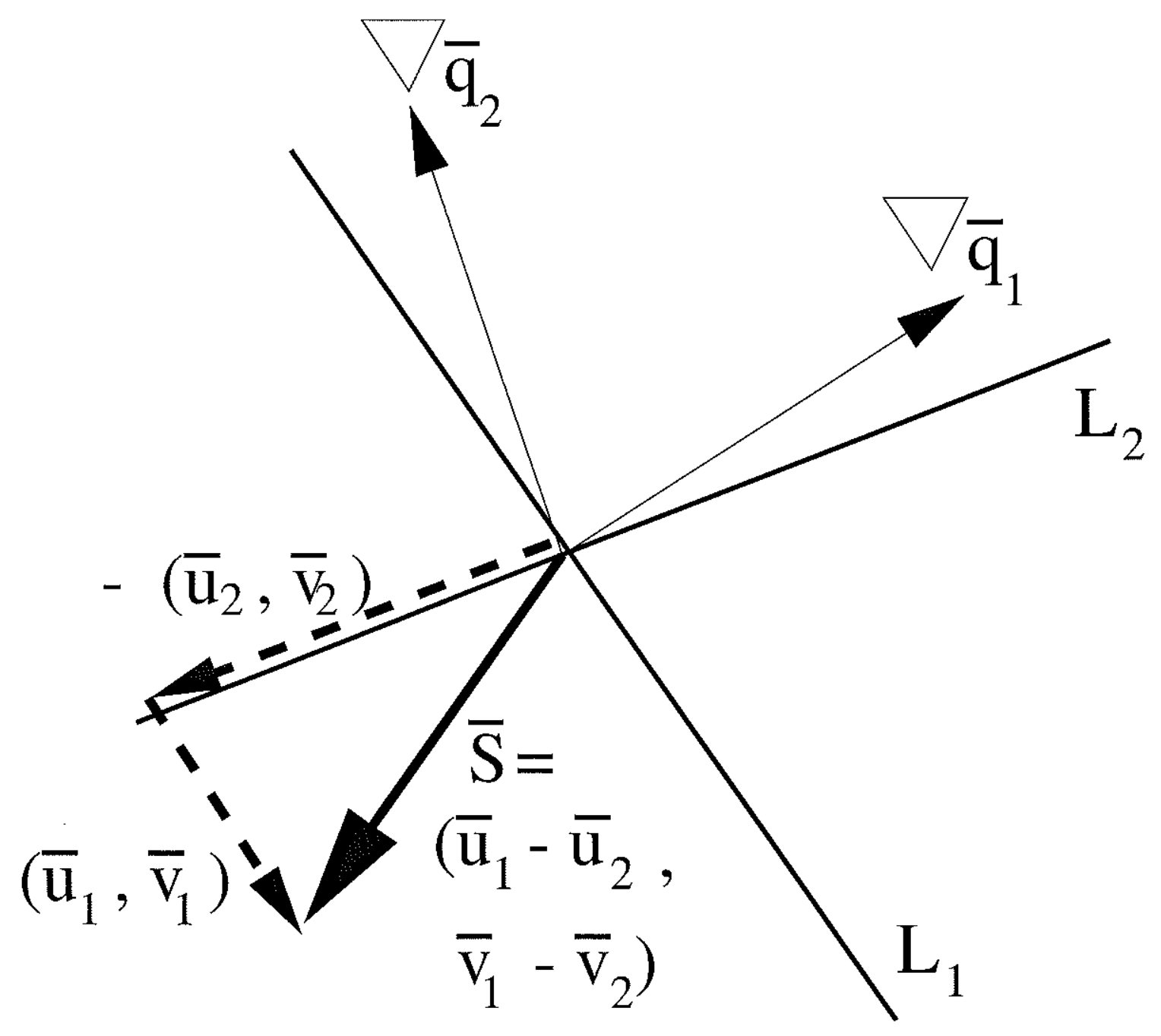

Figure 5-3: Geometrical illustration of the procedure for choosing layer velocities so that $J(\bar{\psi}, \bar{q})=0$ in each layer, for a nonzonal flow on a beta plane. 
satisfy the Charney-Stern criterion for linear instability. Therefore they are linearly stable if zonal. When a 193.4 day bottom friction dissipation time is added ${ }^{2}$, some instabilities exist, with a maximum growth rate of $5 \bullet 10^{-23} \mathrm{~s}^{-1}$. The destabilizating effect of bottom friction has been known for some time (Holopainen, 1961) and will make interpretation of the present results somewhat more difficult. Linear growth rates for nonzonal flows at two different angles are calculated from the stability code and contoured in figure 5-4. Note the different $\mathrm{x}$ and $\mathrm{y}$ scales; there is much more anisotropy present than in the f-plane problem with bottom friction, consistent with earlier discussions. The 185 degree flow has growth rates that are twelve orders of magnitude larger than the zonal case. Maximum growth rates are plotted as a function of the angles we will use in the nonlinear experiments in the top half of figure 5-5. Growth rates continue to increase with increasing angle. Our goal in this section is to determine whether the nonlinear equilibration problem shows a similar increase in eddy energy with angle.

Equilibrated eddy energies (total and upper layer kinetic) are shown as a function of angle in the bottom half of figure 5-5. Experiments were done with shear angles of 270 (southward), 255, 240,225, 210, 195, 190, 185, and 180 (westward) degrees. Thus the experiments have a shear directed south of west. For the westward flow $\overline{u_{1}}=-1 \mathrm{~cm} \mathrm{~s}^{-1}$ and $\overline{u_{2}}=\overline{v_{1}}=\overline{v_{2}}=0$. For the southward flow, $\overline{u_{1}}-\overline{u_{2}}=0$ and $\overline{v_{1}}-\overline{v_{2}}=-1 \mathrm{~cm} \mathrm{~s}^{-1}$. Planetary beta was fixed at $2 \cdot 10^{-11} \mathrm{~m}^{-1} \mathrm{~s}^{-1}$, which fixes the ratio $R_{\beta}=\frac{\text { planetary beta }}{\text { upper layer shear gradient }}=6$. The $270,255,240,225,210$, and 195 degree experiments were spun up from a small scale random initial condition on a 64 squared grid, then interpolated onto a 256 squared grid and continued. Increased resolution did not change the energy levels for any of these save the 195 degree experiment, which increased its energy level dramatically. The energy increase was accompanied by a regime transition, from waves to jets. The 195 degree experiment

\footnotetext{
${ }^{2}$ Nearly all of the nonzonal experiments were done with this value of bottom Ekman friction. Exceptions will be noted.
} 


\section{5 degrees}
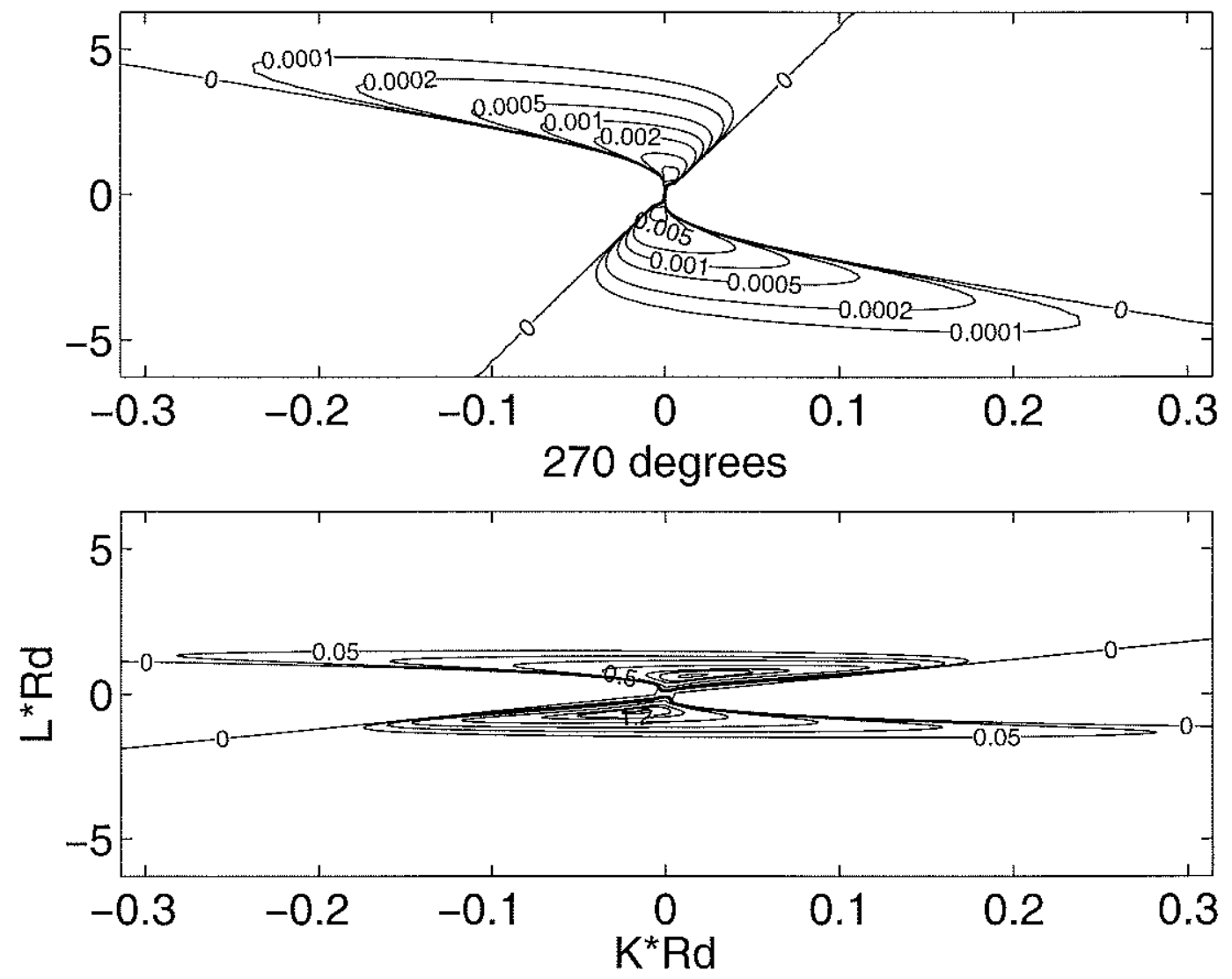

Figure 5-4: Top panel: Linear growth rates in units of $10^{-8} \mathrm{~s}^{-1}$, for a nonzonal 1 $\mathrm{cm} \mathrm{s}^{-1}$ flow angled at 185 degrees (5 degrees south of west), and with a 193.4 day bottom friction dissipation time. Note the anisotropy indicated in the different $\mathrm{K}$ and $\mathrm{L}$ scales. The largest growth rates are for waves having zonal wavenumber $\mathrm{K}$ nearly zero, so that parcel motions are nearly zonal. This is consistent with earlier discussions. Bottom panel: Same but for flow angled at 270 degrees. 

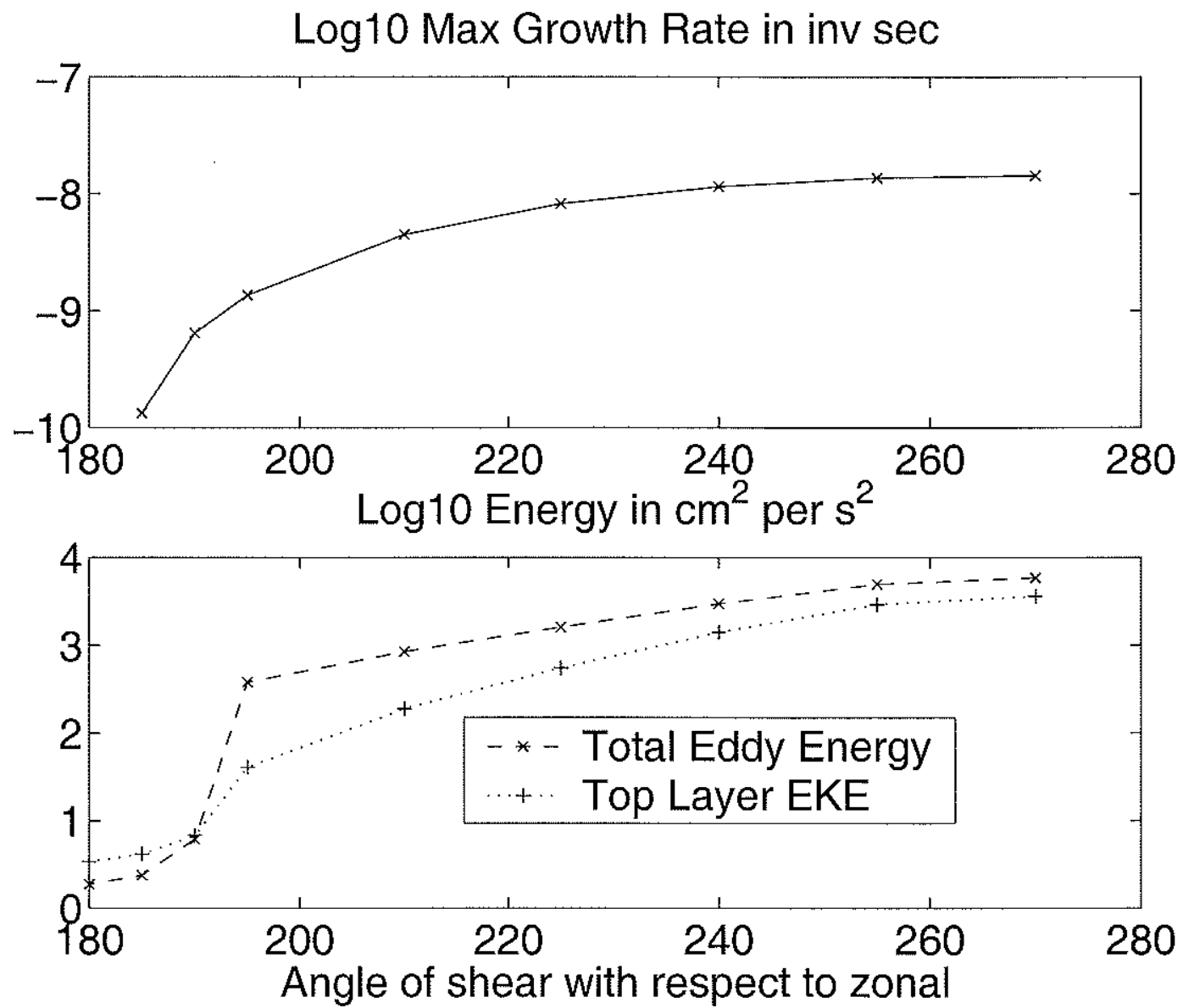

Figure 5-5: Top panel: Maximum linear growth rates in $\mathrm{s}^{-1}$ as a function of angle for southwestward nonzonal flows having shear of magnitude $1 \mathrm{~cm} \mathrm{~s}^{-1}, \beta=2 \bullet 10^{-11}$ $\mathrm{m}^{-1} \mathrm{~s}^{-1}$, and a 193.4 day Ekman friction dissipation time. Bottom panel: Total eddy energy, and upper layer kinetic energy, for the nonlinear equilibration experiments. The magnitudes of shear, planetary beta, and other relevant parameters are held fixed, such that $\frac{\text { planetary beta }}{\text { upper layer shear gradient }}=6$. 
was then run at 512 squared resolution, and energy did not change. The 180, 185, and 190 degree experiments are more difficult to interpret. Those that are plotted were spun up from a small scale random initial condition, and settled into fairly low energy levels. However, when the initial condition was instead taken from the 195 degree experiment, energy levels dropped to only slightly below those in that experiment. This is true even for the zonal flow, which has extremely small linear growth rates. For flow angles that are nearly zonal, final energy levels therefore appear to be strongly dependent on initial condition (and possibly resolution as well). The ability of the zonal flow to retain substantial energy when initialized with a large amplitude fluctuation is reminiscent of the subcritical instability and hysteresis found by Lee and Held (1991). Their model was more complicated than the present one, having radiative damping and boundaries. Ours may be a simpler one in which to study hysteresis. Experiments further investigating the behavior at small angles are ongoing. The essential point, however, remains-where PV gradients induced by the mean flow are much less than planetary beta, eddy energy is a strong function of the angle the shear vector presents to the east-west direction. Total eddy energy increases by an order of magnitude from the 195 degree experiment to the 270 degree experiment, and kinetic energy increases by two orders of magnitude. The 270 degree experiment has a ratio of top layer eddy to mean kinetic energy of about 7200 , which is far too large when compared to observations. This ratio is 81 for the 195 degree experiment, still too large.

Anisotropy is quantified in the top half of figure 5-6. As the mean shear becomes nearly zonal, eddies become ever more zonal. This increasing anisotropy probably arises because, as the mean flow becomes nearly zonal, eddy energy decreases, fluctuation potential vorticities decrease as well. Therefore, parcels are more strongly constrained by planetary beta to flow in a zonal direction. When mean flows are nearly zonal, the ratio of baroclinic to barotropic kinetic energy asymptotes to $\frac{\mathrm{H}_{2}}{\mathrm{H}_{1}}$ $=5$ (bottom half of figure 5-6). As with f-plane experiments having large bottom 
friction, the small angle experiments have very small bottom velocities. Here friction is held fixed, but the forcing effectively decreases as the shear angle approaches 180 degrees and the magnitude of shear is held fixed. This is because eddy motions are nearly zonal. Thus, in the experiments in which the mean shear is nearly zonal, either very small meridional eddy motions extract energy from the large zonal shear, or larger zonal eddy velocities extract energy from a small meridional shearsee equation 2.29. In either case, PV fluxes will be small compared with the case in which mean flows are farther from zonal, in which zonal eddy motions extract energy from large meridional shears. Since energy production is small in the nearly zonal mean shear cases, they resort to minimizing energy dissipation in the bottom as a survival tactic. For mean shear angles far from zonal, eddy energies are large, and so is the degree of barotropization, consistent with the f-plane results of chapter 3.

Snapshots of the upper layer fluctuation streamfunction for these experiments are plotted in figures 5-7 through 5-9. The appearance of zonal bands of eddies is reminiscent of the persistent zonal jets found by Panetta (1993), who also examined two-layer turbulence on a beta plane, with an imposed baroclinically unstable, but purely zonal, shear flow. An example streamfunction from that study (figure 510) shows alternating bands of jets, whereas the present nonzonal experiments are often dominated by one narrow jet. Inhomogeneity of the present experiments is emphasized in figure 5-11, which shows the upper and lower layer fluctuation PV fields, as well as the streamfunctions, for the 195 degree experiment. Zonal averages of the upper layer zonal velocities for the snapshots are plotted in figures 5-12 and 513. The 180 degree experiment is omitted for brevity; it looks very similar to the 185 degree experiment. The zonal average plots, as do the streamfunction plots, indicate a transition from a wave regime (in which energy is spread throughout the domain) at the three smallest angles to a jet regime at 195 degrees (in which energy is concentrated in only one part of the domain). When the mean shear 
Log 10 top layer KEzonal/KEmeridional
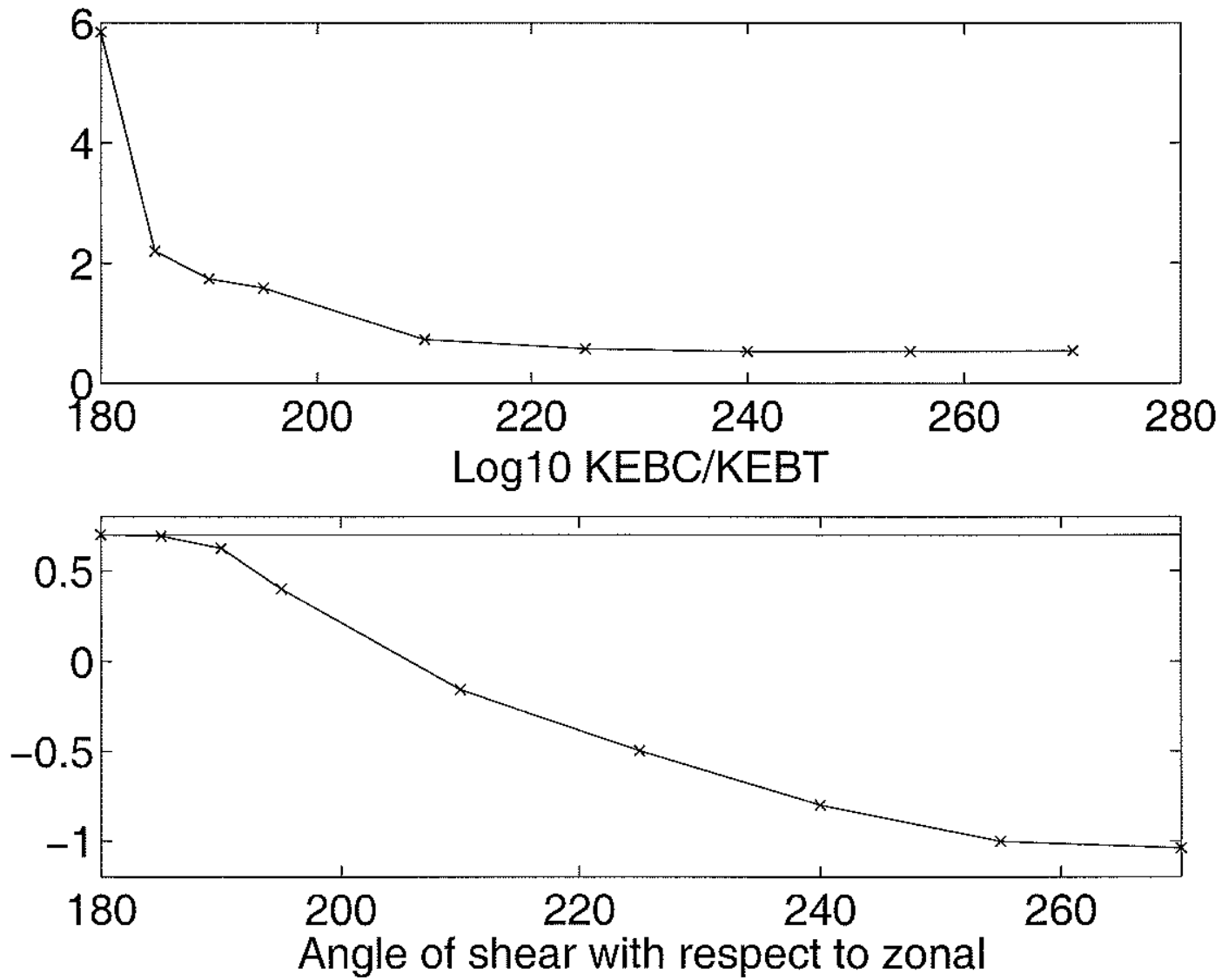

Figure 5-6: Top panel: Anistropy, quantified as the ratio of zonal to meridional kinetic energies in the upper layer, as a function of angle of shear vector with respect to the east-west direction. Bottom panel: Ratio of baroclinic to barotropic kinetic energies, as a function of angle of shear vector. Extra line indicates limiting ratio of $5=\frac{H_{2}}{H_{1}}$; at that ratio the bottom layer velocities are very small. 
flow has a meridional component, zonal motions are able to extract energy from the mean shear. Perhaps that is why, in some of these cases, the eddy field sets itself up to be spatially inhomogeneous, so that there will be divergences of eddy fluxes which can help to equilibrate the jet, which otherwise has little to prevent it from continuing to grow. Figure 5-14 plots the zonally averaged zonal velocity of a snapshot of the 195 degree experiment next to upper layer PV fluxes. Clearly, both zonal and meridional PV fluxes show variations in the north-south direction (note that zonally averaged zonal velocity will be affected by variations in $y$ of the meridional PV fluxes, which are much smaller than the zonal PV fluxes). Thus we have the remarkable result that an eddy field generated by a homogeneous mean flow can set itself up to be inhomogeneous, so that divergences of eddy fluxes will act as an equilibrating mechanism, as they do when acting on the mean flows in weakly nonlinear equilibration studies (c.f. Pedlosky 1970). The plots of zonally averaged zonal velocity indicate that the jets are all of the same sign (eastward flowing). The 195 and 210 degree experiments were negated and then continued, with little difference in energy resulting. Thus the fact that they are all eastward flowing may be a result of the initial condition. Since energy increases between the 195 and 210 degree experiments, which have the same value of planetary beta, it seems unlikely that the controlling width of the front is the $\sqrt{\frac{U_{e d d y}}{\beta}}$ scale emphasized by Panetta (1993) and Rhines (1975). The calculated upper layer Rhines length is $\sim 1.3 R_{d}$ for the snapshot of the 195 degree experiment, $\sim 2.0 R_{d}$ for the snapshot of the 210 degree experiment. Upper layer length scales for these two experiments, calculated as inverses of centroids of the upper layer kinetic energy spectrum, are $\sim 4.8 R_{d}$ and $\sim 1.9 R_{d}$, respectively, for the two experiments. The fronts drift meridionally over time, so that averages taken over long time spans are spatially homogeneous. This ought to be the case, since the forcing is spatially homogeneous. At any instant in time, though, the flow fields do not have to be spatially homogeneous. The degree of inhomogeneity in these experiments is remarkable in comparison to others. Note that 
the domain size in these simulations, $20 \pi R_{d}$, is quite close to that used by Panetta (1993), so differences in the number of jets observed here and in that study are not simply a function of domain width. Experiments with nonzonal mean flows in wider domains are being conducted presently but will not be concluded until after this dissertation is completed. Preliminary indications are that wider domain nonzonal experiments may contain more than one jet. However, the distance between jets still seems to be much larger than the jet width. By that measure even the multi-jet large domain experiments retain an inhomogeneity not present in Panetta (1993)-see the middle upper panel in figure 5-10 for confirmation that the jets in that study are not much narrower than the inter-jet spacing. Panetta has also conducted a study of geostrophic turbulence forced by nonzonal flows but did not send a manuscript in response to repeated requests, so the degree of duplication is unknown. 


\section{0 degrees}

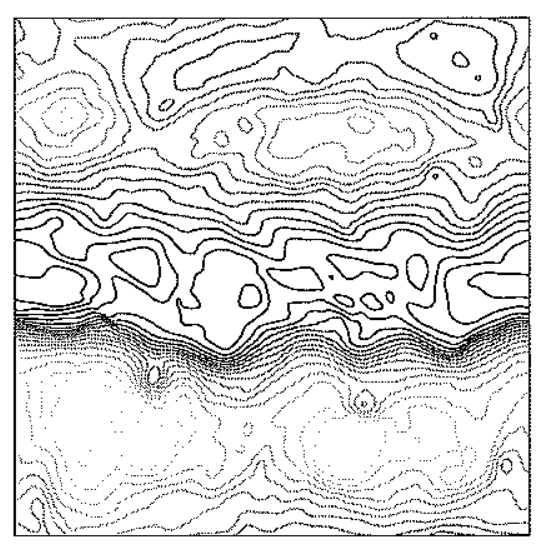

\section{0 degrees}

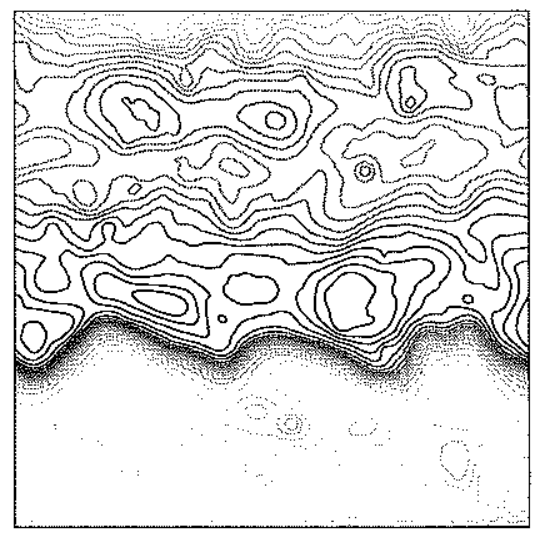

255 degrees

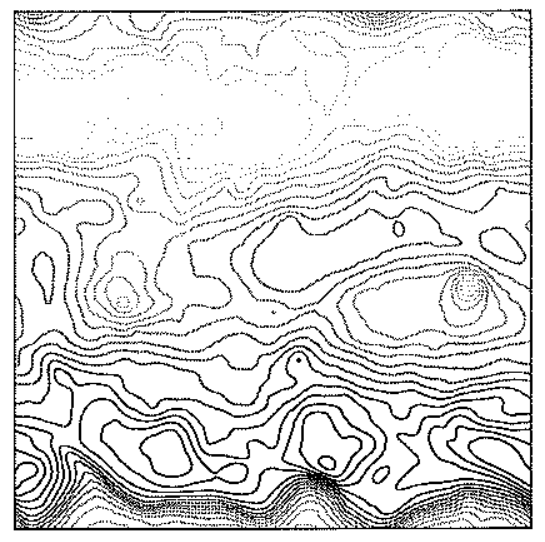

225 degrees

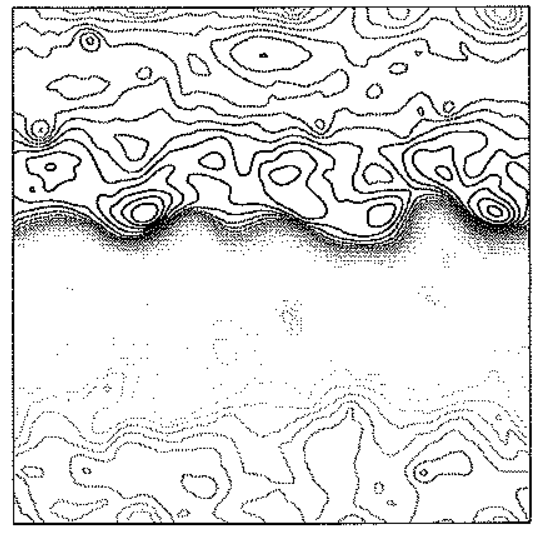

Figure 5-7: Contours of snapshots of upper layer fluctuation streamfunction, as a function of angle of shear vector with respect to the east-west direction. 


\section{0 degrees}

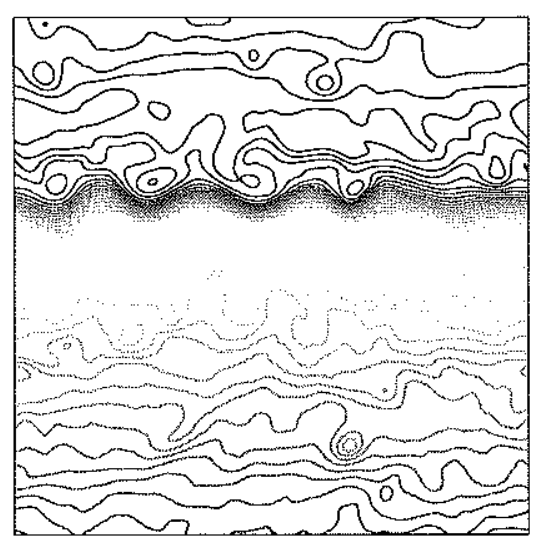

\section{0 degrees}

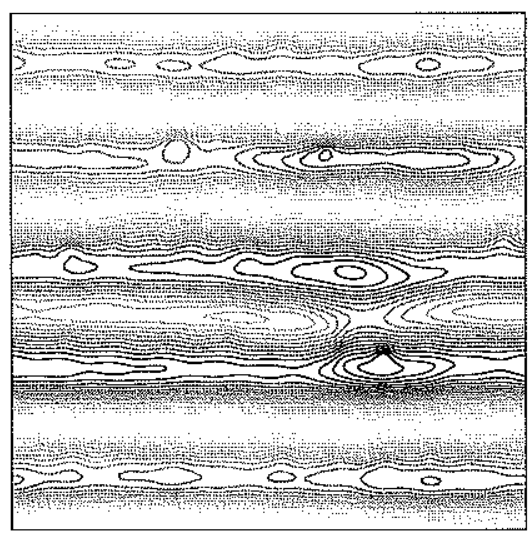

195 degrees

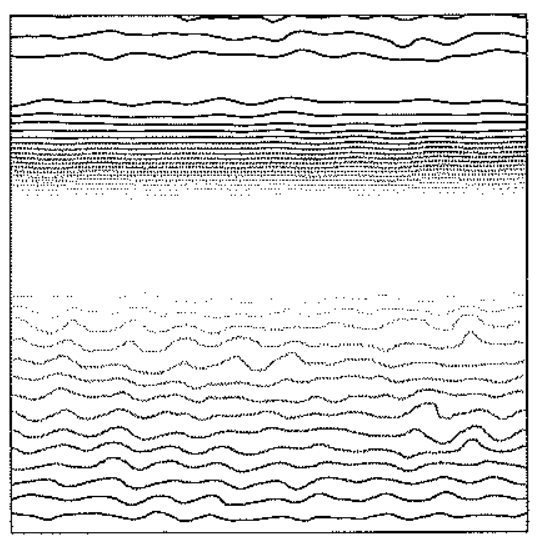

185 degrees

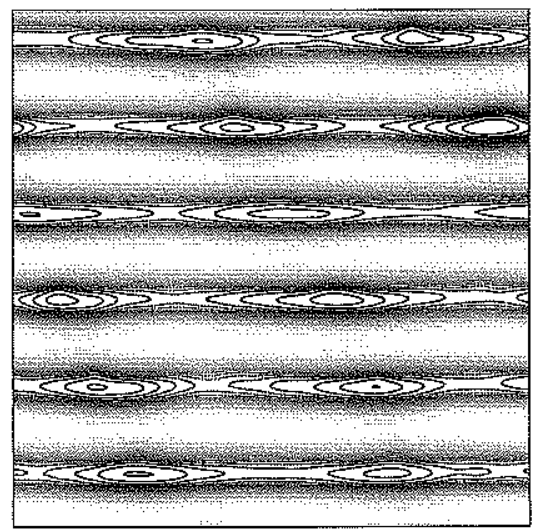

Figure 5-8: Contours of snapshots of upper layer fluctuation streamfunction, as a function of angle of shear vector with respect to the east-west direction. 


\section{0 degrees}

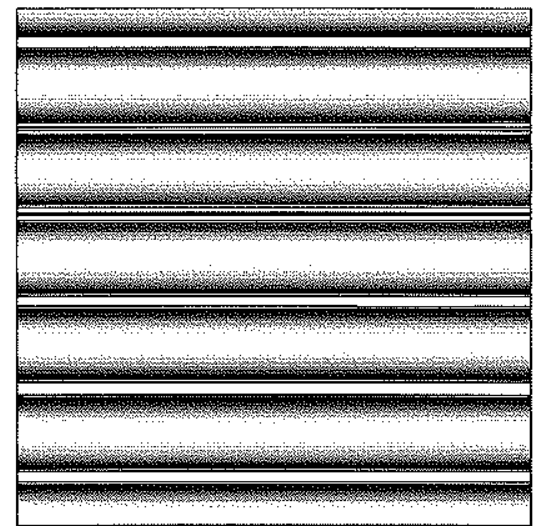

Figure 5-9: Contours of snapshots of upper layer fluctuation streamfunction, as a function of angle of shear vector with respect to the east-west direction. 

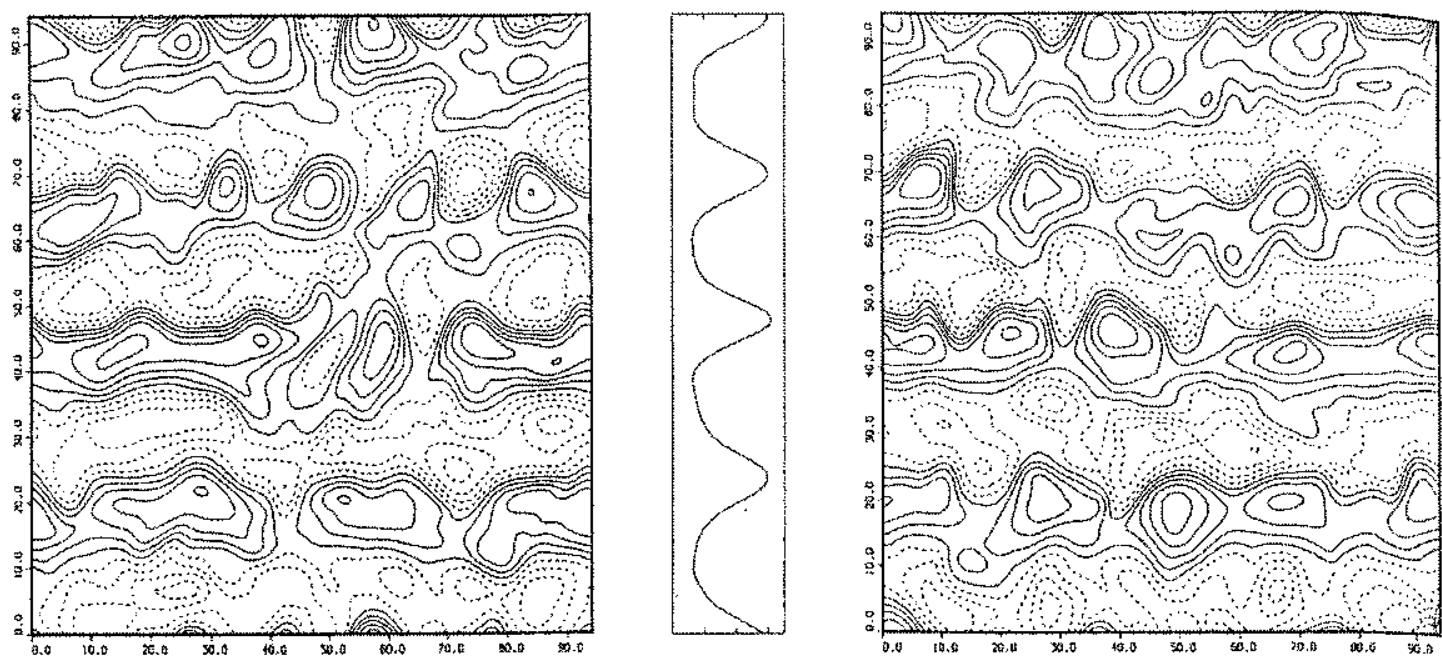

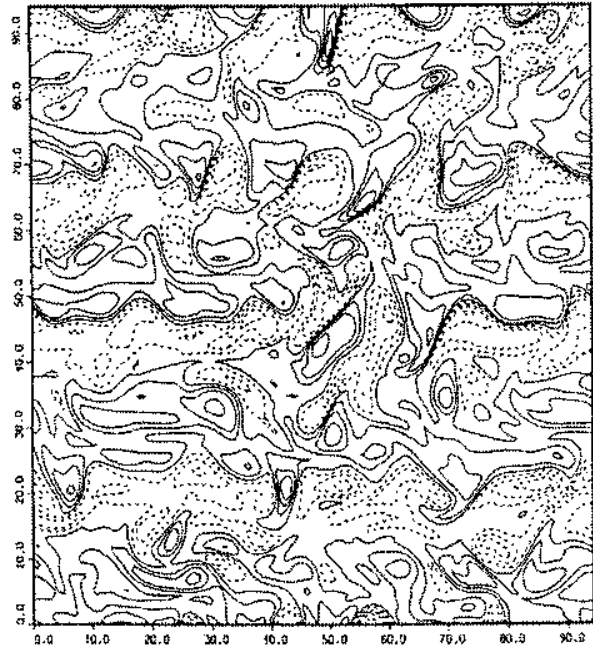

(a)
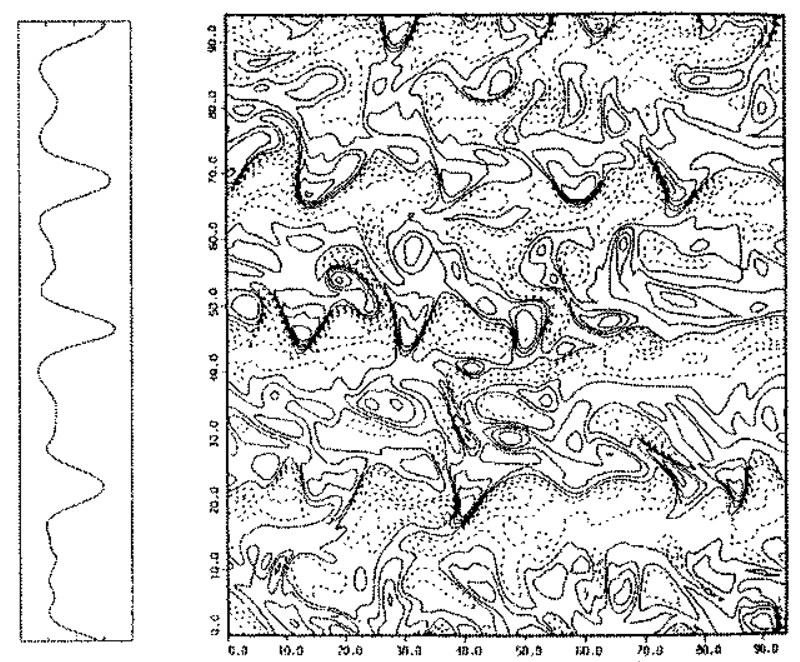

(b)

Figure 5-10: Snapshots of jets generated from an unstable zonal flow on a beta plane in Panetta (1993) (a) Upper layer eddy streamfunction (upper panel) and potential vorticity (lower panel). (b) Same as (a) but for a different time. Between the two panels are time averages of zonally averaged zonal wind and meridional potential vorticity gradient. 


\section{Psi1 195 degrees}

\section{Psi2 195 degrees}
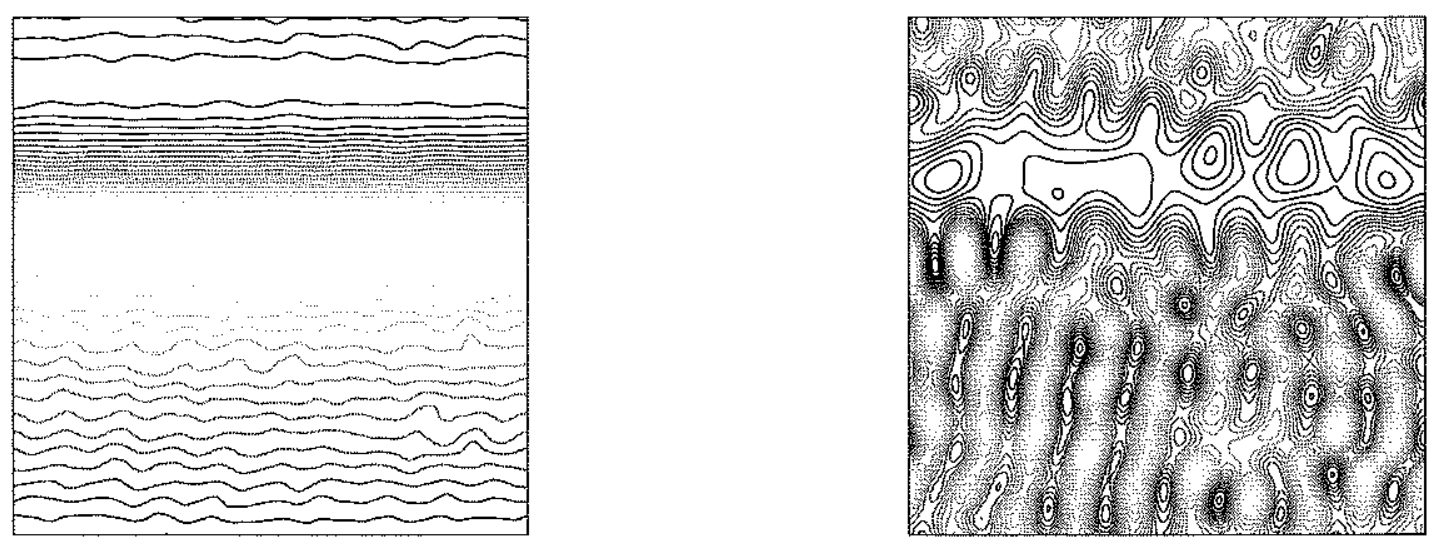

\section{q1 195 degrees}

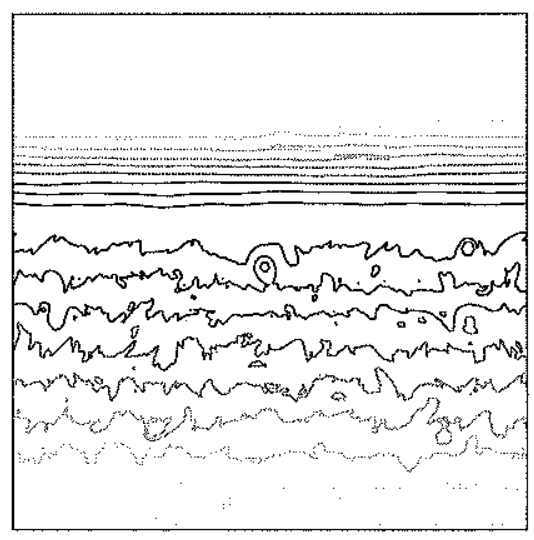

\section{q2 195 degrees}

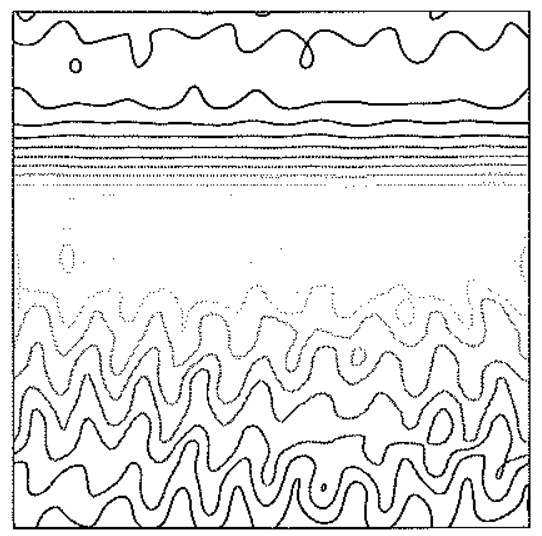

Figure 5-11: Contours of snapshots of upper and lower layer fluctuation streamfunction and PV, for the 195 degree experiment. All four fields are spatially inhomogeneous. 

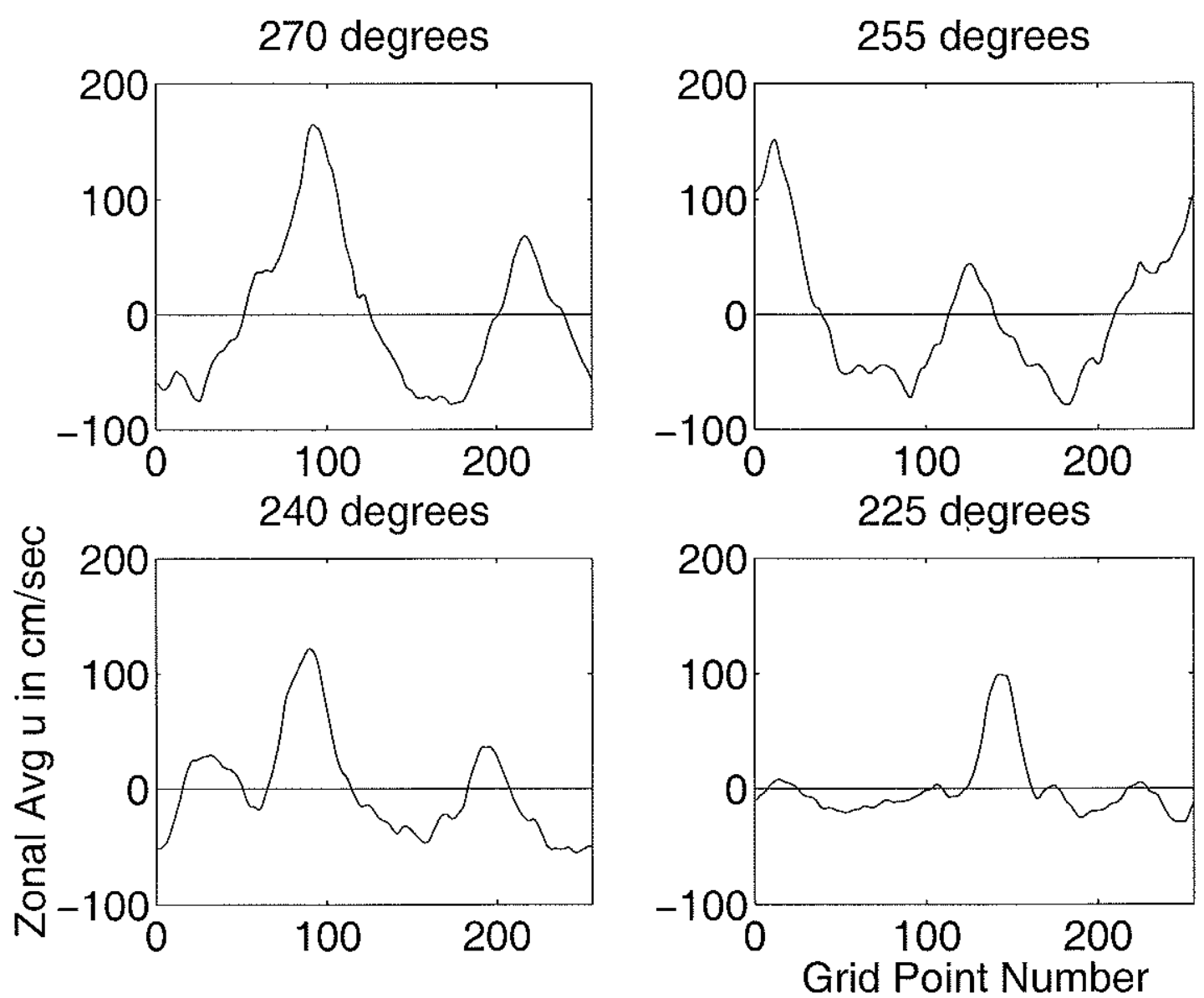

Figure 5-12: Zonally averaged zonal velocity for the streamfunction snapshots shown earlier. 

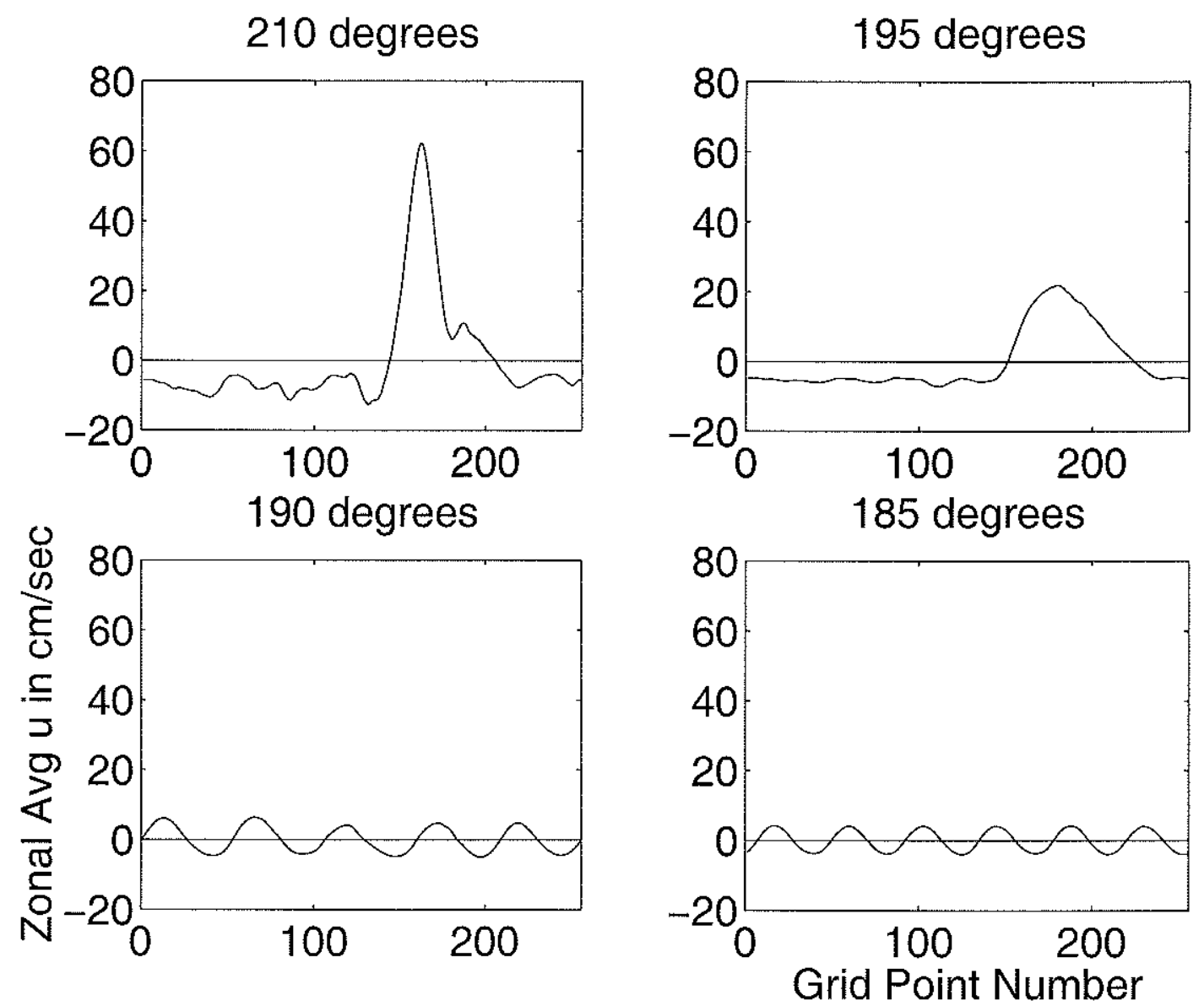

Figure 5-13: Zonally averaged zonal velocity for the streamfunction snapshots shown earlier. 
We have chosen to perform nonzonal flow experiments with a zonal shear component that is directed westward. However, in the regime in which $\frac{\text { planetary beta }}{\text { upper layer shear gradient }}$ is large, somewhat similar results apply when the zonal shear is eastward. A 64 squared experiment with the same magnitude of shear (and other paramters) as the 240 degree experiment, but with the shear directed 60 degrees south of east, has about 46 percent less energy, a higher ratio of $\frac{K E_{B C}}{K E_{B T}}(0.28$ compared to 0.16$)$, and a slightly higher upper layer anisotropy ratio $\frac{K E_{z o n a l}}{K E_{\text {meridional }}}$ (3.40 compared to 3.13 ). Figure 5-15 indicates that a single jet is present in this experiment as well. The different energy levels indicate an asymmetry between eastward and westward flows. This will be discussed in more detail in chapter 6 , where a more dramatic asymmetry will be found.

We have shown that the energy of an eddy field forced by a weak nonzonal mean flow on a beta plane $\left(\frac{\text { planetary beta }}{\text { upper layer shear gradient }} \gg 1\right)$ is a strong function of angle, and that meridional flows can produce large ratios of eddy to mean kinetic energy (in fact, generally too large, when compared to observations). This complements the work of Spall (2000), which showed that nonzonal flows present in the interior of a wind-driven gyre flow can produce eddy kinetic energies larger than that of the mean. In a gyre flow, a number of angles are present at once. Here we isolate the effects of angle by performing several experiments, each with a different fixed angle. Taken at face value, the present experiments indicate that in a gyre, it is the regions having flows that are nearly meridional that produce the bulk of the eddy energy. The physical appearance of the eddy fields here, however, is quite different from those in Spall (2000). The absence of boundaries allows zonal motions such as jets to flourish. Spall's study, conducted in a bounded domain, found no such marked anisotropy in the eddy fields. As a general statement, eddy kinetic energy in the mid-ocean is nearly isotropic (c.f. Table 1 in Wunsch 1997). It is possible, of course, to produce isotropic eddy fields in a doubly periodic domain. We saw little anisotropy in the f-plane experiments in earlier chapters. We anticipate that 


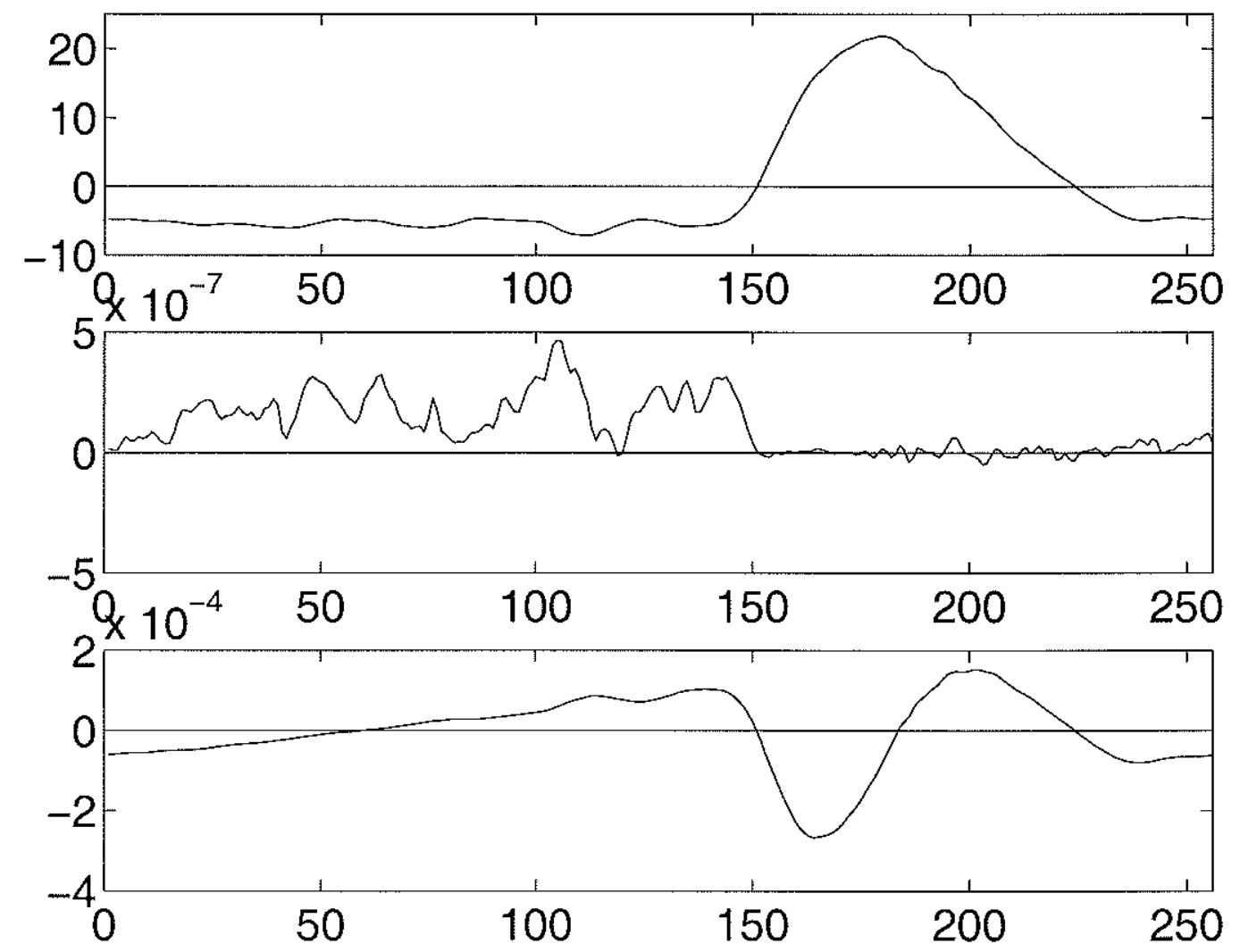

Figure 5-14: Top panel: Zonally averaged zonal velocity for snapshot of 195 degree experiment. Units $\mathrm{cm} \mathrm{s}^{-1}$. Middle panel: Zonally averaged meridional PV flux $v q$. Bottom panel: Zonally averaged zonal PV flux $u q$. Units for the PV fluxes are cm $\mathrm{s}^{-2}$. The $\mathrm{x}$-axis is gridpoint number, running from south (1) to north (256). 


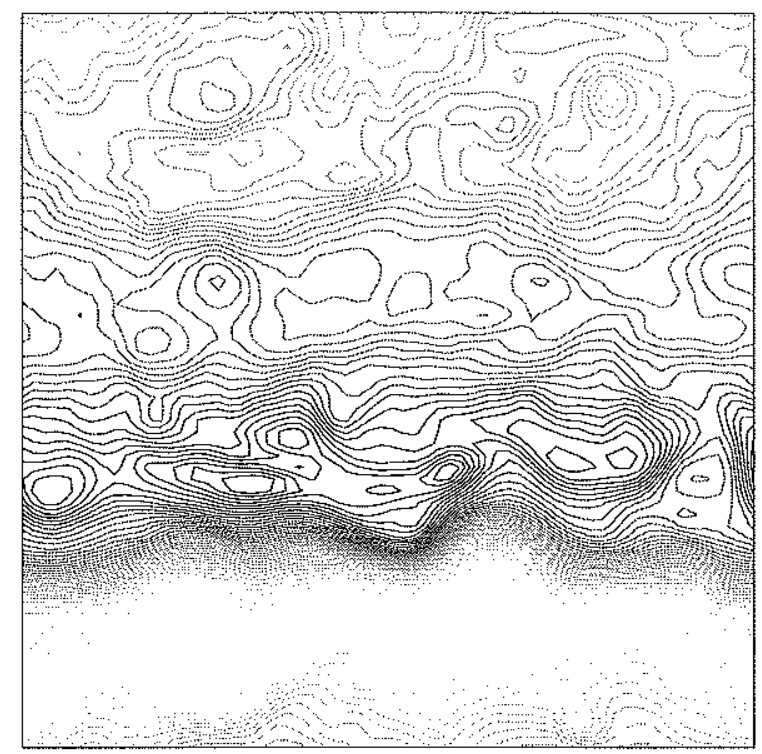

Figure 5-15: Contours of upper and layer fluctuation streamfunction for an experiment with shear directed 60 degrees south of east.

anisotropy will decrease in nonzonal flow experiments if we reduce planetary beta, while keeping the magnitude of shear fixed. Then the PV gradient induced by shear will be comparable in size to planetary beta. We have advertised the ocean interior as an environment in which shear-induced PV gradients are much less than planetary beta. However, maps produced by Keffer (1985) show that this may not be the case (figures 5-16 and 5-17). In subtropical gyres it is not uncommon for potential vorticity gradients in the upper thermocline to be nearly perpendicular to those in the lower thermocline (which are directed north-south, as one would expect when planetary beta dominates-note that abyssal PV maps produced by O'Dwyer and Williams 1997 show some departures from zonality, but not so much in the Pacific, thus, PV gradients are likely to change direction with depth over much of the world ocean). It is possible, therefore, that we have underestimated the size of the shear gradients. This may be a natural consequence of the two layer model used in this research. In a two layer model, one layer is taken to represent the thermocline. But the Keffer maps indicate much vertical variation within the thermocline, which 
cannot be represented in a two layer model. The ocean interior may actually be better represented by experiments in which shear-induced and planetary gradients are comparable, which we achieve in the next section by reducing beta. Equilibrated eddy fields will be examined as a function of the ratio $R_{\beta}=\frac{\text { planetary beta }}{\text { upper layer shear gradient }}$ for a fixed magnitude and angle of shear flow (other parameters are fixed as well). Our expectation is that the degree of anisotropy will reduce as this ratio decreases. We will find this to be true. As in the current section, we will also uncover some unexpected results. 


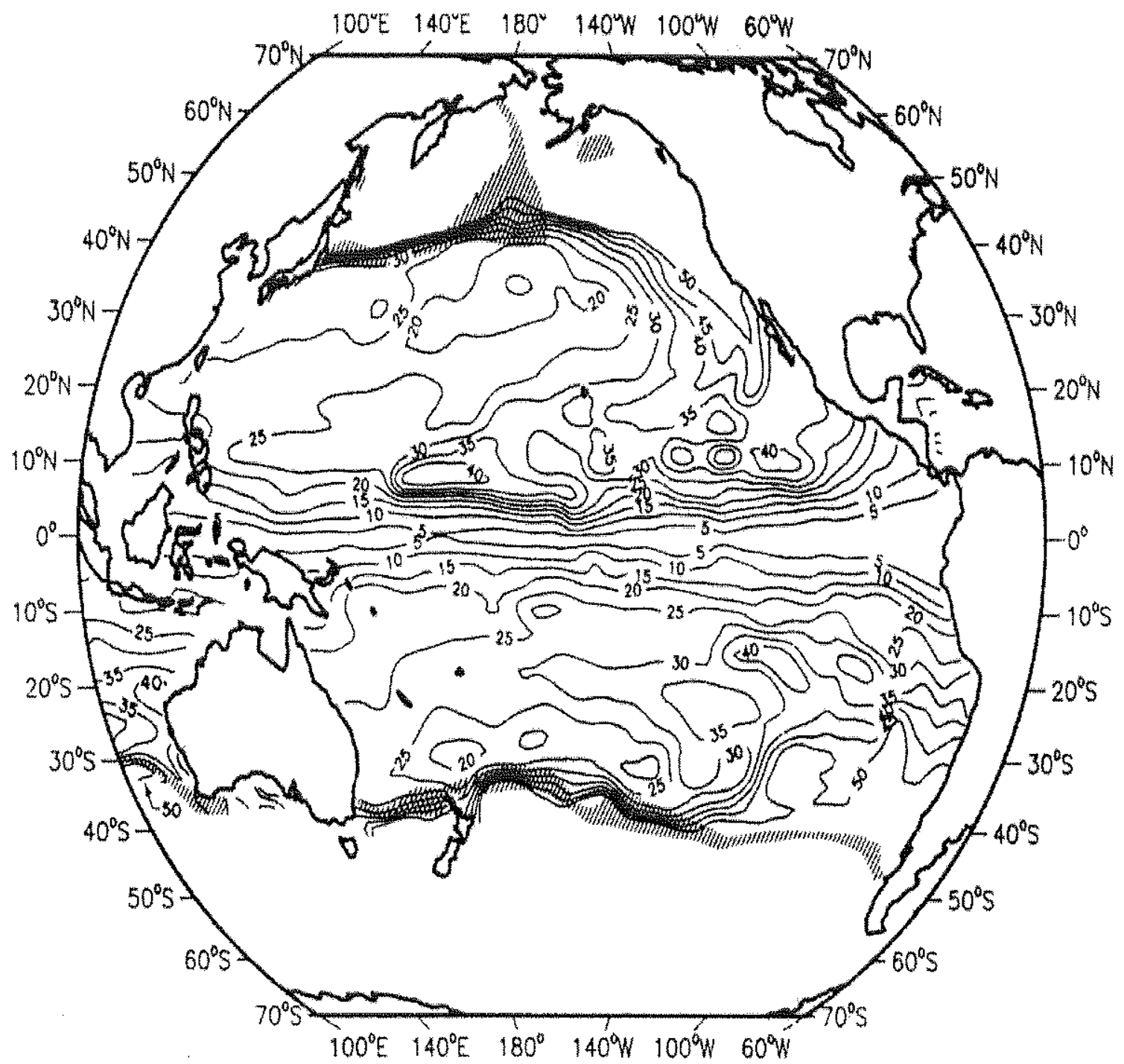

Figure 5-16: Potential vorticity $\frac{f}{\rho} \frac{\partial \rho}{\partial z}$, where $f$ is the Coriolis parameter, $\rho$ is density, and $z$ is the vertical coordinate, of the $\sigma_{\theta}=26.05-26.25$ layer in the Pacific upper thermocline (the middle of this layer is at a depth of about 200 to 400 meters over much of the Pacific subtropical gyre). Units are $10^{-13} \mathrm{~cm}^{-1} \mathrm{~s}^{-1}$. From Keffer (1985). 


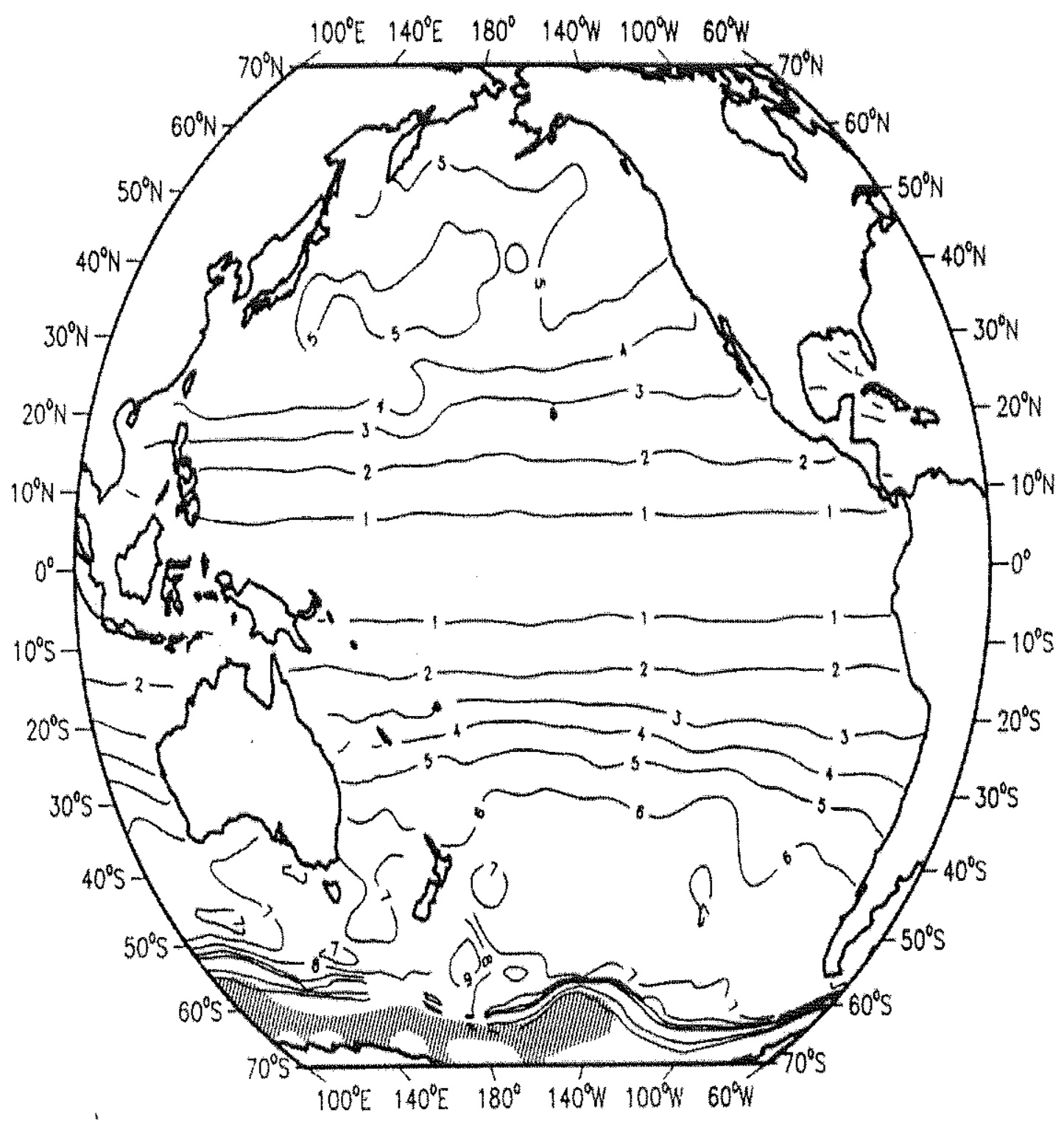

Figure 5-17: Potential vorticity of the $\sigma_{\theta}=27.3-27.5$ layer in the Pacific lower thermocline (the middle of this layer is at a depth of about 1000 meters over much of the Pacific). Definition of PV and units same as in previous plot. From Keffer (1985). 


\subsection{Effect of varying beta on isotropy of eddies generated from nonzonal mean flows}

In the next set of experiments, the angle of the shear vector was fixed at 210 degrees (30 degrees south of west), while planetary beta was varied. The anisotropy of this set of experiments is plotted in figure $5-18$. The large $R_{\beta}$ regime is dominated by zonal motions, while the small $R_{\beta}$ regime (and the f-plane result with the same Ekman friction) is isotropic. These experiments were performed with both hyperviscosity and wavenumber filter as small-scale dissipation schemes. The reasons for this will be discussed shortly.

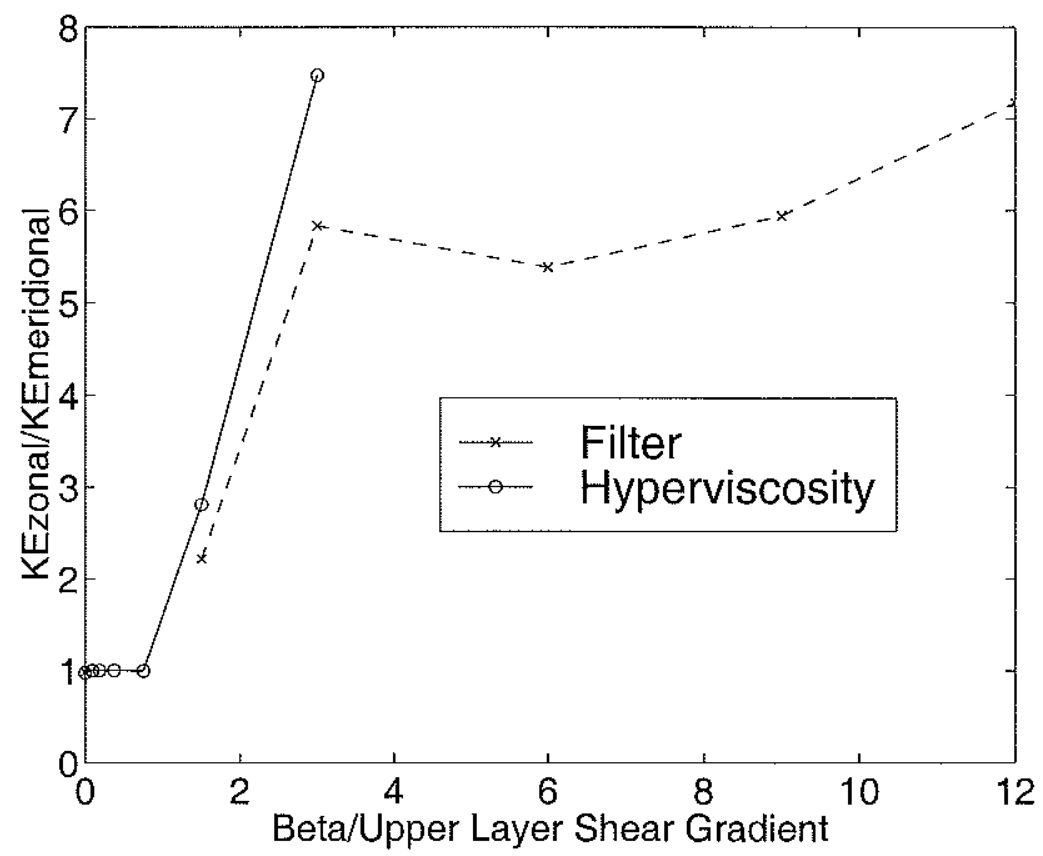

Figure 5-18: Anisotropy of experiments with fixed magnitude and angle of shear, but varying planetary beta. Points marked with an " $\mathrm{x}$ " were integrated in a 256 squared domain with the wavenumber filter; " 0 " denotes experiments in a 128 squared domain with hyperviscosity.

The angle between layer PV gradients is plotted as a function of $R_{\beta}$ in figure 519. For $R_{\beta} \gg 1$ the angle is quite small, while for $R_{\beta}=0$ the angle is 180 degrees. Anisotropy is plotted as a function of the angle between layer gradients in figure 5- 
20. The transition between anisotropic and isotropic regimes occurs when the angle is approximately 90 degrees; more experiments are neccessary to pin down the transition more accurately. When there is a small angle between gradients, there is effectively an imposed direction, and anisotropy develops in the eddy field. Larger angles between gradients implies that there is no imposed direction, and the eddy fields are isotropic.

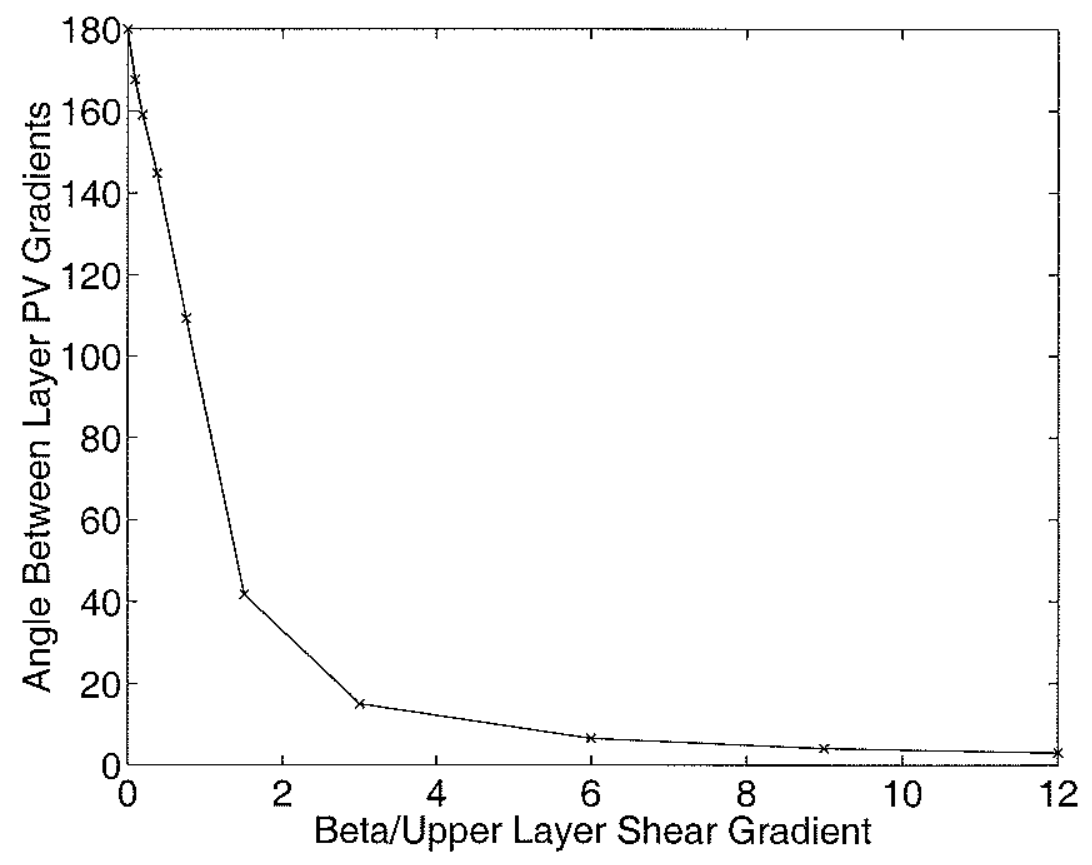

Figure 5-19: Angle between layer PV gradients as a function of $\frac{\text { planetary beta }}{\text { upper layer shear gradient }}$.

Figures 5-21 and 5-22 plot total eddy energy and eddy kinetic energy versus $R_{\beta}$. Planetary beta is often thought of as a stabilizing influence on eddies. However, our experiments with large beta contain more energy than the f-plane result. Also, for the large $R_{\beta}$ experiments, energy increases as beta increases. This means that in the present experiments, eddy kinetic energies cannot be explained by the scaling of Spall (2000), which is: 


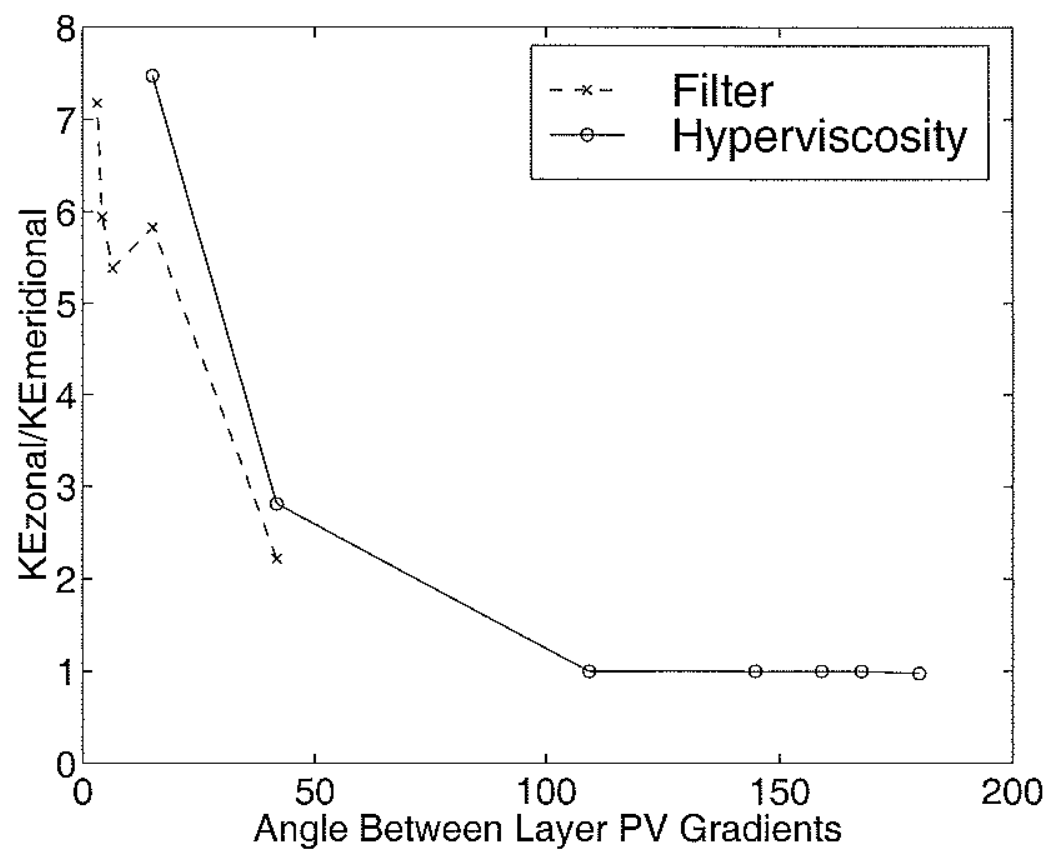

Figure 5-20: Anisotropy as a function of angle between layer PV gradients.

$$
\left(\frac{U_{\text {eddy }}}{U_{\text {meanflow }}}\right)^{2}=\left[\frac{U_{\text {meanflow }}}{\beta R_{d}^{2}}\left(\frac{L_{\text {basin }}}{R_{d}}\right)^{2}\right]^{\frac{2}{3}},
$$

meaning that energy should decrease with increasing beta. Differences in the nature of eddy fields between the current experiments (which are jet dominated) and the Spall (2000) gyre calculations (no jets) are probably behind the fact that the gyre scaling does not work here. Another difference is that there is no $L_{b a s i n}$ in a doublyperiodic domain. The most unexpected result of the plot is the emergence of a strongly energetic regime that arises when $R_{\beta}$ is greater than zero but less than one (or so; again, more experiments are required to firmly establish the criterion). This regime was discovered in 64 squared runs. When resolution increased to 128 squared and 256 squared, the qualitative appearance of eddy fields remained the same, but energies did not equilibrate-they continued to rise-after a few months of numerical integration. Strong resolution dependence was observed in only a few other experiments in this thesis, the small angle experiments of the last section. In 
order to obtain results in a feasible time, we performed experiments in the weak beta regime using hyperviscosity as our small-scale dissipation. The qualitative nature of eddy fields under hyperviscosity is the same as with the filter, but equilibration takes place in about one week of processor time. Results should be independent of resolution with a fixed coefficient and power of hyperviscosity. This was checked and found to be true. More details of the hyperviscosity parameters will be discussed in the next chapter. Note that three values of $R_{\beta}$ were run with both hyperviscosity and wavenumber filter, with little difference in energy resulting.

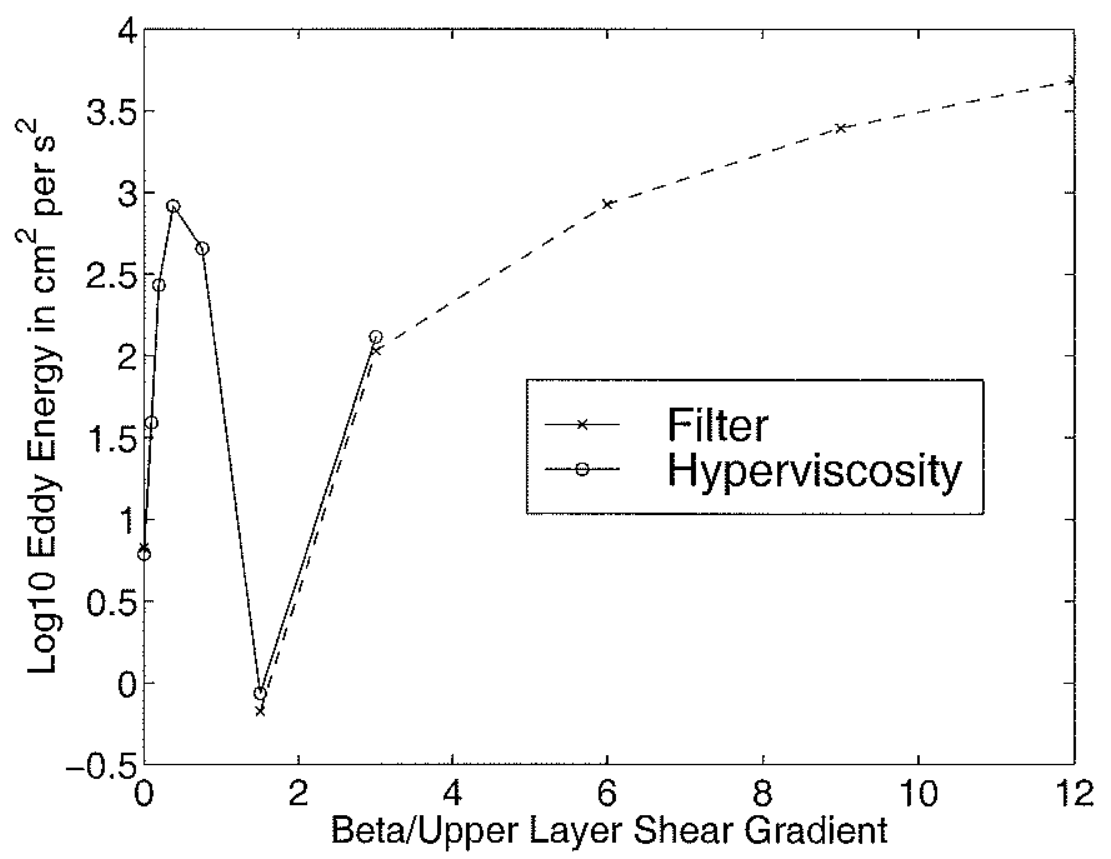

Figure 5-21: Total eddy energy as a function of $R_{\beta}=\frac{\text { planetary beta }}{\text { upper layer shear gradient }}$ for a nonzonal flow of fixed shear and angle (30 degrees south of west).

Eddy baroclinicities are plotted in figure 5-23. For large values of $R_{\beta}$, energy increases as $R_{\beta}$ increases. Along with increased energy comes increased barotropization, consistent with the results of chapter 3 . None of these experiments is close to the limit $\frac{K E_{B C}}{K E_{B T}}=5$, meaning that lower layer velocities are substantial.

Snapshots of the upper layer fluctuation streamfunction for these experiments are plotted in figures 5-24 through 5-27. The experiments with $R_{\beta}$ values greater 


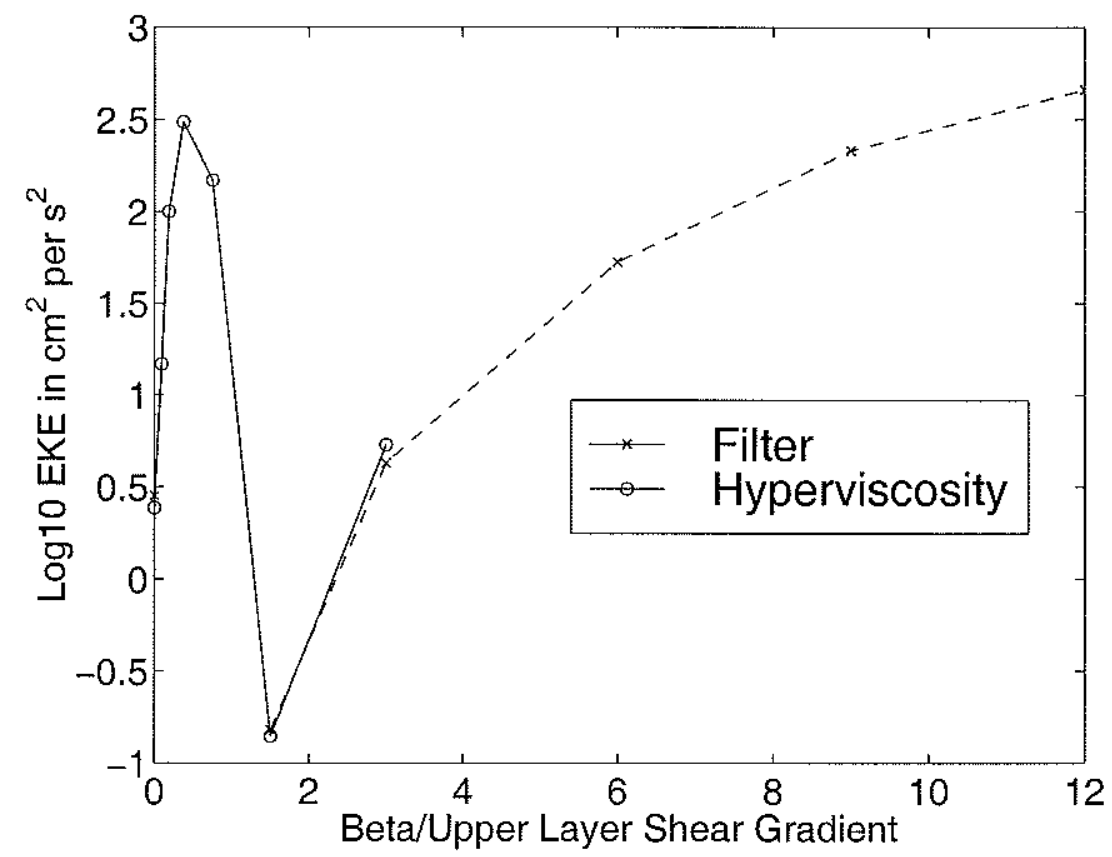

Figure 5-22: Total eddy kinetic energy as a function of $R_{\beta}$ for a nonzonal flow of fixed shear and angle.

than or equal to 3 are zonal in appearance and contain one narrow front. The two experiments having $\frac{\text { planetary beta }}{\text { upper layer shear gradient }}=1.5$, but with different small-scale dissipations, both hint at transition between anisotropic and isotropic regimes. 


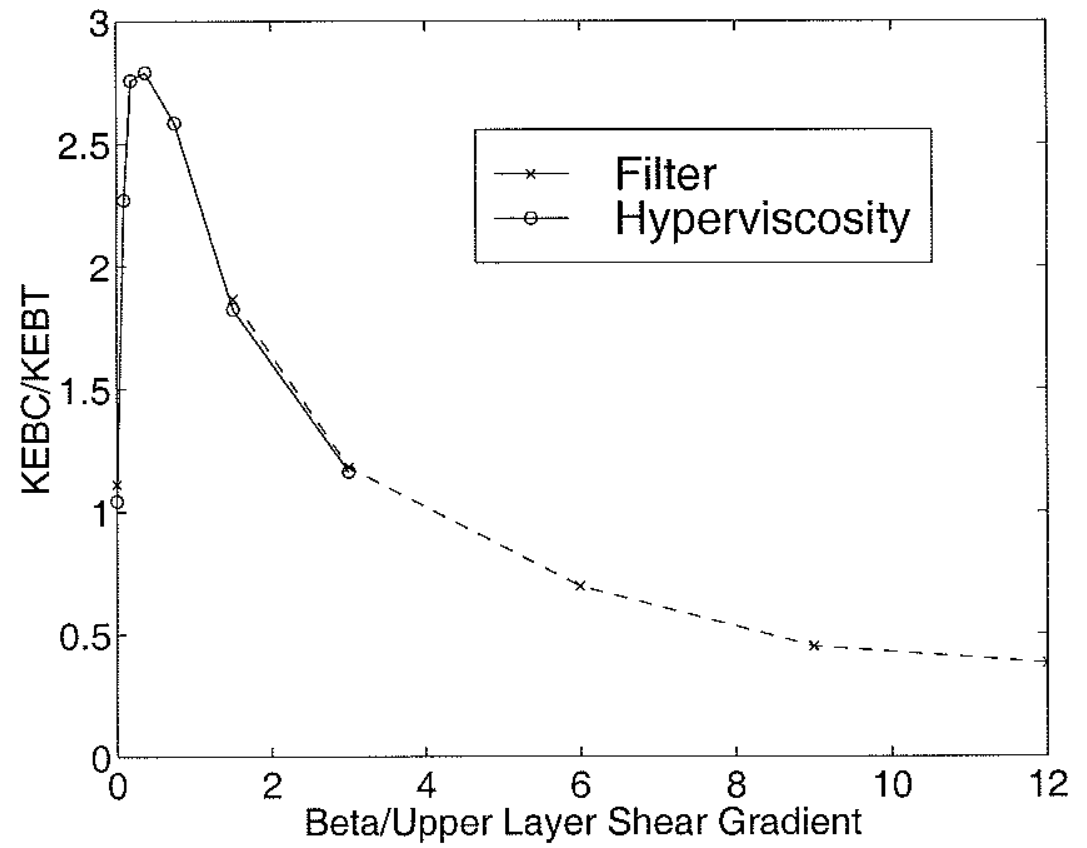

Figure 5-23: Modal kinetic energy partitions of experiments with fixed magnitude and angle of shear, but varying $R_{\beta}$. 


\section{Rbeta=12 (Filter)}

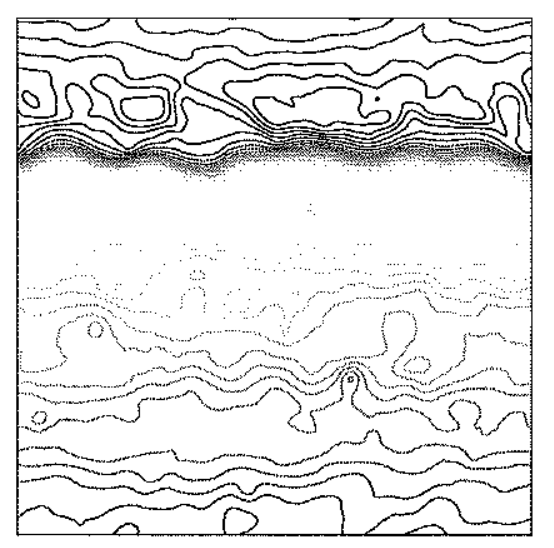

\section{Rbeta $=6$ (Filter)}

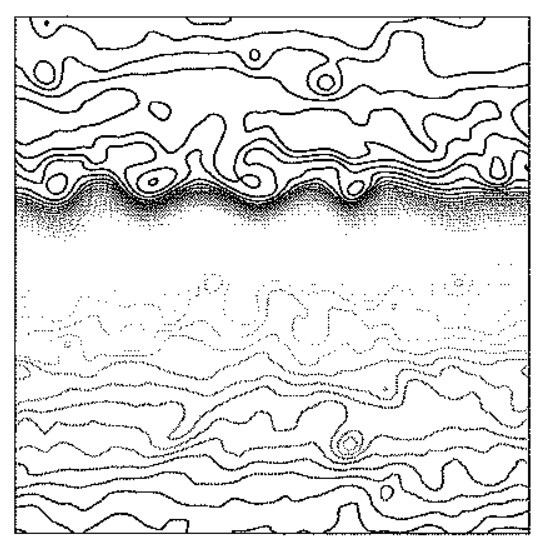

\section{Rbeta $=9$ (Filter)}

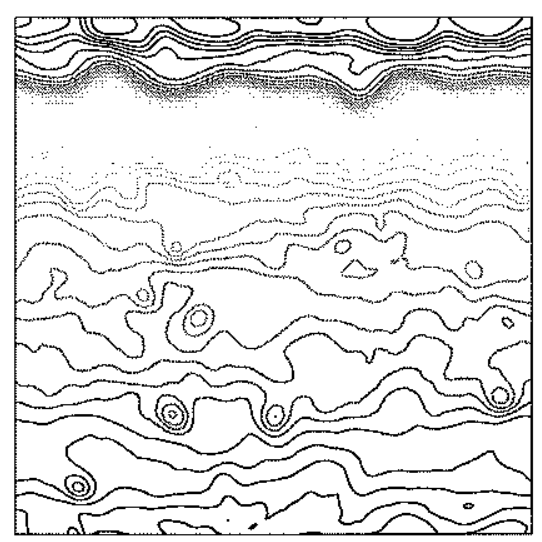

Figure 5-24: Contours of snapshots of upper layer fluctuation streamfunction for experiments with fixed magnitude and angle of shear, but varying $R_{\beta}$. In the large $R_{\beta}$ experiments, small-scale dissipation was accomplished with a wavenumber filter. 


\section{Rbeta $=3$ (Filter)}

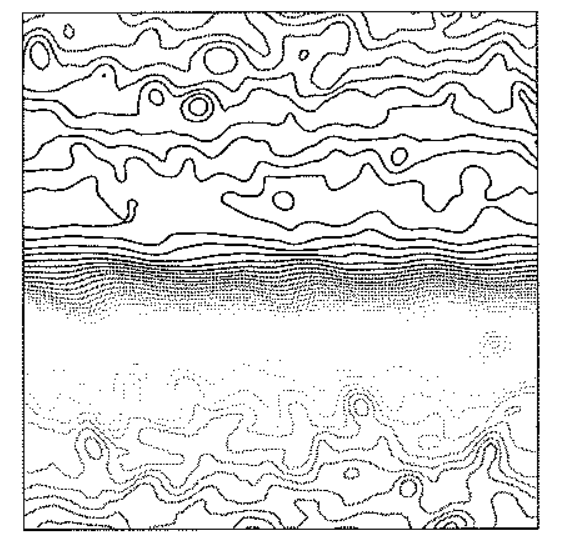

\section{Rbeta $=1.5$ (Filter)}

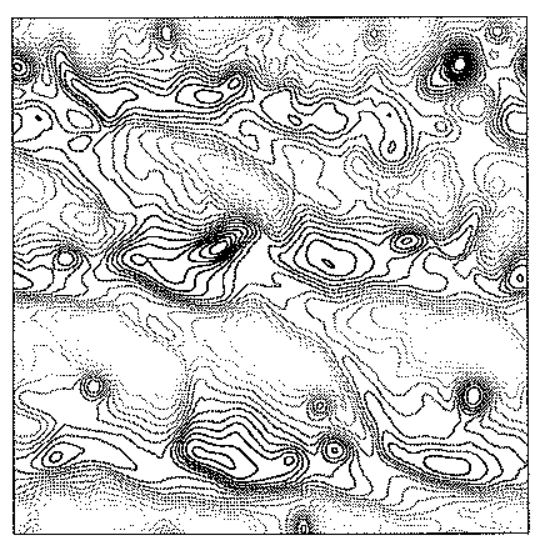

\section{Rbeta=3 (Hyper)}

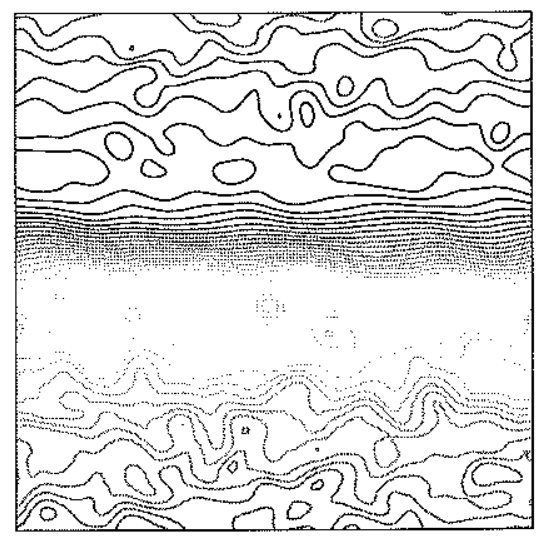

\section{Rbeta $=1.5$ (Hyper)}

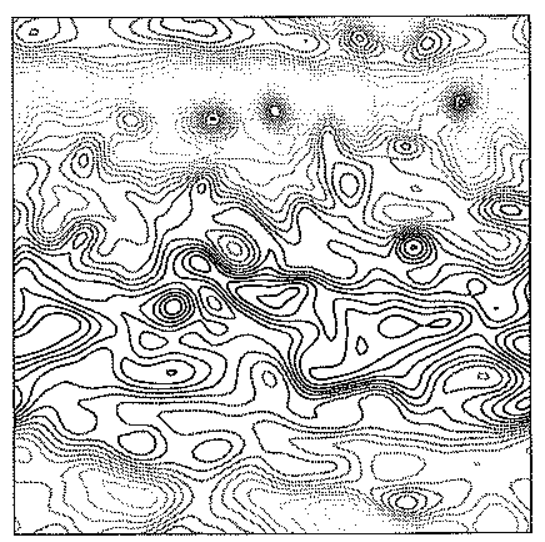

Figure 5-25: Contours of snapshots of upper layer fluctuation streamfunction for experiments with fixed magnitude and angle of shear, but varying $R_{\beta}$. In the medium $R_{\beta}$ experiments, both wavenumber filter and hyperviscosity were used as small-scale dissipations. The qualitative appearance of the two $R_{\beta}=3$ experiments is similar. This is less true for the two $R_{\beta}=1.5$ experiments. 
Rbeta $=0.75$ (Hyper)

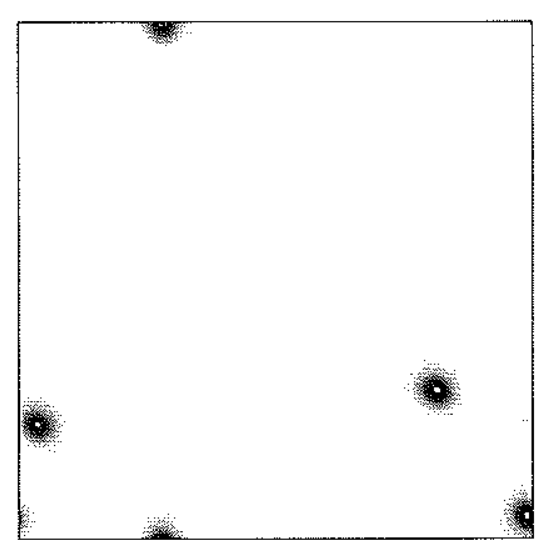

Rbeta $=0.1875$ (Hyper)

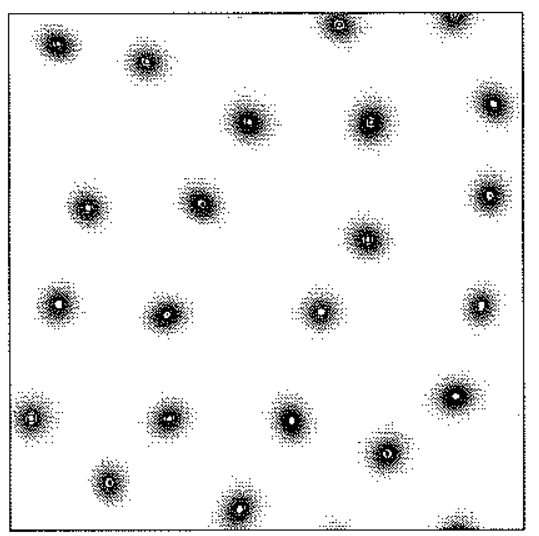

Rbeta $=0.375$ (Hyper)

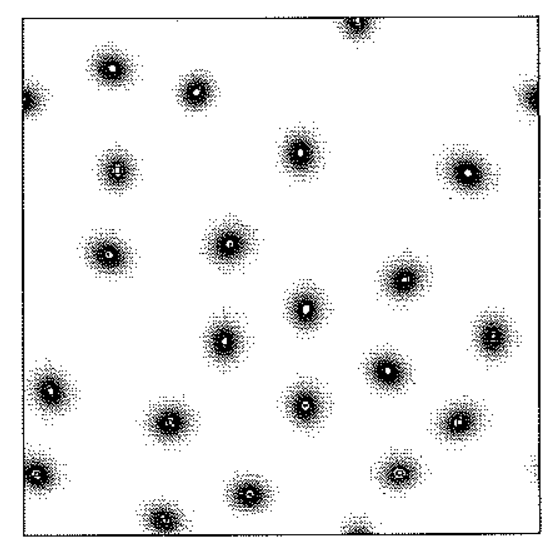

Rbeta $=0.09375$ (Hyper)

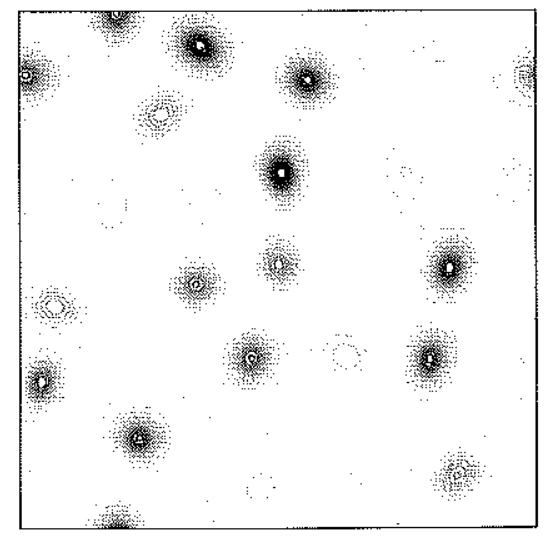

Figure 5-26: Contours of snapshots of upper layer fluctuation streamfunction for experiments with fixed magnitude and angle of shear, but varying $R_{\beta}$. When $R_{\beta}$ is less than one, small-scale dissipation was accomplished with hyperviscosity. 


\section{f-plane (Filter)}

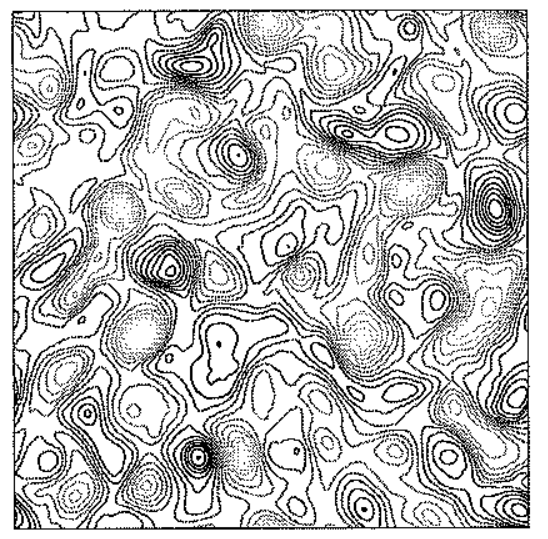

f-plane (Hyper)

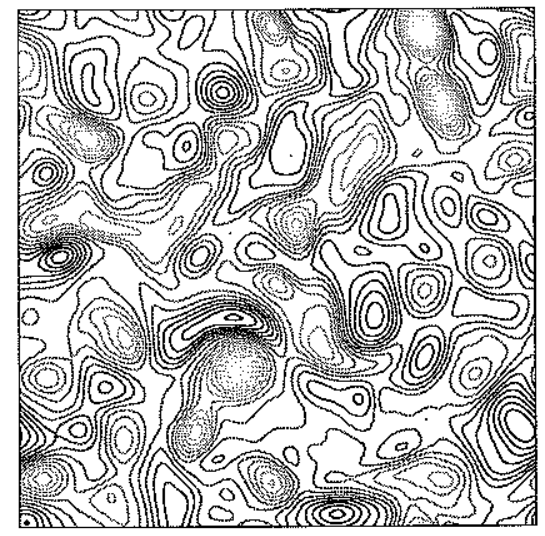

Figure 5-27: Contours of snapshots of upper layer fluctuation streamfunction for f-plane experiments with both types of small-scale dissipation. The eddy fields are qualitatively similar.

Linear growth rates for the experiments with $R_{\beta}=12$ and $R_{\beta}=0.375$ are plotted in figure 5-28. The anisotropy present in the linear $R_{\beta}=12$ plot (note the different $\mathrm{x}$ and $\mathrm{y}$ scales) is evident in the spinup phase as well, which is contoured in figures 5-29 through 5-33. The spinup is more complex than the f-plane spinup shown in chapter 3 , and proceeds through several stages. 

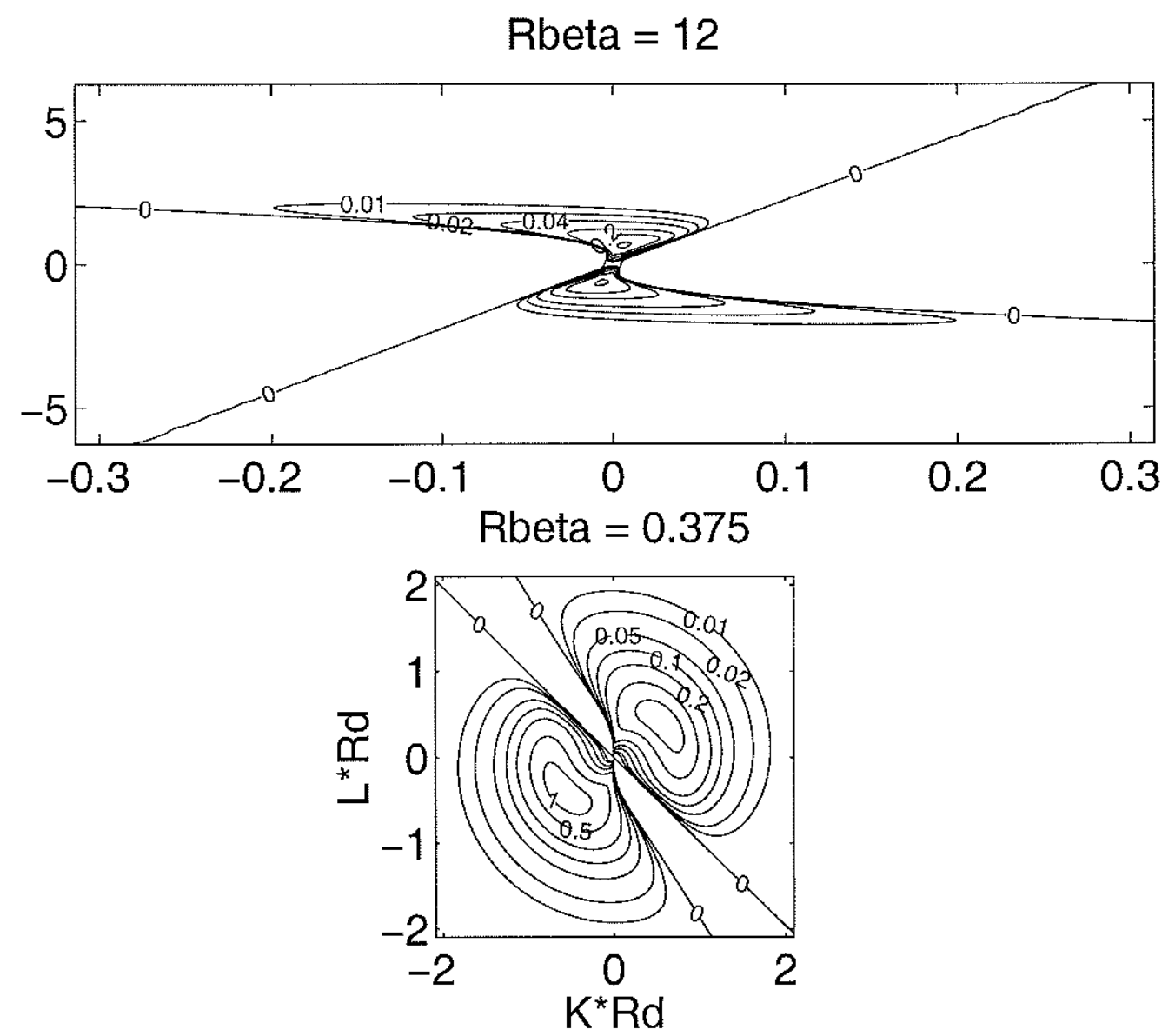

Figure 5-28: Linear growth rates in units of $10^{-8} \mathrm{~s}^{-1}$, for a nonzonal 210 degree flow having $R_{\beta}=12$ (top panel) and $R_{\beta}=0.375$ (bottom panel). 


\section{Initial Psi1}

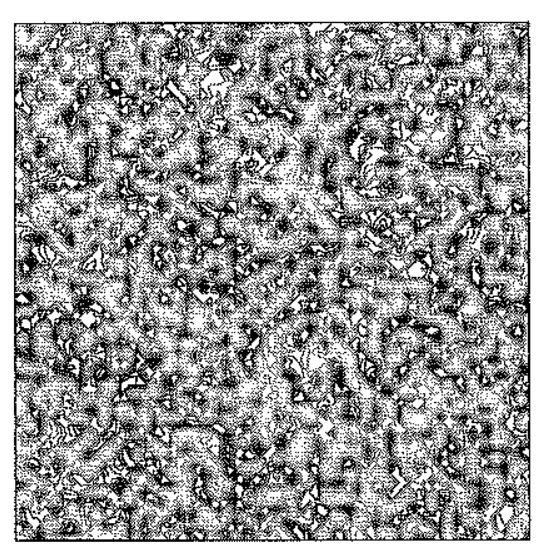

\section{7 years}

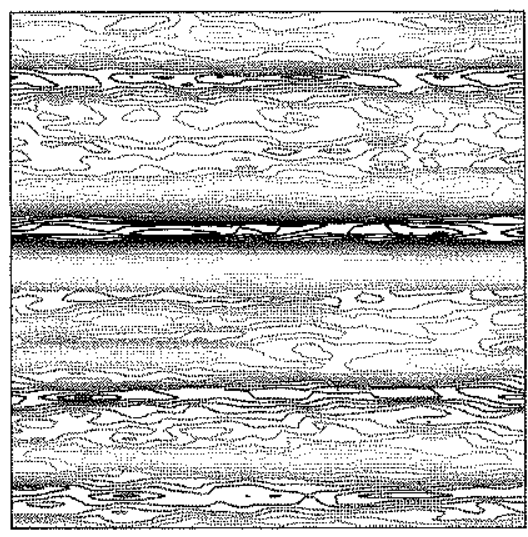

\section{9 years}

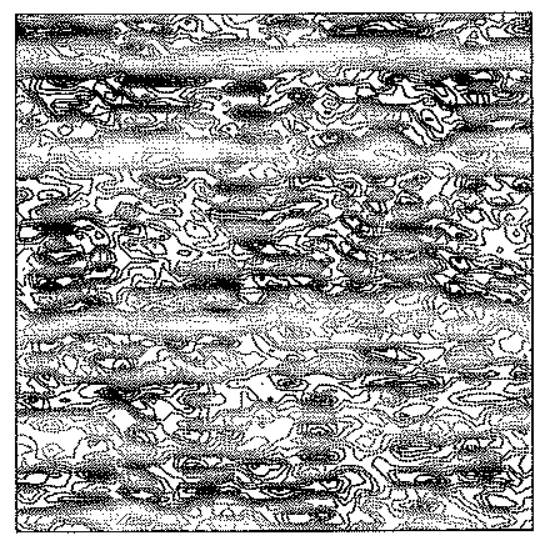

38.8 years

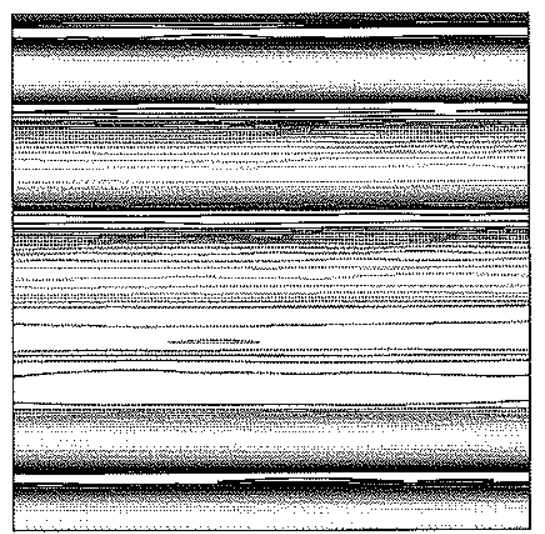

Figure 5-29: Contours of upper layer fluctuation streamfunction for the 64 squared spinup of nonzonal 210 degree experiment with $R_{\beta}=12$. 


\section{Psi1 50.8 years}

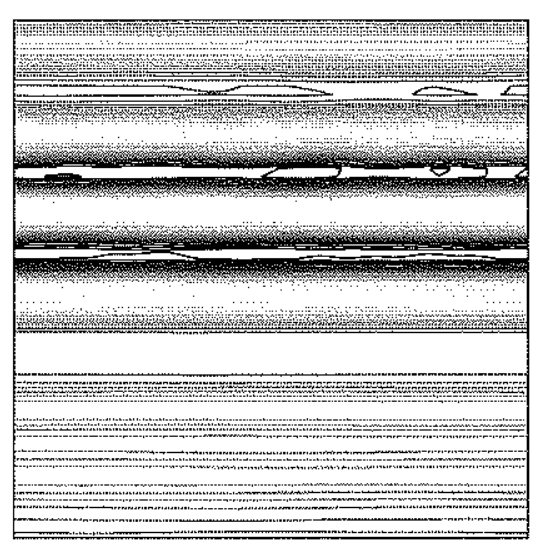

\section{Psi1 51.5 years}

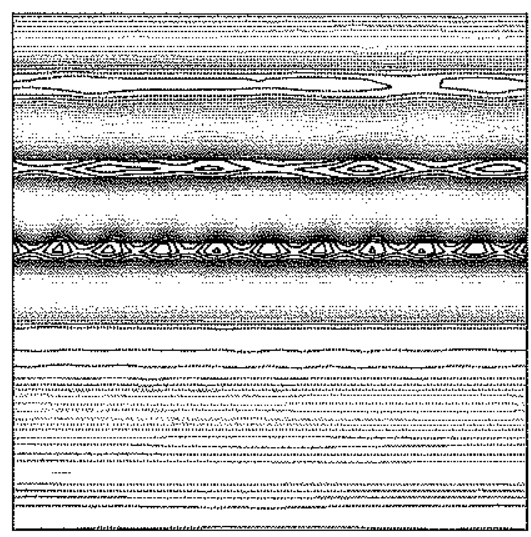

\section{Psi2 50.8 years}

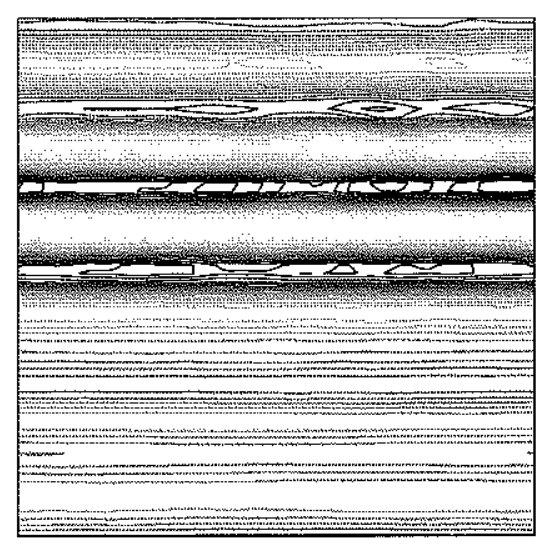

\section{Psi2 51.5 years}

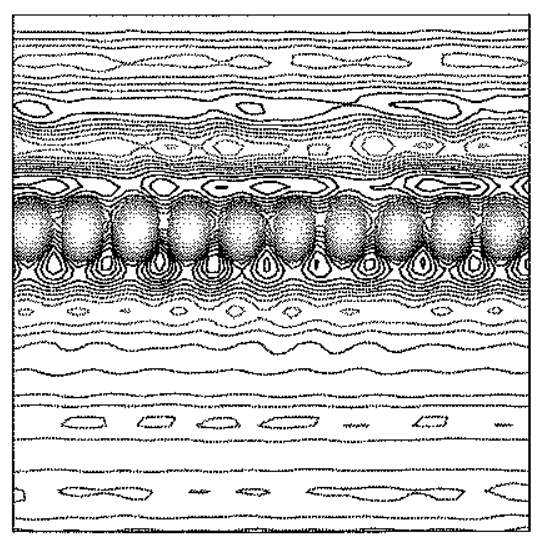

Figure 5-30: Contours of both layer fluctuation streamfunctions for the 64 squared spinup of nonzonal 210 degree experiment with $R_{\beta}=12$. 


\section{Psi1 52.1 years}

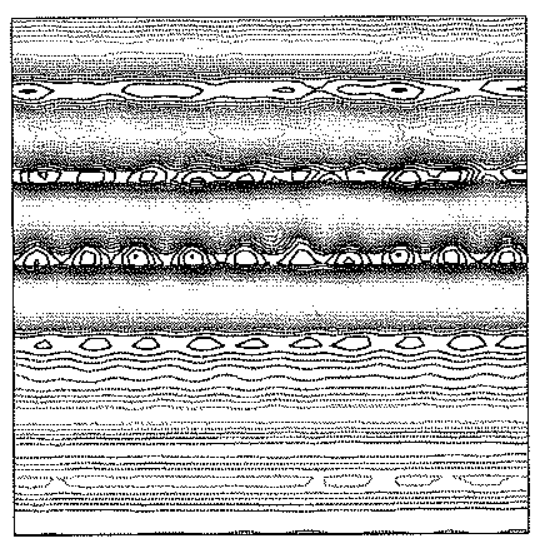

\section{Psi1 54.0 years}

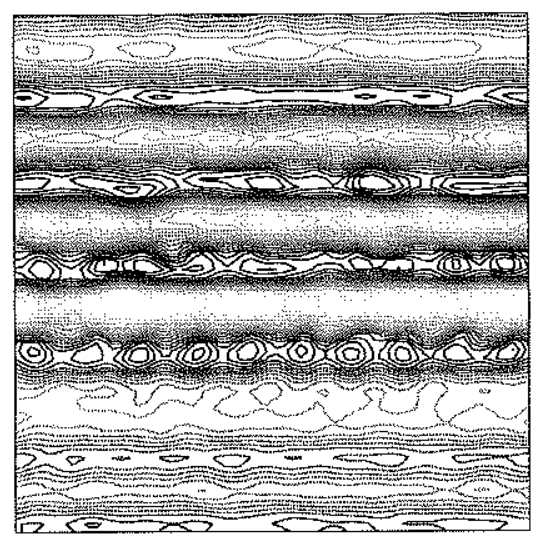

\section{Psi2 52.1 years}

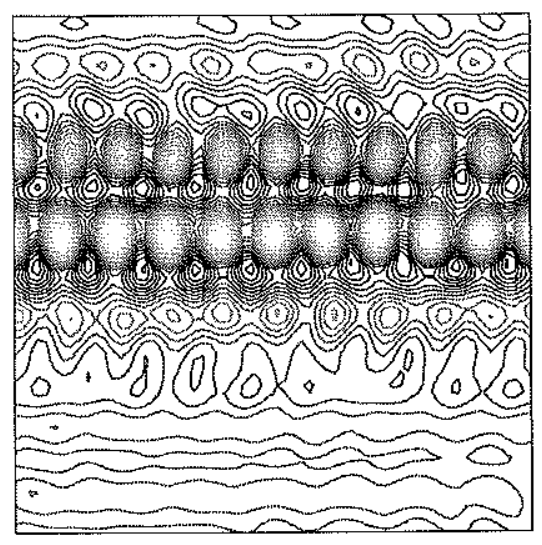

Psi2 54.0 years

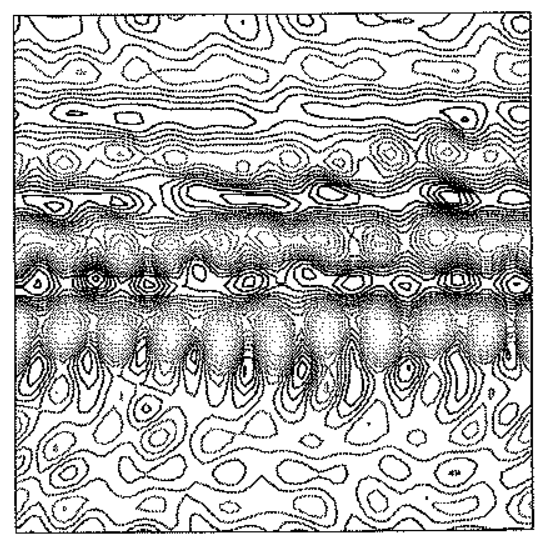

Figure 5-31: Contours of both layer fluctuation streamfunctions for the 64 squared spinup of nonzonal 210 degree experiment with $R_{\beta}=12$. 


\section{8 years}

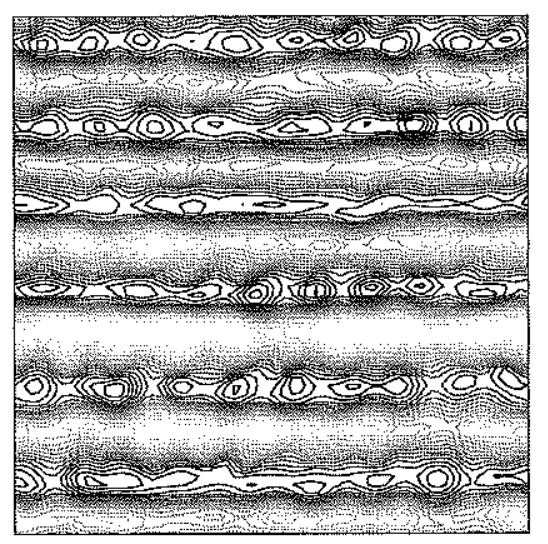

\section{0 years}

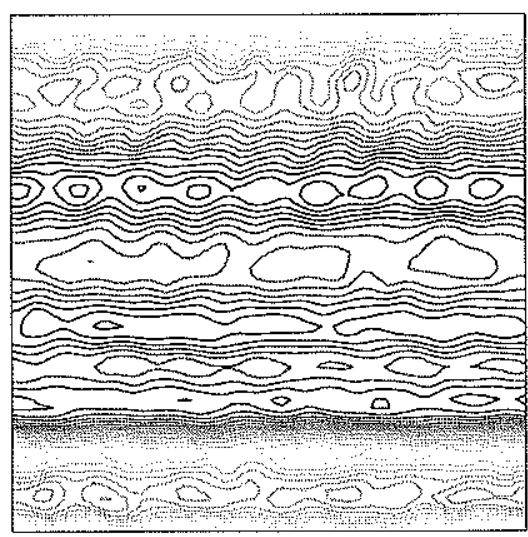

158.2 years

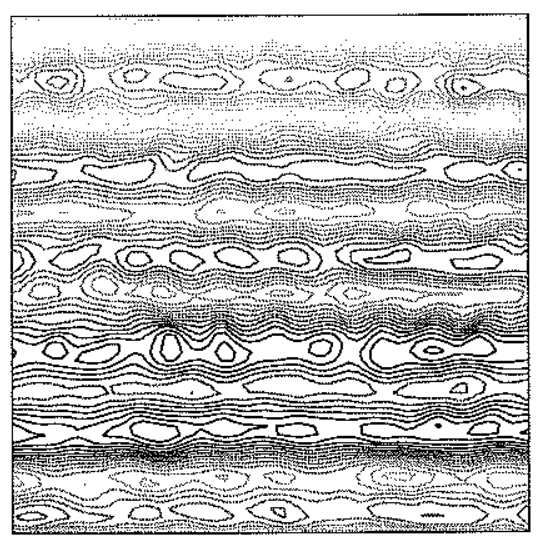

221.8 years

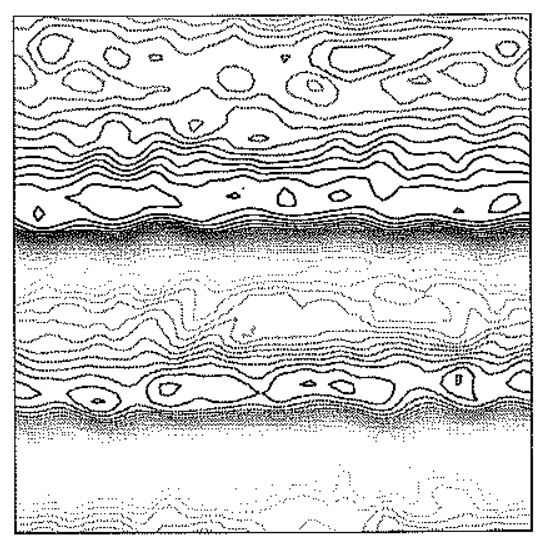

Figure 5-32: Contours of upper layer fluctuation streamfunction for the 64 squared spinup of nonzonal 210 degree experiment with $R_{\beta}=12$. 


\section{7 years}

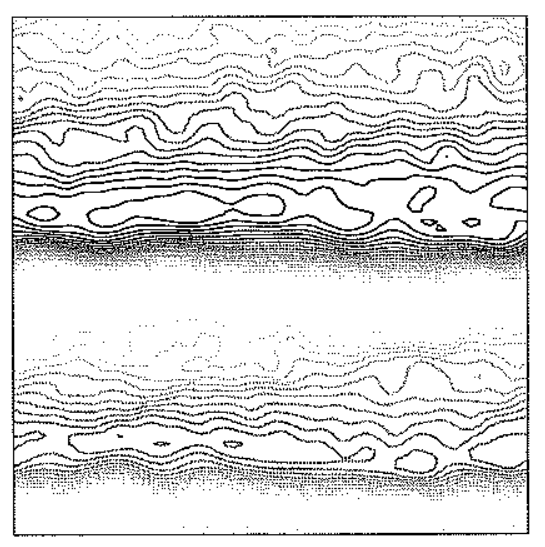

\section{1 years}

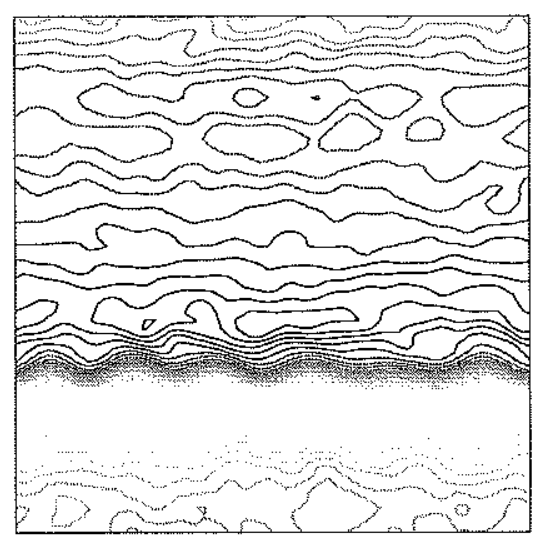

244.0 years

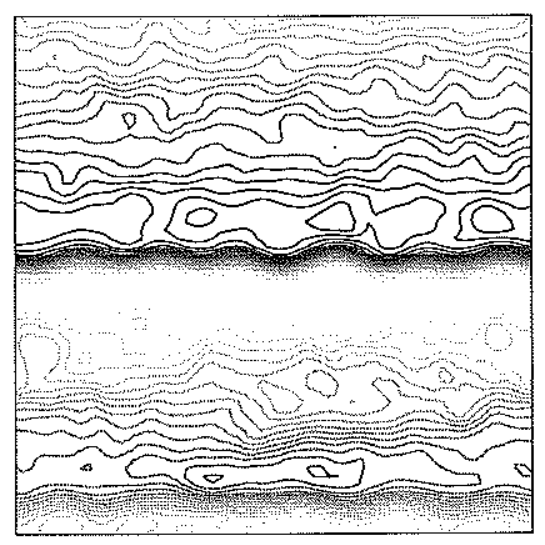

Final Psi1

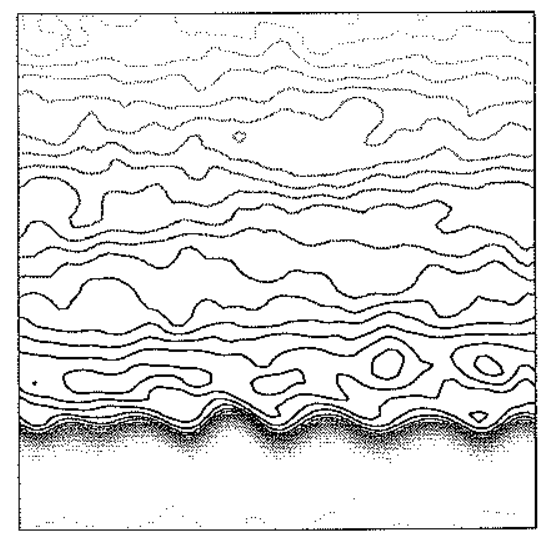

Figure 5-33: Contours of upper layer fluctuation streamfunction for the 64 squared spinup of nonzonal 210 degree experiment with $R_{\beta}=12$. 
Energy is plotted versus time during the spinup phase of the $R_{\beta}=12$ experiment in figure 5-34. The plot is marked at five spots of interest, all of which have been contoured above. From a small-scale random initial condition, eddy energy reaches a minimum at 8.9 years (the first line in figure 5-34). At this point hints of anisotropy can already be seen. Energy then undergoes its steepest growth, as waves varying in the north-south direction (which grow fastest) continue to be selected out of the random initial condition. By the time the waves are strong enough to generate secondary instabilities, at 50.8 years (the second line in figure 5-34), both kinetic and total energies are already larger than those of the fully equilibrated f-plane case. Now we can give a rough explanation of why equilibrated eddy energies are larger for the same magnitude of shear when there is a strong beta present than on an f-plane. The anisotropy of the former implies that solutions that are nearly linear waves will be selected from a random initial condition. These waves grow exponentially for a long time before processes which eventually lead to equilibration begin to develop.

At around 50.8 years, development of secondary instabilities is rapid. The next four snapshots show streamfunctions in both layers. Secondary instability is present in the bottom layer as well as the top. Energy still increases beyond 50.8 years, but at a much slower rate. Let us see if we can explain this transition point through calculation of the total PV gradients (imposed background plus fluctuations) in the two layers:

$$
\begin{aligned}
& \frac{\partial q_{\text {total-layer } 1}}{\partial y}=\beta+\frac{\left(\overline{u_{1}}-\overline{u_{2}}\right)}{(1+\delta) R_{d}^{2}}+\frac{\partial q_{1}}{\partial y}, \\
& \frac{\partial q_{\text {total-layer } 2}}{\partial y}=\beta+\frac{\delta\left(\overline{u_{2}}-\overline{u_{1}}\right)}{(1+\delta) R_{d}^{2}}+\frac{\partial q_{2}}{\partial y} .
\end{aligned}
$$

We consider the waves together with beta and the imposed mean flow to define a new mean zonal basic state, from which secondary instabilities may grow. Figure 5-35 shows the zonally averaged total PV gradients at time 50.8 years. The waves have 
indeed induced a reversal of potential vorticity gradient in the upper layer, which apparently explains the appearance of secondary instabilities. Secondary instabilities of strongly zonal wave motions has been previously investigated by Pedlosky (1975b; 1975c). He found that they could act as an equilibration mechanism for waves growing on a zonal mean flow. In the current experiments, energy increases many times over after secondary instabilities develop and before equilibration is reached.

By 78.8 years (the third line in figure 5-34), the upper layer streamfunction has developed into alternating bands of jets (twelve of them) and small eddies. Then, slowly, a concentration of contours into fewer jets takes place. By 244.0 years (the fourth line in figure 5-34), two jets are present. After 420.1 years (the fifth and last line in figure 5-34), only one jet remains.

Figure 5-36 plots kinetic energy versus time for experiments with the four largest values of beta. It focuses on the transition from steep growth to slower growth, which takes place at the beginning of the spinup. We assume that this occurs when $\frac{\partial q_{\text {total-layer } 1}}{\partial y}$ changes sign, and use this to predict the transition points of the other three experiments using results from the $R_{\beta}=12$ experiment. The fluctuation term in equation 5.18 is linearly proportional to the fluctuation velocities (or derivatives of the fluctuation velocities). Thus, the hypothesis that potential vorticity gradients should change sign for secondary instabilities to develop, would lead us to believe that the fluctuation energies at the transition points for the different experiments should change as the square of $\left(\beta+\frac{\overline{u_{1}}-\overline{u_{2}}}{(1+\delta) R_{d}^{2}}\right)$. In the top left corner of figure 5-36 a line is drawn at the 50.8 year kinetic energy level for the $R_{\beta}=12$ experiment. Similar "turnover" points in the other experiments are predicted by taking that value of kinetic energy and multiplying by the square of the ratio of beta plus the top layer shear gradient. The figure indicates that this reasoning seems to work quite well for the $R_{\beta}=9$ and $R_{\beta}=6$ experiments, and somewhat less well for the $R_{\beta}=3$ experiments. We take this figure as further evidence that secondary instabilities 
arise when the zonal waves forced by the mean flow become strong enough to reverse $\frac{\partial q_{\text {total-tayer } 1}}{\partial y}$, and that this transition delineates the period of steepest growth from that of less steep growth. The transition from linear waves to a secondary instability regime occurs at higher energy when beta is increased. This provides a tentative answer as to why the energy of nonzonal experiments increases with increasing beta, where beta is large. The energy at the transition point is still far from the final equilibrated energy (figure 5-34), however, so it would be more satisfactory to have a theory for the fully equilibrated energy levels. In the opinion of Panetta (1993), development of such a theory will not be easy for jet-dominated regimes.

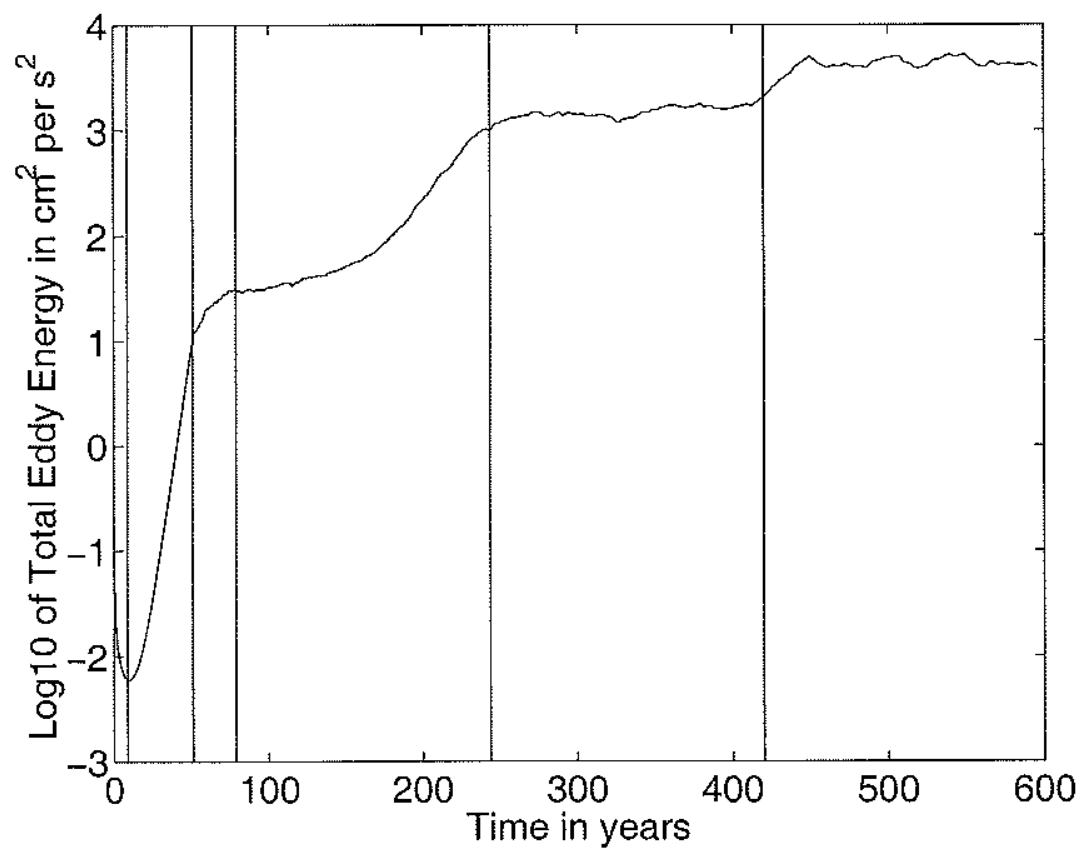

Figure 5-34: Spinup of 64 squared nonzonal 210 degree $R_{\beta}=12$ experiment. Lines drawn at $8.9,50.8,78.8,244.0$, and 420.1 years. Note that the experiment was continued at 256 squared resolution.

Energy versus time during the spinup of the $R_{\beta}=0.375$ experiment is plotted in figure 5-37, and the spinup is contoured in figures 5-38 through 5-40. The spinup is more isotropic. Eddy energy reaches a minimum more quickly here, at around 1.59 years, most likely because of the isotropy; in the $R_{\beta}=12$ experiment a smaller 


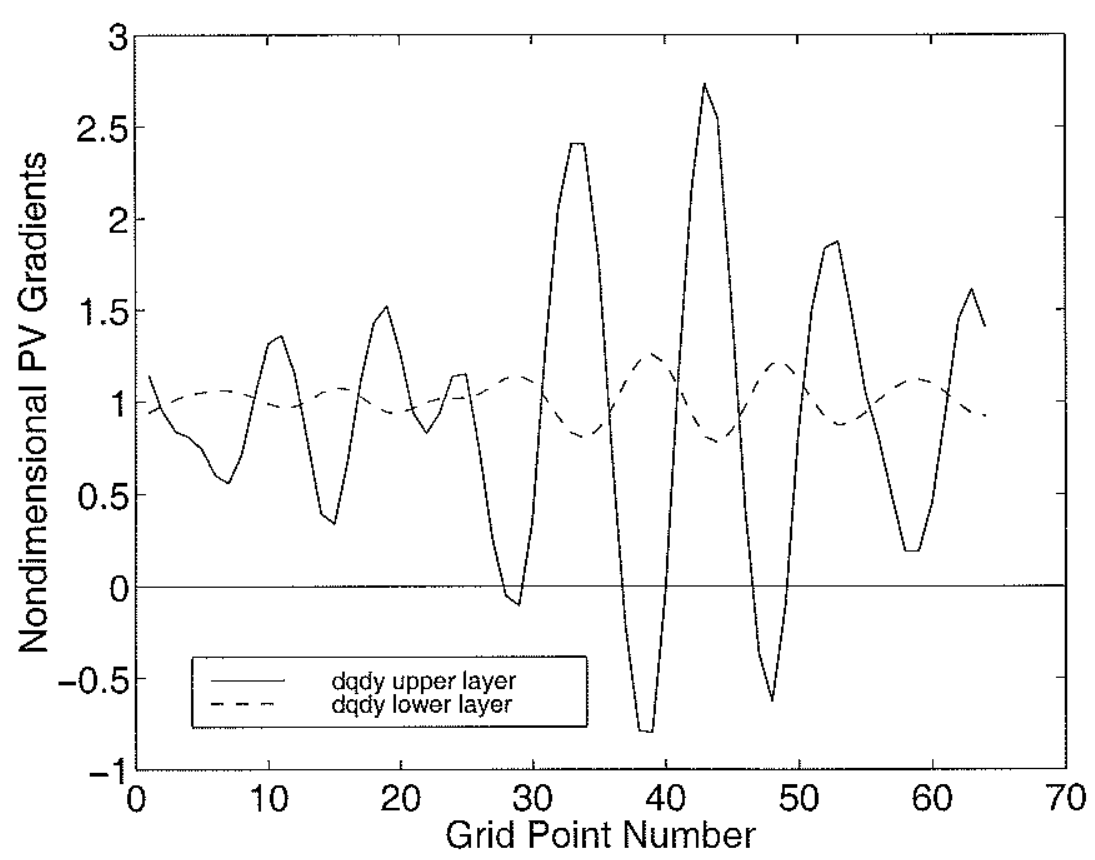

Figure 5-35: Zonally averaged total meridional PV gradient, at time 50.8 years of 64 squared nonzonal 210 degree $R_{\beta}=12$ experiment.

band of wavenumbers were unstable. Between 11.9 and 31.0 years energy briefly undergoes a period of smaller growth, before increasing again. Figure 5-41 demonstrates that small amounts of cyclone/anticyclone asymmetry are present during this period. By 40.5 years (figure 5-42), asymmetry is clear, and by 58.8 years (figure 5-43), asymmetry is very strong (all the eddies are cyclones). We will examine this asymmetry more carefully in the next chapter. 

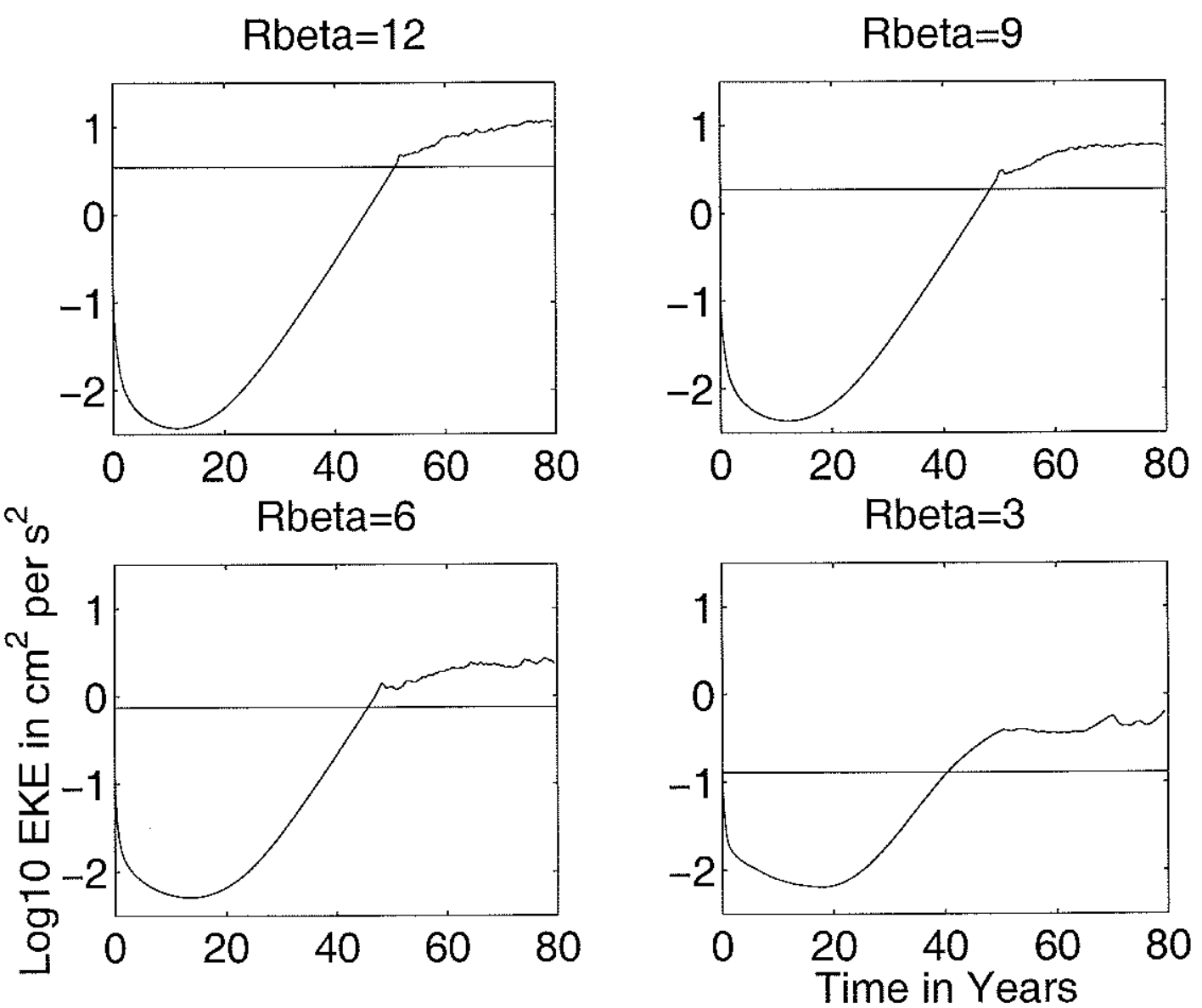

Figure 5-36: Transition from linear waves to secondary instabilities for 64 squared nonzonal 210 degree experiments having different values of $R_{\beta}$ greater than one. Extra lines indicate transition points predicted from the $R_{\beta}=12$ experiment; see text for details. 


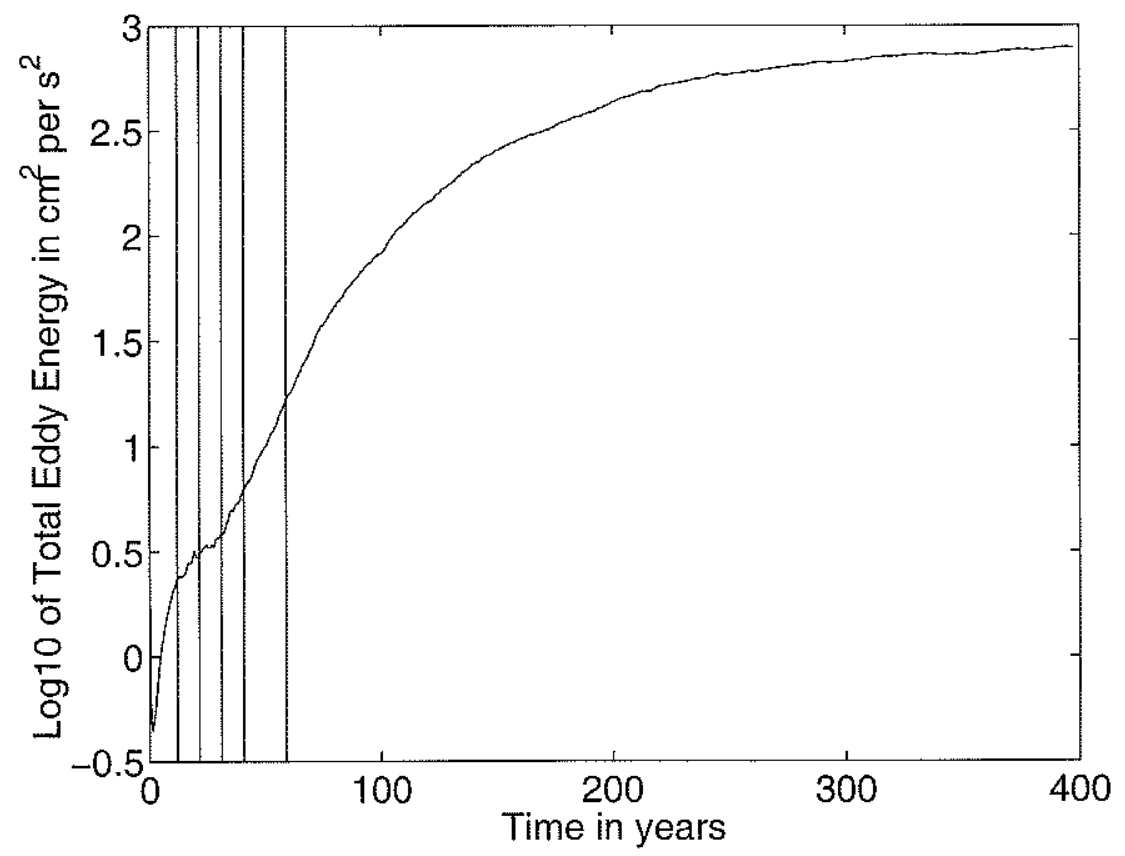

Figure 5-37: Spinup of 128 squared nonzonal 210 degree $R_{\beta}=0.375$ experiment. Lines drawn at $11.9,21.5,31.0,40.5$, and 58.8 years. Note that reaching final equilibration took a long time; the experiment was continued until about 1200 years. 


\section{Initial Psi1}

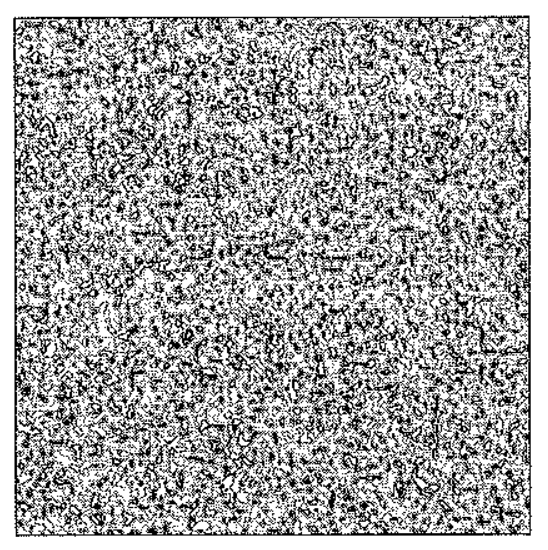

\subsection{7 years}

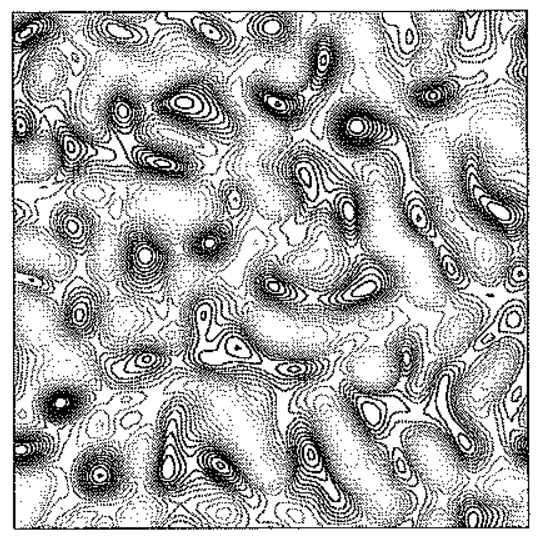

\subsection{9 years}

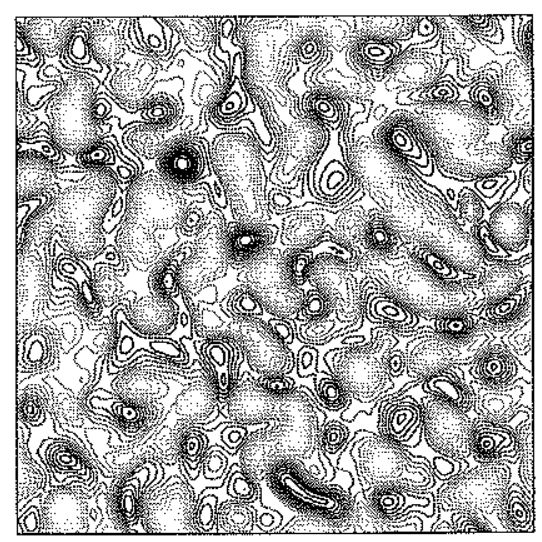

\section{9 years}

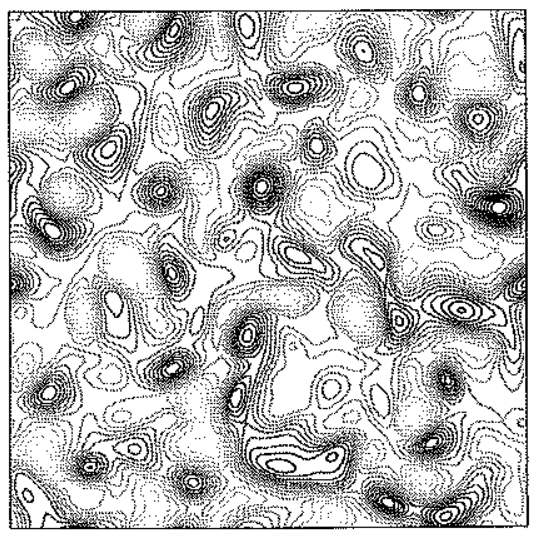

Figure 5-38: Contours of upper layer fluctuation streamfunction for the 128 squared spinup of nonzonal 210 degree $R_{\beta}=0.375$ experiment. 


\section{5 years}

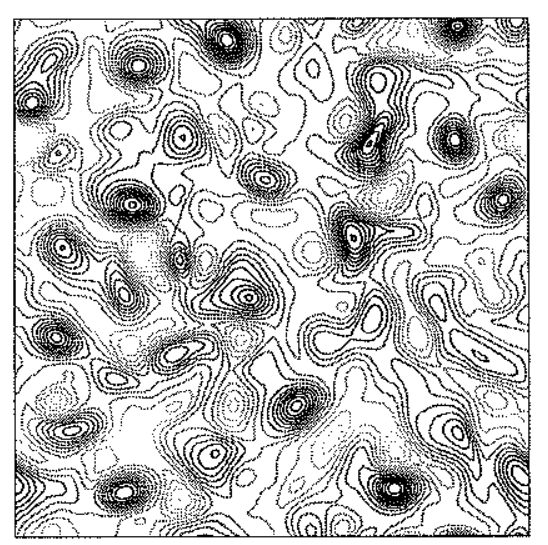

\section{0 years}

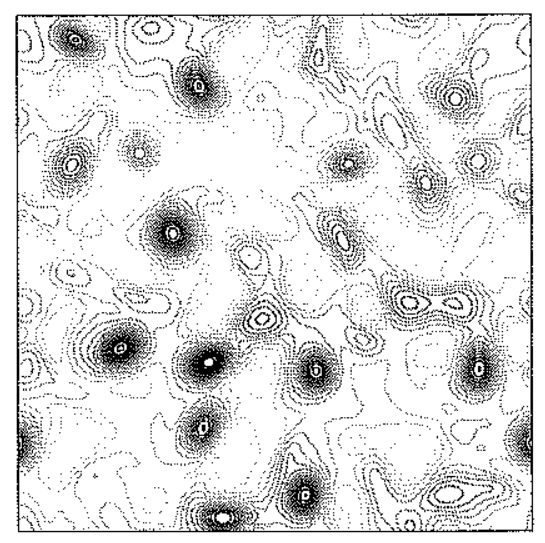

27.0 years

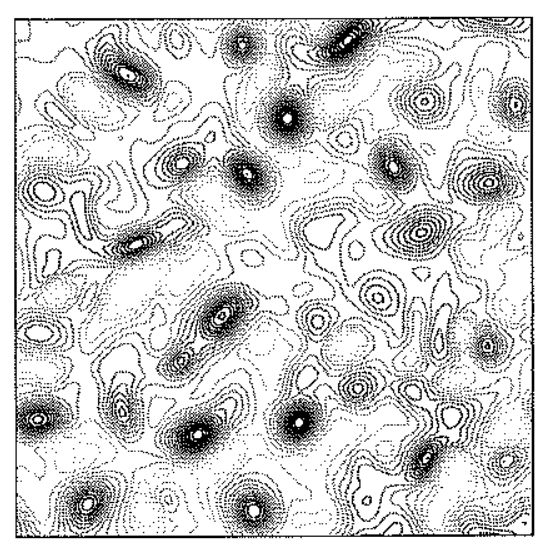

\section{5 years}

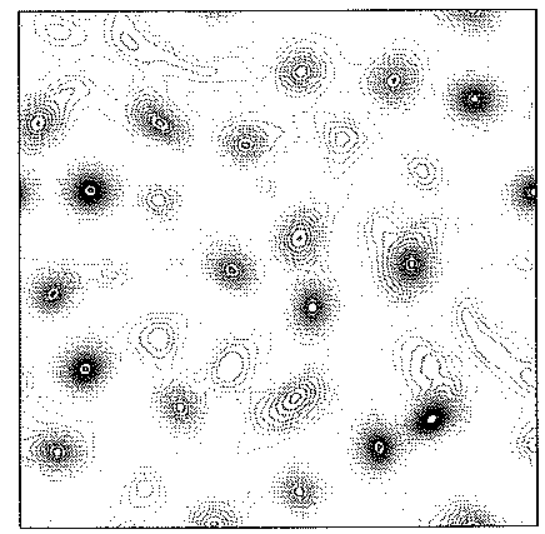

Figure 5-39: Contours of upper layer fluctuation streamfunction for the 128 squared spinup of nonzonal 210 degree $R_{\beta}=0.375$ experiment. 
58.8 years

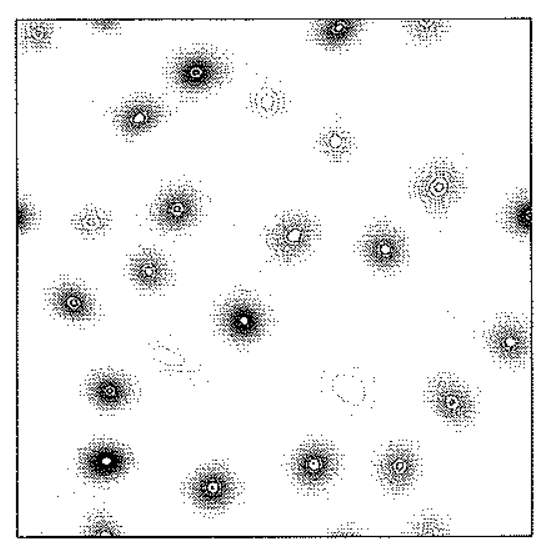

158.2 years

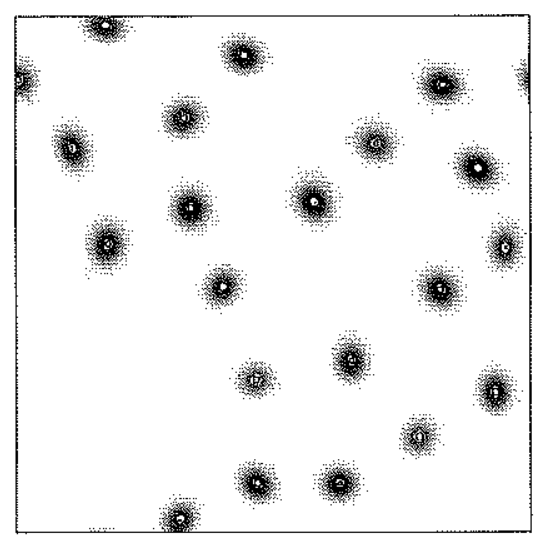

78.7 years

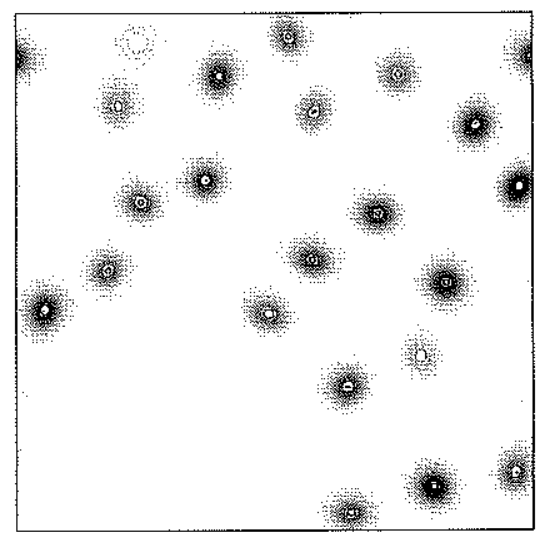

397.5 years

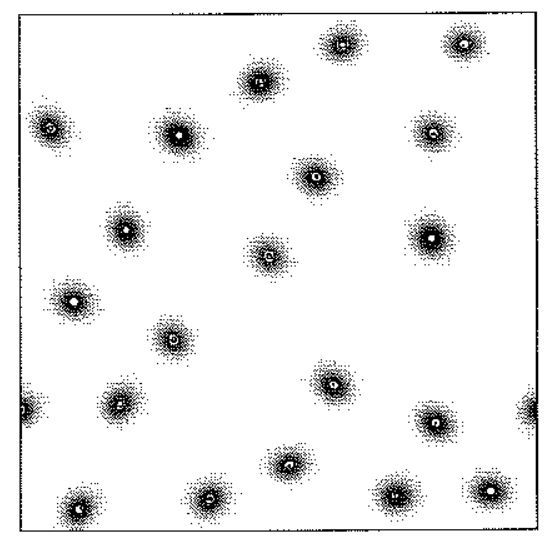

Figure 5-40: Contours of upper layer fluctuation streamfunction for the 128 squared spinup of nonzonal 210 degree $R_{\beta}=0.375$ experiment. 


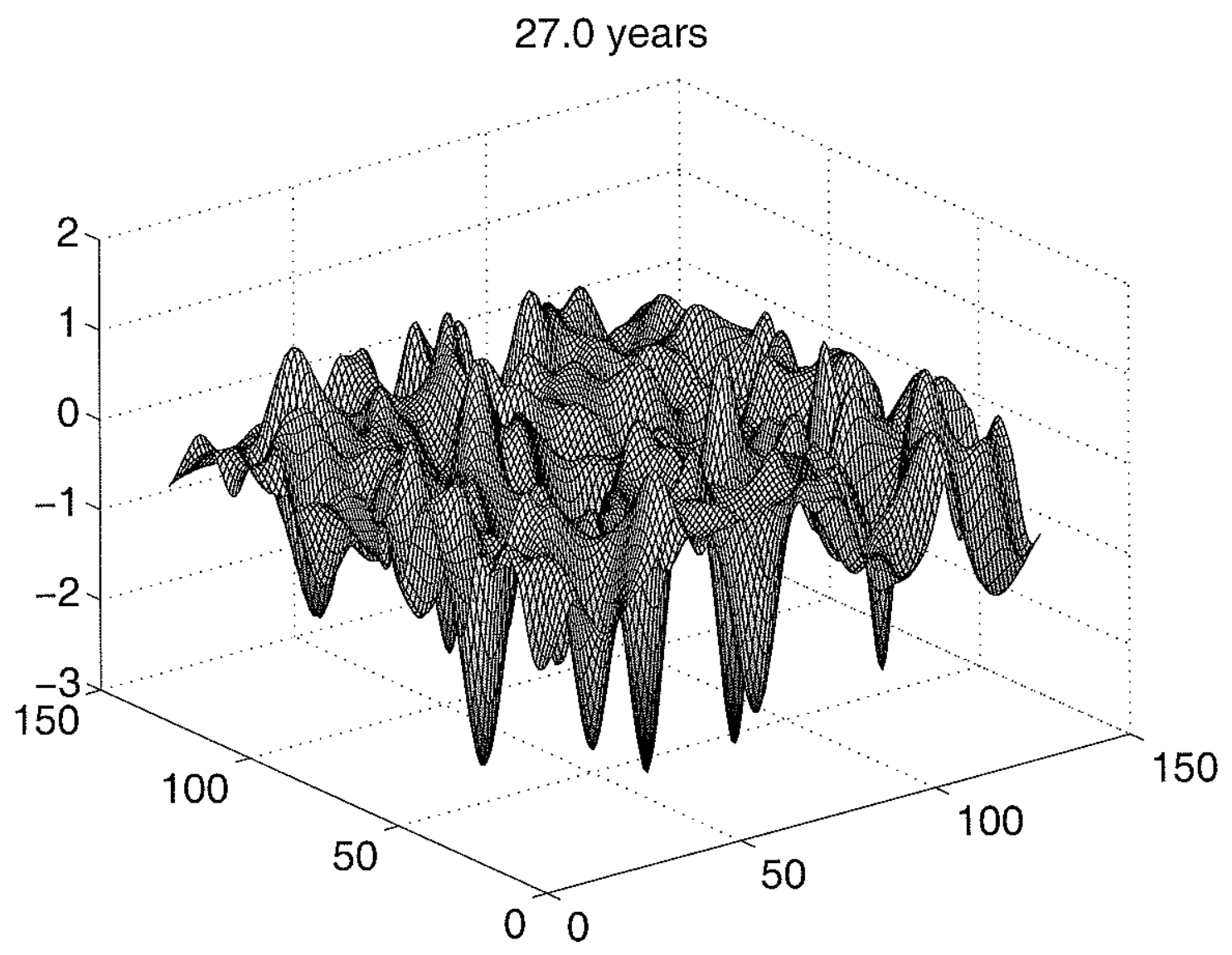

Figure 5-41: Surface plot of upper layer fluctuation streamfunction at 27.0 years of the 128 squared spinup of nonzonal 210 degree $R_{\beta}=0.375$ experiment. 


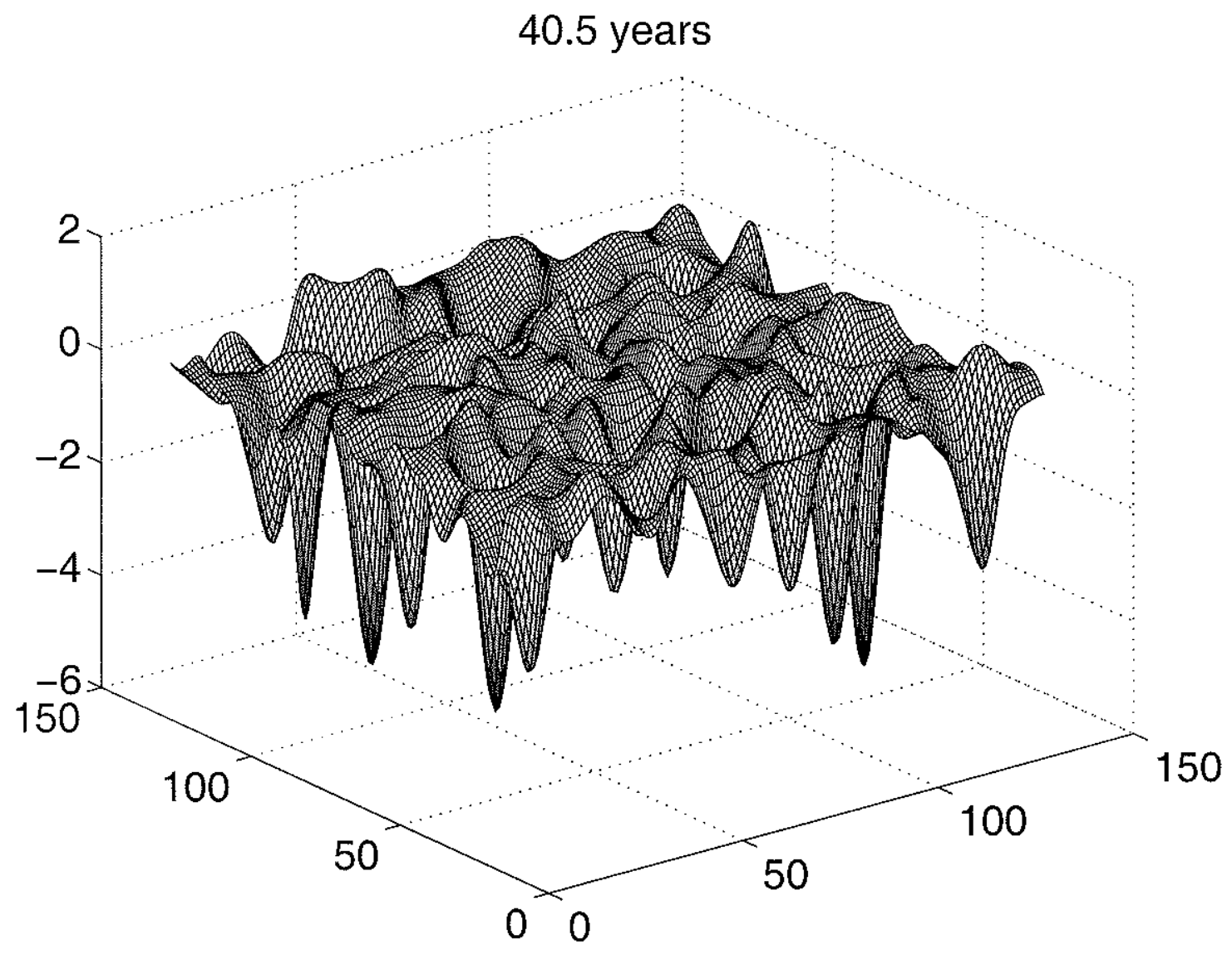

Figure 5-42: Surface plot of upper layer fluctuation streamfunction at 40.5 years of the 128 squared spinup of nonzonal 210 degree $R_{\beta}=0.375$ experiment. 


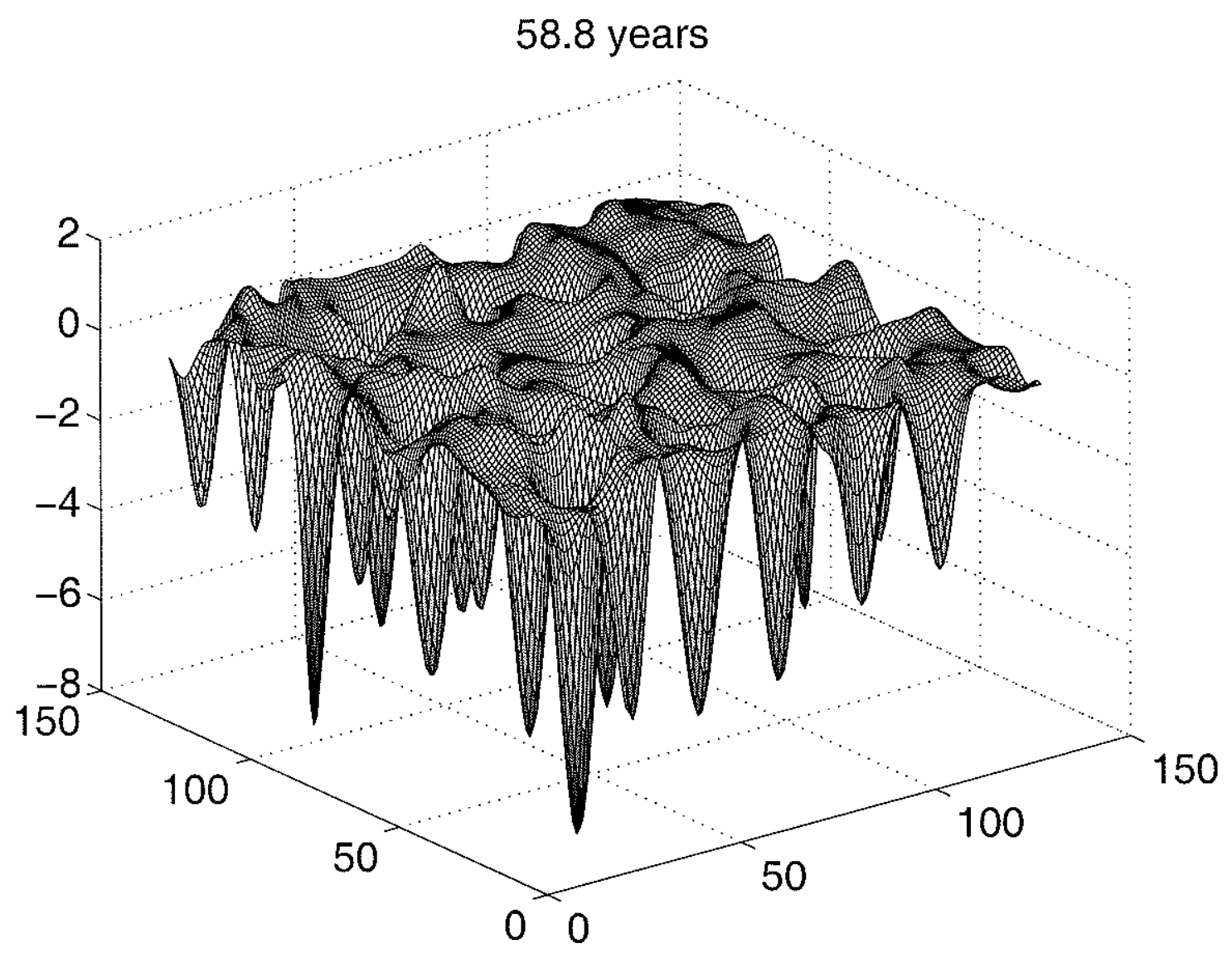

Figure 5-43: Surface plot of upper layer fluctuation streamfunction at 58.8 years of the 128 squared spinup of nonzonal 210 degree $R_{\beta}=0.375$ experiment. 


\subsection{Chapter summary}

Linear stability analysis indicates that weak shear flows typical of mid-ocean gyres are stabilized in the presence of planetary beta. It also indicates that flows having the same magnitude of shear, but oriented nonzonally, are unstable. Thus, the original goal for this part of the thesis was to show that the inherent nonzonality of gyre mean flows is an important factor in the production of mid-ocean eddies through local baroclinic instability. Before getting to the pertinent experiments, a few preliminary points regarding nonzonal flows were addressed. First, we discussed in a qualitative way differences in baroclinic instability arising from flows on an f-plane and from weak nonzonal flows on a beta plane. Of particular importance is the realization that the latter will be more anisotropic. Then we showed that $J(\bar{\psi}, \bar{q})=0$ can be satisfied for nonzonal flows on a beta plane. In pursuing that argument we found that mean PV gradients between layers are non-colinear if and only if planetary beta and meridional shears are present at the same time.

Following these preliminaries we did in fact show that for weak shear flows having a fixed value of beta, energy is a strong function of angle (and is actually too large when compared to observations). Therefore, those parts of the gyre that flow nearly in a north-south direction may generate the bulk of eddy energy in the interior. In the process of showing this, we encountered some unexpected results. Instantaneous snapshots of upper layer streamfunction and zonal averages of upper layer velocity reveal spatial inhomogeneities not present in the experiments of Panetta (1993), who forced jets through instability of zonal flows. In nonzonal experiments, for a wide variety of angles, a single front is formed, which then slowly drifts thoughout the domain, via advection by the mean meridional shear. The frontal width is not simply

related to the $\sqrt{\frac{U_{\text {eddy }}}{\beta}}$ scale. The appearance of inhomogeneity may have to do with the fact that zonal jets arising from a background flow with a meridional component actively extract energy from the background. Thus the preference of zonal motions due to the imposition of planetary beta may make equilibration impossible unless 
inhomogeneities arise so that eddy flux divergences can act on the jet.

All of the above experiments are more anisotropic than the actual mid-ocean eddy field. We anticipate that anisotropy can be reduced in doubly periodic nonzonal flow experiments through reduction of the ratio $R_{\beta}=\frac{\text { planetary beta }}{\text { upper layer shear gradient }}$. Then shear-induced PV gradients will be comparable to beta, and the angle between layer vorticity gradients will be larger, as seen in oceanic PV maps (figures 5-16 and 5-17). With this serving as motivation, the other set of experiments performed in this chapter investigated the effects of varying beta for a fixed angle and magnitude of shear. When $R_{\beta} \gg 1$, eddy fields are dominated by zonal jets. Their energy is greater than that of f-plane experiments having the same magnitudes of shear and friction. For likely similar reasons, energy of the nonzonal large $R_{\beta}$ experiments increases as $R_{\beta}$ increases. Spinup behavior of the large $R_{\beta}$ experiments points the way towards a tentative explanation of these facts. At first, selection takes place from a random initial condition of the zonal motions favored for exponential growth. When these zonal motions become strong enough, secondary instabilities develop. At this point, eddy energy has already surpassed that of the f-plane case. Energy continues to grow, but at a much slower rate, after the transition takes place. The secondary instabilities seem to be triggered by a reversal of total potential vorticity gradient in the top layer. As beta increases, so must the strength of the zonal motions, if the gradient is to be reversed. Hence, energy level at the transition increases with increasing beta (or $R_{\beta}$ ). This level is still far below the final equilibrated energy level, but the fact that it increases with beta, as does the final level, is encouraging. When $R_{\beta}$ is nonzero but less than one, eddy fields are indeed isotropic, and consist of monopolar vortices. These are the subject of the next chapter. 


\section{Chapter 6}

\section{Cyclone-anticyclone asymmetry in quasi-geostrophic turbulence forced by nonzonal mean flows}

This chapter focuses on the vortex regime uncovered in the last chapter. We begin with a discussion of their sensitivity to small-scale dissipation. From that will emerge hyperviscosity parameters and a vortex solution which we regard as nominal. Many of the other chapter results are based on slight alterations of the other parameters which determine the nominal solution. Dependence on strength of Ekman friction and angle of shear will be examined. All of the vortex solutions found up to this point will be fields of cyclones. Cyclone-anticyclone asymmetry will be documented in the governing equations, and initial conditions which produce anticyclones will be discussed. The anticyclones exist in a state of lower energy than do the cyclones, consistent with the asymmetries in the governing equations. Next, results of spin-down and freely-decaying experiments initialized with vortex solutions, in both equivalent barotropic and two layer systems, will be shown. The results provide tentative answers about maintenance of the vortices. After that, a determination of the dominant balance in the vortex regime will be made, which will provide more 
information on the dynamics of the vortices. A comparison to the coherent vortices of earlier studies will be made, followed by the chapter summary, which will discuss what we know and do not know about these vortices, as well as their possible significance.

\subsection{Sensitivity of vortex solutions to small-scale dissipation}

The vortex regime was originally found in 64 squared resolution experiments using the wavenumber filter described in chapter 2 (with an exponent of 7). Energy versus beta was plotted for nonzonal experiments at a fixed angle, as in figure 5-21, and a narrow peak was found for beta values that are small but nonzero. Inspection of the solution flow fields in this range revealed them to be vortical. Numerous exploratory runs, some of which will be shown here, were then performed at 64 squared resolution. Extension to higher resolution proved difficult. Starting from small scale random initial conditions, 128 squared and 256 squared simulations were attempted using a wavenumber filter. Vortices emerged at those resolutions (figure 6-1), but they did not equilibrate after a few months of processor time (figure 6-2). Number of vortices, as well as energy, increases dramatically with resolution. Clearly, a 64 squared domain provides marginal resolution in the vortex regime. Consistent with that, when the wavenumber filter exponent is changed to 4 at 64 squared resolution, the vortices disappear. Since the 128 squared and 256 squared wavenumber filter solutions would not converge in a resonable amount of time, it was decided to perform

high-resolution explorations of the vortex regime using hyperviscosity $(-1)^{n+1} \nu \nabla^{2 n} q$ as our small-scale dissipation. Some time was spent exploring different hyperviscous powers $n$ and coefficients $\nu$. The range of $\nu$ over which the vortices exist widens as $n$ increases, and also as resolution increases. We did not find vortices when $n$ was less than 3 . We chose $n=3$ and $\nu=0.0001$ (nondimensional) as our nominal 
hyperviscosity parameters. With these values, vortices emerge and equilibrate in a reasonable time-evidently some dissipation is needed at vortex scales $\left(\sim R_{d}\right)$ for rapid equilibration. The nondimensional Ekman damping rate was 0.03 for most of the vortex experiments (excepting the ones in which Ekman friction was varied). The length scale was nondimensionalized by the deformation radius; that is, $\mathrm{L}=1$ corresponds to $R_{d}$. Therefore, at a wavelength of $2 \pi R_{d}$, hyperviscous damping is 300 times less than Ekman damping. That our choice of hyperviscosity parameters is reasonable is confirmed in section 5.3 , in which we saw that three solutions outside of the vortex regime were little different whether hyperviscosity or wavenumber filter was used. A hyperviscous vortex solution is contoured alongside wavenumber filter results in figure 6-1 for the sake of comparison. Deviations from a circular state are more noticable in the hyperviscous solution (and in the 256 squared solution, which is presumably far from equilibrium). Solutions with both wavenumber filter and hyperviscosity clearly represent the same phenomenon. This hyperviscous experiment is taken to be our nominal vortex solution. It was continued at 256 squared resolution (but with $n$ and $\nu$ kept constant) with no difference in energy resulting. It was also run with the same shear vector but with lower layer mean velocities set to zero, so that $J(\bar{\psi}, \bar{q})=0$ is no longer satisfied in the layers. The latter solution actually contained about twenty percent less energy, but was otherwise very much the same (in a statistical sense). Thus, an unbalanced mean flow does not add energy to the solution, and the vortices are not an artifact of the procedure we use to balance the mean flows.

Because vortices are more robust as resolution increases with a wavenumber filter, because they exist under both wavenumber filter and hyperviscosity, and because they exist for a wider range of coefficients as the power $n$ of hyperviscosity increases, we believe that they are not numerical artifacts. Vortex energy is more sensitive to the details of resolution and small-scale dissipation than are the other results put forth in this thesis. However, qualitative behaviors we will describe 
for the 128 squared hyperviscous experiments are very similar to those in the 64 squared wavenumber filter experiments. Hence there is hope that qualitative, if not quantitative, results are independent of small-scale dissipation.

\subsection{Dependence of vortex solutions on other pa- rameters}

The vortices were discovered in plots of energy versus beta for a fixed magnitude and angle of shear. For the 128 squared hyperviscous solutions, this plot was shown in the last chapter. So we have already looked at the dependence on beta to some extent. The criterion for vortices to exist on a beta plane appears to be that beta be less than the magnitude of the shear-induced PV gradient. Vortex size in figure 5-26 changes little as beta varies. The size of the vortices in $6-1$, which share the same value of beta, is also very nearly the same, despite the fact that energies vary greatly.

These facts indicate that vortex sizes are not set by the Rhines scale $\sqrt{\frac{V_{\text {eddy }}}{\beta}}$, contrary to conventional thinking about geostrophic turbulence on a beta plane. To further illustrate this point, we note that the Rhines scale in the upper layer is $\sim 18 R_{d}$ for the 64 squared wavenumber filter vortex experiment, which when multiplied by $2 \pi$ is larger than domain size.

A basic state consisting of a zonal $1 \mathrm{~cm} \mathrm{~s}^{-1}$ shear flow on an f-plane, with a weak linear slope topography varying in the east-west direction, also produces vortices (figure 6-3). This state has non-colinearity of layer PV gradients in common with the nonzonal beta plane experiments. The existence of vortices in the upper layer despite the lack of a beta there is further evidence of the irrelevance of the Rhines scale in the vortex regime; in this case, there is no Rhines scale in the upper layer. The topographic results cloud the issue of what determines the transition into a vortex regime, since in that case the upper layer shear-induced potential vorticity gradient cannot be compared to a planetary beta. No other topographic results will 
64 squared

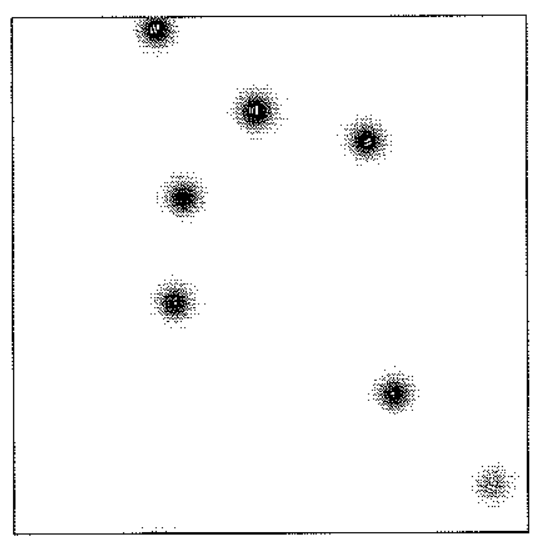

\section{6 squared}

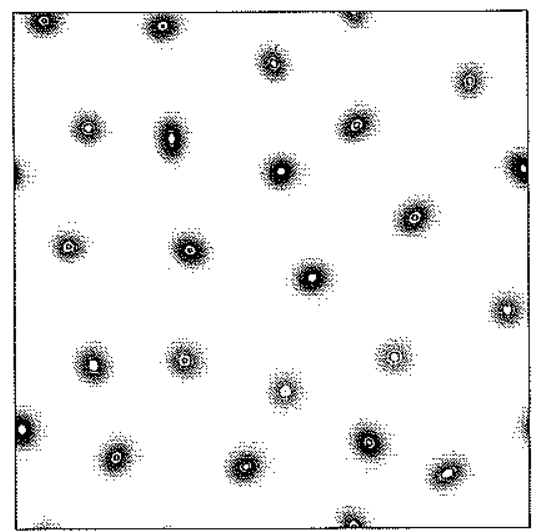

128 squared

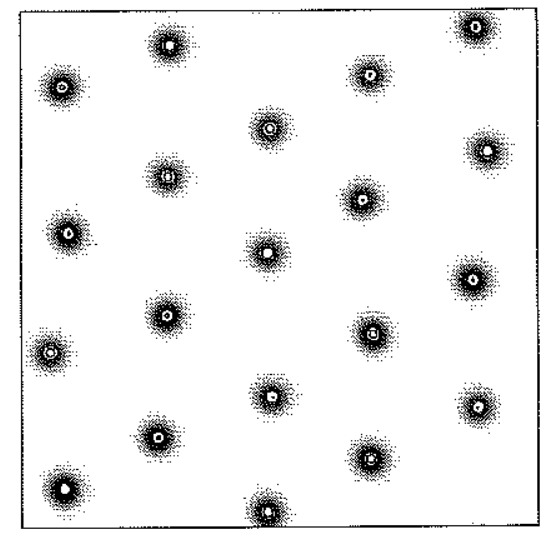

128 squared hyper

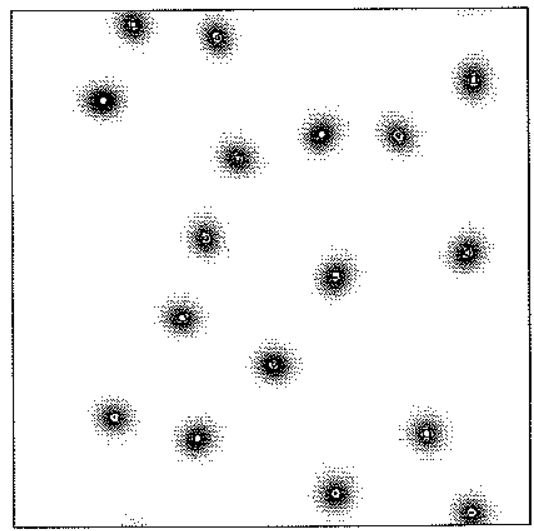

Figure 6-1: Contours of snapshots of upper layer fluctuation streamfunction for 195 degree $R_{\beta}=0.375$ experiments with wavenumber filter at 64 squared, 128 squared, and 256 squared resolution. Lower right shows nominal 128 squared hyperviscous solution for comparison. 


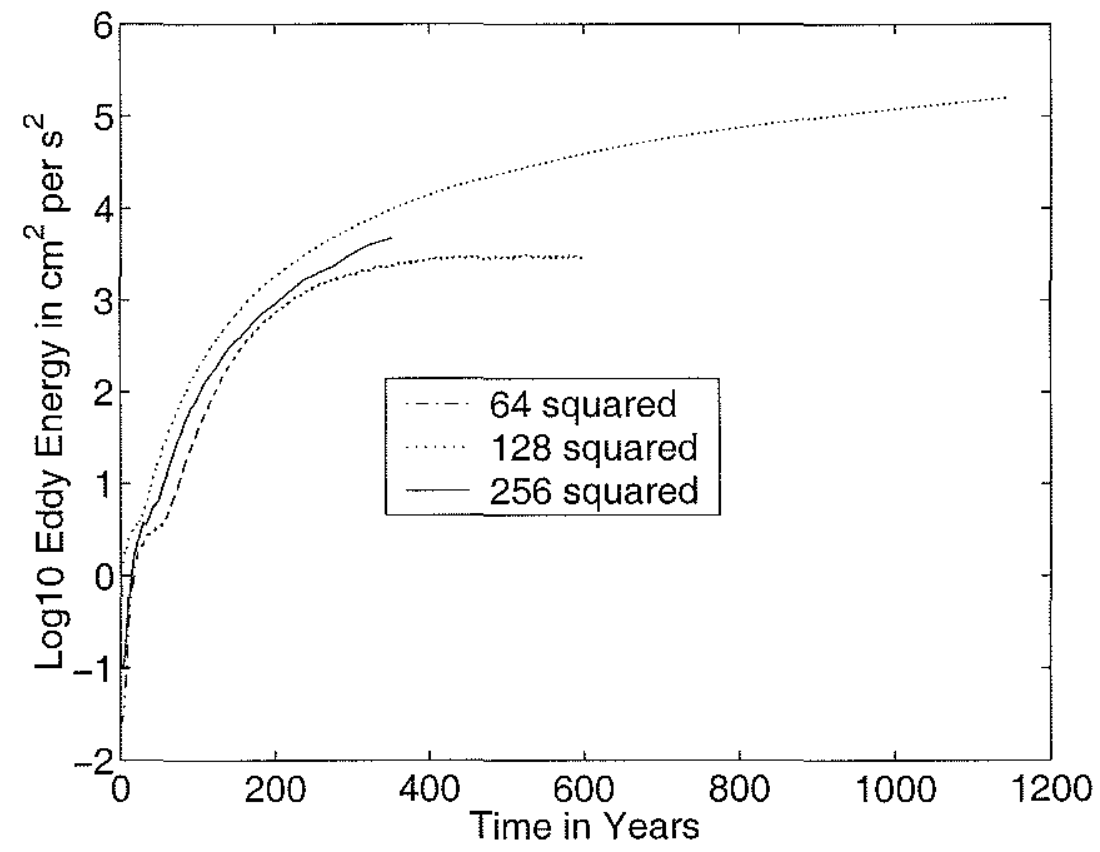

Figure 6-2: Energy versus time for 64 squared, 128 squared, and 256 squared 195 degree $R_{\beta}=0.375$ experiments with wavenumber filter.

be presented in this chapter, but they will be kept in mind.

The shear of the nominal experiment is directed 15 degrees south of west, while $R_{\beta}=0.375$. Experiments were performed with the same value of $R_{\beta}$ and magnitude of shear but with different angles (all of them south of west). Energy versus angle is plotted in figure 6-4, and snapshots of upper layer fluctuation streamfunction are contoured in figures 6-5 and 6-6. Clearly, vortices exist over a wide range of angles. Energy is not a strong function of angle over much of the range of angles studied, unlike in the strong beta solutions examined in the last chapter. Vortices exist in a westward basic state, but not in a southward basic state, under the nominal hyperviscosity. Vortices were found in a southward flow under the action of a wavenumber filter, again indicating that the vortex regime is more robust under a filter. Attempts to find vortices in flows with an eastward zonal component of shear $\left(\overline{u_{1}}-\overline{u_{2}}>0\right)$ were unsuccessful, whether hyperviscosity or wavenumber filter was used, and whether the initial condition was random or taken to be the nominal 


\section{Rbeta $=0.06$}

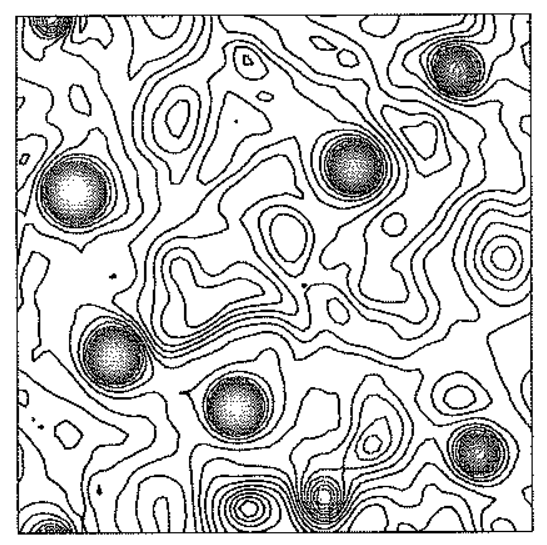

Rbeta $=0.6$

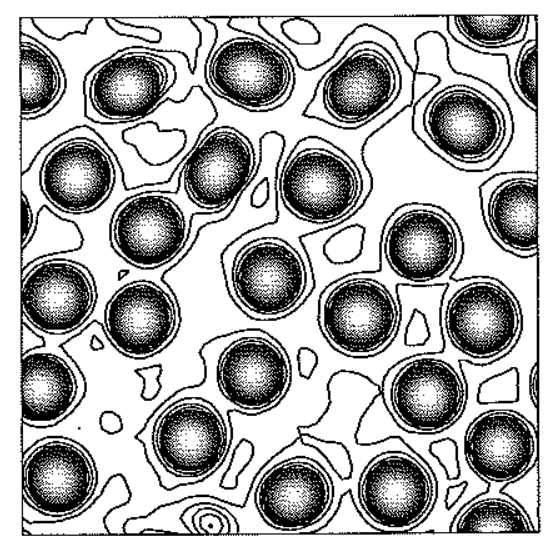

Rbeta $=6$

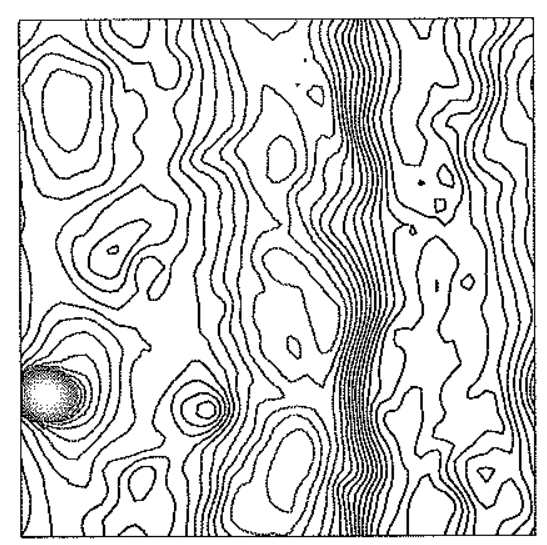

Figure 6-3: Contours of snapshots of upper layer fluctuation streamfunction in fplane zonal flow experiments having a linear slope topography varying in the $x$ direction. Resolution is 64 squared and the wavenumber filter has an exponent of 7. Bottom friction dissipation time is 580 days for the $\frac{\text { topo beta }}{\text { upper layer shear beta }}=0.06$ experiment, 290 days for the other two experiments. 
vortex solution. One of the eastward shear flow solutions is contoured in figure 6-7. The mean shear is directed 12 degrees south of east, and has a magnitude of $1.23 \mathrm{~cm}$ $\mathrm{s}^{-1}$. Beta is equal to $\frac{1}{32} \bullet 2 \bullet 10^{-11} \mathrm{~m}^{-1} \mathrm{~s}^{-1}$, the angle between layer PV gradients is 171 degrees, and $R_{\beta}=0.153$. The Ekman spindown time is 193 days. The eddy fields are isotropic $\left(\frac{u_{\text {top }}^{2}}{u_{\text {bottom }}^{2}}\right.$ is 0.96$)$, but coherent vortices are not present. Total eddy energy is $2.9 \mathrm{~cm}^{2} \mathrm{~s}^{-2}$, two orders of magnitude less than the hyperviscous vortex solutions. Since parameter space is large, we cannot be sure, in the absence of a theory for the vortices, that they do not exist in eastward shear flows, but the current evidence is that they do not.

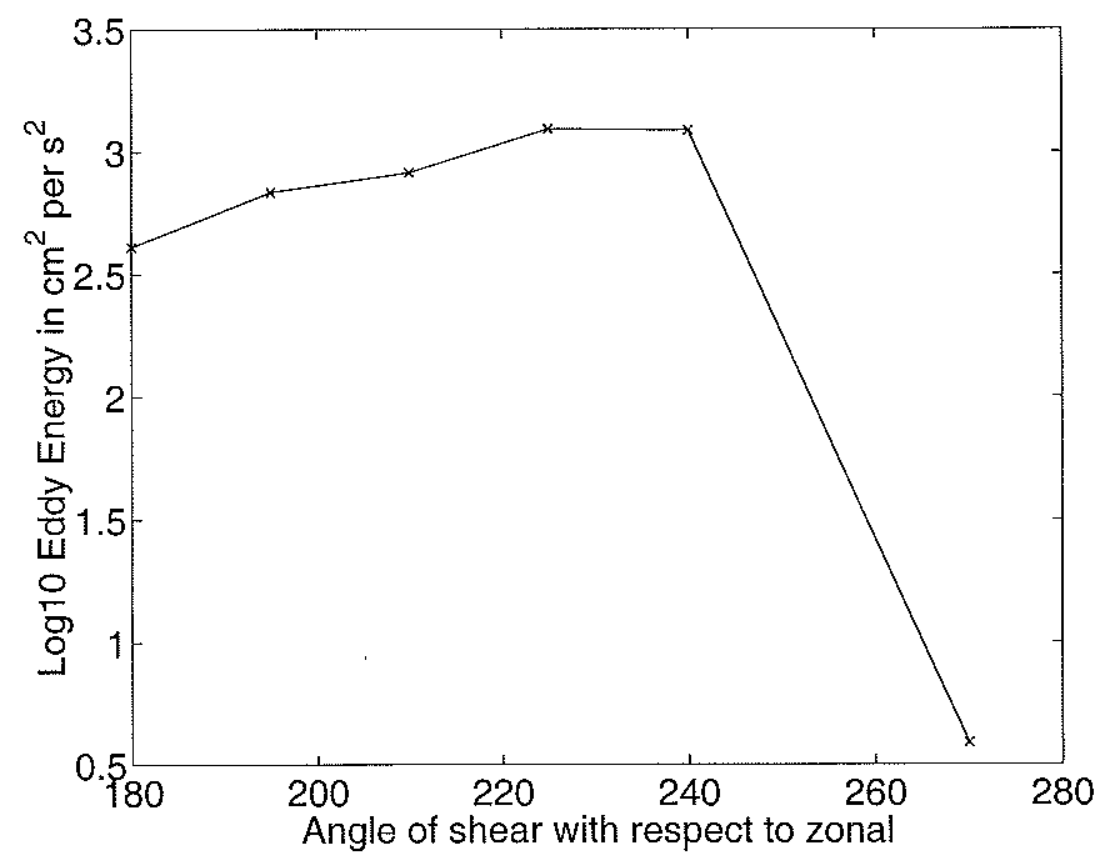

Figure 6-4: Energy versus angle for 128 squared $R_{\beta}=0.375$ experiments with hyperviscous small-scale dissipation. 
180 degrees

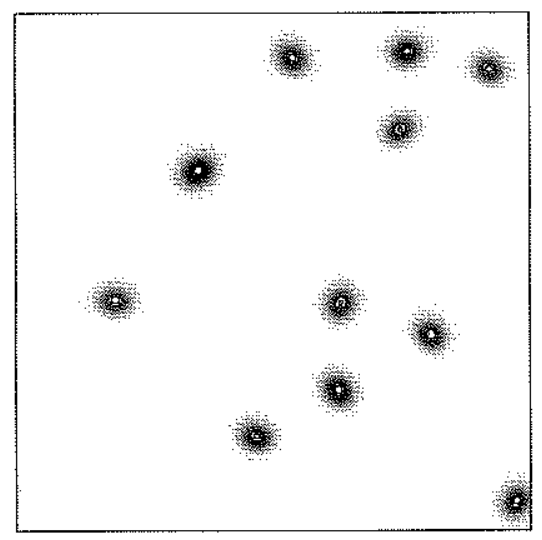

210 degrees

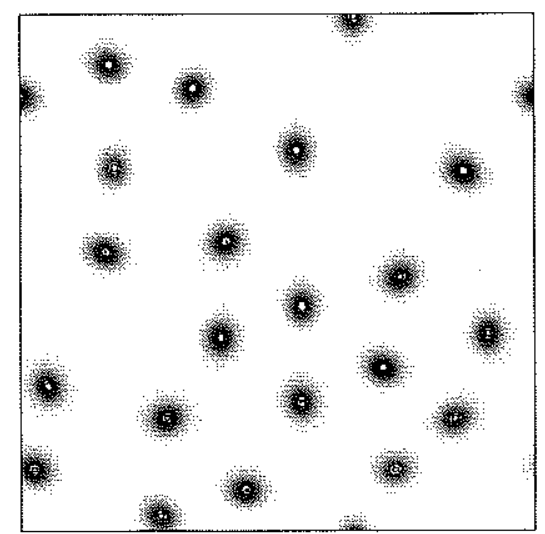

195 degrees

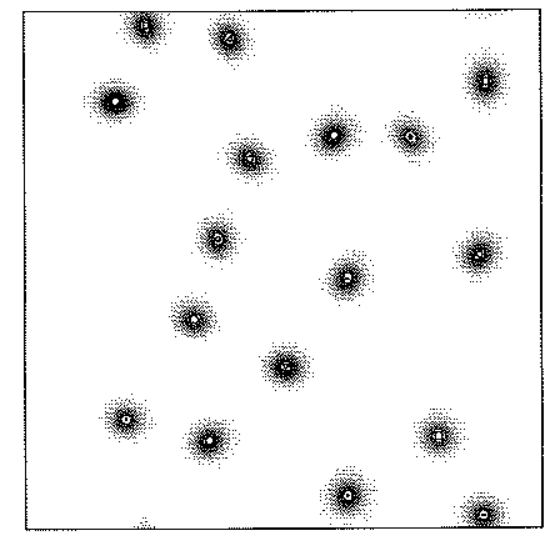

225 degrees

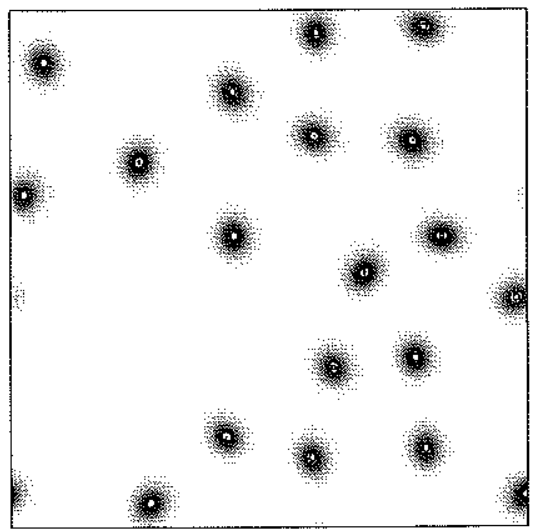

Figure 6-5: Contours of snapshots of upper layer fluctuation streamfunction for 128 squared $R_{\beta}=0.375$ experiments with hyperviscous small-scale dissipation and various angles of shear. 


\section{0 degrees}

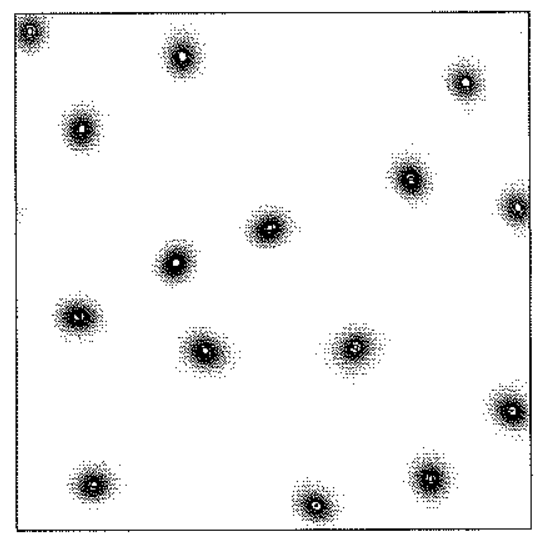

270 degrees

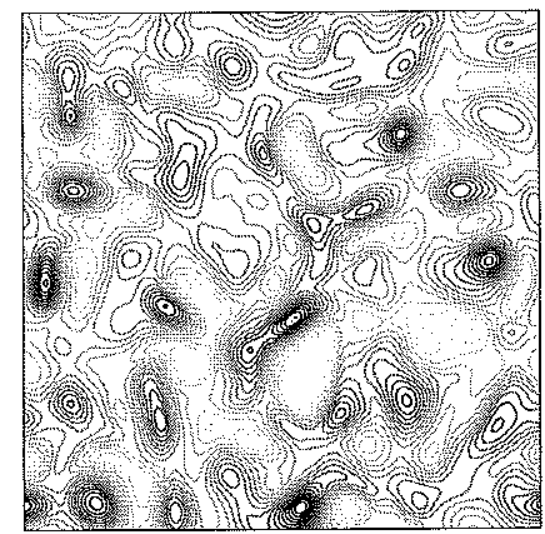

Figure 6-6: Contours of snapshots of upper layer fluctuation streamfunction for 128 squared $R_{\beta}=0.375$ experiments with hyperviscous small-scale dissipation and various angles of shear.

Ekman friction was also varied from the value used in the nominal experiment (193.4 days). Energy versus friction is plotted in figure 6-8. For comparison, energy versus friction is also plotted for the f-plane experiments of chapter 3 , and for some 64 squared wavenumber filter experiments in which friction was varied while shear angle was fixed at 45 degrees south of west. Energy of the vortex experiments is more nearly monotonic with friction than in the f-plane experiments. Modal kinetic energy ratios are plotted versus total energy and versus dissipation in figure 6-9. The most energetic vortex experiments retain more baroclinicity at a fixed energy than their f-plane counterparts. The tendency to become more barotropic with decreasing friction is clearly present, as is the fact that $\frac{H_{2}}{H_{1}}$ serves as an upper bound on $\frac{K E_{B C}}{K E_{B T}}$.

Contours of snapshots of experiments with different values of Ekman friction are displayed in figures 6-10 and 6-11. Vortices exist over a wide range of Ekman frictions, but are severely weakened for the strongest values. The monopolarity which is a signature of the vortex regime is only visible in occasional snapshots of the 24.2 day hyperviscosity experiment. (The author saw this in a MATLAB movie; not shown here.) The ratio of nonlinear to Ekman terms in the lower layer (given by 


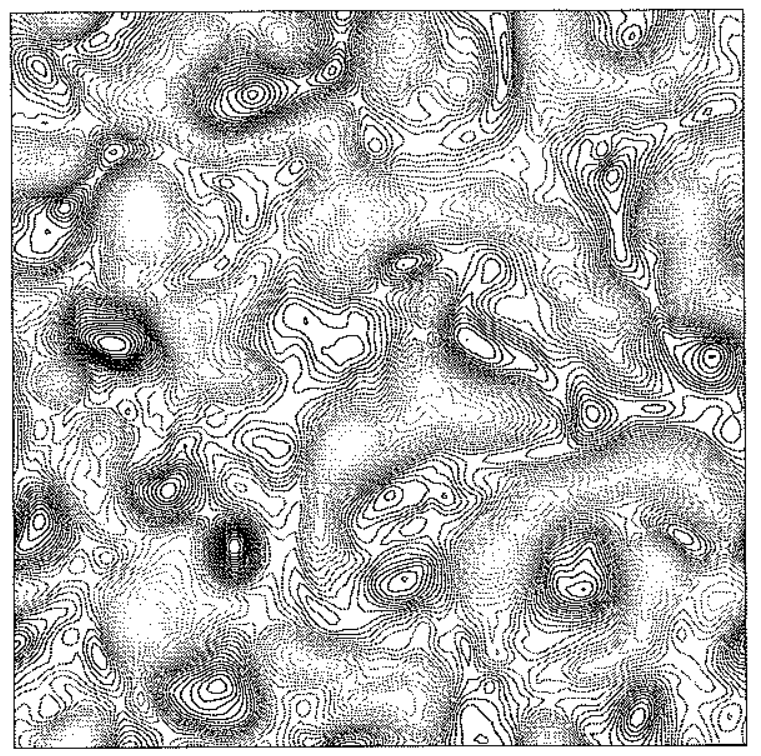

Figure 6-7: Snapshot of upper layer fluctuation streamfunction for an experiment with mean shear directed 12 degrees south of east and $R_{\beta}=0.153$. The eddy field is isotropic, but no coherent vortices are evident.

equation 3.33 ) changes from 18 to 0.5 when friction changes from 48.4 to 24.2 days in the 64 squared filter experiments. Likewise, the ratio changes from 6.5 to 0.6 for the corresponding hyperviscous experiments. Thus, as we might expect, transition from strong to weak vortices occurs when Ekman friction becomes comparable to nonlinearity. Vortex size does not change even as energy undergoes large variations. This is yet another indication that their scale is not set by the Rhines length. Barotropization with increasing energy is the only sign of the inverse cascade present in these experiments. The 48.4 day dissipation time hyperviscous experiment has a ratio of upper layer eddy to mean kinetic energy of 1366 , which is too large when compared with observations, and a baroclinicity ratio $\frac{K E_{B C}}{K E_{B T}}$ of 4.24 , also too large. Baroclinicity ratios decrease to 1.26 for the 773.8 day experiment. These baroclinicity ratios are comparable to those seen in the actual ocean, but the ratio of top layer eddy to mean kinetic energy is even more unrealistically large (29000). Thus, unlike other flat-bottom QG solutions, the vortex solutions typically retain too much baroclinic energy. 


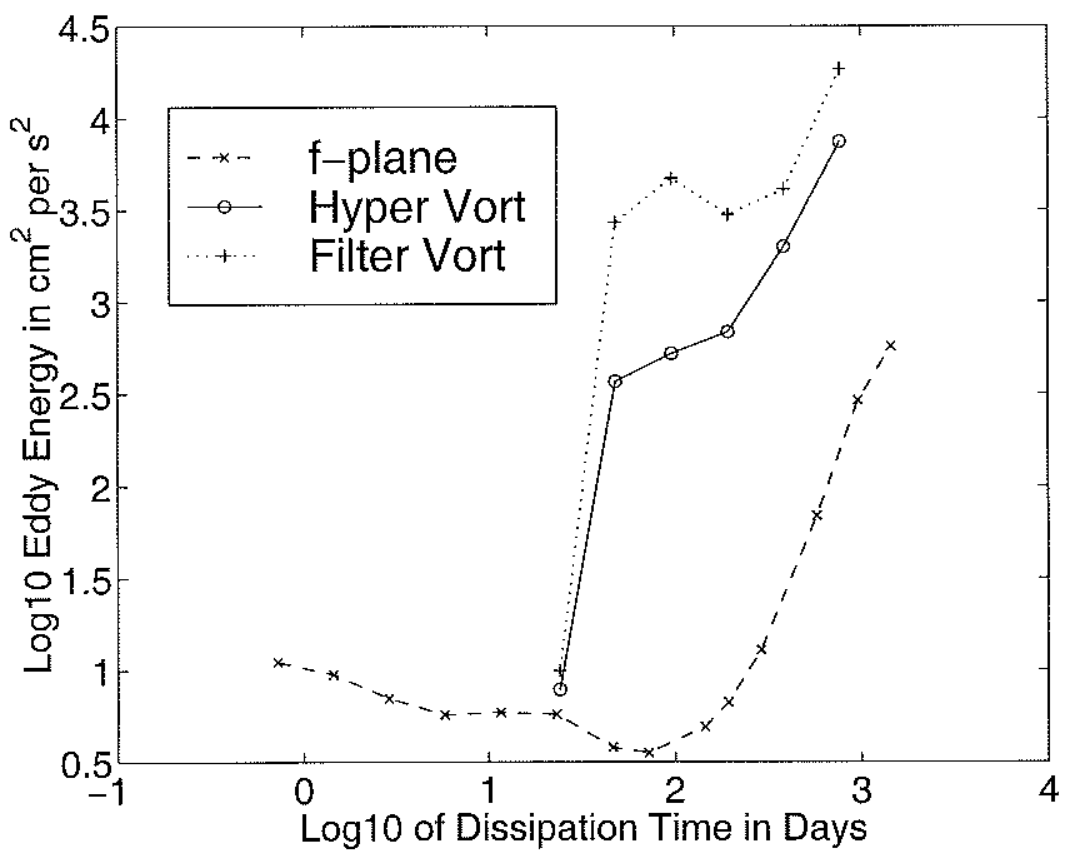

Figure 6-8: Energy versus Ekman friction for 128 squared 195 degree $R_{\beta}=0.375$ hyperviscous experiments. 64 squared 225 degree wavenumber filter experiments with the same value of $R_{\beta}$, and 256 squared f-plane experiments from chapter 3 , are included for comparison. 

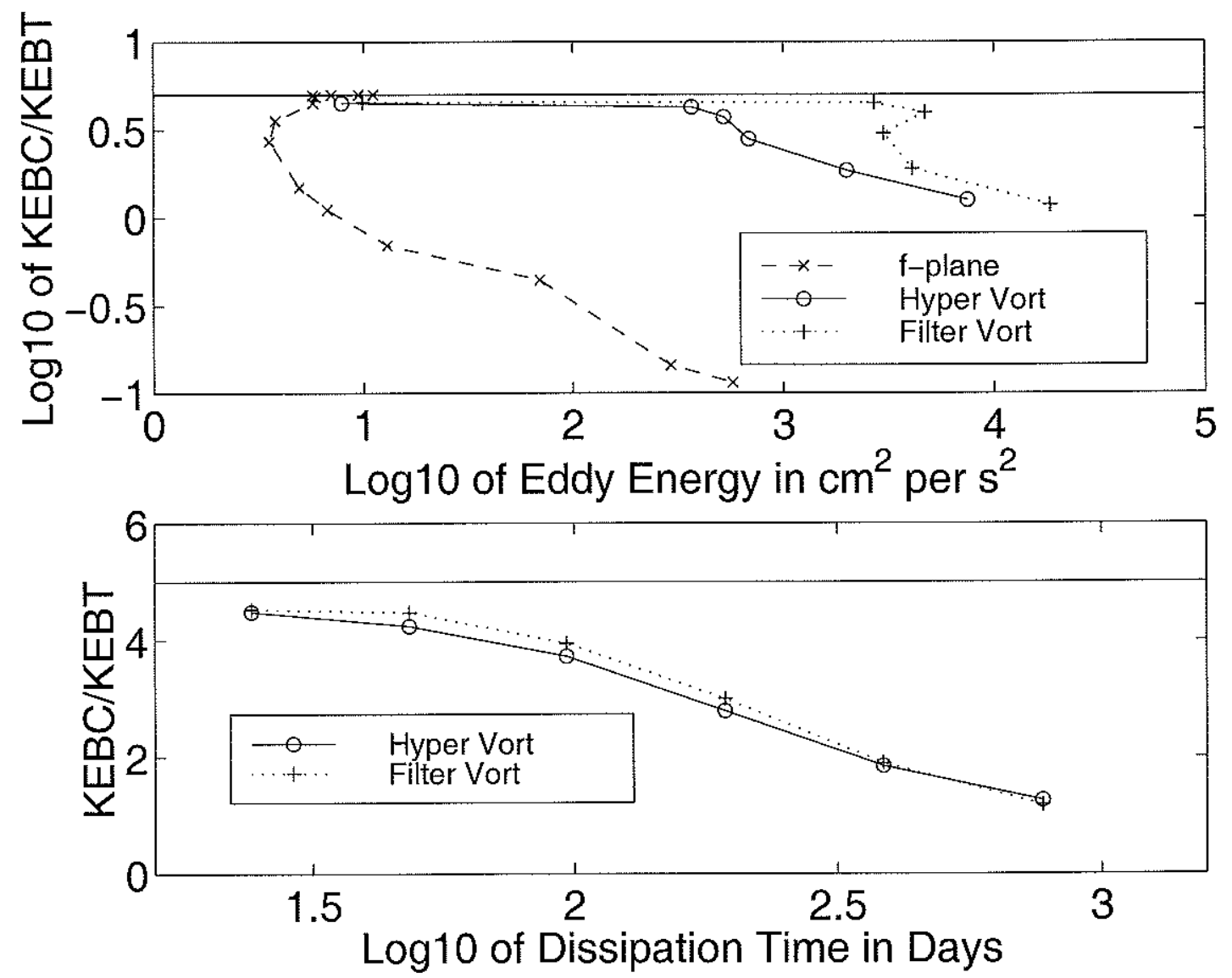

Figure 6-9: Ratio of baroclinic to barotropic kinetic energy versus total eddy energy (top panel) and versus dissipation time (bottom panel) for 128 squared 195 degree $R_{\beta}=0.375$ hyperviscous experiments. 64 squared 225 degree wavenumber filter experiments with the same value of $R_{\beta}$, and 256 squared f-plane experiments from chapter 3 , are included for comparison. Extra line indicates limiting value of $5=\frac{H_{2}}{H_{1}}$. 
773.8 days

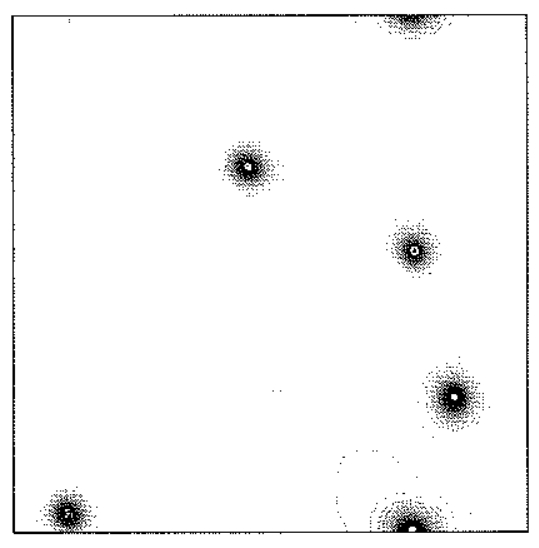

193.4 days

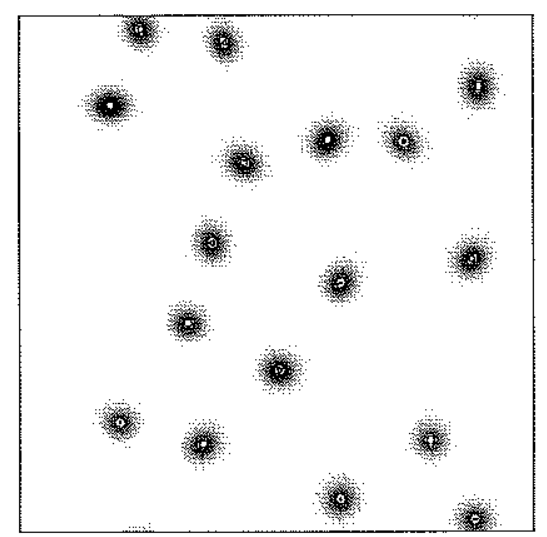

386.9 days

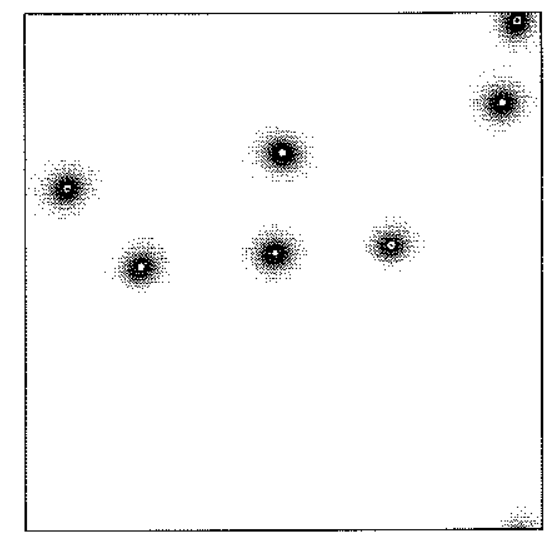

96.7 days

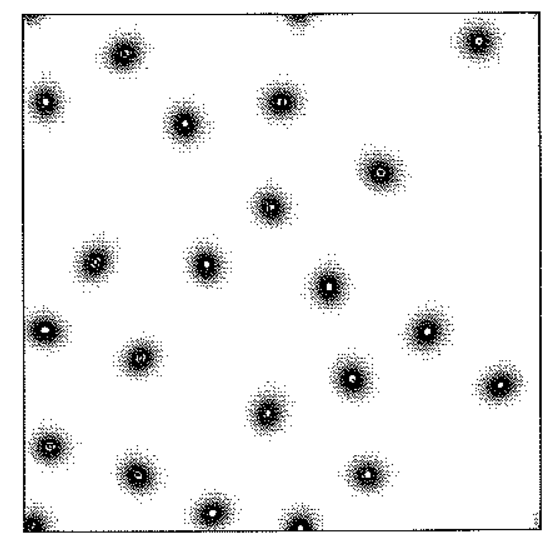

Figure 6-10: Contours of snapshots of upper layer fluctuation streamfunction for 128 squared $R_{\beta}=0.375$ experiments with hyperviscous small-scale dissipation and various values of bottom Ekman friction. 


\section{4 days}

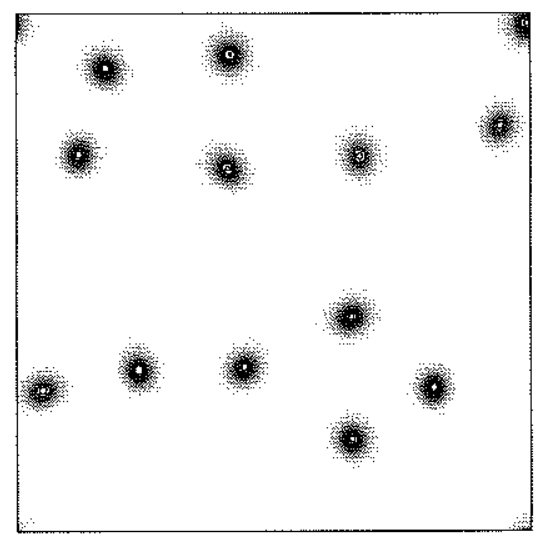

\section{2 days (filter)}

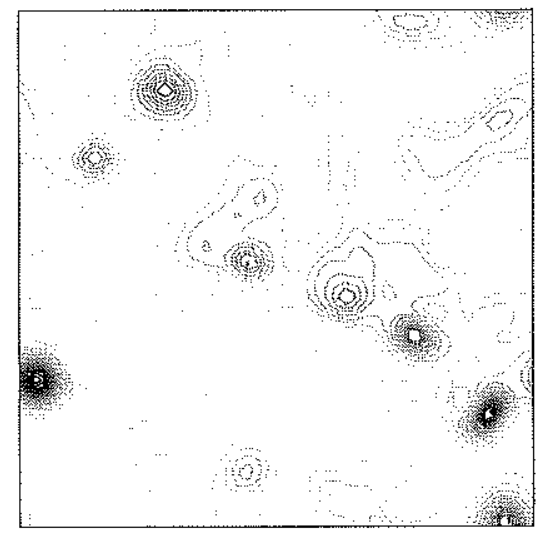

24.2 days

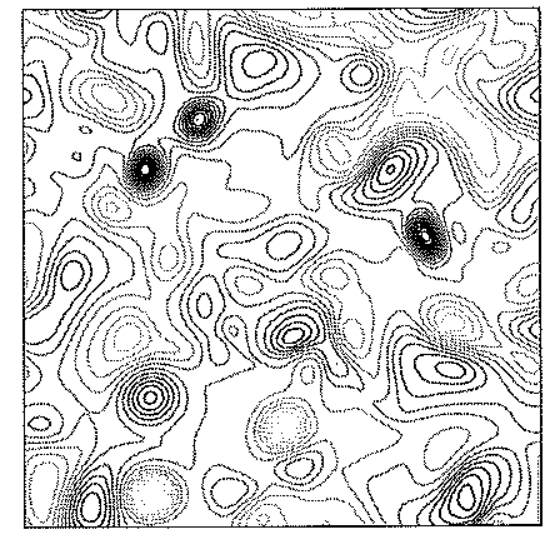

Figure 6-11: Contours of snapshots of upper layer fluctuation streamfunction for 128 squared $R_{\beta}=0.375$ experiments with hyperviscous small-scale dissipation and various values of bottom Ekman friction. The 64 squared wavenumber filter experiment sharing the largest value of Ekman friction is also shown, for comparison. 
The nominal vortex experiment has a $\delta$ value of 0.2 . Vortices do emerge in $\delta=1$ experiments (figure 6-12). The upper left panel contours an experiment with the same shear as the nominal $\delta=0.2$ experiment, with the nominal experiment serving as an initial condition. The value of $R_{\beta}$ is 0.62 , and the angle between layer PV gradients is 152 degrees. The baroclinicity ratio $\frac{K E_{B C}}{K E_{B T}}$ for this experiment is 0.41 , and the total eddy energy is $33 \mathrm{~cm}^{2} \mathrm{~s}^{-2}$. The upper right panel experiment also had the same shears, but was initialized with a small-scale random initial condition. It has fewer vortices but similar integral quantities $\left(0.42\right.$ and $\left.28 \mathrm{~cm}^{2} \mathrm{~s}^{-2}\right)$. These are to be compared with values of 2.8 and $686 \mathrm{~cm}^{2} \mathrm{~s}^{-2}$ for the nominal $\delta=0.2$ experiment. Consistent with the discussion in chapter 3 , baroclinicity ratios are bounded above by $\frac{H_{2}}{H_{1}}$. Unlike in the f-plane results, however, for a fixed value of shear, it is the $\delta=0.2$ experiments in this regime that contain more energy. The lower left panel shows an experiment in which shear is adjusted so that upper layer PV gradients are equal to those in the nominal $\delta=0.2$ experiment, with that result again serving as an initial condition. Thus $R_{\beta}$ is 0.375 , while the angle between layer PV gradients is 167 degrees. Here the energy is $616 \mathrm{~cm}^{2} \mathrm{~s}^{-2}$, more comparable to that in the nominal experiment. Thus we have more evidence that magnitudes of upper layer PV gradients play an important role in the vortex regime. Along with increased energy comes a reduced baroclinicity ratio (0.32). Finally, the lower right panel displays snapshots after equilibration of an experiment with equal upper layer potential vorticity gradients, but with a small-scale random initial condition. The baroclinicity ratio is also 0.32 but the energy is $1340 \mathrm{~cm}^{2} \mathrm{~s}^{-2}$.

A few experiments were performed with other parameters equal to those in the nominal vortex experiment, but with symmetric Ekman friction instead of bottomonly Ekman friction. These experiments were done with a wavenumber filter rather than hyperviscosity, since all indications are that vortices are more robust under the filter. However, they do not emerge from a small-scale random initial condition when symmetric friction dissipation time is 1451 days. With the same parameters, 
Same shear, ic nominal

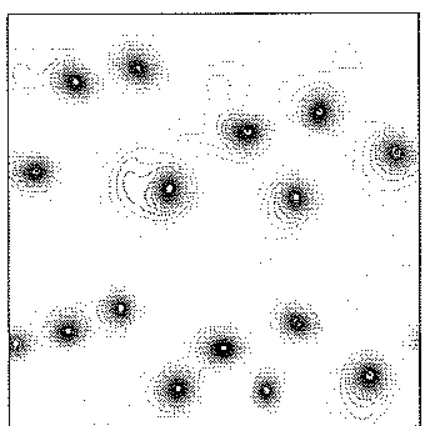

Same pvgrad, ic nominal

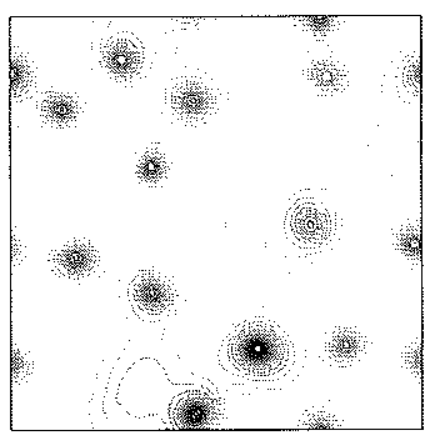

Same shear, ic random

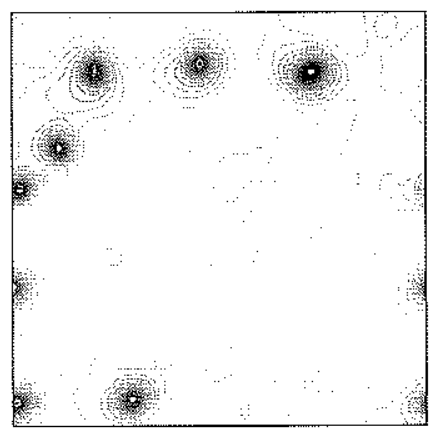

Same pvgrad, ic random

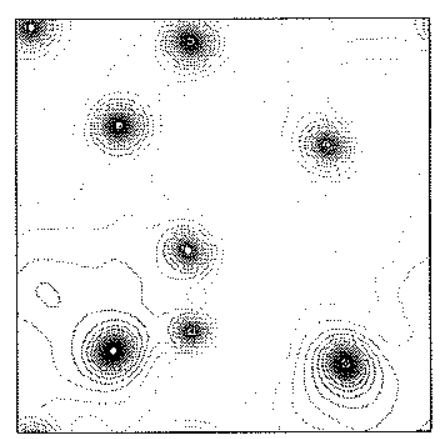

Figure 6-12: Contours of snapshots of upper layer fluctuation streamfunction for 128 squared 195 degree hyperviscous experiments having $\delta=1$ and the same value of $\beta\left(\frac{1}{16} \bullet 2 \bullet 10^{-11} \mathrm{~m}^{-1} \mathrm{~s}^{-1}\right)$ as the nominal $\delta=0.2$ vortex experiment. See text for details. 
if the initial condition is instead taken from the nominal vortex experiment, vortices quickly disappear. They also disappear when used to initialize a 387 day dissipation time symmetric friction experiment. Apparently, the vortices only emerge in a layer which has no Ekman friction. That they do reside in the upper layer is made clear in figure 6-13, which shows lower layer fluctuation streamfunction for the nominal experiment. Vortices are not visually evident, nor are they in the lower layer of any of the forced-dissipated experiments performed in this dissertation. Many of the interesting features we have described in this thesis-the fronts in the highly viscous regime of chapter 3 , the jets of chapter 5 , and the vortices of this chapter-exist in the upper layer of our forced-dissipated experiments. In that layer there is no Ekman friction, and Reynolds numbers can become large. Most of the jets and vortices that exist in the actual ocean also exist in surface layers, where friction is weak.

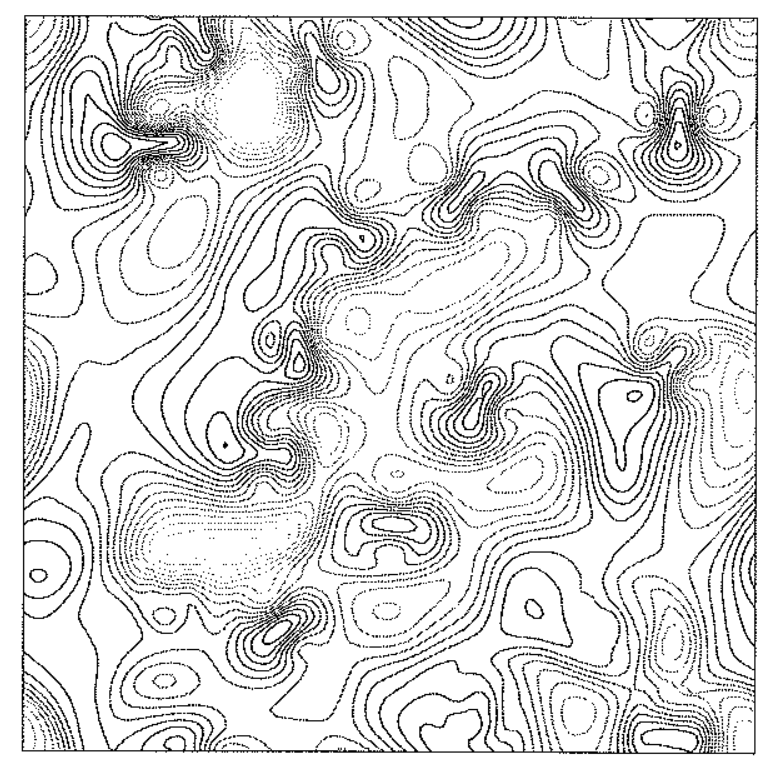

Figure 6-13: Contours of snapshot of lower layer fluctuation streamfunction for nominal 128 squared hyperviscous vortex experiment. Vortices are not evident. 


\subsection{Cyclone-anticyclone asymmetry and depen- dence on initial conditions}

Thus far we have seen two examples of symmetry breaking in the vortex regime. One is that eastward flows, unlike westward flows, do not seem to generate vortex solutions. The other is that vortex fields are monopolar. Let us now discuss the symmetry properties of the governing equations. We use the lower layer PV equation (equation 2.2) for purposes of illustration. It is:

$$
\begin{gathered}
\frac{\partial q_{2}}{\partial t}+\overline{u_{2}} \frac{\partial q_{2}}{\partial x}+ \\
+\overline{v_{2}} \frac{\partial q_{2}}{\partial y}-\frac{\delta\left(\overline{v_{1}}-\overline{v_{2}}\right)}{(1+\delta) R_{d}^{2}} \frac{\partial \psi_{2}}{\partial y}+\left(\beta+\frac{\delta\left(\overline{u_{2}}-\overline{u_{1}}\right)}{(1+\delta) R_{d}^{2}}\right) \frac{\partial \psi_{2}}{\partial x} \\
+J\left(\psi_{2}, q_{2}\right)=-R_{2} \nabla^{2} \psi_{2}+\nu \nabla^{6} q_{2}
\end{gathered}
$$

when hyperviscosity is the small-scale dissipation. We first discuss what happens when $\overline{u_{1}}$ and $\overline{u_{2}}$ are negated. For simplicity in this case, suppose $\delta=1$. Then $\frac{\partial \overline{q_{2}}}{\partial y}=$ $\beta+\frac{\overline{u_{2}}-\overline{u_{1}}}{(1+\delta) R_{d}^{2}}$. Negating $\overline{u_{1}}$ and $\overline{u_{2}}$ means that the shear part of $\frac{\partial \overline{q_{2}}}{\partial y}$ flips sign. However, $\beta$ does not. Therefore 6.1 is not invariant to a change in sign of the zonal velocities, which is consistent with the fact that vortices exist in flows with a westward zonal component of shear but apparently not in flows with an eastward zonal component of shear. If Ekman friction were vertically symmetric, then in the $\delta=1$ case the upper and lower layer subscripts could be switched and the layer PV equations 2.1 and 2.2 would be invariant. Then there could be no asymmetry between eastward and westward shear flows. We have seen, however, that the vortex regime does not exist with symmetric friction. It exists with bottom Ekman friction, under which it is impossible to switch the layer indices. Therefore the asymmetry between eastward and westward flows in the vortex regime is connected to the vertical asymmetry of the Ekman friction.

Now let us examine the symmetry properties of cyclones and anticyclones. The nominal solution is a field of cyclones, as are all other vortex solutions examined 
thus far. That the vortices are all of the same sign is at this point still unexplained. For now, let us accept that it is so, and ask whether the negative of an equilibrated solution is also a solution. Negating $\psi_{2}$ (and $\psi_{1}$ ) implies that $q_{2}$ is also negated (see equation 2.7). Therefore $\frac{\partial q_{2}}{\partial t}$ changes sign, as do all other terms in the equation (which are linear in either $\psi_{2}$ or $q_{2}$ ) save the Jacobian (which is nonlinear since both $\psi_{2}$ and $q_{2}$ are present). If the nonlinear term were absent, the governing equations would be invariant to the transformation $\psi_{2} \rightarrow-\psi_{2}$. The presence of the Jacobian complicates matters. Since its calculation involves taking derivatives, the Jacobian term could be negated by negating $\psi_{2}$ and one (but not both) of the spatial coordinates. If planetary beta and mean shear terms were absent, either $x$ or $y$ could be negated along with $\psi_{2}$, and 6.1 would be invariant. Since either $\beta$ or a mean shear flow impose a direction, the presence of either limits the choice of spatial coordinates to flip. Supposing $\overline{v_{1}}$ and $\overline{v_{2}}$ were zero, then 6.1 would be invariant under $\psi_{2} \rightarrow-\psi_{2}, y \rightarrow-y$, but not under $\psi_{2} \rightarrow-\psi_{2}, x \rightarrow-x$. McWilliams and Flierl (1979), and probably others, were aware of the invariance of freely evolving QG on a beta plane under the transformations $\psi_{2} \rightarrow-\psi_{2}, y \rightarrow-y$. A zonal shear can also be added, and 6.1 will be invariant under this transformation. However, the most general case of 6.1-a nonzonal flow on a beta plane-is not invariant under this transformation. Symmetry between cyclones and anticyclones is broken when the nonlinear term, which requires one of the spatial coordinates to be inverted to maintain invariance, is present concurrent with a nonzonal flow on a beta plane, which implies that the directions imposed by $\beta$ and shear will be different. This makes it impossible to find a coordinate to invert which will make 6.1 invariant. We will see concrete consequences of this symmetry breaking shortly.

Equation 6.1 is invariant under the transformations $\psi_{2} \rightarrow-\psi_{2}, y \rightarrow-y, \overline{v_{1}} \rightarrow$ $-\overline{v_{1}}$, and $\overline{v_{2}} \rightarrow-\overline{v_{2}}$. This was tested by switching the nominal solution $\psi(x, y)$ to $-\psi(x,-y)$ in both layers, and negating the mean meridional flow, but otherwise keeping the parameters the same. The number of vortices (now anticyclones) stayed 
constant, as did the equilibrated energy (in a statistical sense, that is). Experiments also confirmed that a vortex solution formed in a westward zonal flow is invariant under $\psi \rightarrow-\psi, y \rightarrow-y$. On the other hand, the equations predict that a solution developed in a nonzonal flow is not invariant under $\psi \rightarrow-\psi, y \rightarrow-y$ if $\bar{v}$ is kept fixed. Figure 6-14 plots energy versus time for an experiment in which the inverted nominal experiment was used to initialize the nominal mean flow. Energy reduces by about an order of magnitude. This, then, is how the asymmetry manifests itself. The number of vortices has reduced by one (figure 6-15). Figures 6-16 and 6-17 reveal that the vortices stay anticyclonic but undergo a reduction of amplitude. Thus there are two related cyclone-anticyclone asymmetries present in the vortex regime. One is that, for certain values of $R_{\beta}$, a monopolar vortex field emerges. The other is that, if the mean shear is southwestward (northwestward), energy levels are higher (lower) for a field of cyclones than for a field of anticyclones.

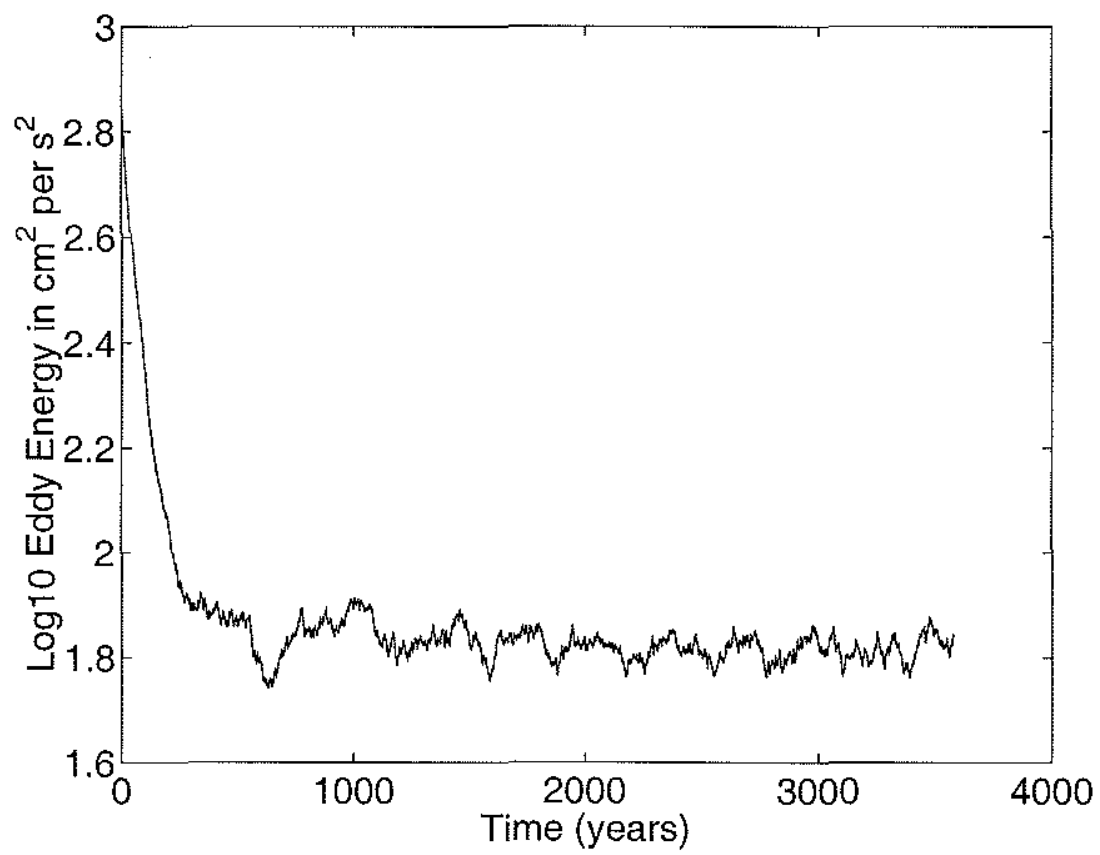

Figure 6-14: Energy versus time for the 128 squared hyperviscous experiment in which an inverted nominal solution is used to initialize the nominal mean flow.

We have seen that negating the nominal solution of cyclones leads to a lower 
Initial

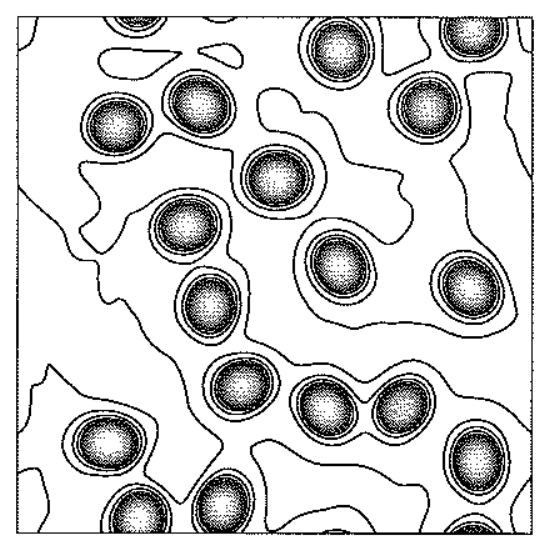

Final

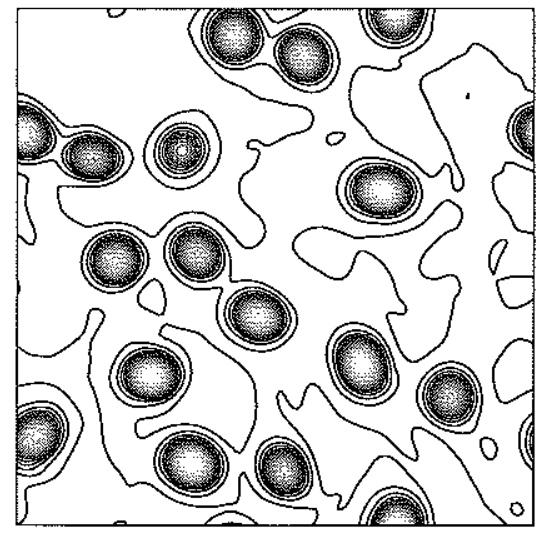

Figure 6-15: Initial and final upper layer fluctuation streamfunction for the 128 squared hyperviscous experiment in which an inverted nominal solution is used to initialize the nominal mean flow.

energy state of anticyclones. We have also seen signs that vortex solutions emergent in a particular experiment depend on initial condition. Most experiments to date have been initialized by a field that is randomly generated in space (c.f. top left panel in figure 5-38). This initial condition has always led to a high-energy state of cyclones, when the mean flow is southwestward. We now initialize the nominal parameters with a variety of fields, to determine how powerful the cyclone and anticyclone attractors are. Initial conditions used include spectra of various powers, and equilibrated solutions taken from other parts of the thesis. Figures 6-18 through 621 display experiments that resulted in cyclones. Those resulting in anticyclones are shown in figures 6-22 and 6-23. Not shown are experiments initialized by twice the nominal streamfunction, by half the nominal streamfunction, and by the negative of the small-scale random initial condition. The first two of these returned to the nominal energy levels and stayed cyclonic, while the latter produced cyclones of slightly higher energy. Energy levels and numbers of vortices vary between experiments. However, all initial conditions produced either a high energy state of cyclones, or a low energy state of anticyclones. Note that anticyclones were not produced by 


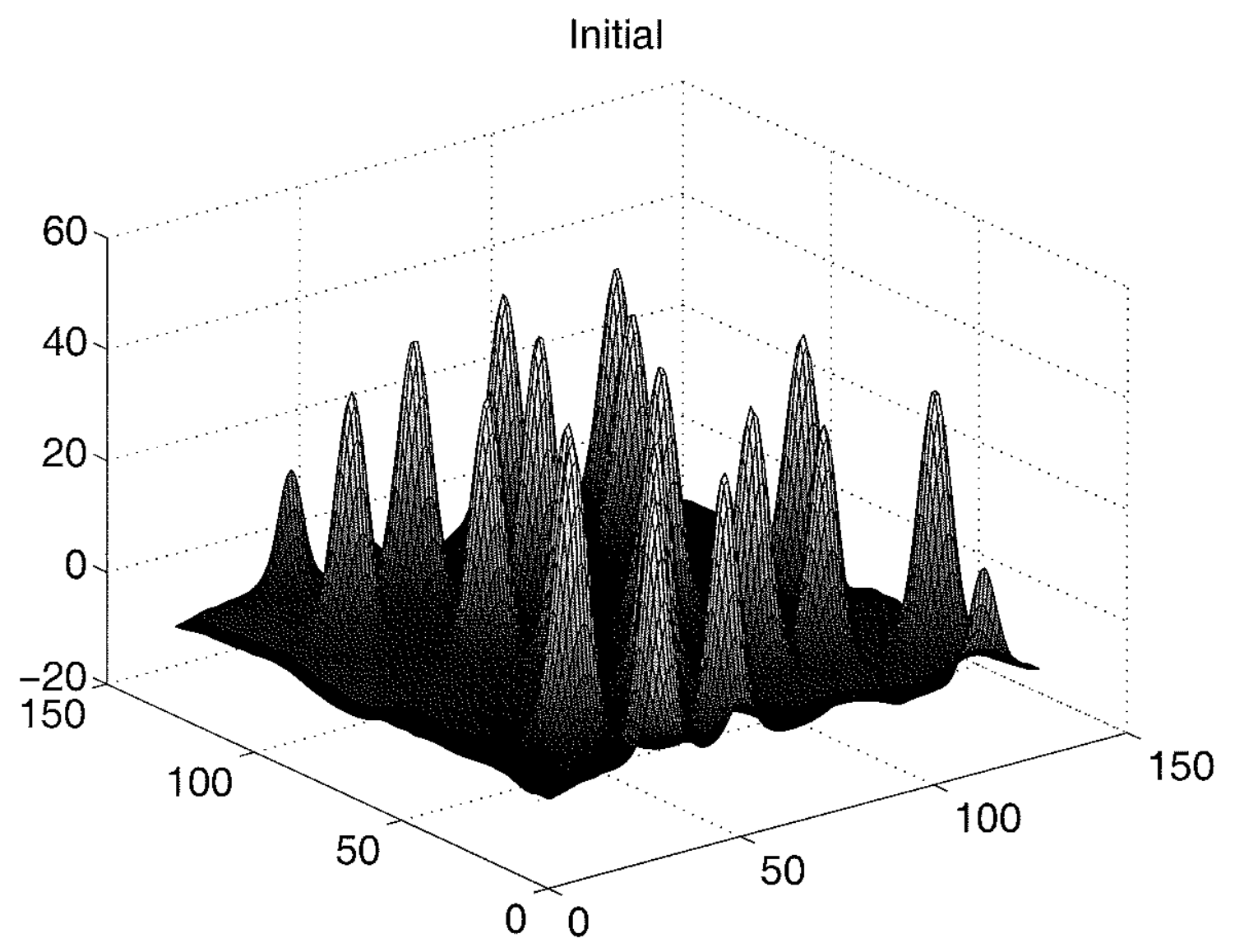

Figure 6-16: Initial upper layer fluctuation streamfunction for the 128 squared hyperviscous experiment in which an inverted nominal solution is used to initialize the nominal mean flow. 


\section{Final}

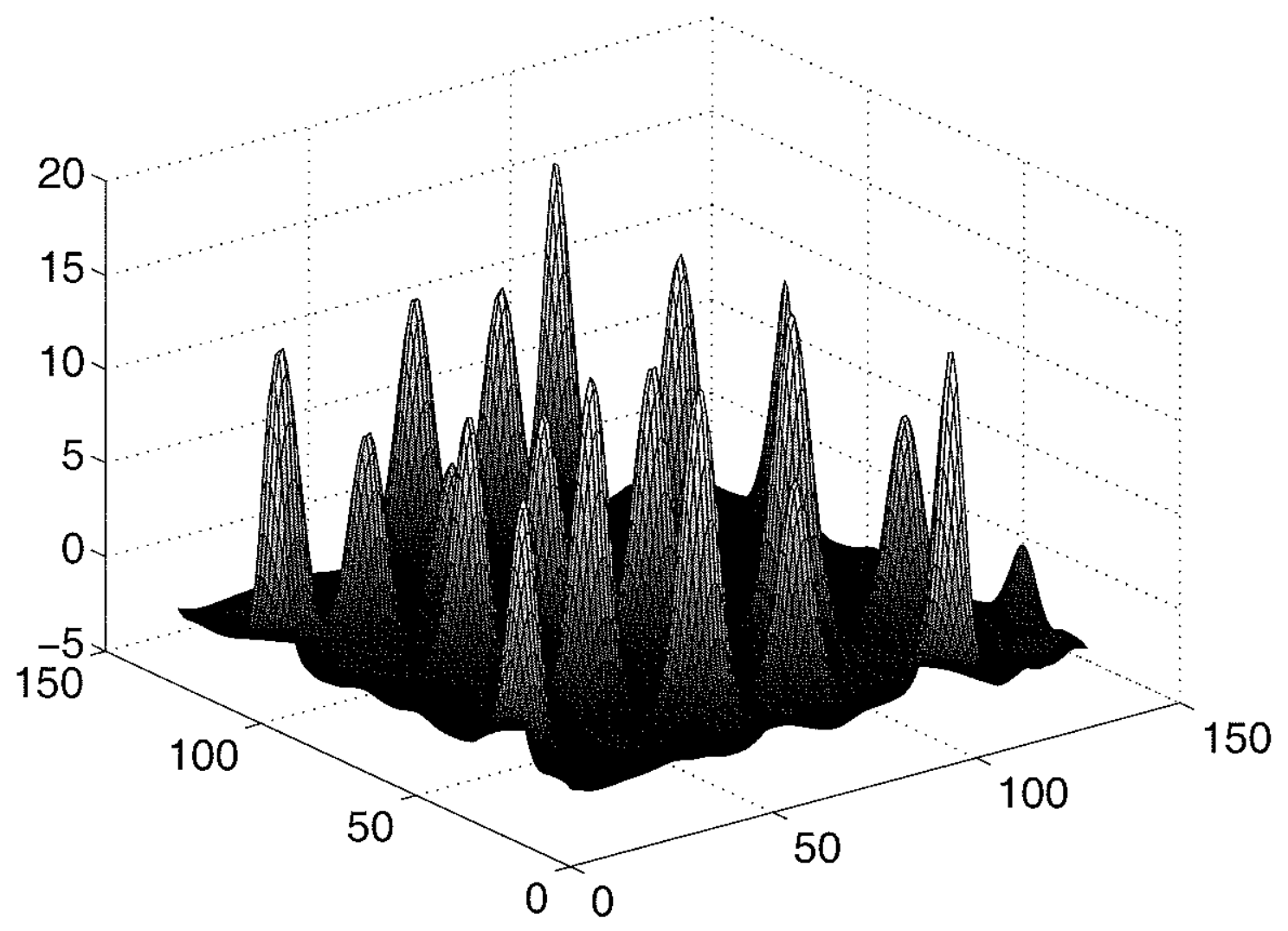

Figure 6-17: Final upper layer fluctuation streamfunction for the 128 squared hyperviscous experiment in which an inverted nominal solution is used to initialize the nominal mean flow. 
any of the random initial conditions attempted. The baroclinicity ratio $\frac{K E_{B C}}{K E_{B T}}$ was in the range 2.6 to 2.8 for all of these experiments. Although vortex number varies from experiment to experiment, it is an observation of this author, formed through observing time evolutions of many experiments, that once equilibration is reached within a particular experiment, the number of vortices remains constant. Despite the forcing and dissipation present, there is apparently no creation of new vortices or destruction of old ones, once equilibration is reached. 
Top I.C.

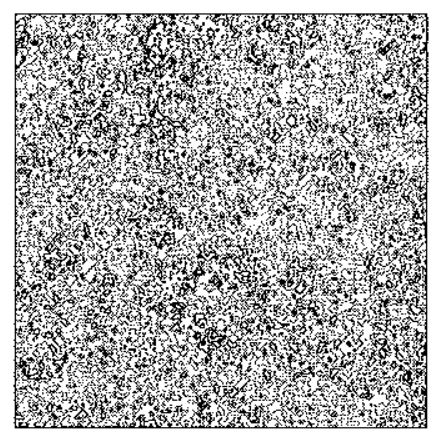

Top Final

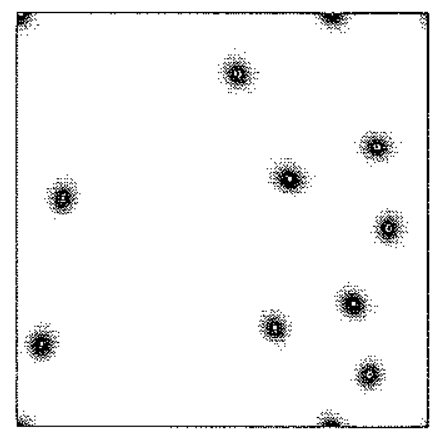

Bottom I.C.

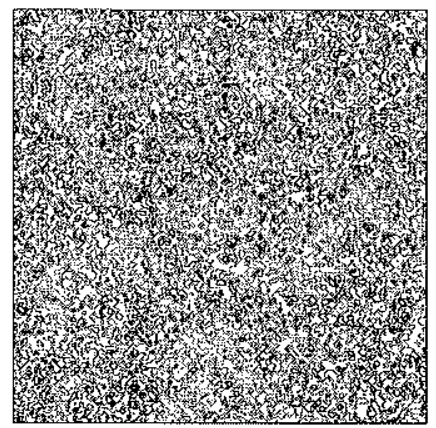

Top Final

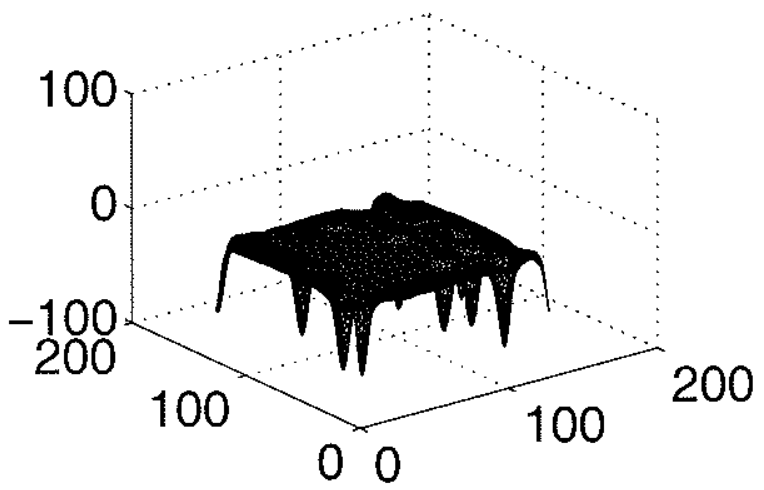

Figure 6-18: Fluctuation streamfunction contours and surface plot for snapshot of cyclones resulting from 128 squared hyperviscous experiment initialized with $\mathrm{k}^{-1}$ spectrum. Total eddy energy is $948 \mathrm{~cm}^{2} \mathrm{~s}^{-2}$. Note that the nominal solution consists of 17 cyclones and a total eddy energy of $686 \mathrm{~cm}^{2} \mathrm{~s}^{-2}$. 
Top I.C.

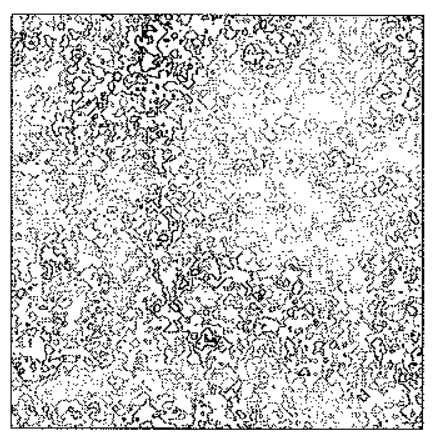

Top Final

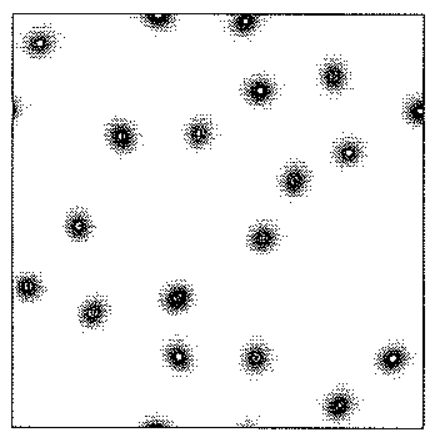

Bottom I.C.

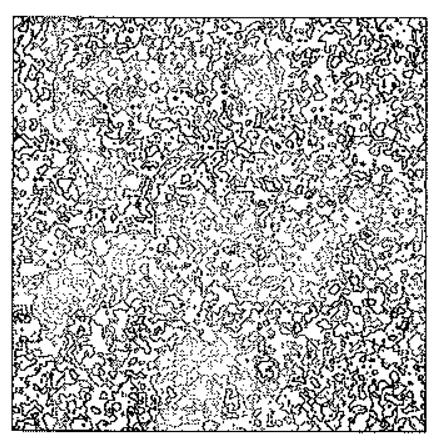

Top Final

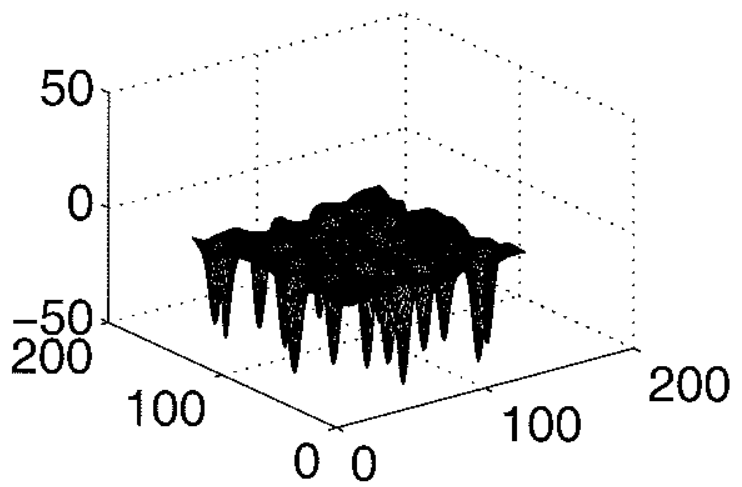

Figure 6-19: Fluctuation streamfunction contours and surface plot for snapshot of cyclones resulting from 128 squared hyperviscous experiment initialized with $\mathrm{k}^{-2}$ spectrum. Total eddy energy is $612 \mathrm{~cm}^{2} \mathrm{~s}^{-2}$. 
Top I.C.

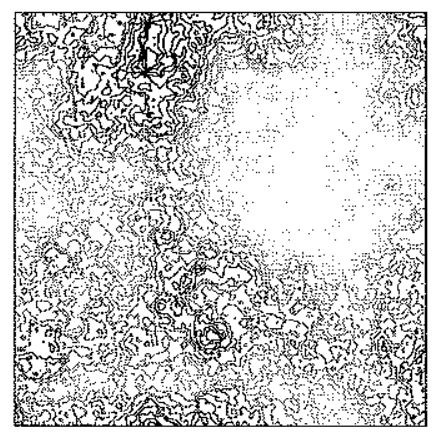

Top Final

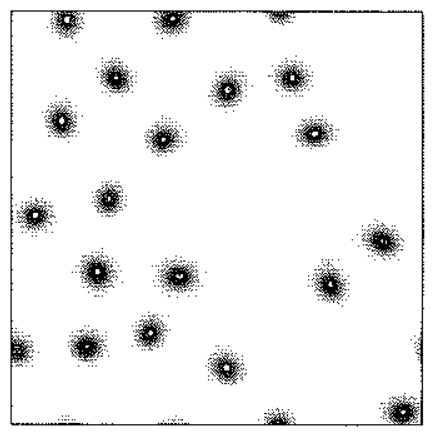

Bottom I.C.

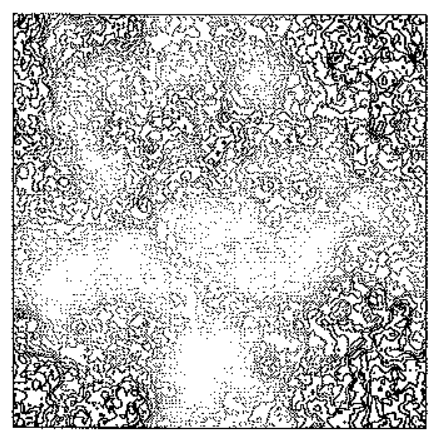

Top Final

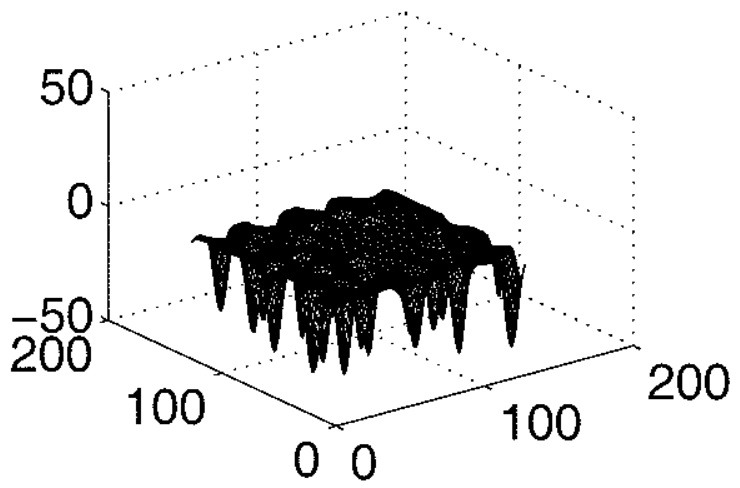

Figure 6-20: Fluctuation streamfunction contours and surface plot for snapshot of cyclones resulting from 128 squared hyperviscous experiment initialized with $\mathrm{k}^{-3}$ spectrum. Total eddy energy is $555 \mathrm{~cm}^{2} \mathrm{~s}^{-2}$. 
Top I.C.

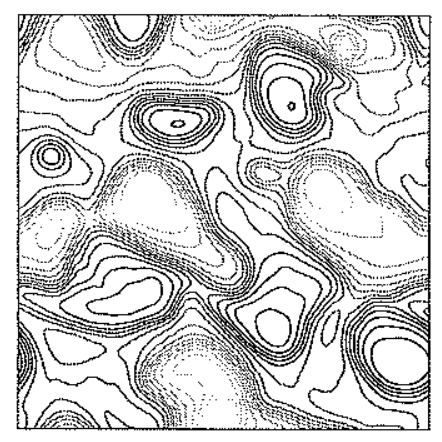

Top Final

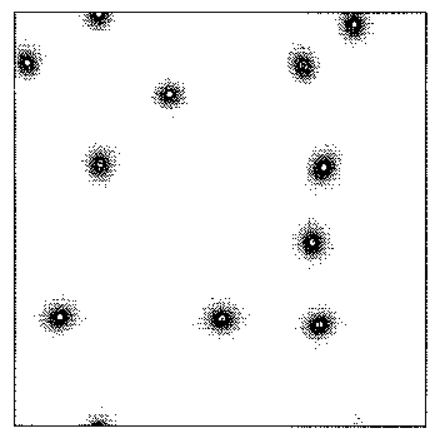

Bottom I.C.

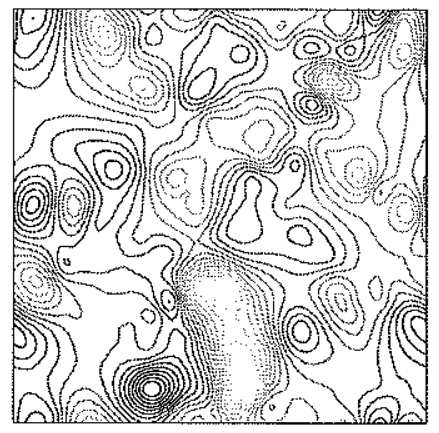

Top Final

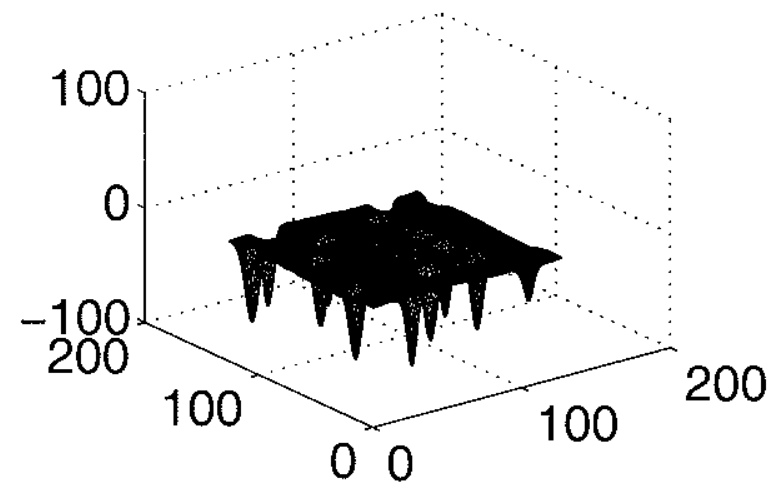

Figure 6-21: Fluctuation streamfunction contours and surface plot for snapshot of cyclones resulting from 128 squared hyperviscous experiment initialized with $\delta=1$, f-plane 2.90 day dissipation time experiment. Total eddy energy is $956 \mathrm{~cm}^{2} \mathrm{~s}^{-2}$. 
Top I.C.

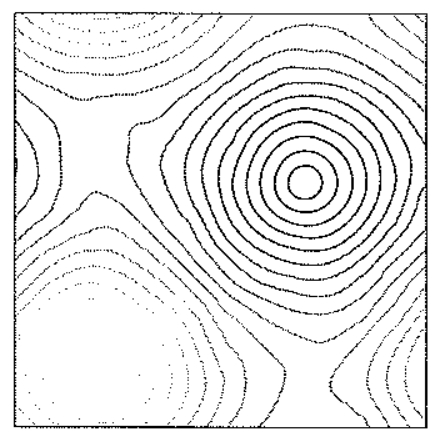

Top Final

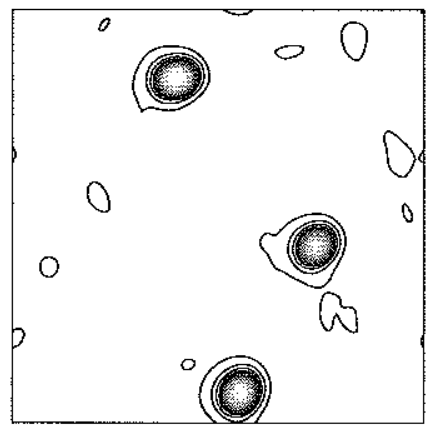

Bottom I.C.

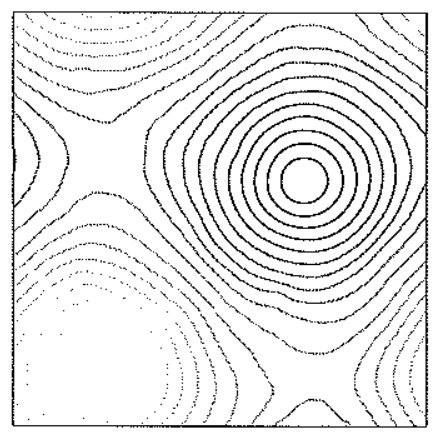

Top Final

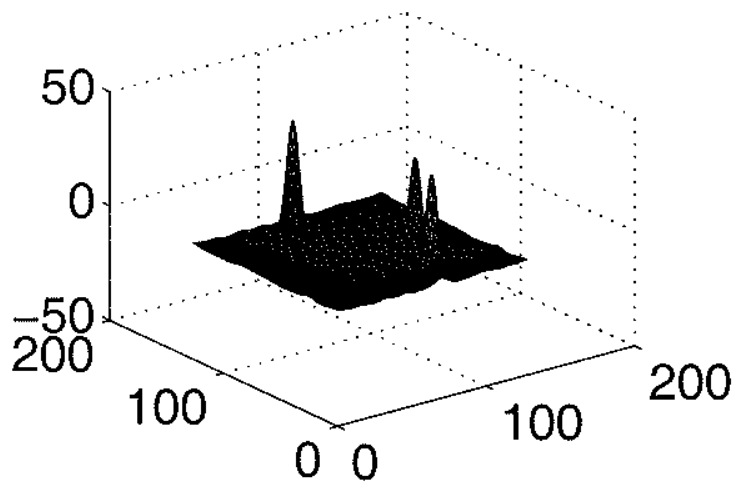

Figure 6-22: Fluctation streamfunction contours and surface plot for snapshot of anticyclones resulting from 128 squared hyperviscous experiment initialized with $\delta=1$, f-plane 1451 day dissipation time experiment. Total eddy energy is $144 \mathrm{~cm}^{2}$ $\mathrm{s}^{-2}$. Note that the energy of the anticyclones in $6-17$ is $66 \mathrm{~cm}^{2} \mathrm{~s}^{-2}$. 
Top I.C.

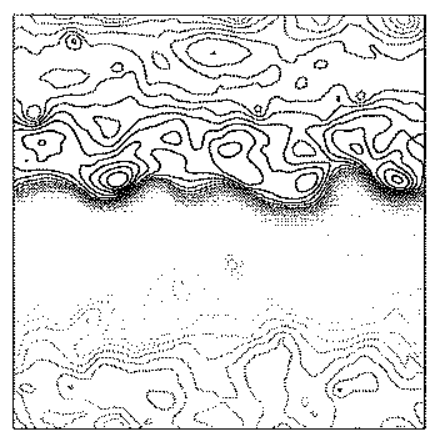

Top Final

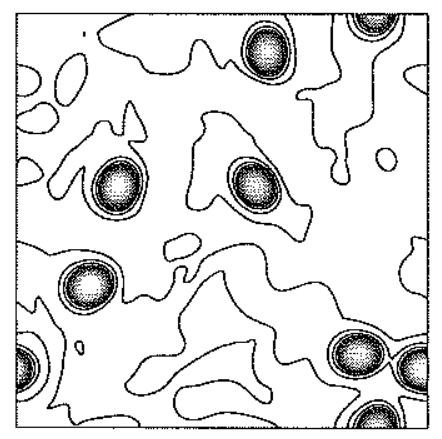

Bottom I.C.

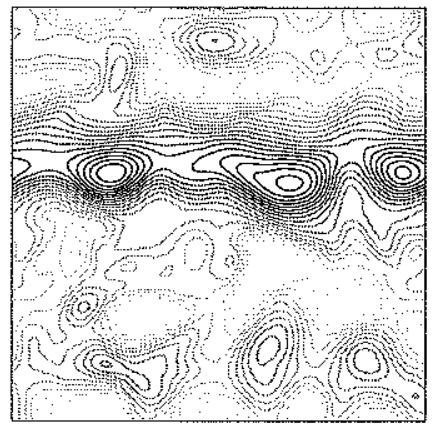

Top Final

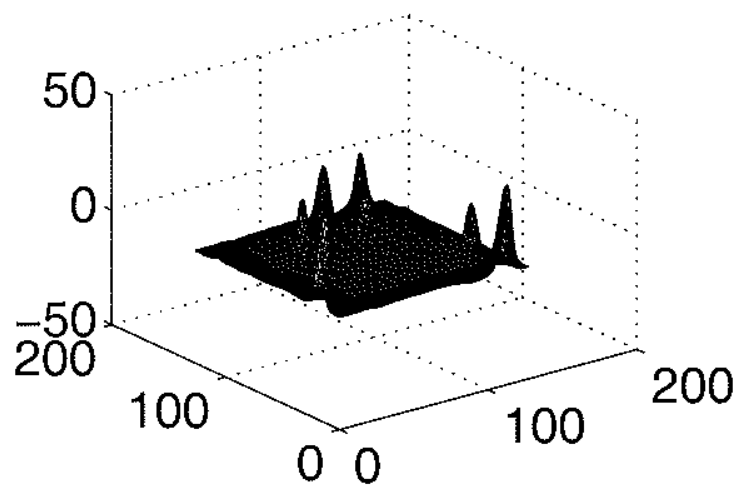

Figure 6-23: Fluctuation streamfunction contours and surface plot for snapshot of anticyclones resulting from 128 squared hyperviscous experiment initialized with 225 degree experiment from section 5.2. Total eddy energy is $118 \mathrm{~cm}^{2} \mathrm{~s}^{-2}$. 


\subsection{Spin-down and freely decaying experiments initialized by vortex solutions}

In this section the nominal vortex solution is used to initialize three types of experiments: 1) equivalent barotropic freely decaying experiments, 2) two-layer freely decaying experiments, and 3) two-layer experiments with Ekman friction in the bottom layer. These experiments are designed to examine vortex behavior in the absence of forcing, or of Ekman dissipation, or of both. The results are complicated, but as a whole they enhance our understanding of the vortex regime. They were all run with hyperviscosity as a small-scale dissipation. To aid understanding of the one-layer results we provide a brief theoretical digression on cascades in the equivalent barotropic system, often called the " 1.5 layer" model.

\subsubsection{Freely evolving equivalent barotropic turbulence}

When $R_{d}$ is introduced into a freely evolving one-layer quasi-geostrophic system, the governing equation is:

$$
\frac{\partial}{\partial t}\left(\nabla^{2} \psi-\frac{1}{R_{d}^{2}} \psi\right)+J\left(\psi, \nabla^{2} \psi\right)=0
$$

where as usual we ignore small-scale dissipation. The energy equation is obtained via multiplication by $\psi$ and integration:

$$
\frac{\partial}{\partial t} \iint \frac{1}{2}\left[(\nabla \psi)^{2}+\frac{1}{R_{d}^{2}} \psi^{2}\right] d x d y=\frac{\partial}{\partial t} \int E(k) d k=0
$$

where the energy spectrum $E(k)$ now contains potential as well as kinetic energy. At this point, instead of multiplying by total $q=\nabla^{2} \psi-\frac{1}{R_{d}^{2}} \psi$, as would be standard procedure, the author decided to multiply by $\nabla^{2} \psi$, and then integrate to obtain: 


$$
\frac{\partial}{\partial t} \iint \frac{1}{2}\left[\left(\nabla^{2} \psi\right)^{2}+\frac{1}{R_{d}^{2}}(\nabla \psi)^{2}\right] d x d y=\frac{\partial}{\partial t} \int k^{2} E(k) d k=0
$$

The same argument for a cascade in purely two-dimensional flow, covered at the beginning of chapter 3 , now follows. The first moment of the spectrum of total energy still cascades to smaller wavenumbers, but total energy now includes potential energy. The cascade is slower because at scales larger than the deformation radius, potential vorticity is dominated by vortex stretching rather than relative vorticity, and it is the latter that effects nonlinear scale transfer. Nevertheless, a cascade dominated by vortex merger does indeed take place in an f-plane freely evolving experiment we show for illustrative purposes (figure 6-24). The experiment in 624 was initialized with a forced-dissipated f-plane solution from chapter 3 and run with a wavenumber filter small-scale dissipation, resulting in only two percent loss of total eddy energy over the integration. There is no asymmetry between cyclones and anticyclones in either the initial or final state. In the next section we initialize 1.5 layer experiments with asymmetric vortex solutions and hyperviscosity as smallscale dissipation.

Freely decaying turbulence in the equivalent barotropic model has been examined previously (Larichev and McWilliams 1991). In that paper the quadratic invariant was developed through multiplication of the full potential vorticity, and it was noted that it was difficult to use this invariant in a cascade argument. They postulated that the potential energy spectrum would show a cascade. Here we have proven a cascade in the total energy spectrum. Larichev and McWilliams (1991) also noted the slowdown of the cascade as compared to cascades in purely two-dimensional turbulence, but concluded that a slow cascade to large scales does take place. They presented results from several experiments, with different values of $R_{d}$. The integration presented here is much longer than any of theirs was, thus allowing the cascade to proceed to finality. 


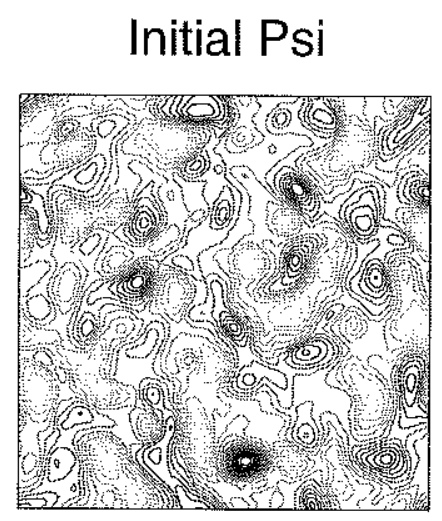

Final Psi

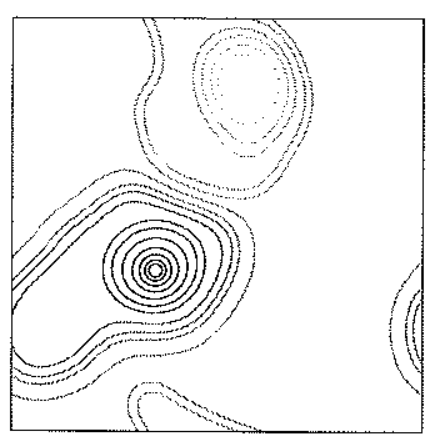

Psi at 190.8 Years

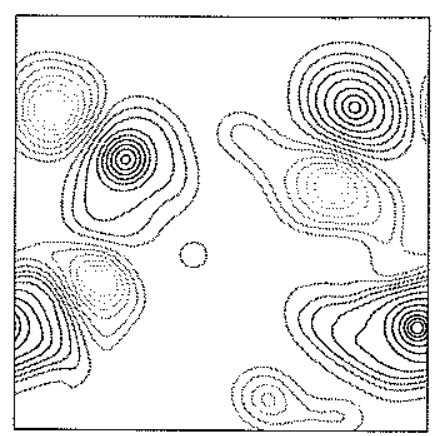

Etotal Length Scale

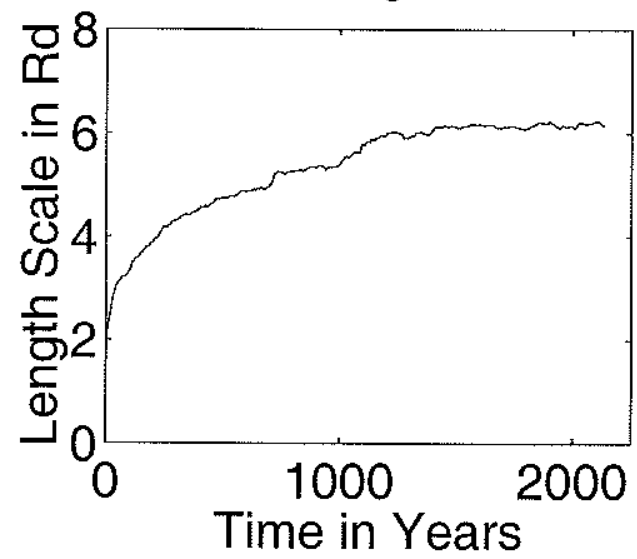

Figure 6-24: Results of a 256 squared wavenumber filter f-plane freely evolving onelayer $Q G$ experiment with a deformation radius present. Eddy length scales are measured as reciprocals of moments of energy spectra. 


\subsubsection{Freely decaying equivalent barotropic turbulence ini- tialized by vortex solutions}

Through initialization of a one-layer experiment with the top layer streamfunction of the nominal vortex experiment, we can study how vortices evolve in the absence of forcing, dissipation, and interactions with a bottom layer. First we present results from an f-plane experiment (figure 6-25). In this case, 41 percent of the initial eddy energy has been lost. This experiment is still ongoing-it seems to be heading towards a state dominated by one large cyclonic vortex. Figure 6-26 indicates that the cascade takes place more quickly on a beta plane $\left(\beta=\frac{1}{16} \bullet 2 \bullet 10^{-11} \mathrm{~m}^{-1}\right.$ $\mathrm{s}^{-1}$, as in the nominal forced-damped vortex solution). Here 26 percent of the initial energy is lost. Very similar results to the beta plane result were obtained in an experiment in which the total background PV gradient was set to equal the total upper layer mean PV gradient in the nominal forced-dissipated experiment. The presence of background PV gradients in one-layer freely decaying experiments seems to accelerate the cascade towards fewer vortices and larger scales. Note that the presence of gradients in one layer, even if they are different in the $\mathrm{x}$ and $\mathrm{y}$ directions (as in the experiment we explained but did not show), does not generate either energy or enstrophy, so the argument we gave in the preceding section for a cascade in total energy length scale is unchanged.

\subsubsection{Two-layer freely decaying turbulence initialized by vor- tex solutions}

In this section, we report on two-layer freely-decaying experiments initialized with the nominal vortex solution. Figures 6-27 and 6-28 display the f-plane results. Barotropization takes place, but the baroclinic mode retains sufficient energy to prevent the cascade to larger scales from taking place rapidly. Forty-seven percent of the initial energy is retained. 


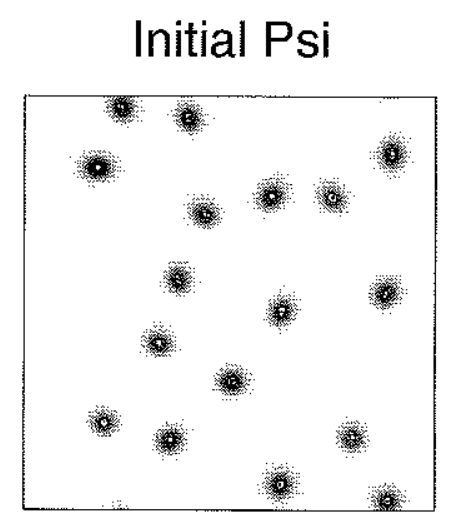

Final Psi

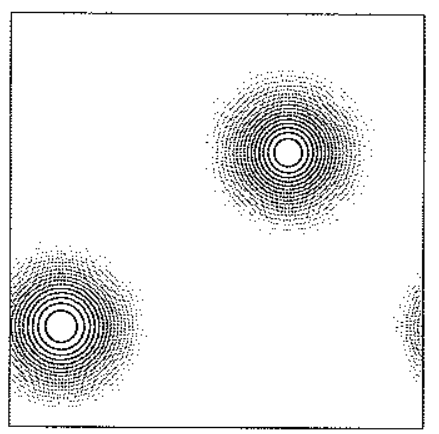

\section{Etotal Length Scale}

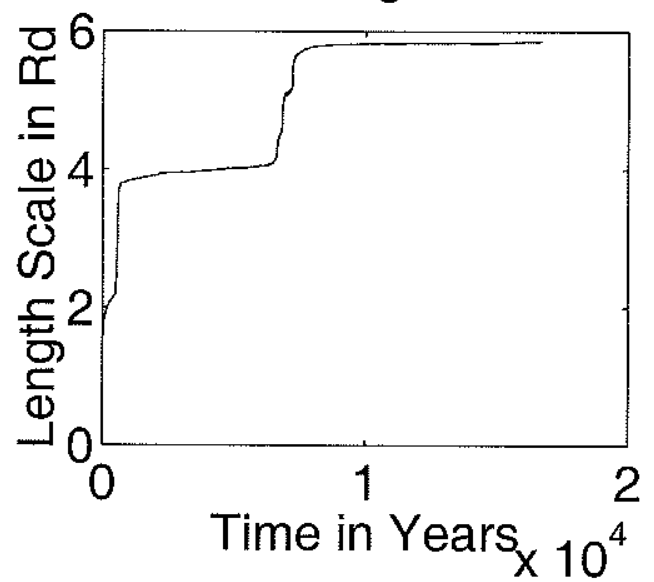

Figure 6-25: Results of a 128 squared hyperviscous f-plane freely decaying one-layer experiment with a deformation radius present, initialized with the top layer nominal vortex solution. 


\section{Initial Psi}

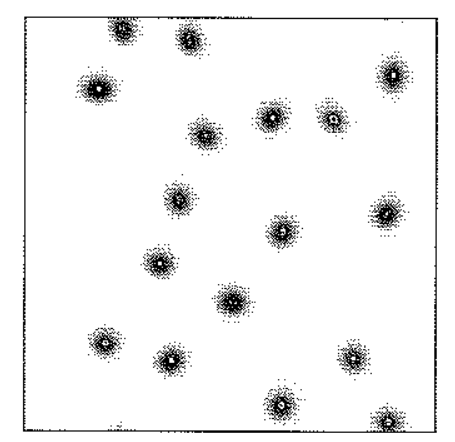

Final Psi

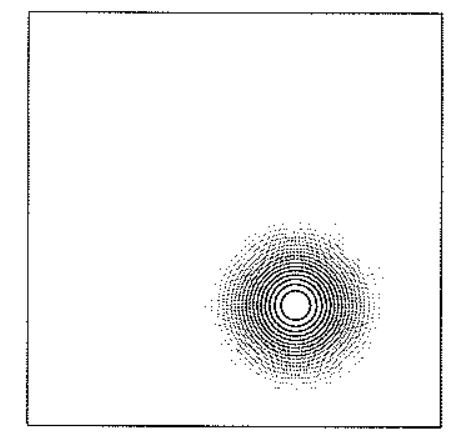

Etotal Length Scale

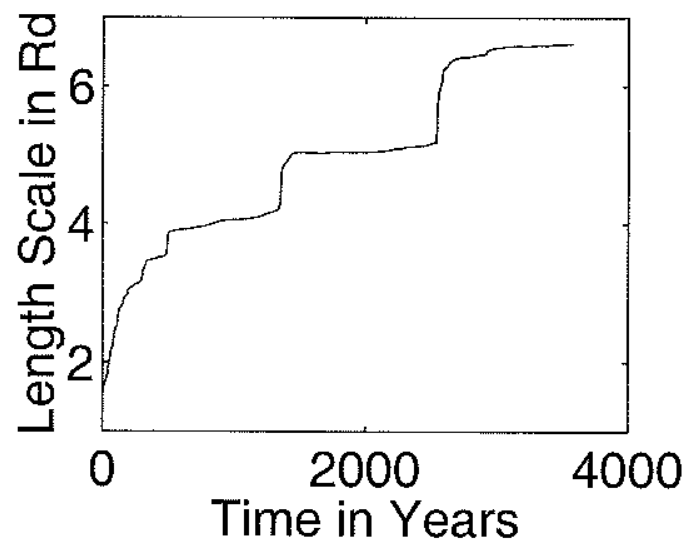

Figure 6-26: Results of a 128 squared hyperviscous freely decaying one-layer QG experiment with a deformation radius present, initialized with the top layer nominal vortex solution, and run on a beta plane. (Beta has the same value as in the nominal vortex experiment, that is, $\frac{1}{16} \bullet 2 \bullet 10^{-11} \mathrm{~m}^{-1} \mathrm{~s}^{-1}$.) 


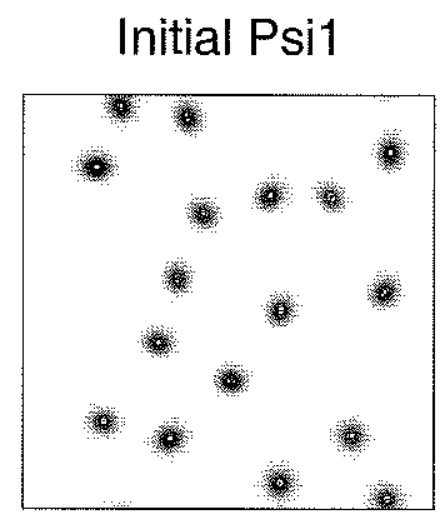

Final Psi1

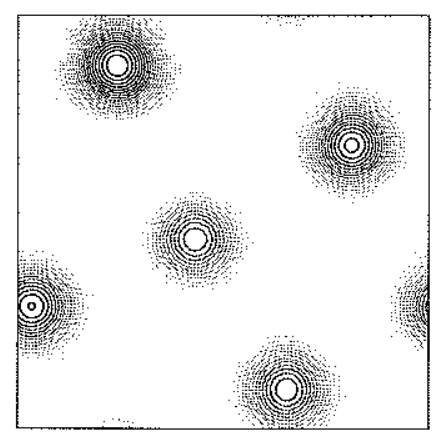

Initial Psi2

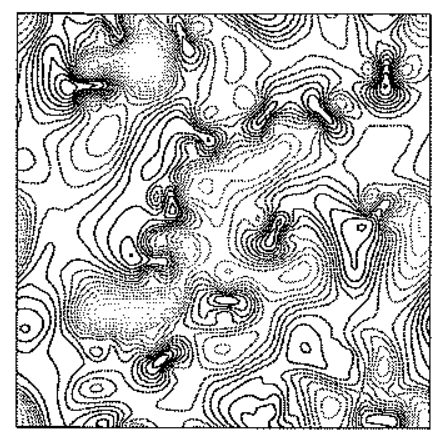

Final Psi2

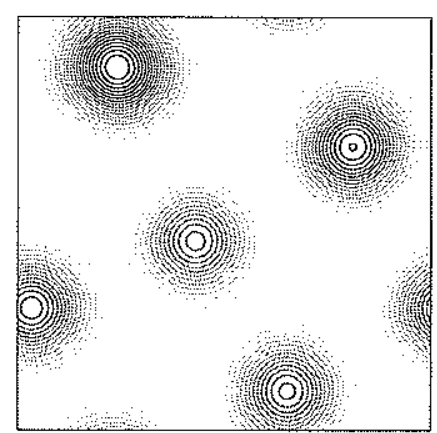

Figure 6-27: Results of a 128 squared hyperviscous freely decaying f-plane two-layer experiment, initialized with the nominal vortex solution. 

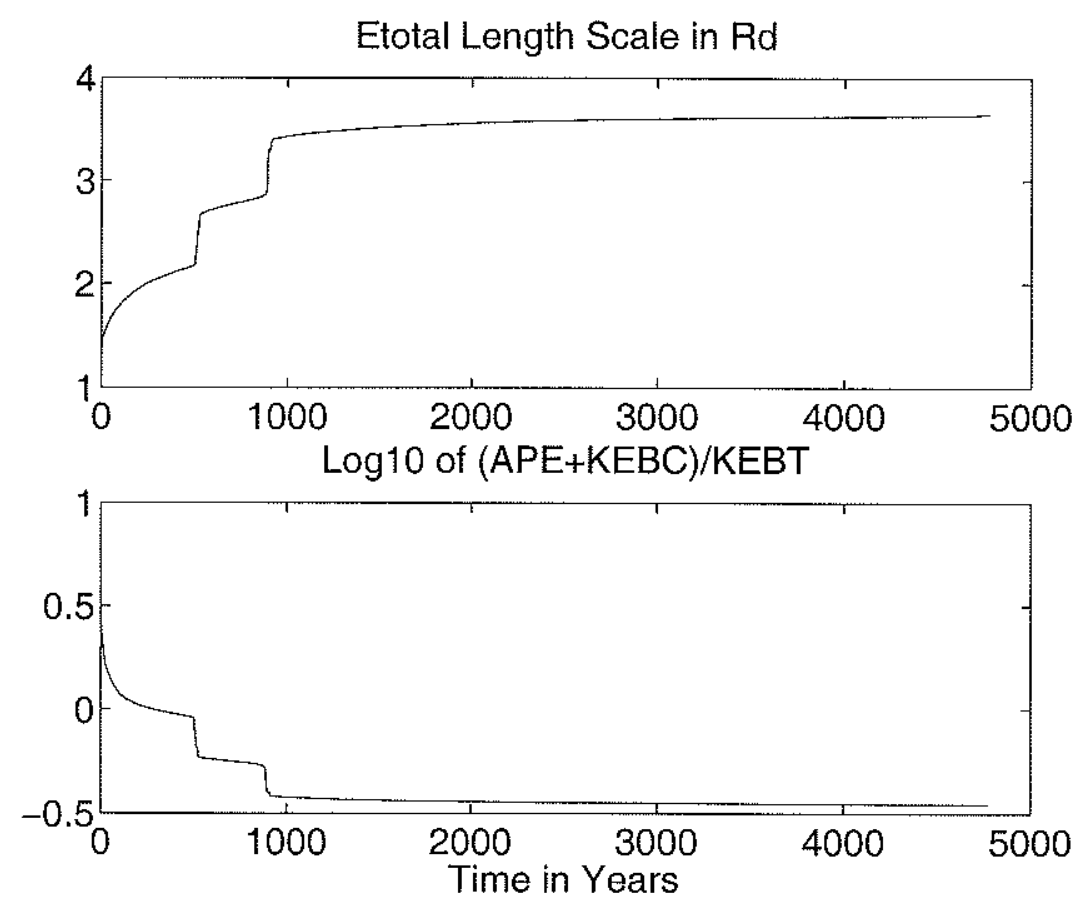

Figure 6-28: Results of a 128 squared hyperviscous freely decaying f-plane two-layer experiment, initialized with the nominal vortex solution.

Freely decaying results on a beta-plane (where beta takes on the same value as in the nominal vortex experiment) are shown in figure 6-29. This experiment retained eighty percent of its total energy. Barotropization and the inverse cascade are rapid and nearly complete, and the vortices have disappeared.

An interesting contrast is provided in the next experiment, shown in figure 6-30. Here the four background mean PV gradients-one in the $\mathrm{x}$ direction and one in the $\mathrm{y}$ direction, in each layer-equal those in the nominal forced-dissipated case. However, they are set as planetary beta-like terms, so they do not generate energy as do shear gradients. It is run for the same amount of time as the preceding experiment, but only thirty percent of the energy is retained. Energy decay (not shown) shows no sign of stopping, whereas it had clearly stopped in the beta-plane case. The same number of coherent cyclones remains, and the inverse cascade and barotropization processes are held at bay. This experiment probably behaves differently because the presence 
Final Psi1

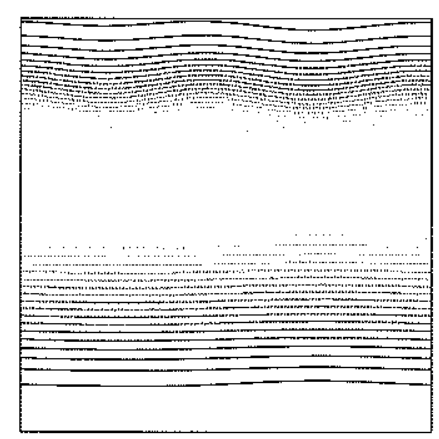

Etotal Length Scale in Rd

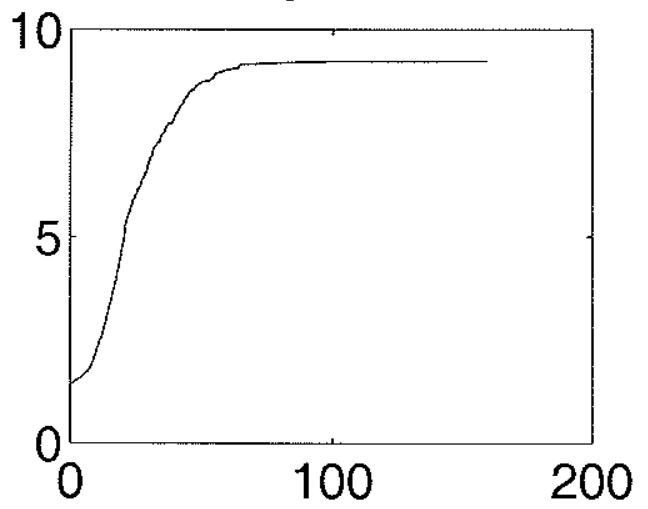

Final Psi2
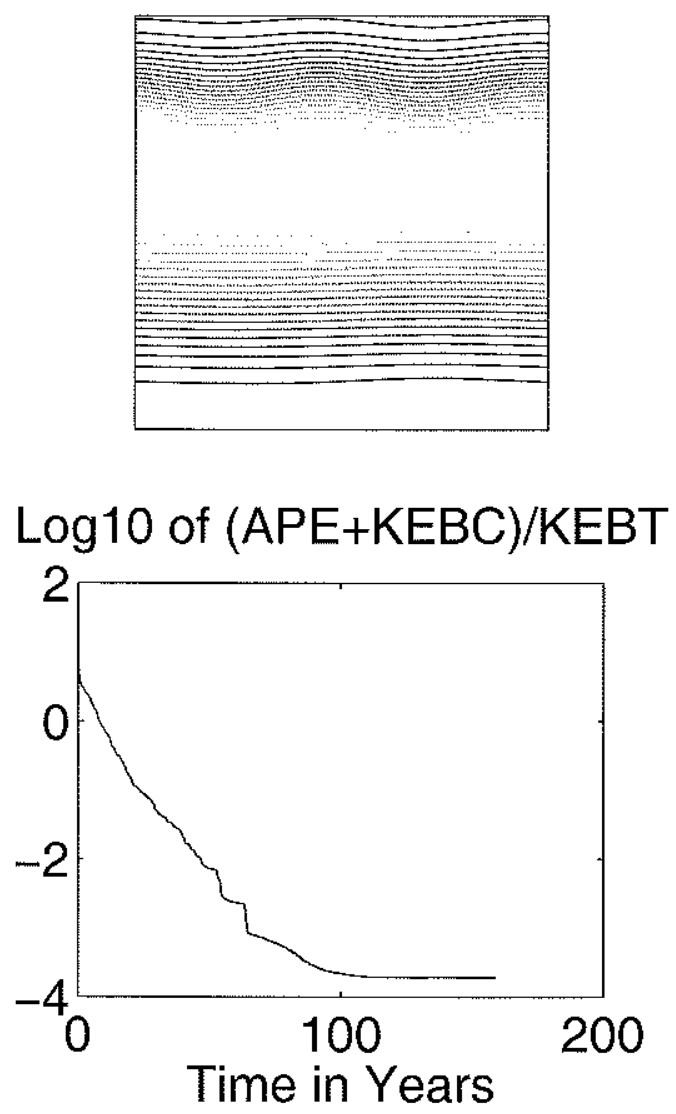

Figure 6-29: Results of a 128 squared hyperviscous freely decaying beta plane (where beta takes on the same value as in the nominal vortex experiment) two-layer experiment, initialized with the nominal vortex solution as in 6-27. 
of different betas in the lower and upper layers generates enstrophy. In that regard, this particular experiment is analogous to experiments with bottom topography, which also introduces betas that are different in the two layers, generates enstropy, and can halt cascades in freely evolving turbulence (Rhines 1977; LaCasce 1996). In the present experiment twenty seven percent of the initial potential enstrophy is retained. In contrast, the beta plane experiment retained less than one percent of its initial enstrophy. It remains to be seen whether this experiment will have any bearing on the halting of the cascade in the forced-dissipated case. Layer PV gradients are equal in the two cases. But there is a difference between an imposed "betalike" background gradient (as we have done here) and a shear-induced gradient-for example, the latter, unlike the former, can generate energy.

\subsubsection{Two-layer turbulence initialized by vortex solutions and dissipated by bottom Ekman friction}

Figure 6-31 shows the results of a two-layer f-plane integration initialized with the nominal vortex solution and damped by a bottom Ekman friction (dissipation time 193 days) in addition to hyperviscosity. The vortices decohere under the influence of Ekman friction, and then undergo a very slow cascade to larger and fewer features. The final solution does not retain the initial asymmetry. Even when background PV gradients are set up in the way most likely to maintain vortices in the freely decaying case, that is, equal to those in the forced-dissipated case, vortices disappear under the influence of Ekman friction (figure 6-32).

The previous experiments indicate that vortices quickly disappear in the presence of Ekman friction, if there is no forcing. Figure 6-33 displays results of a beta-plane experiment initialized with the nominal vortex solution, in which a westward shear is imposed and adjusted so that $\frac{\partial \bar{q}_{1}}{\partial y}=0$. Thus, there is a source of energy, but not a strong one. There is still a signature of the vortices after a long integration, unlike in the previous two cases. Vortex disintegration under Ekman friction can be held 
Final Psi1

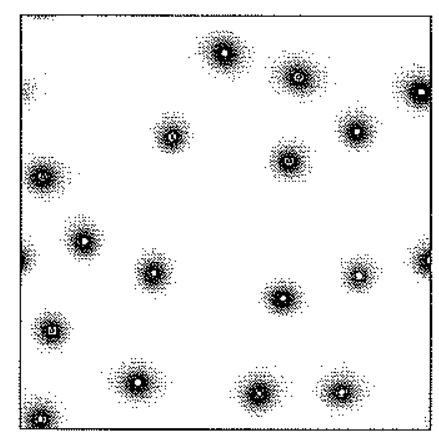

Etotal Length Scale in $\mathrm{Rd}$

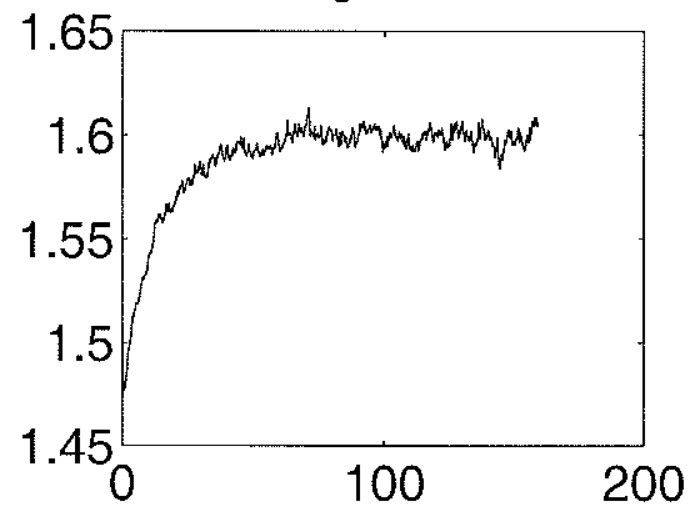

Final Psi2

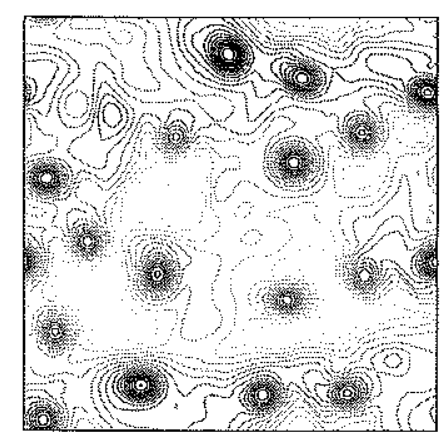

Log10 of (APE+KEBC)/KEBT

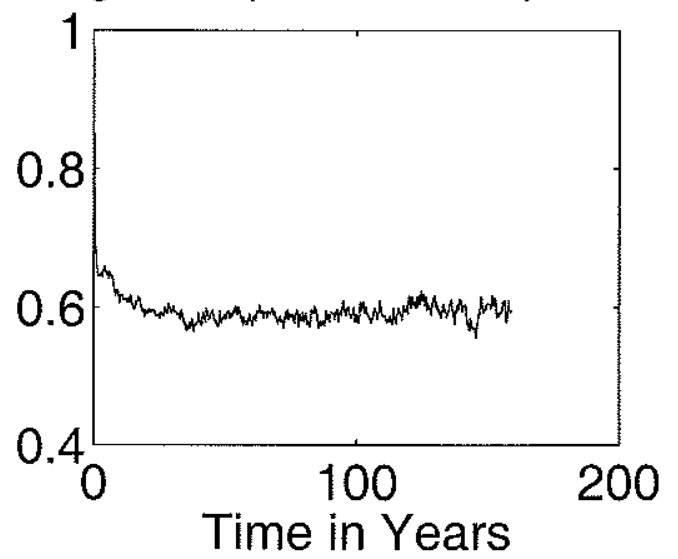

Figure 6-30: Results of a 128 squared hyperviscous freely decaying two-layer experiment, initialized with the nominal vortex solution, in which the layer PV gradients are set to equal those in the nominal forced-dissipated solution. 
Final Psi1

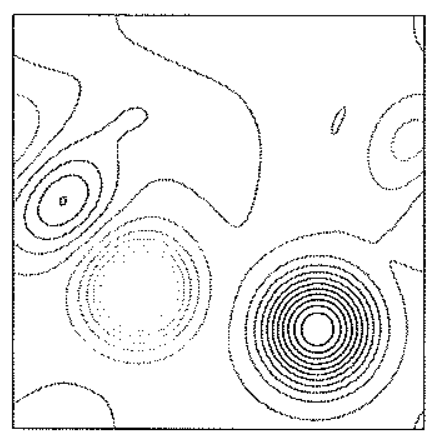

Etotal Length Scale in Rd

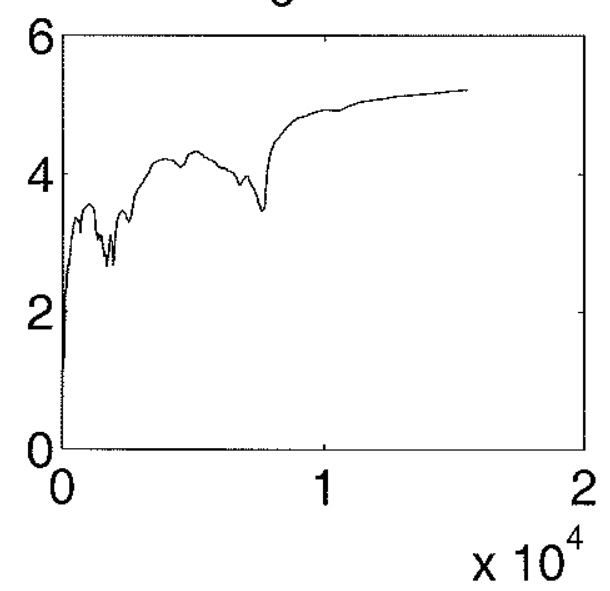

Log10 of Etotal in $\mathrm{cm}^{2}$ per $\mathrm{s}^{2}$
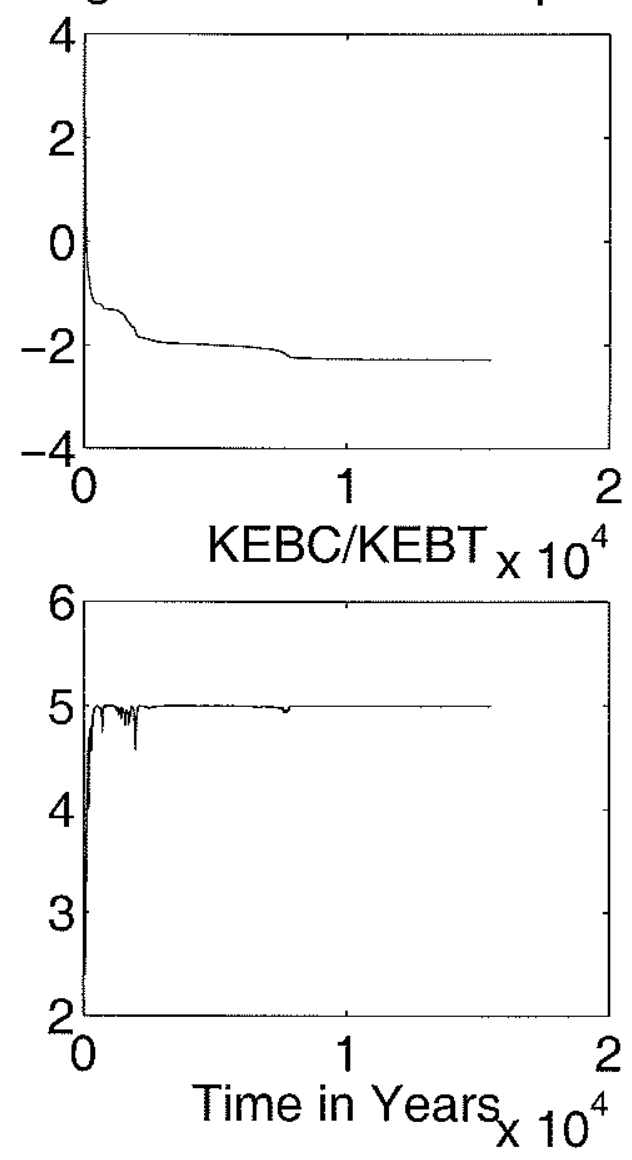

Figure 6-31: Results of a 128 squared hyperviscous f-plane two-layer experiment, initialized with the nominal vortex solution and dissipated with a 193.4 day bottom Ekman friction. 
Final Psi1

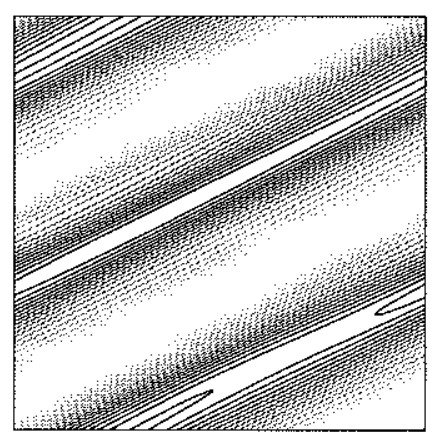

Etotal Length Scale in Rd

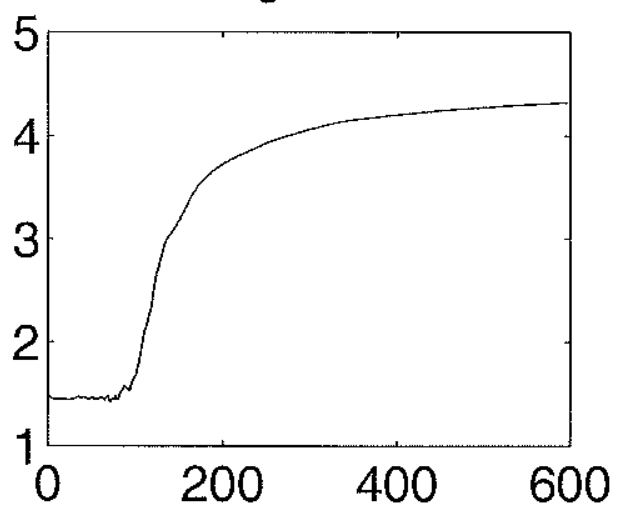

Log10 of Etotal in $\mathrm{cm}^{2}$ per $\mathrm{s}^{2}$
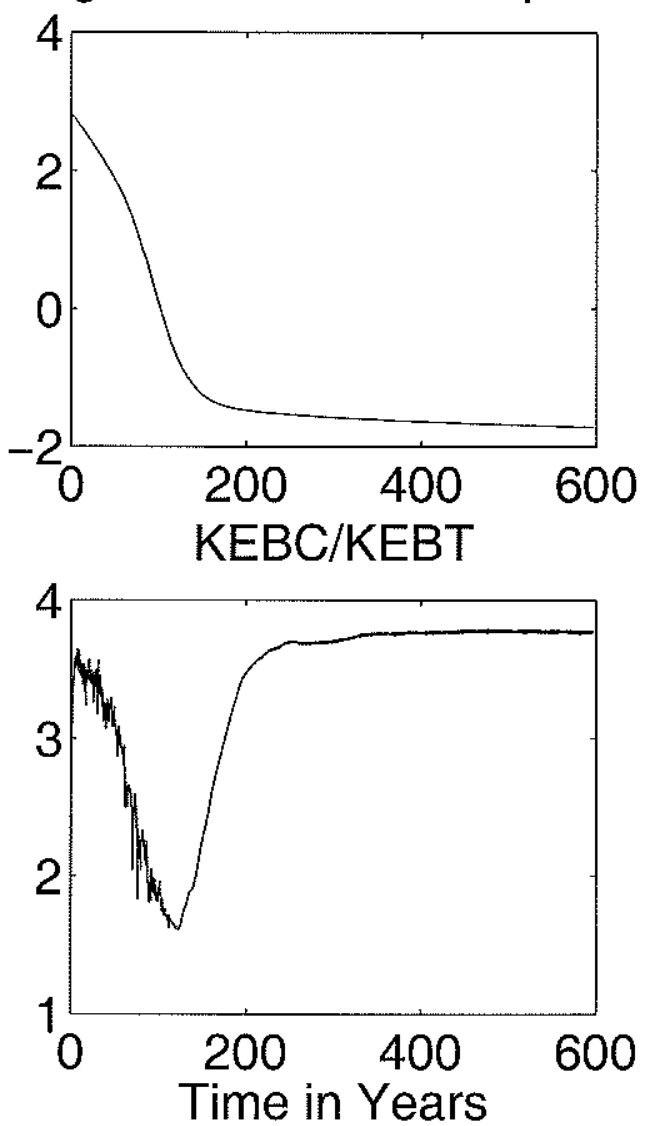

Figure 6-32: Results of a 128 squared hyperviscous two-layer experiment, initialized with the nominal vortex solution and dissipated with a 193.4 day bottom Ekman friction. Here layer PV gradients equal those in the nominal forced-dissipated solution. 
at bay for a long time with even a weak shear. From the forced-dissipated results we know that a strong shear will maintain vortices indefinitely. This indicates that extraction of energy from the mean shear via baroclinic instability is an essential feature of the vortex regime.

\subsection{Dominant balance of the vortex solutions}

In the last section we learned that the vortices we examine in this chapter, like other QG solutions, tend to cascade to the barotropic mode and to larger scales (albeit, very slowly in some cases) in the absence of forcing, Ekman friction, and of background PV gradients which are unequal between layers. We also learned that vortices disappear under the action of Ekman friction, unless it is balanced by forcing. In this section we perform a preliminary investigation of the dynamics of the vortex regime with the nominal forcing and dissipations present. We begin with figure 6-34, which displays scatter plots of nondimensional $\psi_{1}$ versus $q_{1}$ for three of the vortex solutions. All show a tight correlation between the two quantities. Scatter plots of $\psi_{1}$ and $q_{1}$ for four other solutions examined in this thesis are shown in 6-35. The relationship between $\psi_{1}$ and $q_{1}$ is clearly weaker in these other cases. Figures 6-36 through 6-42 show contour plots of $\psi_{1}, q_{1}$, and $J\left(\psi_{1}, q_{1}\right)$ for the same seven experiments. The isolated nature of $\psi_{1}$ and $q_{1}$ in the vortex solutions shows up in the Jacobian as well. The Jacobians of the other solutions are less isolated and orderly in appearance. The organization and coherence in the Jacobian of the vortex solution compared with the other solutions in this dissertation is the likely reason that the vortices persist as coherent features longer than structures in other parts of the thesis. It also is the likely reason that the inverse cascade is absent in the vortex solutions; the Jacobian in the vortex case keeps the structures coherent rather than scatters energy into and out of them. The Jacobians of the $R_{\beta}=12$ experiment in figure 6-40 and of the viscous f-plane solutions in figure 6-42 show some amount of 
Final Psi1

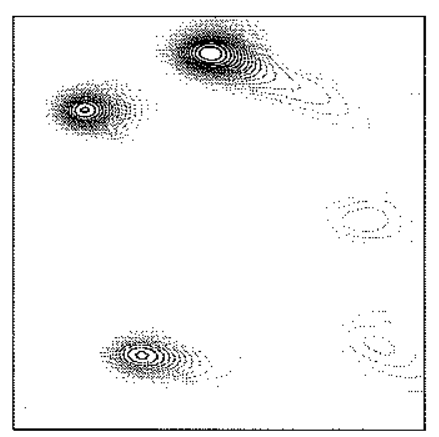

Etotal Length Scale in Rd

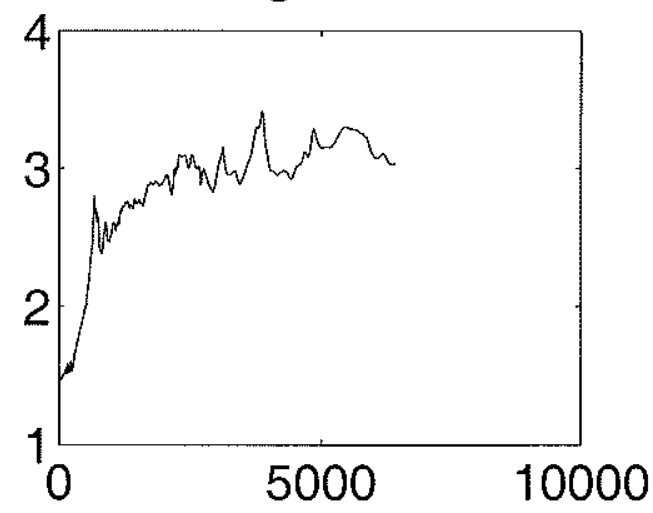

Log10 of Etotal in $\mathrm{cm}^{2}$ per $\mathrm{s}^{2}$
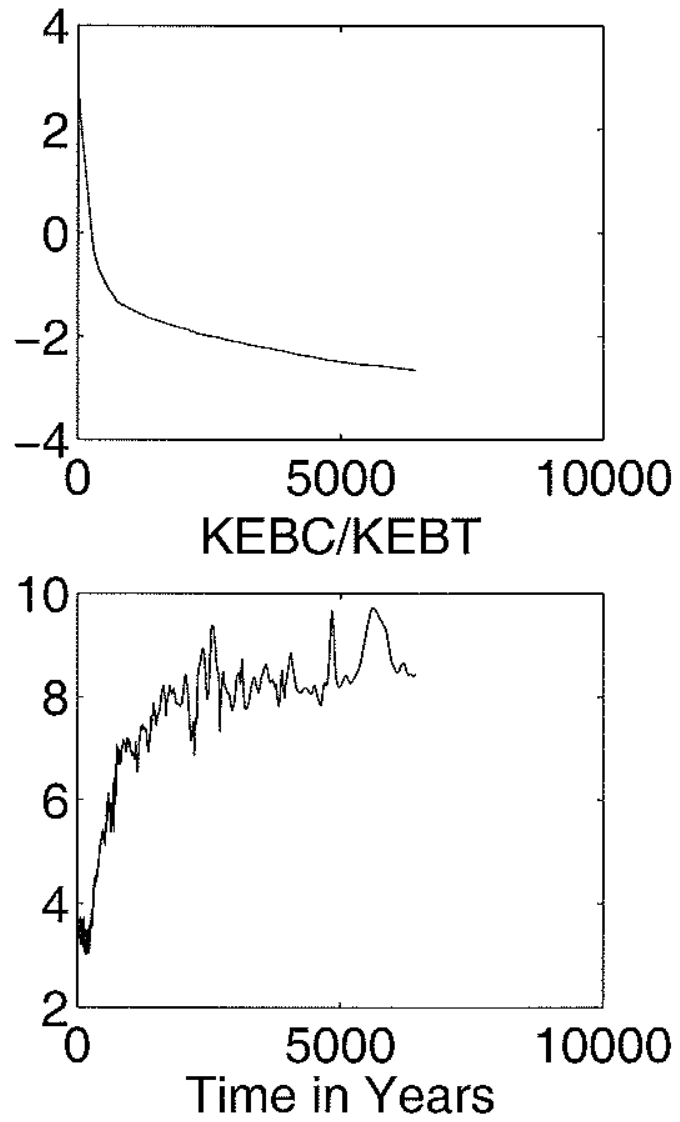

Figure 6-33: Results of a 128 squared hyperviscous two-layer beta plane (where beta is the same as in the nominal vortex experiment, that is, nominal beta over 16) experiment, initialized with the nominal vortex solution and dissipated with a 193.4 day bottom Ekman friction, in which a westward shear is adjusted so that $\frac{\partial \bar{q}}{\partial y}=0$. 
organization, consistent with the persistent fronts seen in those solutions. The fact that $\psi_{1}$ and $q_{1}$ are not well correlated in those cases may be related to the fact that these fronts undergo large meanders over time. In that sense, these fronts are less stable features than the vortices.

The caption of each figure lists a quantity which we call the "cancellation ratio", defined by:

$$
\text { cancellation ratio }=\frac{\left\langle\left(u_{1} \frac{\partial q_{1}}{\partial x}+v_{1} \frac{\partial q_{1}}{\partial y}\right)^{2}\right\rangle}{\left.<\left(u_{1} \frac{\partial q_{1}}{\partial x}\right)^{2}+\left(v_{1} \frac{\partial q_{1}}{\partial y}\right)^{2}\right\rangle},
$$

where brackets denote a spatial average over the domain for the snapshot in question. A small cancellation ratio means that the sum of the individual terms in the Jacobian is small compared to the individual terms themselves, meaning that a dominant nonlinear balance of the form $J(\psi, q) \approx 0$ is likely. The ratio is less than one for all of the experiments but is smallest in the vortex solutions. Only the viscous f-plane experiment has a cancellation ratio that approaches that of the hyperviscous vortex solution, which in turn has a cancellation ratio an order of magnitude larger than that of the wavenumber filter solutions. The greater degree of cancellation in the wavenumber filter solutions is likely due to their more circular nature. 

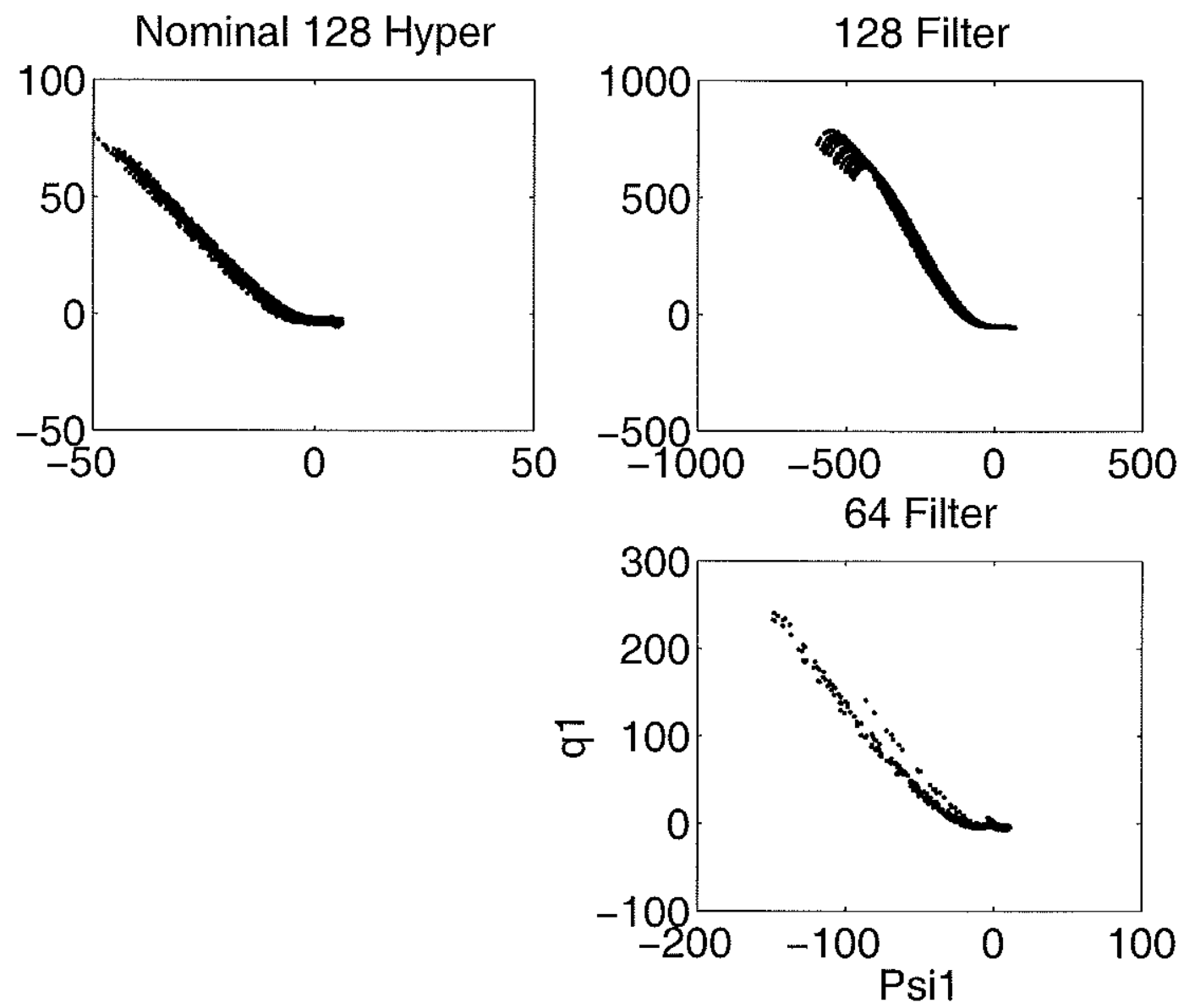

Figure 6-34: Scatter plots of nondimensional upper layer fluctuation PV versus streamfunction, for three of the vortex solutions. Recall that the 128 squared filter result has not yet equilibrated. 

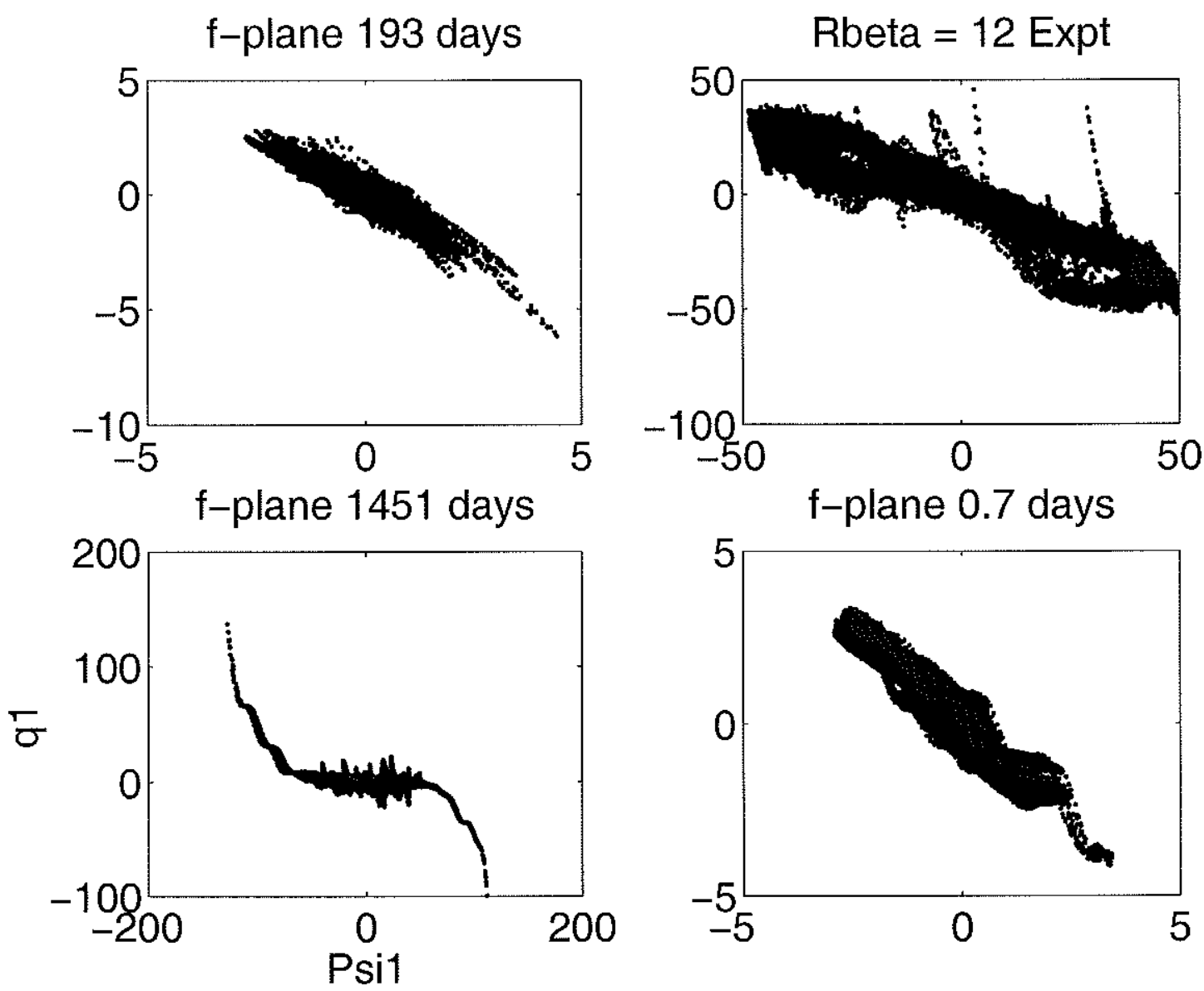

Figure 6-35: Scatter plots of nondimensional upper layer fluctuation PV versus streamfunction, for the hyperviscous f-plane experiment from section 5.3, two wavenumber filter $\delta=0.2$ f-plane bottom-friction solutions from chapter 3 , and the $R_{\beta}=12$ experiment from section 5.3 . 

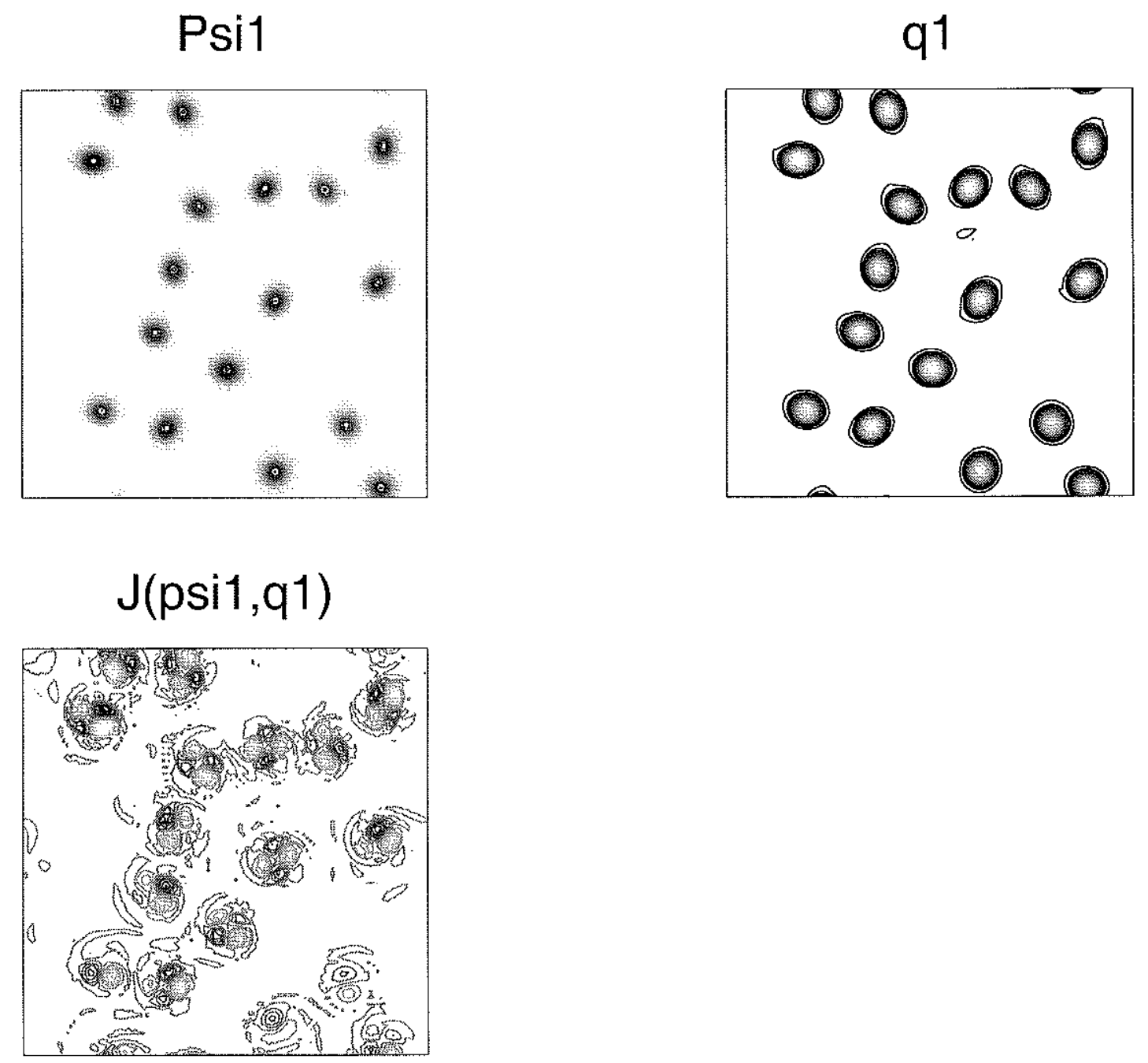

Figure 6-36: Contours of $\psi_{1}, q_{1}$, and $J\left(\psi_{1}, q_{1}\right)$ for snapshot of 128 squared hyperviscous nominal vortex solution. The "cancellation ratio" (see text for details) is 0.0018 . 

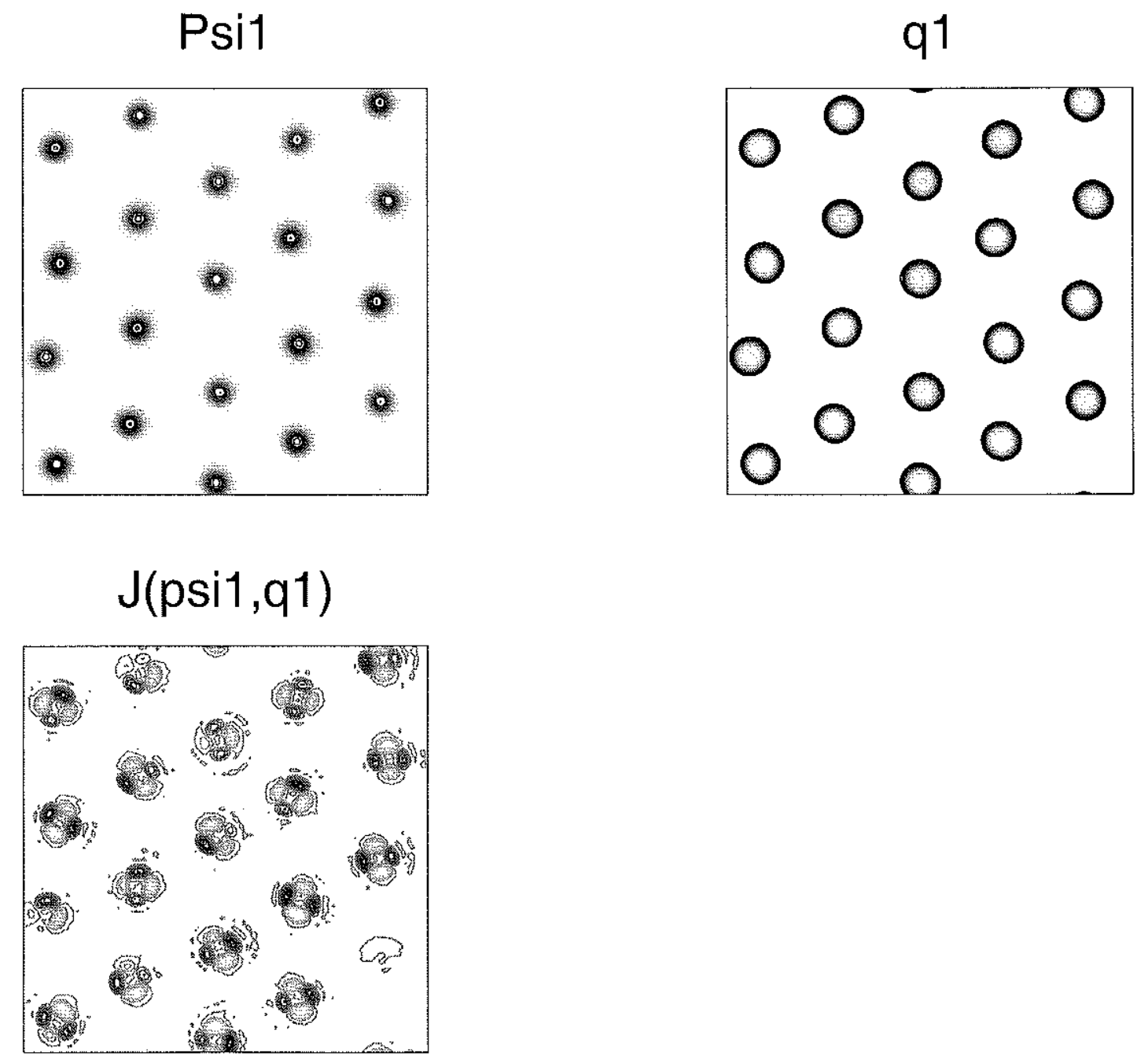

Figure 6-37: Contours of $\psi_{1}, q_{1}$, and $J\left(\psi_{1}, q_{1}\right)$ for snapshot of 128 squared unequilibrated wavenumber filter vortex solution. The cancellation ratio is 0.00015 . 

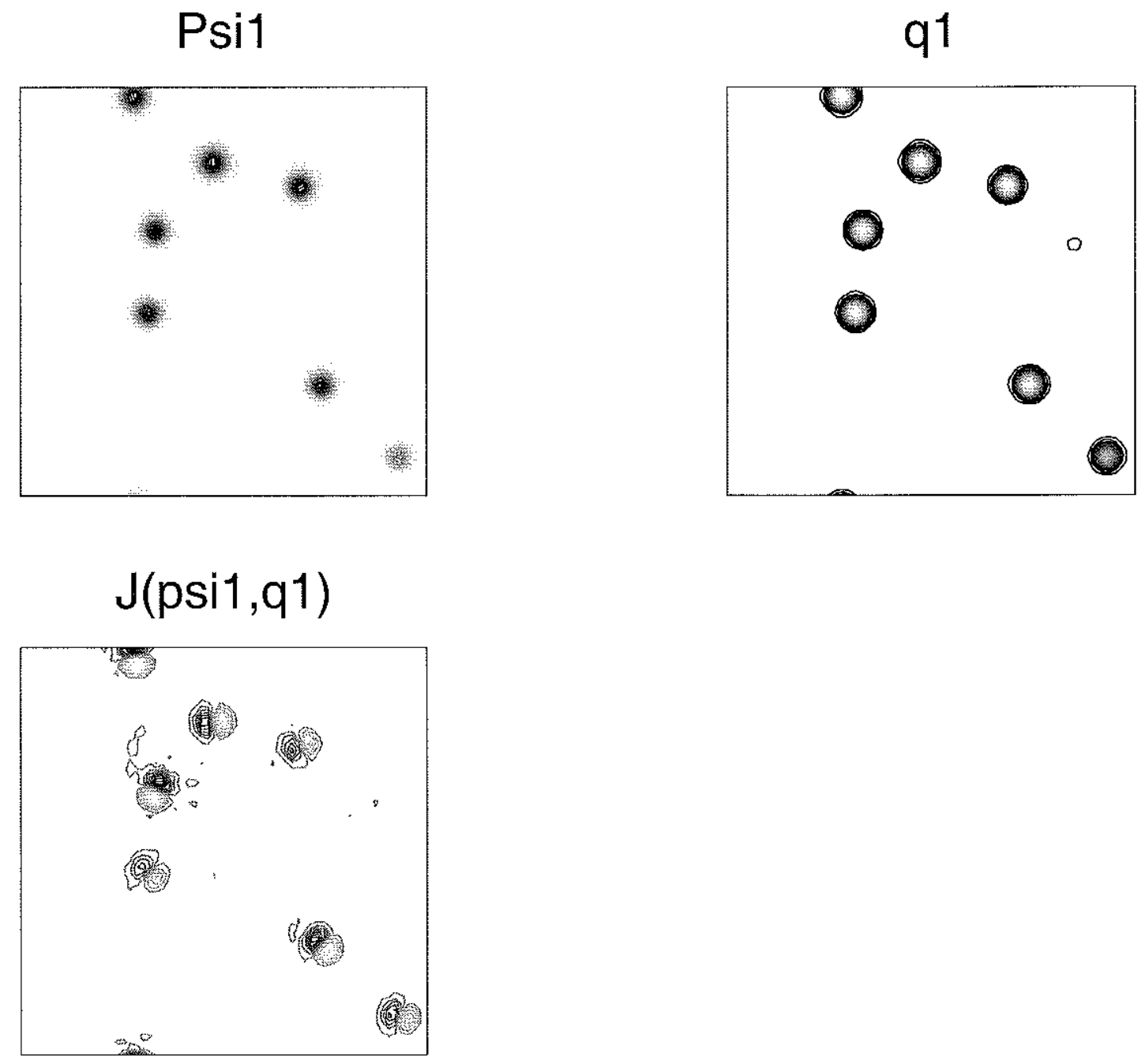

Figure 6-38: Contours of $\psi_{1}, q_{1}$, and $J\left(\psi_{1}, q_{1}\right)$ for snapshot of 64 squared wavenumber filter vortex solution. The cancellation ratio is 0.00023 . 


\section{Psi1}

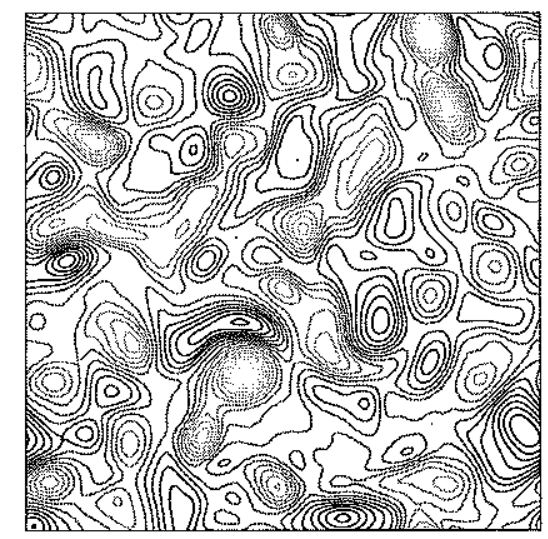

\section{$J(p s i 1, q 1)$}

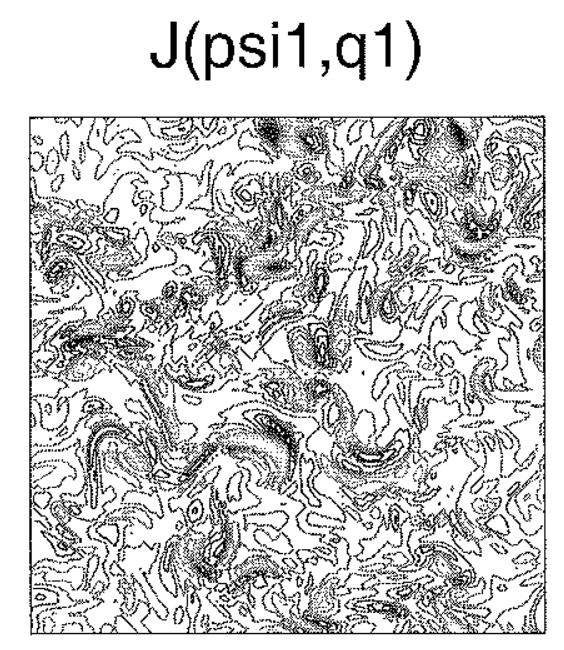

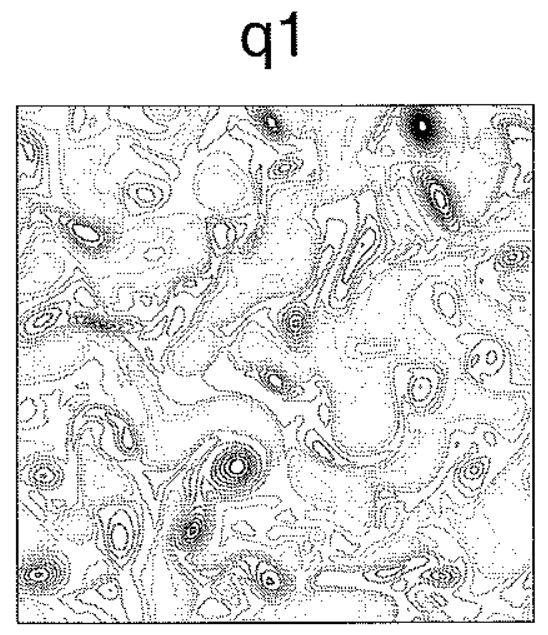

Figure 6-39: Contours of $\psi_{1}, q_{1}$, and $J\left(\psi_{1}, q_{1}\right)$ for snapshot of 128 squared hyperviscous 193.4 day bottom friction $\delta=0.2$ f-plane solution from section 5.3. The cancellation ratio is 0.0615 . 
Psi1

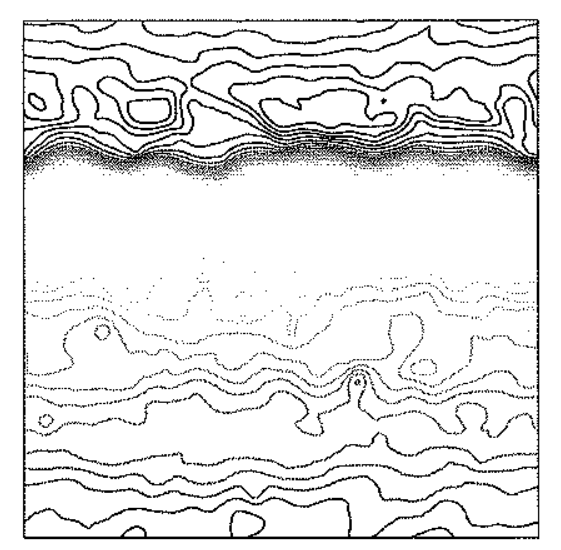

\section{$J(p s i 1, q 1)$}

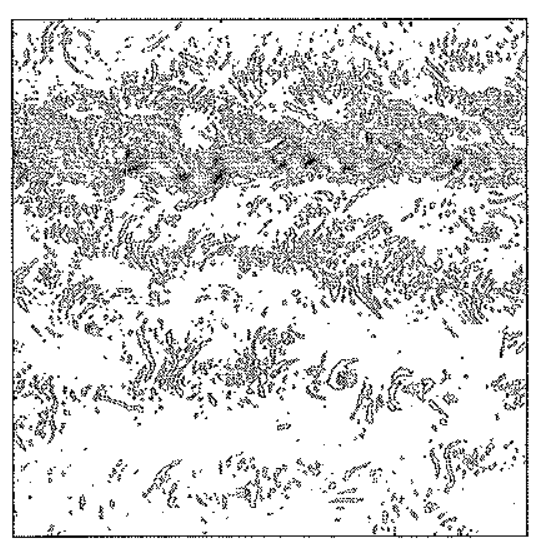

q1

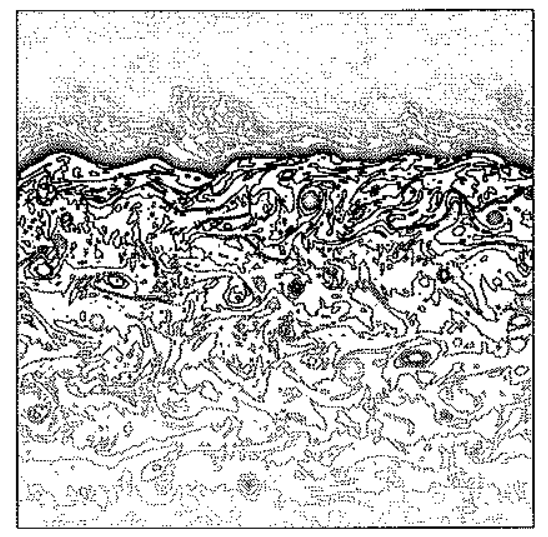

Figure 6-40: Contours of $\psi_{1}, q_{1}$, and $J\left(\psi_{1}, q_{1}\right)$ for snapshot of 256 squared wavenumber filter $R_{\beta}=12$ solution from section 5.3 . The cancellation ratio is 0.206 . 


\section{Psi1}

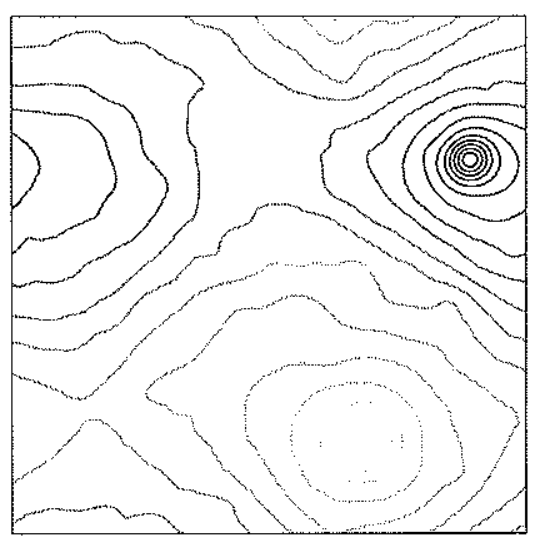

$J(p s i 1, q 1)$

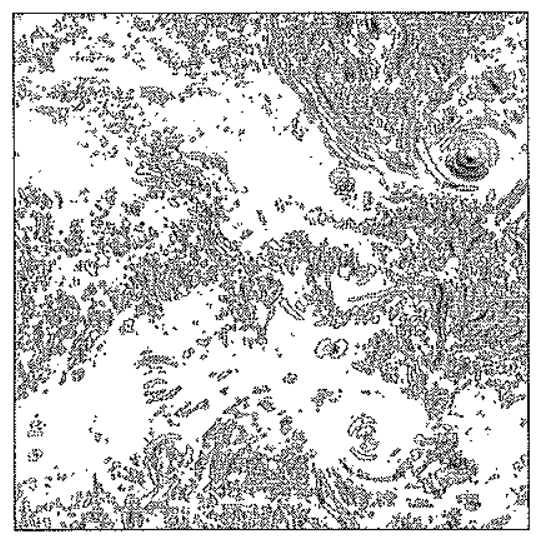

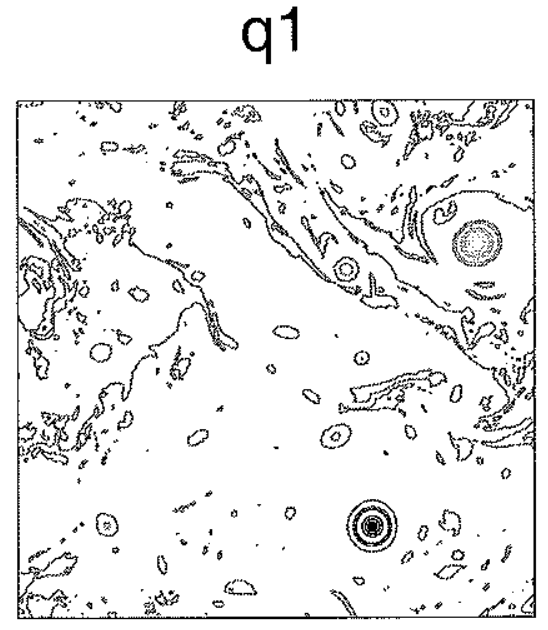

Figure 6-41: Contours of $\psi_{1}, q_{1}$, and $J\left(\psi_{1}, q_{1}\right)$ for snapshot of 256 squared wavenumber filter 1451 day bottom friction $\delta=0.2$ f-plane solution from chapter 3 . The cancellation ratio is 0.0772 . 

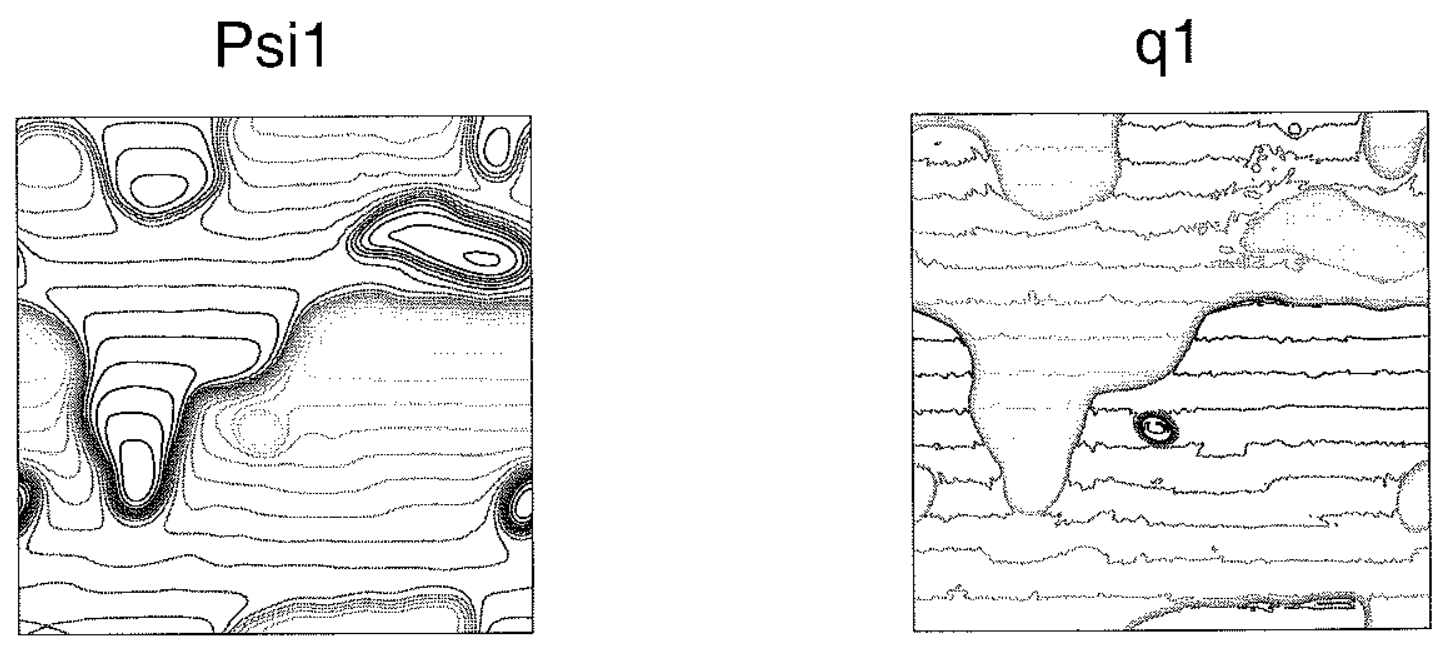

$J(p s i 1, q 1)$

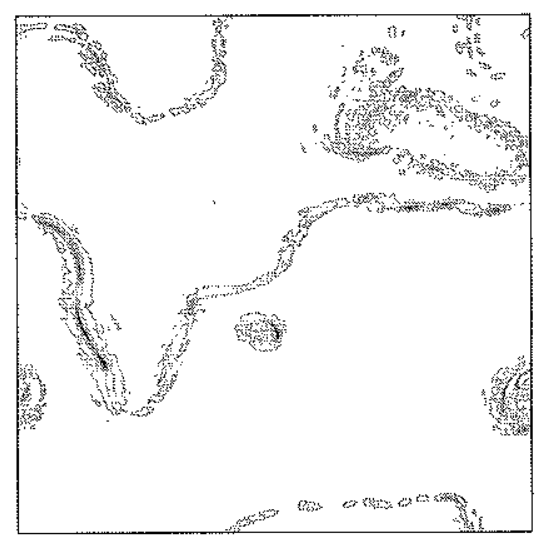

Figure 6-42: Contours of $\psi_{1}, q_{1}$, and $J\left(\psi_{1}, q_{1}\right)$ for snapshot of 256 squared wavenumber filter 0.7 day bottom friction $\delta=0.2$ f-plane solution from chapter 3 . The cancellation ratio is 0.0206 . 
The tightness of the $\psi_{1} / q_{1}$ scatter plots, and the small cancellation ratios, for the vortex experiments point towards the possibility of a balance dominated by the Jacobian. To establish this more firmly we compare terms in the upper layer PV equation. Figure 6-43 plots $J\left(\psi_{1}, q_{1}\right)$ alongside $J\left(\overline{\psi_{1}}, q_{1}\right)$ and $J\left(\psi_{1}, \overline{q_{1}}\right)$, and $\frac{\partial q_{1}}{\partial t}$, the last having been calculated from the small-scale dissipation minus the three Jacobians. Isolated coherent structures are evident in all three Jabobians, as well as the tendency term, which strongly resembles $-J\left(\psi_{1}, q_{1}\right)$. Rms values of these terms are tabulated in table 6.1, which indicates that the dominant balance in the upper layer is:

$$
\frac{\partial q_{1}}{\partial t}+J\left(\psi_{1}, q_{1}\right) \approx 0
$$

This is reminiscent of modon/soliton solutions (c.f. Stern 1975; Larichev and Reznik 1976; Flierl et al. 1980). Dissipation and terms involving the mean flow enter in at higher order. Lower layer fields $\psi_{2}, q_{2}$, and $J\left(\psi_{2}, q_{2}\right)$ are contoured in figure 6-44 alongside a scatter plot of $\psi_{2}$ and $q_{2}$. Table 6.2 demonstrates that a similar balance holds in the lower layer:

$$
\frac{\partial q_{2}}{\partial t}+J\left(\psi_{2}, q_{2}\right) \approx 0
$$

The scatter plot of $q_{2}$ versus $\psi_{2}$ indicates that there is not a close relationship between these quantities. Thus $u_{2} \frac{\partial q_{2}}{\partial x}$ and $v_{2} \frac{\partial q_{2}}{\partial y}$ are smaller than $J\left(\psi_{2}, q_{2}\right)$. The individual terms in the Jacobian are not organized to cancel each other out as in the top layer. However, the Jacobian is still organized and coherent in the lower layer. This is because $q_{2}$ is dominated by $\frac{\delta \psi_{1}}{(1+\delta) R_{d}^{2}}$ and hence is still vortical. Since $\psi_{2}$ is small, the lower layer Jacobian is large in regions in which the gradients of $q_{2}$ are large-i.e. in the vortices.

Table 6.3 shows that the mean flow terms are nearly as large as $J\left(\psi_{1}, q_{1}\right)$ in the f-plane solution having the same Ekman friction as the nominal vortex experiment. 
Thus the balance in equation 6.3 does appear to be unique to the vortex regime. In the f-plane case the presence of forcing at lowest order explains the lack of persistent features.

\begin{tabular}{c|c}
\hline \hline Term & Rms Value over Domain \\
\hline \hline$u_{1} \frac{\partial q_{1}}{\partial x}$ & 48.24 \\
\hline$v_{1} \frac{\partial q_{1}}{\partial y}$ & 48.34 \\
\hline$J\left(\psi_{1}, q_{1}\right)$ & 2.87 \\
\hline$\overline{u_{1}} \frac{\partial q_{1}}{\partial x}$ & 0.31 \\
\hline$\overline{v_{1}} \frac{\partial q_{1}}{\partial y}$ & 0.13 \\
\hline$J\left(\overline{\psi_{1}}, q_{1}\right)$ & 0.34 \\
\hline$u_{1} \frac{\partial \overline{q_{1}}}{\partial x}$ & 0.08 \\
\hline$v_{1} \frac{\partial \bar{q}_{1}}{\partial y}$ & 0.19 \\
\hline$J\left(\psi_{1}, \overline{q_{1}}\right)$ & 0.20 \\
\hline$-\nu \nabla q_{1}$ & 0.065 \\
\hline$\frac{\partial q_{1}}{\partial t}$ & 2.87 \\
\hline
\end{tabular}

Table 6.1: Rms value of terms in upper layer PV equation for a snapshot of the nominal 128 squared hyperviscous vortex solution.

The size of the hyperviscous terms in tables 6.1 and 6.2 is not negligible. This, along with the fact that vortex solutions are very sensitive to details of the smallscale dissipation, cause concern that there might be a large amount of energy near unresolved scales. These concerns were reduced by figures such as $6-45$, which plots the upper layer kinetic energy spectrum for the snapshot of the nominal vortex solution. There is not a pile-up of energy at the smallest scales. Spectra of other vortex solutions have been inspected, and no signs of a blue spectrum at small scales have been found. In the nominal solution, bottom Ekman friction accounts for 83 percent of the energy dissipation. A preliminary investigation of other $\delta=0.2$ 


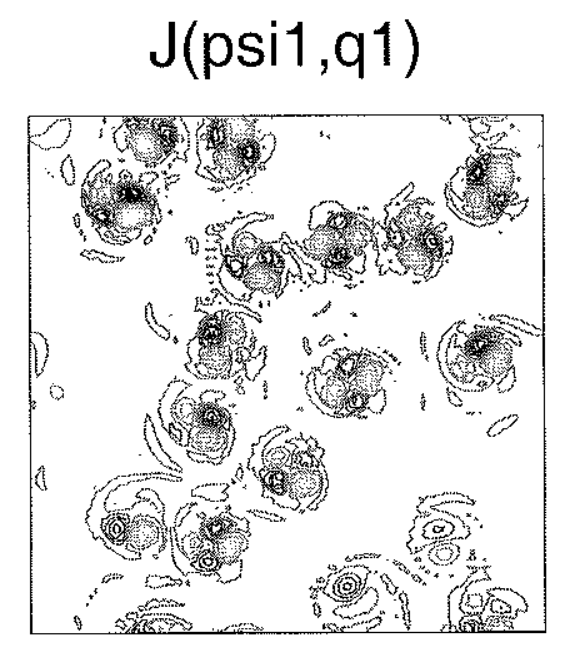

$\mathrm{J}(\mathrm{psi1bar}, \mathrm{q} 1)$

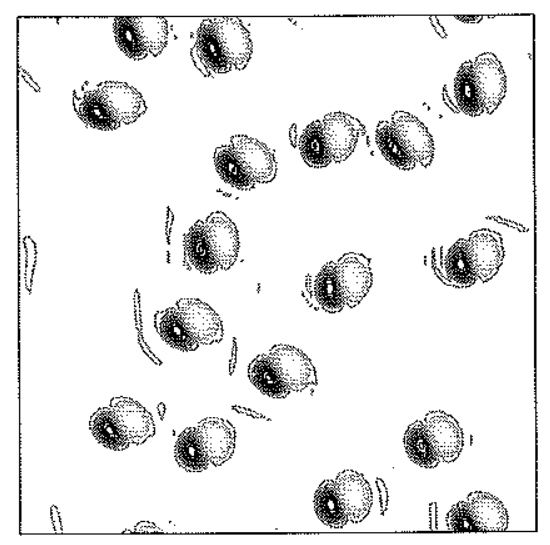

\section{$J(p s i 1, q 1 b a r)$}

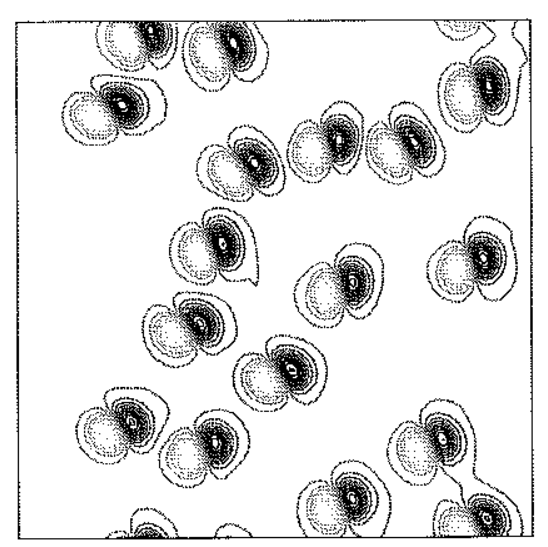

$\mathrm{dq} 1 \mathrm{dt}$

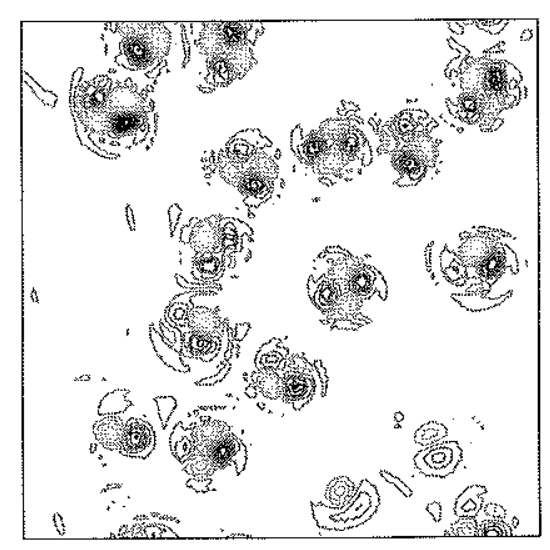

Figure 6-43: Contours of various terms in the upper level PV equation for a snapshot of the nominal 128 squared hyperviscous vortex solution. 

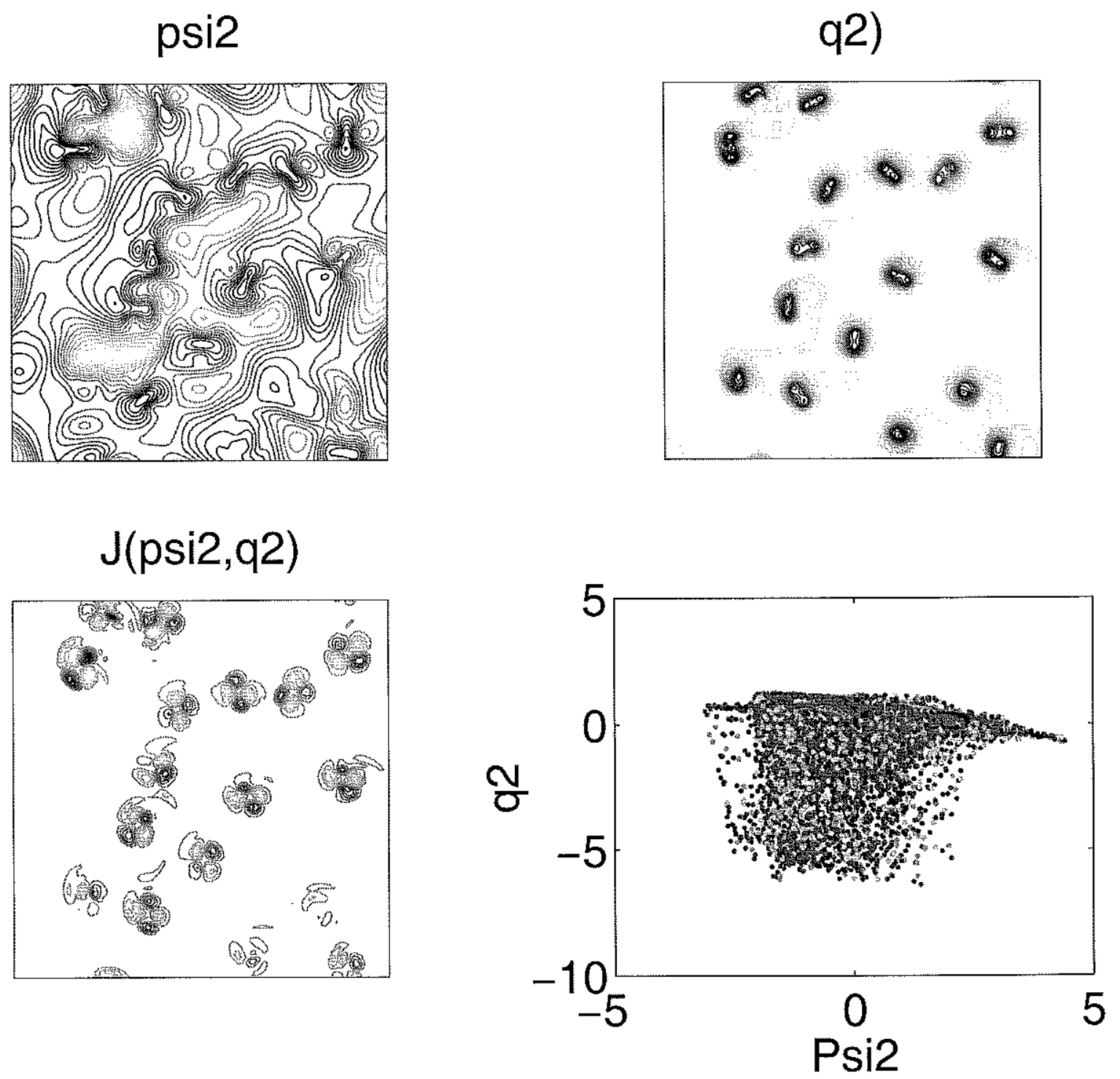

Figure 6-44: Contours of $\psi_{2}, q_{2}$, and $J\left(\psi_{2}, q_{2}\right)$ for a snapshot of the nominal 128 squared hyperviscous vortex solution. The lower right panel displays a scatter plot of $q_{2}$ versus $\psi_{2}$ 


\begin{tabular}{c|c}
\hline \hline Term & Rms Value over Domain \\
\hline \hline$u_{2} \frac{\partial q_{2}}{\partial x}$ & 0.44 \\
\hline$v_{2} \frac{\partial q_{2}}{\partial y}$ & 0.44 \\
\hline$J\left(\psi_{2}, q_{2}\right)$ & 0.66 \\
\hline$\overline{u_{2}} \frac{\partial q_{2}}{\partial x}$ & 0.033 \\
\hline$\overline{v_{2}} \frac{\partial q_{2}}{\partial y}$ & 0.003 \\
\hline$J\left(\overline{\psi_{2}}, q_{2}\right)$ & 0.033 \\
\hline$u_{2} \frac{\partial q_{2}}{\partial x}$ & 0.0015 \\
\hline$v_{2} \frac{\partial q_{2}}{\partial y}$ & 0.0172 \\
\hline$J\left(\psi_{2}, \overline{q_{2}}\right)$ & 0.0172 \\
\hline$-\nu \nabla^{6} q_{2}$ & 0.0135 \\
\hline$-R_{2} \nabla^{2} \psi_{2}$ & 0.0139 \\
\hline$\frac{\partial q_{2}}{\partial t}$ & 0.66
\end{tabular}

Table 6.2: Rms value of terms in lower layer PV equation for a snapshot of the nominal 128 squared hyperviscous vortex solution.

vortex runs indicates that 83 percent is a consistent figure. Apparently small-scale dissipation plays a non-negligible role in energy dissipation of the vortex regime.

\subsection{Comparison to the coherent vortices of earlier studies}

Coherent vortices have been studied extensively in freely decaying two-dimensional turbulence (McWilliams 1984), and in freely decaying equivalent barotropic turbulence (c.f. Larichev and McWilliams 1991 and references therein). McWilliams (1984) noted that it is difficult to find coherent vortices in one-layer forced-dissipated flows. The fronts in the highly viscous f-plane regime of chapter 3 , and the jets in the large $R_{\beta}$ regime of chapter 5 , are coherent structures, but not as remarkable as 


\begin{tabular}{c|c}
\hline \hline Term & Rms Value over Domain \\
\hline \hline$u_{1} \frac{\partial q_{1}}{\partial x}$ & 0.23 \\
\hline$v_{1} \frac{\partial q_{1}}{\partial y}$ & 0.23 \\
\hline$J\left(\psi_{1}, q_{1}\right)$ & 0.08 \\
\hline$\overline{u_{1}} \frac{\partial q_{1}}{\partial x}$ & 0.056 \\
\hline$J\left(\overline{\psi_{1}}, q_{1}\right)$ & 0.056 \\
\hline$v_{1} \frac{\partial \overline{q_{1}}}{\partial y}$ & 0.027 \\
\hline$J\left(\psi_{1}, \overline{q_{1}}\right)$ & 0.027 \\
\hline$-\nu \nabla^{6} q_{1}$ & 0.01 \\
\hline$\frac{\partial q_{1}}{\partial t}$ & 0.085 \\
\hline
\end{tabular}

Table 6.3: Rms value of terms in upper layer PV equation for a snapshot of the hyperviscous 128 squared f-plane solution having the same value of Ekman friction as the nominal 128 squared hyperviscous vortex solution.

the vortices of the current chapter. As discussed earlier, it is possible in a two-layer forced-dissipated system to have strongly nonlinear features such as coherent vortices reside in the layer without Ekman dissipation. Another distinguishing aspect of the present vortices is that they require a nonzero beta to exist, unlike those in McWilliams (1984) and Larichev and McWilliams (1991).

The presence of $R_{d}$ in the equivalent barotropic system slows cascades down considerably compared to those in two-dimensional turbulence. Cascades take place through vortex merger in both cases, but vortex interaction ranges are smaller in the equivalent barotropic case, because of the introduction of the $R_{d}$ scale. The two-layer forced-dissipated problem presented in this chapter also has a deformation radius. Thus the vortices we found here merge slowly when put into a freely 


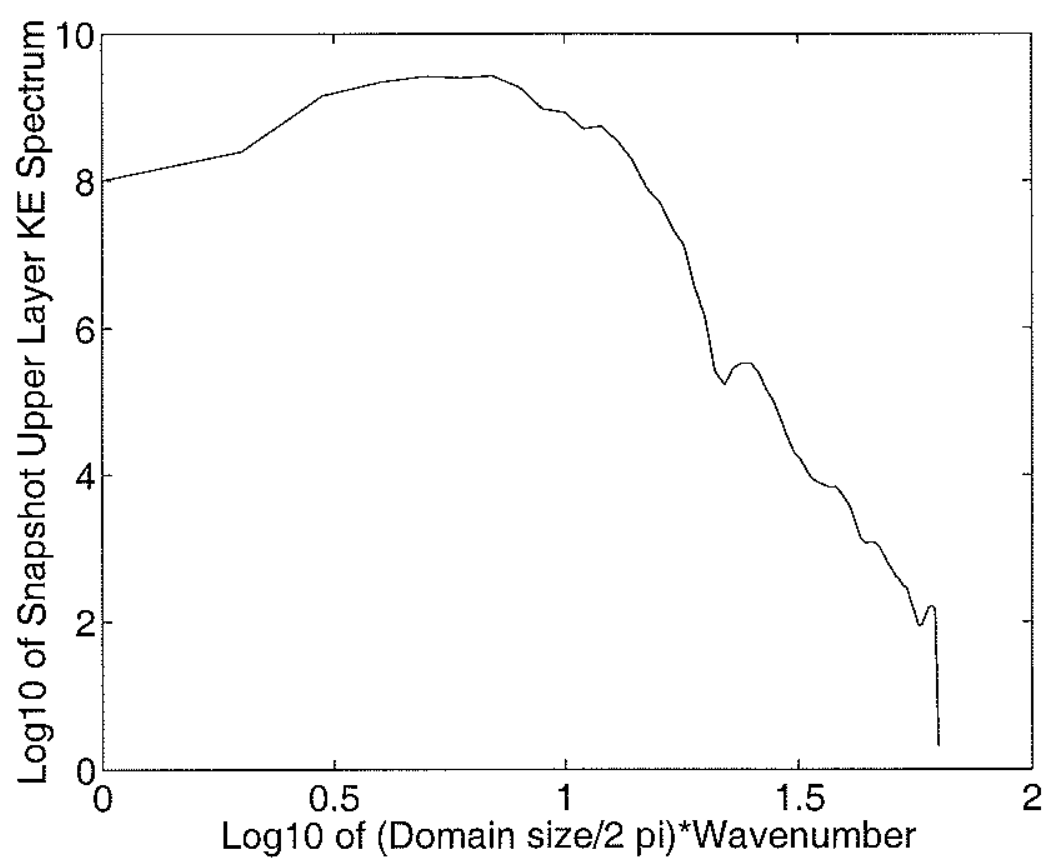

Figure 6-45: Log-log plot of nondimensional upper layer kinetic energy spectrum for snapshot of nominal 128 squared hyperviscous vortex solution. An x-axis value of 1.8 corresponds to a wavelength $\lambda$ equal to $R_{d}$. A value of 1 corresponds to a length scale of $R_{d}$, where length scale is defined as $\frac{\lambda}{2 \pi}$.

decaying problem. The addition of forcing and dissipation can halt the cascade process completely.

A characteristic of the present vortices, as well as of modon/soliton solutions, is a close relationship between streamfunction $\psi$ and potential vorticity $q$ (at least in the upper layer). A close $\psi / q$ relationship is also characteristic of the end state of equivalent barotropic turbulence (figure 6-46), in which $q \sim-\frac{1}{R_{d}^{2}} \psi$ when $L_{e d d y} \gg R_{d}$. The relationship between $\psi$ and $q$ is much less tight in vortices formed in twodimensional turbulence (figure 6-47).

Cyclone/anticyclone asymmetry also distinguishes the vortices presented in this chapter from those in freely evolving two-dimensional and equivalent barotropic turbulence of earlier studies. Cyclone/anticyclone asymmetry does exist in naturefor instance, on Jupiter. Asymmetry is known to exist in freely evolving shallowwater turbulence (c.f. Polvani et al. 1994 and references therein). It was commonly 

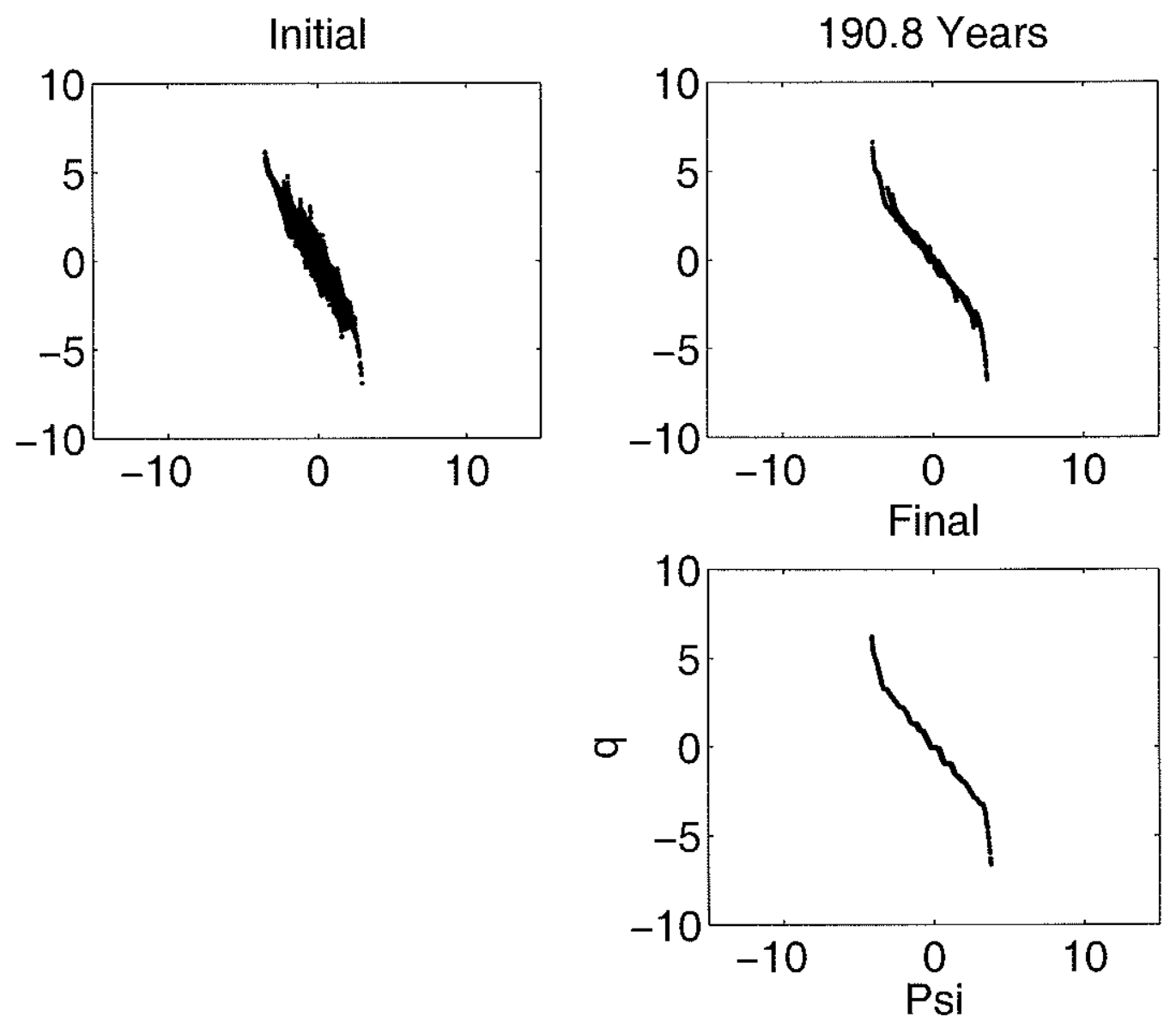

Figure 6-46: Scatter plots of nondimensional PV versus streamfunction for the freely evolving equivalent barotropic solution experiment depicted in figure 6-24. 

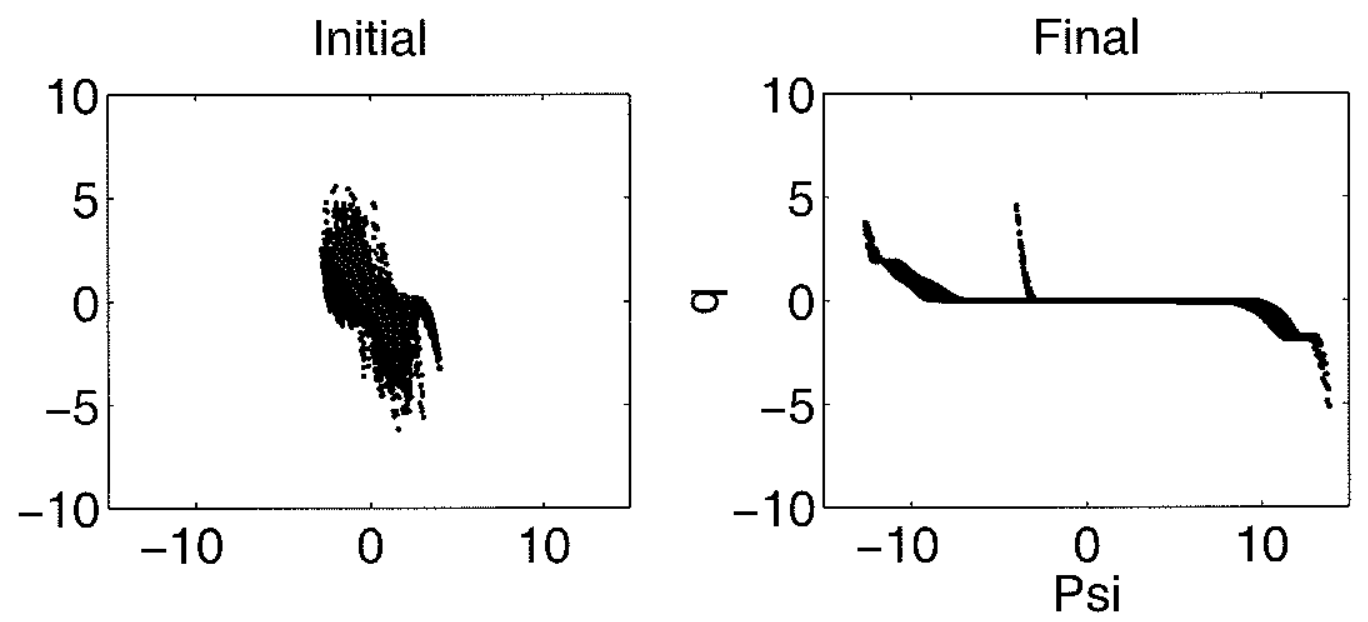

Figure 6-47: Scatter plots of nondimensional PV versus streamfunction for the freely evolving two-dimensional turbulence solution experiment depicted in figure 3-1.

thought not to occur in QG. Now we know that it can. The cause of asymmetry in shallow-water is still not fully explained, long after its discovery (c.f. Kuo and Polvani 2000). QG systems are simpler than shallow-water systems, so there is hope that explaining the cause of the asymmetries in the present experiments will be easier than in the shallow-water case. On the other hand, the present experiments are done on a beta-plane rather than an f-plane, and are forced-dissipated rather than freely evolving. These are complicating factors for the present experiments.

\subsection{Chapter summary}

The vortex regime uncovered in the last chapter was investigated more thoroughly in the present chapter. The first subject discussed was sensitivity to small-scale dissipation. The vortices are more sensitive to small-scale dissipation than are the other solutions presented in this thesis. Under the action of a wavenumber filter, energy increases greatly when resolution increases from 64 squared to 128 squared, so much so that equilibration was not reached in feasible times. In order to obtain 128 squared solutions which equilibrated rapidly, we opted for hyperviscosity as 
our small-scale dissipation in the vortex regime. After some searching, a nominal hyperviscosity was chosen, which was used to generate the 128 squared hyperviscous results presented in this chapter (note: and some of the chapter 5 results also). Hyperviscosity provides some damping at $R_{d}$ scales in the upper layer, which is evidently neccessary for rapid equilibration.

Having chosen a nominal hyperviscosity, we then explored vortex dependence on other parameters. We further discussed dependence on magnitude of $R_{\beta}=$ $\frac{\text { planetary beta }}{\text { upper layer shear gradient }}$ for a fixed magnitude and angle of (westward) shear, which was presented but not much discussed in chapter 5 . It seems that vortices emerge when beta is nonzero but smaller than the magnitude of the upper layer shear-induced PV gradient. However, it was shown that vortices can also be generated from a zonal flow on an f-plane with a topographic beta which is oriented at right angles to the shear gradient. In this case there is no beta in the upper layer to compare the shear gradients with. The topographic case shares with nonzonal flows on a beta plane the fact that layer PV gradients are non-colinear, but that is apparently not the criterion for existence of vortices either, since they were found in purely (westward) zonal flows on a beta plane, in which layer gradients are co-linear. Obviously there is still some confusion as to the criterion for vortex solutions to exist. Vortex behavior was also explored as a function of angle. Vortices can be generated by purely westward shear, as well as purely southward shear-although in the latter case, they exist with a wavenumber filter but not with our nominal hyperviscosity. By symmetry arguments, we know that vortices will also exist for shears that are between westward and northward. So far, no vortex solutions have been found for flows in which the zonal shear is eastward. The governing equations are not invariant under the transformation $\bar{u} \rightarrow-\bar{u}$ because of the presence of $\beta$. Ekman friction was also varied from the value in the nominal experiment. Vortices exist for a wide range of Ekman frictions, although they disappear if the friction term becomes as large as the nonlinear term in the lower layer PV equation. As Ekman friction reduces, 
vortex fields generally gain energy and become more barotropic. However, their length scale does not change, so it is not clear that this is a manifestation of the inverse cascade to the barotropic mode and to larger scales present in the f-plane results of chapter 3 . Vortex size changes very little in the experiments performed in this chapter, despite the variations in beta, angle, friction, and energy encountered. Upper layer length scale, as measured by the reciprocal of the centroid of kinetic energy, is between 1.1 to $1.3 R_{d}$ for all vortex experiments. The Rhines scale does not enter into the problem, contrary to expectations for beta-plane turbulence.

Most of the vortex solutions were generated with $\delta=0.2$, but vortices were also found with $\delta=1$. Their energy is considerably less for the same amount of shear, but comparable when shear is increased so that the upper layer PV gradients are equal. Attempts to generate or sustain vortices with symmetric Ekman friction failed; the vortices seem to prefer to exist in a nearly inviscid layer.

A nonzonal background flow on a beta plane breaks the symmetry between cyclones and anticyclones commonly thought to be ubiquitous in QG. If the nominal solution (cyclones) is inverted and used as an initial condition, the resulting anticyclones equilibrate to a state of lower energy. Various fields were used to initialize the nominal parameters; in all cases, either a high energy state of cyclones or a low energy state of anticyclones emerged. The exact value of the equilibrated energy, as well as the number of vortices, varies; there may be a multiplicity of possible states around these two basic ones. For a flow that is northwestward rather than southwestward, the high energy state is anticyclonic.

Freely decaying experiments initialized by the nominal vortex solution generally undergo an inverse cascade to the barotropic mode and to large horizontal scales, the exception being an experiment in which background PV gradients are set to equal the total mean PV gradients (planetary plus shear-induced) in the nominal forced experiment. The presence of different gradients in the two layers produces enstrophy and holds the inverse cascade at bay. Upper layer vortices disappear 
under the action of bottom Ekman friction, unless there is also a forcing present. This indicates the importance of baroclinic instability in the maintenance of the vortex regime.

Next it was shown that streamfunction and PV have a closer relationship in the vortex solutions than in other solutions presented in this thesis. The dominant balance in both layers is $\frac{\partial q}{\partial t}+J(\psi, q) \approx 0$, unlike, for instance, the f-plane solution having the same value of Ekman friction, in which $J(\bar{\psi}, q)$ and $J(\psi, \bar{q})$ are nearly as large as $J(\psi, q)$. Since forcing enters in at higher order in the vortex regime, structures persist longer than in other regimes. Other solutions have a much less organized $J(\psi, q)$ field, meaning that the Jacobian scatters energy into and out of structures instead of keeping them coherent as in the vortex regime.

In the final section of this chapter differences between the present coherent vortices and those of earlier studies were discussed. The neccessity of nonzero $\beta$, the forced-dissipated nature, and cyclone/anticyclone asymmetry distinguish the present vortices from those previously studied in freely evolving two-dimensional and equivalent barotropic turbulence.

Many unanswered questions remain about the vortex solutions. Their sensitivity to small-scale dissipation requires more explanation. We have indications that the ratio $R_{\beta}=\frac{\text { planetary beta }}{\text { upper layer shear gradient }}$ should be nonzero but less than one for vortices to emerge, but we have not developed a precise criterion. We do not know why they prefer westward shear flows over eastward ones. We know that $R_{d}$ sets the scale of the vortices, but not the reason why. We do not know why the vortex solutions are monopolar. Given that they are, we do know that there should be a symmetry-breaking between states of cyclones and one of anticyclones. Why the asymmetry manifests itself as a difference in energy levels, is unknown. We know that the dominant balance is a modon/soliton type solution, and we also know that small but nonzero beta, friction in the bottom layer only, and baroclinic instability are essential ingredients for producing these vortices. But we have not yet exploited 
these facts to develop a coherent theory for them.

The vortices exist when shear-induced PV gradients are comparable to planetary beta. As discussed in chapter 5, the PV maps of Keffer (1985) indicate that this may be the regime most appropriate for the mid-ocean. Finally, we note that the vortices are strongly baroclinic and at the deformation scale, despite being highly energetic. In this qualitative sense they match the mid-ocean eddy field better than any of the other solutions presented in this dissertation, and are unusually baroclinic and compact for flat-bottom QG solutions. In fact they generally have too much energy and too much baroclinic energy in comparison to observations. 


\section{Chapter 7}

\section{Conclusions}

\subsection{The central question and approach of the dis- sertation}

The central question of this dissertation is whether local baroclinic instability of the weak gyre flows of the ocean interior contributes significantly to the production of mid-ocean eddies. We attempt to answer this question with idealized models of two-layer geostrophic turbulence, forced by an imposed, horizontally homogeneous, vertically sheared mean flow, and dissipated through Ekman friction. The dissertation focuses on processes that control the following characteristics of the mid-ocean eddy field: 1) eddy kinetic energy is roughly equipartitioned in the vertical between the barotropic and first baroclinic modes (c.f. Wunsch 1997), 2) horizontal length scales of the eddies appear to correlate better with the first baroclinic mode deformation radius $\left(R_{d}\right)$ than with larger cascade scales (Stammer 1997), and 3) eddy kinetic energy is much larger than kinetic energy of the mean flow (c.f. Gill et al. 1974).

Linear baroclinic instability theory predicts that fluctuation length scales will be near $R_{d}$, and that substantial energy will be in the baroclinic mode. However, 
Rhines (1977) demonstrated that nonlinear effects (which will be important when the ratio of eddy to mean kinetic energy is larger than one) shift eddy energy into the barotropic mode and into large horizontal scales, in freely-evolving turbulence. Salmon $(1978 ; 1980)$ and Larichev and Held (1995) found similar tendencies in two-layer forced-dissipated turbulence--but all three of these studies had equal layer depths, which is representative of a uniform stratification. We expect two-layer models with a thin upper layer, which represents a surface-trapped, thermoclinelike stratification, will have enhanced eddy baroclinicity (and, associated with that, horizontal scales near $R_{d}$ ) for two reasons. The first is that nonlinear interactions within the baroclinic mode project onto the baroclinic mode if and only if layer depths are unequal. The second is that the baroclinic mode is weakly damped if stratification is surface-trapped and friction occurs at the bottom. In chapters 3 and 4, with these considerations in mind, we perform and examine four sets of forceddissipated runs on an f-plane. Two of them have Ekman friction in the bottom layer only and are contrasted in their values of the parameter $\delta=\frac{H_{1}}{H_{2}}$. One bottom-friction set has $\delta=0.2$ (surface-trapped stratification), while the other has $\delta=1$ (uniform stratification). Two other sets of forced-dissipated runs were run with the same values of $\delta$ but with equal Ekman damping rates in the two layers, which we refer to as symmetric friction. Surface-trapped stratification enhances eddy baroclinicity only through the nonlinear projection mechanism in this case, which therefore serves as an important contrasting example.

On the f-plane, arbitrarily small shears are baroclinically unstable. However, weak zonal shears are stable in the presence of planetary beta, which would seem to indicate that it is difficult to produce eddies from the weak mean flows typical of the mid-ocean. It has been known for some time, from linear instability theory, that nonzonal flows are unstable even in the presence of $\beta$, and the presence of continental boundaries forces mid-ocean mean flows to be nonzonal. In chapter 5 , a nonlinear equilibration study is performed to ascertain whether the nonzonality 
inherent in ocean gyres could explain the fact that mid-ocean eddy kinetic energies are much larger than the mean flow kinetic energy, despite the fact that mid-ocean mean flows are weak and planetary beta is present. Energy is studied as a function of angle for a fixed magnitude of planetary beta and weak mean shear, such that $R_{\beta}=\frac{\text { planetary beta }}{\text { upper layer shear gradient }}=6$. In this case the zonal experiment does not meet the Charney-Stern criterion for baroclinic instability. A second set of experiments is performed in which shear angle is held fixed but $\beta$ (thus $R_{\beta}$ ) is varied. This set uncovered a vortex regime which is investigated extensively in chapter 6 .

\subsection{Major results of the dissertation}

In chapter 3 , it is found that, for a fixed amount of total eddy energy, the symmetric friction $\delta=0.2$ experiments generally retain slightly more kinetic energy in the baroclinic mode than do the $\delta=1$ experiments. The offset is substantially larger between $\delta=0.2$ and $\delta=1$ experiments with bottom friction, implying that the weak damping of the baroclinic mode exerts a stronger control on eddy baroclinicity then does the nonlinear projection mechanism. In bottom friction experiments, two regimes exist, delineated by the value of $\frac{\delta U_{1} L_{2}}{(1+\delta) R_{2} R_{d}^{2}}$, which scaling suggests is the approximate ratio of nonlinear to friction terms in the lower layer PV equation. (In this expression, $U_{1}$ is the upper layer rms eddy velocity, $L_{2}$ is the lower layer eddy scale, and $R_{2}$ is the coefficient of bottom Ekman friction). When friction dominates, bottom layer velocities go to zero, which implies the relationship $\frac{K E_{B C}}{K E_{B T}}=\frac{H_{2}}{H_{1}}$. When nonlinear terms are larger, eddies barotropize as in previous studies. Thus, $\frac{H_{2}}{H_{1}}$ is an upper bound on the baroclinicity ratio for bottom friction experiments, meaning that it is possible for the baroclinic mode to retain more kinetic energy than the barotropic mode when stratification is surface-trapped, bot not when stratification is uniform. Mid-ocean current meter data indicates that the baroclinic mode generally has somewhat more kinetic energy than does the barotropic mode. Therefore, 
the surface-trapped nature of oceanic stratification, and the bottom-trapped nature of the dissipation, may exert a strong control on the partition of kinetic energy between modes. The $\delta=0.2$ bottom friction experiments with 145, 193, and 290 day dissipation times have the most realistic ratios of eddy to mean kinetic energy (21, 27 and 49 , respectively). The baroclinicity ratios $\frac{K E_{B C}}{K E_{B T}}$ for these are 1.5, 1.1, and 0.7 , respectively. The first of these compares well with the meter data of Wunsch (1997), while the last is not quite baroclinic enough. Top layer length scales of these experiments, measured as $\frac{1}{2 \pi}$ of the wavelength of maximum kinetic energy, vary from about $1.5 R_{d}$ to $2 R_{d}$, which compares well with the Stammer (1997) altimeter estimates. When measured as inverses of the centroids of kinetic energy, top layer eddy length scales for these experiments range from $1.1 R_{d}$ to $1.3 R_{d}$. Thus, the $\delta=$ 0.2 bottom friction f-plane experiments having the most realistic ratios of eddy to mean kinetic energy have length scales and baroclinicity ratios that compare reasonably well with data. In all four sets of experiments, when the ratio of lower layer nonlinear to Ekman friction terms exceeds one, the barotropic length scale cascades far beyond the baroclinic kinetic energy scale, which remains near $R_{d}$, as anticipated from the association of the baroclinic mode with the deformation radius. When $\delta=0.2$, top layer kinetic energy is dominated by the baroclinic mode while the barotropic mode is more important in the lower layer. Hence the top layer eddy scale is smaller than that of the bottom layer in the $\delta=0.2$ experiments, which is consistent with the findings of Mercier and de Verdiere (1985). Finally, we note that a cutoff value of friction exists in the symmetric friction case; an equilibrated eddy field does not exist as friction is increased beyond this cutoff value. Near the stability boundary the symmetric friction experiments exist in a state of weakly nonlinear waves. As friction reduces, the waves become more strongly nonlinear before finally breaking up into turbulence. There is no stability boundary for the bottom friction experiments, which never appear wavelike.

In chapter 4, a cascade inequality is developed from the energy and enstrophy 
equations. The inequality takes on a simple form in the case of symmetric Ekman friction:

$$
1 \geq R_{d}^{2} \frac{\left[k_{B T}^{2} K E_{B T}+k_{B C}^{2} K E_{B C}\right]}{\left[K E_{B T}\right]}
$$

where brackets denote the time-averaging operator and the wavenumbers $k_{B T}$ and $k_{B C}$ are centroids of modal kinetic energy spectra. If correlations within brackets are ignored, the inequality simplifies to:

$$
1 \geq R_{d}^{2} k_{B T}^{2}+R_{d}^{2} k_{B C}^{2} \frac{K E_{B C}}{K E_{B T}}
$$

which means that in the symmetric friction case, it is impossible to simultaneously retain substantial energy in the baroclinic mode and in length scales near $R_{d}$. On the other hand, the right-hand side of 7.1 exceeds one in $\delta=1$ bottom friction experiments, and greatly exceeds one in $\delta=0.2$ bottom friction experiments. Thus, the surface-trapped nature of ocean stratification and the bottom-trapped nature of the dissipation appear to be crucial factors in allowing eddies to simultaneously retain substantial energy in the baroclinic mode and in scales near $R_{d}$, as happens in the actual ocean.

Chapter 5 shows that when $R_{\beta}=6$, energy is indeed a strong function of angle. This suggests that those parts of the mid-ocean having mean flows which are nearly meridional may generate the bulk of the mid-ocean eddy energy. The values of eddy energy are actually far too large when compared to the actual ocean. The meridional flow experiment has a ratio of eddy to mean kinetic energy exceeding 7000 , while this ratio for the 195 degree experiment (mean shear 15 degrees south of west) is 81 . Thus it certainly is possible in these idealized experiments to generate eddy kinetic energies much larger than the mean even for experiments in which $\beta$ dominates the PV gradients. The angle between layer PV gradients is small in these 
experiments, which are strongly anisotropic, much more so than is the actual midocean eddy field. A set of experiments with fixed angle and magnitude of shear, but varying beta, and hence varying $R_{\beta}$, was then conducted. When $R_{\beta}$ is less than one, the angle between layer PV gradients is large, as the Keffer (1985) maps of oceanic potential vorticity suggest may be the case in the mid-ocean, and eddy fields become isotropic. If, in addition, the zonal component of the mean shear is westward, vortex solutions emerge.

The vortex regime is explored further in chapter 6 . Vortices emerge for a wide variety of shear angles and values of Ekman friction. As with the large $R_{\beta}$ experiments, the vortex experiments are extremely energetic. Vortex experiments with dissipation times of 48.4 and 774 days, respectively, each have top layer length scales of $1.3 R_{d}$, while top layer kinetic energies are 29000 and 1400 times the mean kinetic energy, and baroclinicity ratios are 4.2 and 1.3. In a sense, these solutions meet our three chosen observations of interest too well; they have too much energy and too much baroclinic energy. The vortex solutions are monopolar and exhibit an asymmetry in energy levels between cyclones and anticylones which is probably unrealistic for the actual ocean. Compared to the other results in this thesis as well as those in the existing literature, they exhibit a remarkable degree of coherence considering that they exist in a forced-dissipated system. Thus they might serve as a useful model for maintenance of coherent structures, such as Jupiter's Great Red Spot, Gulf Stream Rings, atmospheric blocking events, and oceanic submesoscale coherent vortices (c.f. Flierl 1987, McWilliams 1985) in forced-dissipated geophysical flows.

\subsection{New regimes discovered in the dissertation}

Three interesting regimes of geostrophic turbulence were uncovered in the course of answering our original questions: 
1) When the bottom Ekman friction term is larger than the nonlinear term in the lower layer PV equation, a regime exists whose appearance is quite unlike that seen in the "standard" f-plane geostrophic turbulence literature (c.f. Larichev and Held 1995). Some quantities in this regime, for example, the ratio of baroclinic to barotropic kinetic energies, and the fraction of enstrophy dissipation accounted for by Ekman friction, can be quantitatively explained from simple arguments. Other correlations exist in this regime which the author has not yet explained. The nonmonotonic behavior of energy with friction, and the existence of fronts in this regime have not been explained yet either; some speculations were offered in chapter 3 .

2) Eddy fields forced by nonzonal flows on a beta plane in which beta is much larger than the shear-induced PV gradients are generally dominated by a single zonal jet which slowly drifts throughout the domain. Jet width is narrow and apparently unrelated to the Rhines length. We speculate that spatial inhomogeneities develop in this regime in order to bring about divergences of eddy fluxes. These can serve as an equilibrating mechanism for the jets, which would be difficult to equilibrate otherwise, since they are able to extract energy from the meridional component of the nonzonal mean flow.

3) A coherent vortex regime was explored in detail in chapter 6 . Now that the parameter space in which they exist has been delineated, fundamental questions about their dynamics are just beginning to be explored.

\subsection{Rescaling the equations for different values of friction, shear, and beta}

A general problem with our numerical results with regard to comparison to the actual ocean is that they depend strongly on the Ekman dissipation time. We have chosen to do experiments with a fixed $1 \mathrm{~cm} \mathrm{~s}^{-1}$ mean shear flow and a fixed $R_{d}=$ $50 \mathrm{~km}$; for these parameters the $\delta=0.2 \mathrm{f}$-plane bottom friction experiments with 
dissipation times in the range 145 to 290 days have the most realistic ratios of eddy to mean kinetic energy. As discussed in chapter 3, however, estimates of dissipation times vary widely; all we can say is that 145 to 290 days is not too far out of the range of most estimates. High resolution results were obtained with only one value of friction in the large $R_{\beta}$ jet regime of chapter 5 . However, 64 squared runs performed in this regime (not included in the thesis) also showed a sensitivity to friction. The vortex results of chapter 6 depend on Ekman friction as well.

As discussed in chapter 2 , our chosen values of mean shear and $R_{d}$ seem reasonable. Our choice to do experiments with a fixed value of shear throughout the thesis was done for pragmatic reasons, as the amount of parameter space we must study is already quite large. Since values of mean shear are not very well known, however, this choice might seem overly restrictive. Another point is that for our choice of mean shear, the values of $\beta$ that give layer PV gradients in very different directions, as indicated in the PV maps of Keffer (1985), are much smaller than is realistic. We ask now whether the governing equations can be rescaled to cover the case of larger beta and larger shear. The governing lower layer equation having hyperviscosity as a small-scale dissipation is used to illustrate the point (the argument with the upper layer equation works the same way):

$$
\begin{gathered}
\frac{\partial q_{2}}{\partial t}+\overline{u_{2}} \frac{\partial q_{2}}{\partial x}+ \\
+\overline{v_{2}} \frac{\partial q_{2}}{\partial y}-\frac{\delta\left(\overline{v_{1}}-\overline{v_{2}}\right)}{(1+\delta) R_{d}^{2}} \frac{\partial \psi_{2}}{\partial y}+\left(\beta+\frac{\delta\left(\overline{u_{2}}-\overline{u_{1}}\right)}{(1+\delta) R_{d}^{2}}\right) \frac{\partial \psi_{2}}{\partial x} \\
+J\left(\psi_{2}, q_{2}\right)=-R_{2} \nabla^{2} \psi_{2}+\nu \nabla^{6} q_{2} .
\end{gathered}
$$

There are four terms in the equation involving $\beta$ or a mean velocity term multiplied by derivative operators acting on $\psi_{2}$ or $q_{2}$. There are two terms involving an Ekman or hyperviscous dissipation multiplied by derivative operators acting on $\psi_{2}$ or $q_{2}$. Supposing that the mean velocities, planetary beta, $R_{2}$, and $\nu$ were all multiplied by the same factor, call it $\alpha$. Then, if the nonlinear term were not present, the 
equation would be invariant if the time variable were rescaled, so that everything would happen $\alpha$ times faster. Because of the Jacobian term, the fluctuation streamfunctions (and, as a consequence, potential vorticities) must also be multiplied by $\alpha$ to make equation 7.2 invariant. Thus, if $\beta, \overline{u_{1}}, \overline{u_{2}}, \overline{v_{1}}, \overline{v_{2}}, \psi_{1}, \psi_{2}, R_{2}$, and $\nu$ are all increased by $\alpha$, the equation is invariant under a time scale which speeds up by the same factor $\alpha$. Suppose, for instance, that in our f-plane experiments we increased our mean shear to $4 \mathrm{~cm} \mathrm{~s}^{-1}$, a value used in Gill et al. (1974). Then the most realistic ratios of eddy to mean kinetic energy would occur for experiments having Ekman spin-down times of 36 to 72 days, rather than 145 to 290 days. (Note, though, that values of eddy kinetic energy would be 16 times larger, far too large when compared to observations.) Even stronger frictions, much larger than indicated by observations, would be required in order to bring about realistic eddy kinetic energies if we were to multiply the mean by 4 . Suppose instead that we wanted to know if layer PV gradients can be at large angles to each other for realistically large values of $\beta$. For $\beta=2 \bullet 10^{-11} \mathrm{~m}^{-1} \mathrm{~s}^{-1}$, shear values of $8 \mathrm{~cm} \mathrm{~s}^{-1}$ would create an angle larger than 90 degrees, which would lead to an isotropic eddy field, and to isotropic vortices if the zonal component of shear is westward. This is too large a mean velocity for the mid-ocean. More likely, as discussed earlier, is that the nominal values of shear and beta $\left(1 \mathrm{~cm} \mathrm{~s}^{-1}, 2 \bullet 10^{-11} \mathrm{~m}^{-1} \mathrm{~s}^{-1}\right)$ we have chosen are realistic, and the reason that PV gradients can turn so much with depth is that in the actual ocean, a great deal of shear occurs over the depth of the thermocline. This is not possible to model in two layers, which represent the thermocline by a single gradient vector.

\subsection{Limitations of homogeneous turbulence mod- els as a representation of the mid-ocean}

Ocean basins typically contain several tens of eddy length scales. The mid-ocean has therefore been considered a region in which homogeneous turbulence models might 
be applicable (c.f. Larichev and Held 1995). Having adopted the same viewpoint in this dissertation, we now discuss some of the drawbacks of homogeneous turbulence models as a representation of the mid-ocean.

\subsubsection{Eddy feedbacks onto the mean}

The actual mid-ocean mean flow is not horizontally homogeneous, meaning that there will be divergences of eddy PV fluxes which in turn alter the mean flow. This is an important mechanism for equilibration in weakly nonlinear channel models of baroclinic instability (c.f. Pedlosky 1970), and it has long been known that eddy flux divergences are important in the maintenace of the general atmospheric circulation (c.f. Peixoto and Oort 1992). This mechanism was neglected in the formation of the model used in this thesis. Our solutions equilibrate through bottom Ekman friction (although, interestingly, flux divergences do arise spontaneously and contribute to equilibration in the jet solutions of chapter 5). We judge the relative importance of eddy flux divergences and bottom friction as equilibration mechanisms by estimating spin-down timescales for the two processes.

The zonal mean PV of a zonal mean flow is altered by eddy flux divergences through the following equation (c.f. Pedlosky 1987):

$$
\frac{d \bar{q}}{d t}=-\frac{d}{d y} \overline{v q}
$$

where overbars denote a zonal average. Suppose that eddies equilibrate through bringing the mean PV gradient $\frac{d \bar{q}}{d y}$ to zero, as in the baroclinic adjustment hypothesis of Stone (1978); this would occur over a time:

$$
T_{\text {feedback }} \sim \frac{L_{\text {mean }} \frac{d \bar{q}}{d y}}{\frac{d \bar{q}}{d t}}=\frac{L_{\text {mean }} \frac{d \bar{q}}{d y}}{\frac{d}{d y} \overline{v q}},
$$

where $L_{\text {mean }}$ is the scale over which the mean flows vary. If we scale $\frac{d}{d y}$ as $\frac{1}{L_{\text {mean }}}$ and $q$ as $L_{e d d y} \frac{d \bar{q}}{d y}$, we obtain: 


$$
T_{\text {feedback }} \sim \frac{L_{\text {mean }}^{2}}{v L_{\text {eddy }}} .
$$

If we take $L_{\text {mean }} \sim 1000 \mathrm{~km}$ (a patch of the gyre, rather than the entire gyre), $v \sim$ $10 \mathrm{~cm} \mathrm{~s}^{-1}$, and $L_{e d d y} \sim 100 \mathrm{~km}$, we obtain $T_{\text {feedback }} \sim 1000$ days. Note that a similar scaling could be applied to erase the zonal gradients $\frac{d \bar{q}}{d x}$. In that case the relevant PV flux would be $\frac{d}{d x} \overline{u q}$. Following Spall (2000), in that case we might write $q \sim L_{\text {mean }} \frac{d \bar{q}}{d x}$,

leaving $T_{\text {feedback }} \sim \frac{L_{\text {mean }}}{v} \sim 100$ days, less by a factor of $\frac{L_{\text {mean }}}{L_{\text {eddy }}}$. If $L_{\text {mean }}$ were instead taken to be a typical basin length, say, $4000 \mathrm{~km}$, the feedback times increase to 16000 and 400 days. The zonal gradients therefore could be erased in 100-400 days, while approximately 1000-16000 days are required to erase the meridional gradients. Frictional spin-down times are not well known-recall the discussion in chapter 3 . Some estimates are that they are substantially less than 1000 days. If so, eddies would spin down by friction before they have a significant effect on mean flows, and friction would be the more important equilibration mechanism. If the spin down times are longer, the two equilibration mechanisms might be of similar importance.

\subsubsection{Finite domain size}

The assumption one makes in using homogeneous turbulence models is that they contain many eddy lengths. Related to this, one assumes that domain size does not affect the dynamics under examination. This was not explicitly examined in this thesis, but should be the case as long as eddy lengths are not comparable to domain size, Length scales of total eddy energy are comparable to domain size for both the large friction and small friction ends of the bottom friction only experiments of chapter 3 . The same holds for the chapter 3 symmetric friction experiments. These limiting cases helped us to probe and understand the behavior of the whole range of forced-dissipated f-plane turbulence. However, the experiments which were held up as being most representative of the mid-ocean had length scales substantially less 
than domain size. Thus we would not expect domain size to play a role in the most realistic f-plane experiments. Experiments (not reported in this document) indicate that domain size plays no role in the vortex regime of chapter 6 . Preliminary results indicate that the number of jets in the large $R_{\beta}$ regime of chapter 5 may depend on domain size, but energy levels do not.

\subsubsection{Doubly periodic boundary conditions}

The doubly periodic boundary conditions used in this thesis undoubtedly have an effect on the large $R_{\beta}$ jet regime of chapter 5 . It is to be hoped that the main conclusion of that set of experiments-that eddy energy depends strongly on the angle of the mean shear flow when $R_{\beta}$ is much greater than one-would hold even in a bounded domain. Spall (2000) demonstrates that this is probably the case. But it seems unlikely that strong jets would exist in a bounded domain. None were seen in the Spall (2000) calculations.

\subsubsection{Spinup time}

Many of the experiments in this dissertation take a long time to spin up to equilibrium-

for instance, the $R_{\beta}=12$ jet and $R_{\beta}=0.375$ vortex experiments of chapter 5 both took about 500 years to equilibrate. While winds have been blowing over the ocean for much longer than that, they obviously vary a great deal on shorter time scales. The eddy field in the actual ocean does not have the space nor the time that we have allowed for our homogeneous model to develop. Thus, one suspects that the energies developed in the present experiments may be too high. In fact many of our experiments have too much eddy energy, when compared to observations, and this may be part of the reason. 


\subsection{Plausibility of the local baroclinic instability mechanism}

It is possible for homogeneous baroclinically unstable flows to produce eddies for which the ratio of eddy to mean kinetic energy is much larger than one, for which the baroclinic mode has slightly more kinetic energy than the barotropic mode, and for which the eddy length scale is near $R_{d}$. It is difficult to meet all of these criterion simultaneously, in an environment which is realistic. Some of the $\delta=0.2$ f-plane results from chapter 3 satisfy the three conditions reasonably well, and, in addition, are isotropic, which is also in accordance with observations. However, the f-plane model ignores planetary beta, which stabilizes weak zonal flows. Eddy kinetic energy much larger than the mean can be generated from nonzonal flows even when $\beta$ is present and larger than shear-induced PV gradients, but the eddies then become more anisotropic (and more energetic) than in observations. The vortex regime of chapter 6 exists when the layer PV gradients are at large angles to each other, as suggested in the maps of Keffer (1985). Vortex solutions are isotropic, and retain substantial energy in the baroclinic mode and in $R_{d}$ scales, but they have an unrealistic monopolar nature and exist only when the mean shear has a westward zonal component. Some disagreement with observations can be found in all of our model solutions. However, we have shown that it is possible for eddy fields to extract energy from the mean, even with beta present, and we have produced some explanations for the factors that control the energy, baroclinicity, length scales, and isotropy of the mid-ocean eddy field. On the whole, local baroclinic instability seems to be a plausible mechanism for mid-ocean eddy generation.

\subsection{Future work}

Logical extensions to the current thesis work include: 
1) Incorporation of more realistic bottom friction schemes into the model. As discussed in chapter 3, work on bottom boundary layers suggests a quadratic law for dissipation rather than the linear law used here. It is likely that generation and breaking of internal waves over rough topography is also a major dissipative mechanism in the ocean. Both occur at the bottom, so our belief is that the baroclinic mode will still be weakly damped under these circumstances. Analytical work (for instance, development of a cascade inequality) would be more difficult with these more realistic dissipations than with Ekman friction. Parameterizing the effects of internal wave breaking is a topic of current research.

2) Incorporation of more realistic stratification-i.e., more layers. The second baroclinic mode, absent in the present study, typically contains about ten percent of the water-column averaged kinetic energy (Wunsch 1997). The most energetic fplane results from chapter 3 are still too barotropic and too large, in comparison to observations. The results of Fu and Flierl (1980) and Smith and Vallis (2000) demonstrate that higher baroclinic modes transfer energy to the first baroclinic mode, which should render the first mode more energetic. A multi-layer version of equation 3.30 should place an upper bound on the sum of all baroclinic modal kinetic energies, as a function of stratification. Retention of substantial energy in the second baroclinic mode, which has a smaller deformation radius, should make it possible for model eddy fields to remain near $R_{d}$ over a larger range of Ekman frictions. A multi-layer version of the cascade inequality would help to quantify the effects of higher modes in this process. It would also be interesting to see if coherent vortices would show up in the higher modes of a multi-layer model run in the parameter regime of chapter 6 . If so, perhaps this would be related to the submesoscale coherent vortices of McWilliams (1985), which are too small to be first baroclinic mode features.

3) Incorporation of topography-both slope and roughness-into the model. The cascade inequality developed in this thesis can be extended to include topography. 
Effects of topography on the inverse cascade in a forced-dissipated system can then be quantified. Preliminary results suggest that sign-indefinite terms introduced by topography do indeed act to prevent the cascade, meaning that model results may remain baroclinic and compact over a wider range of friction values. However, as we saw in chapter 4 , sign-indefinite terms play a role that, while important, is secondary. What is more important is that, when friction is bottom-trapped, the sign-definite baroclinic terms are multiplied by the factor $\frac{H_{1}}{H_{2}}$. With topography present, the cascade inequality still points towards bottom-trapped friction coupled with surface-trapped stratification as the primary factor in producing an eddy field that is baroclinic and near $R_{d}$ at the same time. Thus, these factors will still affect eddy characteristics even with topography present.

4) Extension to shallow-water systems. Quasi-geostrophy $(\mathrm{QG})$ is often used because of its relative simplicity. Shallow-water (SW) models are closer to the true NavierStokes equations, however, and retention of extra physics in one-layer SW turbulence has profound effects (c.f. Polvani et al. 1994). For instance, SW turbulence collects at scales comparable to the deformation radius. This is unlike the behavior of purely two-dimensional turbulence, which cascades to larger scales. Two-dimensional turbulence does not contain any inherent length scales. The deformation scale can enter into one-layer QG dynamics, in the equivalent barotropic system studied briefly in chapter 6 . We showed there that the inverse cascade proceeds in this system, albeit slowly. Our theoretical argument points towards the first moment of the spectrum of total eddy energy, rather than kinetic energy only, as the scale that will undergo the cascade. What we have shown therefore is that the presence of a deformation radius is not sufficient to halt the inverse cascade in one-layer QG. Why, then, is the cascade arrested in one-layer SW turbulence? It may have to do with geostrophic adjustment at the deformation radius in shallow-water systems (Kuo and Polvani 2000). Another possible explanation lies in the SW mass conservation equation, which in the nondimensional form used by Polvani et al. 1994 is: 


$$
R o *\left(\frac{L}{R_{d}}\right)^{2}\left[\frac{\partial h}{\partial t}+\nabla \bullet(h \vec{u})\right]+\nabla \bullet \vec{u}=0
$$

where Ro is the Rossby number (typically small but not negligibly so in mid-ocean eddy flows), $\mathrm{L}$ is the length scale of the eddies, $\mathrm{h}$ is the nondimensional perturbation height, and $\vec{u}$ is the horizontal velocity. In QG models, order Rossby number terms are dropped, leaving $\nabla \bullet u=0$ as the mass conservation equation. QG is inherently two-dimensional. Velocities are derivatives of a streamfunction, which allowed us to make arguments analogous to Fjortoft's in the equivalent barotropic case (and in the two-layer case, back in chapter 3). On the other hand, in SW, when $L \sim R_{d}$ and the Rossby number is nonzero, $\nabla \bullet u=0$ is no longer satisfied. Shallow-water turbulence is therefore inherently less two-dimensional at the deformation scale. Total energy and potential enstrophy are still conserved in shallow-water turbulence:

$$
\begin{gathered}
\frac{\partial}{\partial t} \iint \frac{1}{2} h \vec{u} \bullet \vec{u} d x d y=\frac{\partial}{\partial t} \int E(k) d k=0, \\
\frac{\partial}{\partial t} \iint \frac{1}{2} h\left(\frac{f+\frac{\partial v}{\partial x}-\frac{\partial u}{\partial y}}{h}\right)^{2} d x d y=\frac{\partial}{\partial t} \int Q(k) d k=0 .
\end{gathered}
$$

However, $\mathrm{Q}(\mathrm{k})$ does not equal $k^{2} E(k)$ (at least as far as the author can tell!), and the familiar cascade arguments cannot be applied. A one-layer SW model initialized with the same flow fields applied to the equivalent barotropic system in chapter 6 is therefore anticipated not to undergo a cascade as vigorous as in the equivalent barotropic case. (Note that Polvani et al. 1994 used the first moment of kinetic energy as a measure of length scale. To compare to the present equivalent barotropic results we would have to use the scale of total energy as our measure. Also, we would need to integrate for comparable times as in the equivalent barotropic case.) Supposing that were successful, the next logical step would be to extend the results 
to two layers. As described in chapter 3, part of the inverse cascade in freely evolving two-layer QG flows is an energy transfer from baroclinic to barotropic modes. In a two-layer shallow water system, the baroclinic mode mass conservation equation would likely resemble 7.6, meaning that we would expect the fluid to stop behaving two-dimensionally at that scale. Two-layer SW models are therefore anticipated not to undergo the barotropization inherent in QG models. If this were true, it would have two important consequences. First, we would have discoverered a freely evolving fluid which retains its vertical structure and horizontal compactness, in accordance with observations. This would complement the work done in this dissertation, which required forcing and dissipation to keep a two-layer QG fluid baroclinic and near $R_{d}$. Although the ocean is forced and dissipated, both are weak, so there would be value in finding a freely evolving fluid that better matched the observations. Secondly, as we have seen here, freely evolving models can help explain the behavior of forced-dissipated systems, in a qualitative way. Just as the long-time limit of freely evolving QG tends towards a state of large, barotropic eddies, so does the forced-dissipated problem, when friction becomes very small. If indeed the tendency towards barotropization and the inverse cascade is reduced in freely-evolving two-layer SW, that would likely indicate less sensitivity to the exact value of friction used in the forced-dissipated problem. In the present work, the forced results with small values of Ekman friction are still too large and too barotropic. Based on the above reasoning, the author expects forced-dissipated SW systems to behave differently. 


\section{References}

Barcilon, V., 1964: Role of the Ekman layers in the stability of the symmetric regime obtained in a rotating annulus. J. Atmos. Sci., 21, 291-299.

Batchelor, G.K., 1953: The theory of homogeneous turbulence. Cambridge University Press, Cambridge, 121 pp.

Batchelor, G.K., 1969: Computation of the energy spectrum in homogeneous twodimensional turbulence. Phys. Fluids Suppl., 12, II 233-239.

Bretherton, F.B., 1965: Baroclinic instability and the short wavelength cut-off in terms of potential vorticity. Quart. J. Royal Met. Soc., 92, 335-345.

Bretherton, F.B., and D.B. Haidvogel, 1976: Two-dimensional turbulence over topography. J. Fluid Mech., 78, 129-154.

Bryden, H.L., 1979: Poleward heat flux and conversion of available potential energy in Drake Passage. J. Marine Res., 37, 1-22.

Bryden, H.L., 1982: Sources of eddy energy in the Gulf Stream recirculation region. J. Marine Res., 40, 1047-1068.

Canuto, C., M.Y. Hussaini, A. Quarteroni, and T.A. Zang, 1988: Spectral methods in fluid mechanics. Springer-Verlag, 567 pp.

Charney, J.G., 1947: The dynamics of long waves in a baroclinic westerly current. J. Meteorol., 4, 135-163.

Charney, J.G., and M.E. Stern, 1962: On the stability of internal baroclinic jets in a rotating atmosphere. J. Atmos. Sci., 19, 159-172.

Charney, J.G., 1971: Geostrophic turbulence. J. Atmos. Sci., 28, 1087-1095.

Cheney, R.E., and P.L. Richardson, 1976: Observed decay of a cyclonic Gulf Stream ring. Deep-Sea Res., 23, 143-155.

Chester, D., P. Malanotte-Rizzoli, J. Lynch and C. Wunsch, 1994: The eddy radiation field of the Gulf Stream as measured by ocean acoustic tomography. Geophys. Res. Lett., 21, 181-184.

Cushman-Roisin, B., 1994: Introduction to geophysical fluid dynamics, Prentice Hall, New Jersey, 320 pp. 
Dubus, L., 1999: Baroclinic instability of the northeast Atlantic mid-latitude meridional currents; impacts on the large scale circulation and associated tracer mixing. PhD dissertation, Universit de Bretagne Occidentale, Brest, France.

Eady, E.T., 1949: Long waves and cyclone waves. Tellus, 1, 33-52.

Fjortoft, R., 1953: On the changes in the spectral distributions of kinetic energy for two-dimensional non-divergent flow. Tellus, 5, 225-230.

Flierl, G.R., 1977: The application of linear quasigeostrophic dynamics to Gulf Stream rings. J. Phys. Oceanogr., 7, 365-379.

Flierl, G.R., 1978: Models of vertical structure and the calibration of two-layer models. Dyn. Atmos. Oceans, 2, 341-381.

Flierl, G.R., 1987: Isolated eddy models in geophysics. Ann. Rev. Fluid Mech., 19, 493-530.

Flierl, G.R., V.D. Larichev, J.C. McWilliams, and G.M. Reznik, 1980: The dynamics of baroclinic and barotropic solitary eddies. Dyn. Atmos. Oceans, 5, $1-41$.

Flierl, G.R., and V.M. Kamenkovich, 1975: Gulf Stream meandering and Gulf Stream ring eddy production mechanisms. Pages 115-118 of: Robinson, A.R. (ed), Dynamics and the analysis of MODE-I. MIT.

Frankignoul, C., and P. Muller, 1979: Quasi-geostrophic response of an infinite beta-plane ocean to stochastic forcing by the atmosphere. J. Phys. Oceanogr., 9, 104-127.

Fu, L.-L., and G.R. Flierl, 1980: Nonlinear energy and enstrophy transfers in a realistically stratified ocean. Dyn. Atmos. Oceans, 4, 219-246.

Fu, L.-L., T. Keffer, P. Niiler, and C. Wunsch, 1982: Observations of mesoscale variability in the western North Atlantic: A comparative study. J. Marine Res., 40, 809-848.

Fuglister, F.C., and L.V. Worthington, 1951: Some results of a multiple ship survey of the Gulf Stream. Tellus, 3, 1-14.

Gent, P.R., and J.C. McWilliams, 1990: Isopycnal mixing in ocean circulation models. J. Phys. Oceanogr., 20, 150-155.

Gill, A.E., 1982: Atmosphere-ocean dynamics, Academic Press, San Diego, 662 pp. 
Gill, A.E., J.S.A. Green, and A. Simmons, 1974: Energy partition in the large-scale ocean circulation and the production of mid-ocean eddies. Deep-Sea Res., 21. 499-528.

Gille, S.T., M.M. Yale, and D.T. Sandwell, 2000: Global correlation of mesoscale ocean variability with seafloor roughness from satellite altimetry. Geophys. Res. Lett., 27, 1251-1254.

Green, J., 1970: Transfer properties of the large-scale eddies and the general circulation of the atmosphere. Quart. J. Roy. Meteor. Soc., 96, 157-185.

Haidvogel, D.B., and I.M. Held, 1980: Homogeneous quasigeostrophic turbulence driven by a uniform temperature gradient. J. Atmos. Sci., 37, 2644-2660.

Held, I.M., and V.D. Larichev, 1996: A scaling theory for horizontally homogeneous, baroclinically unstable flow on a beta plane. J. Atmos. Sci., 53, 946-952.

Hogg, N.G., 1985: Evidence for baroclinic instability in the Gulf Stream recirculation. Prog. Oceanogr., 14, 209-229.

Hogg, N.G., 1988: Stochastic wave radiation by the Gulf Stream. J. Phys. Oceanogr., 18, 1687-1701.

Hogg, N.G., 1993: Toward parameterization of the eddy field near the Gulf Stream. Deep-Sea Res., 40, 2359-2376.

Hogg, N.G., 1994: Observations of Gulf Sream meander-induced disturbances. Deep Sea Res., 24, 2534-2545.

Holopainen, E.O., 1961: On the effect of friction in baroclinic waves. Tellus, 13, 363-367.

Hua, B.L., and D.B. Haidvogel, 1986: Numerical simulations of the vertical structure of quasi-geostrophic turbulence. J. Atmos. Sci., 43, 2923-2936.

Kamenkovich, I.V., and J. Pedlosky, 1996: Radiating instability of nonzonal ocean currents. J. Phys. Oceanogr., 26, 622-643.

Keffer, T., 1985: The ventilation of the world's oceans: maps of the potential vorticity field. J. Phys. Oceanogr., 15, 509-523.

Kraichnan, R.H., 1967: Inertial ranges in two-dimensional turbulence. Phys. Flu$i d s, 10,1417-1423$.

Kundu, P.K., 1990: Fluid mechanics. Academic Press, San Diego, 638 pp. 
Kuo, A.C., and L.M. Polvani, 2000: Nonlinear geostrophic adjustment, cyclone/anticyclone asymmetry, and potential vorticity rearrangement. Submitted to Physics of Fluids.

LaCasce, J.H., 1996: Baroclinic vortices over a sloping bottom. PhD dissertation, Massachusetts Institute of Technology-Woods Hole Oceanographic Institution Joint Program.

Large, W.G., W.R. Holland, and J.C. Evans, 1991: Quasi-geostrophic ocean response to real wind forcing: the effects of temporal smoothing. J. Phys. Oceanogr., 21, 998-1017.

Larichev, V.D., and I.M. Held, 1995: Eddy amplitudes and fluxes in a homogeneous model of fully developed baroclinic instability. J. Phys. Oceanogr., 25, 22852297.

Larichev, V.D., and J.C. McWilliams, 1991: Weakly decaying turbulence in an equivalent-barotropic fluid. Phys. Fluids A, 3, 938-950.

Larichev, V.D., and G.M. Reznik, 1976: Two-dimensional Rossby soliton: an exact solution. Rep. U.S.S.R. Acad. Sci., 231, (5). Also POLYMODE News, 19.

Lee, S., and I.M. Held, 1991: Subcritical instability and hysteresis in a two-layer model. J. Atmos. Sci., 48, 1071-1077.

Lee, T.D., 1951: Difference between turbulence in a two-dimensional fluid and in a three-dimensional fluid. J. Appl. Phy., 22, 524.

Leith, C.E., 1968: Diffusion approximation for two-dimensional turbulence. Phys. Fluids, 11, 671-673.

Levitus, S., R. Burgett, and T. Boyer, 1994: World Ocean Atlas 1994. Vol. 3: Salinity; Vol. 4: Temperature. NOAA Atlas NESDIS 3 and 4, U.S. Dept. of Commerce.

Malanotte-Rizzoli, P., N.G. Hogg, and R.E. Young, 1994: Stochastic wave radiation by the Gulf Stream: Numerical experiments. Deep-Sea Res., 42, 389-423.

Maltrud, M.E., and G.K. Vallis, 1991: Energy spectra and coherent structures in forced two-dimensional and geostrophic turbulence. J. Fluid Mech., 228, 321-342.

McWilliams, J.C. 1984: The emergence of isolated coherent vortices in turbulent flow. J. Fluid Mech., 146, 21-43. 
McWilliams, J.C., 1985: Submesoscale coherent vortices in the ocean. Rev. Geophys., 23, 165-182.

McWilliams, J.C., and G.R. Flierl, 1979: On the evolution of isolated, nonlinear vortices. J. Phys. Oceanogr., 9, 1155-1182.

Mercier, H., and A. Colin deVerdiere, 1985: Space and time scales of mesoscale motions in the eastern North Atlantic. J. Phys. Oceanogr., 15, 171-183.

Muller, P., and C. Frankignoul, 1981: Direct atmospheric forcing of geostrophic eddies. J. Phys. Oceanogr., 11, 287-308.

Muller, T.J., and G. Siedler, 1992: Multi-year current time series in the eastern North Atlantic. J. Marine Res., 50, 63-98.

O'Dwyer, J.O., and R.G. Williams, 1997: The climatological distribution of potential vorticity over the abyssal ocean. J. Phys. Oceanogr., 27, 2488-2506.

Onsager, L., 1949: Statistical hydrodynamics. Nuovo Cimento, 6, Suppl. 6, 279. 287.

Owens, W.B., and F.P. Bretherton, 1978: A numerical study of mid-ocean mesoscale eddies. Deep-Sea Res., 25, 1-14.

Panetta, R.L., 1993: Zonal jets in wide baroclinically unstable regions: persistence and scale selection. J. Atmos. Sci., 50, 2073-2106.

Pedlosky, J., 1970: Finite amplitude baroclinic waves. J. Atm. Sci., 27, 15-30.

Pedlosky, J., 1975a: A note on the amplitude of baroclinic waves in the mid-ocean. Deep-Sea Res., 22, 575-576.

Pedlosky, J., 1975b: On secondary instability and the meridional scale of motion in the ocean. J. Phys. Oceanogr., 5, 603-607.

Pedlosky, J., 1975c: The amplitude of baroclinic wave triads and mesoscale motion in the ocean. J. Phys. Oceanogr., 5, 608-614.

Pedlosky, J., 1987: Geophysical fluid dynamics, Springer-Verlag, New York, second edition, $710 \mathrm{pp}$.

Peixoto, J.P., and A.H. Oort, 1992: Physics of climate, American Institute of Physics Press, New York, 520 pp.

Plumb, R.A., 1986: Three-dimensional propagation of transient quasi-geostrophic eddies and its relationship with the eddy forcing of the time-mean flow. $J$. Atmos. Sci., 43, 1657-1678. 
Polvani, L.M., J.C. McWilliams, M.A. Spall, and R. Ford, 1994: The coherent structures of shallow-water turbulence: deformation radius effects, cyclone/anticyclone asymmetry and gravity-wave generation. Chaos, 4, 177-186.

Polzin, K.L., J.M. Toole, J.R. Ledwell, and R.W. Schmitt, 1997: Spatial variability of turbulent mixing in the abyssal ocean. Science, 276, 93-96.

Rhines, P.B., 1975: Waves and turbulence on a beta plane. J. Fluid Mech., 69, 417-443.

Rhines, P.B., 1977: The dynamics of unsteady currents. The Sea, Vol. 6, E. Goldberg, Ed., John Wiley and Sons, New York, 189-318.

Richardson, P.L., 1983: Gulf Stream rings. Eddies in Marine Science, A.R. Robinson, Ed., Springer, Berlin, 19-45.

Richman, J.G., 1976: Kinematics and energetics of the mesoscale mid-ocean circulation: MODE. PhD dissertation, Massachusetts Institute of TechnologyWoods Hole Oceanographic Institution Joint Program.

Robinson, A.G., and J.C. McWilliams, 1974: The baroclinic instability of the open ocean. J. Phys. Oceanogr., 4, 281-294.

Salmon, R., 1978: Two-layer quasi-geostrophic turbulence in a simple special case. Geophys. Astrophys. Fluid Dyn., 10, 25-52.

Salmon, R., 1980: Baroclinic instability and geostrophic turbulence. Geophys. Astrophys. Fluid Dyn., 15, 167-211.

Salmon, R., 1998: Lectures on geophysical fluid dynamics, Oxford University Press, Oxford, $378 \mathrm{pp}$.

Schmitz, W.J., 1996: On the world ocean circulation. Vol. I: Some global features/North Atlantic circulation. Vol. II: The Pacific and Indian oceans/A global update. Woods Hole Oceanographic Institution Technical Reports, WHOI96-03 and WHOI-96-08.

Smith, K. S. and G. K. Vallis, 2000: The scales and equilibration of mesoscale ocean eddies. Part I: freely evolving flow. (submitted to J. Phys. Oceanogr.).

Spall, M.A., 1994: Mechanism for low-frequency variability and salt flux in the Mediterranean salt tongue. J. Geophys. Res., 99, 10121-10129.

Spall, M.A., 2000: Generation of strong mesoscale eddies by weak ocean gyres. $J$. Marine Res., 58, 97-116. 